\title{
Modeling and Simulation of Components in an Integrated Gasification Combined Cycle Plant for Developing Sensor Networks to Detect Faults
}

Pratik Pednekar

Follow this and additional works at: https://researchrepository.wvu.edu/etd

\section{Recommended Citation}

Pednekar, Pratik, "Modeling and Simulation of Components in an Integrated Gasification Combined Cycle Plant for Developing Sensor Networks to Detect Faults" (2016). Graduate Theses, Dissertations, and Problem Reports. 6393.

https://researchrepository.wvu.edu/etd/6393

This Dissertation is protected by copyright and/or related rights. It has been brought to you by the The Research Repository @WVU with permission from the rights-holder(s). You are free to use this Dissertation in any way that is permitted by the copyright and related rights legislation that applies to your use. For other uses you must obtain permission from the rights-holder(s) directly, unless additional rights are indicated by a Creative Commons license in the record and/ or on the work itself. This Dissertation has been accepted for inclusion in WVU Graduate Theses, Dissertations, and Problem Reports collection by an authorized administrator of The Research Repository @ WVU.

For more information, please contact researchrepository@mail.wvu.edu. 


\title{
Modeling and Simulation of Components in an Integrated Gasification Combined Cycle Plant for Developing Sensor Networks to Detect Faults
}

\author{
Pratik Pednekar \\ Dissertation submitted to the \\ Benjamin M. Statler College of Engineering and Mineral Resources \\ at West Virginia University \\ in partial fulfillment of the requirements for the degree of \\ Doctor of Philosophy in \\ Chemical Engineering
}

Debangsu Bhattacharyya, Ph.D., Chair

Dr. Richard Turton, Ph.D.

Dr. Charter D. Stinespring, Ph.D.

Dr. Stephen E. Zitney, Ph.D.

Dr. Edward Sabolsky, Ph.D.

Department of Chemical Engineering

Morgantown, West Virginia

2016

Keywords: IGCC, sensor placement, fault simulation, modeling, gasifier, slag, refractory degradation sour water gas shift reactor

Copyright 2016 Pratik Pednekar 


\title{
ABSTRACT \\ Modeling and Simulation of Components in an Integrated Gasification Combined Cycle Plant for Developing Sensor Networks to Detect Faults
}

\author{
Pratik Pednekar
}

The goal of this work is to help synthesize a sensor network to detect and diagnose faults and to monitor conditions of the key equipment items. Faults or events that lead to loss in productivity occur over time. These faults, if not detected and mitigated at an early stage, can lead to severe loss in productivity, efficiency, and equipment damage, and can be a safety hazard. The desired algorithm for sensor network design would provide information about the number, type and location of sensors that should be deployed for fault diagnosis and condition monitoring of a plant. In this work, the focus was on the integrated gasification combined cycle (IGCC) power plant where the faults at the equipment level and the plant level are considered separately. At the plant level, the objective is to observe whether a fault has occurred or not and identify the specific fault. For component-level faults, the objective is to obtain quantitative information about the extent of a particular fault. For the model-based sensor network design, high-fidelity process model of the IGCC plant is the key requirement.

For component level sensor placement, high-fidelity partial differential algebraic equation (PDAE)-based models are developed. Mechanistic models for faults are developed and included in the PDAE-based models. For system-level sensor placement, faults are simulated in the IGCC plant and the dynamic response of the process is captured. Both the steady-state and dynamic information are used to generate markers that are then utilized for sensor network design.

Whether faults in a particular equipment item should be considered at the unit level or system level depend on the criticality of the equipment item, its likelihood to failure, and the resolution desired for specific faults. In this work, the sour water gas shift reactor (SWGSR) and the gasifier are considered at the unit level. Fly ash may get deposited on the SWGSR catalyst and in the voids in the SWGSR resulting in decreased conversion of carbon monoxide. A MATLABbased PDAE model of the SWGSR has been developed that considers key faults such as changes in the porosity, surface area, and catalyst activity. In a slagging gasifier, the molten slag that flows along the inner wall can penetrate into the refractory layer, and due to chemical corrosion and thermal and mechanical stress eventually result in thinning or spalling of the refractory. Extent of penetration of slag into the refractory wall and the spalling of the refractory are considered to be important variables for condition monitoring of the gasifier. In addition, as an increasing slag layer thickness can eventually lead to shutdown of the gasifier yet the slag layer thickness cannot be directly measured using the current measurement technology, slag layer thickness is also considered to be an important variable for condition monitoring. For capturing the slag formation, and detachment phenomena accurately, a novel hybrid shrinking coreshrinking particle (HSCSP) model is developed. For tracking the detached slag droplets and the char particles along the gasifier, a particle model is developed and integrated with the HSCSP model. A slag model is developed that captures the process of the detachment of the slag droplets from the char surface, transport of the droplets towards the wall, deposition of a fraction of the droplets on the wall and formation of a slag layer on the wall. Finally, a refractory degradation 
model is developed for calculating the penetration of the slag inside the wall and the size and time for a spall to occur due to the combined effects of volume change as a result of slag penetration as well as thermal and mechanical stresses.

System-level models are enhanced and faults are simulated spanning across various sections of the IGCC plant. For example, in the SELEXOL-based acid gas removal unit the available area in the trays of distillation columns may get reduced due to deposition of solids. This can result in loss of efficiency. Leakages in heat exchangers in this unit can result in the loss of expensive solvent or hazardous gases. In the combined cycle section, faults such as leakages and fouling in the heat exchangers, increased loss of heat through the combustor insulation that can result in loss of efficiency are simulated.

Sensor placement using a "two-tier" approach is also performed by developing a sensor network for a combined system that includes unit level as well as system level faults. A model of the gasification island is developed by integrating the SWGSR model developed in MATLAB with the model of the rest of the plant developed in Aspen Plus Dynamics. Since the two models are developed using different software platforms, an integration framework is developed that couples and synchronizes the two dynamic models. The sensor network obtained using the models developed in this work is found to be effective in observing and resolving faults both at the unit level as well as the plant level. 


\section{$\underline{\text { Dedication }}$}

I would like to dedicate this work to my parents, Chandrakant and Smita Pednekar for all their love and support through this and all other journeys in my life; my brother, Sidhant Pednekar, for keeping my spirits up and being there for me and to Ruchi Thakkar, for her encouragement and dedication. 


\section{$\underline{\text { Acknowledgement }}$}

I would like to thank my advisor, Dr. Debangsu Bhattacharyya, for being a great mentor through this project. I am grateful for his guidance, patience and support and for always making time to discuss and work through some new and interesting problems through this journey.

I am also very grateful to my advisors Dr. Richard Turton and Dr. Raghunathan Rengaswamy. I appreciate Dr. Turton for his keen insight and guidance in the project. I thank Dr. Rengaswamy for his encouragement and zest during my $\mathrm{PhD}$ and for all the discussions we have had over our conference calls.

I would like to thank my committee members, Dr. Stephen Zitney, Dr. Edward Sabolsky and Dr. Charter Stinespring for their support, advice and direction in this research work.

I am thankful to Parham Mobed and Jeevan Maddala for being great partners in this project. Thanks to all my fellow lab members, Dustin, Job, Jacob, Prokash, Qiang, Yuan, Qiao, Anderson, Josh, Ben, Samson, Srinivas, Danny and Esha for their great company and for keeping things fun and interesting in the lab.

I'd like to thank Saurabh, Sanket, Akshaya, Rutika, Srinath, Madhur, Tanya, Deepa, Manohar, Nagashree, Siddhita, Tarannum, Reem and many others for making my stay at Morgantown exciting and memorable. Thanks also to my dear friends Kiran, Chetan, Kartikeya and Ashish for being there for me.

I would like to thank National Energy Technology Laboratory (NETL) of U.S. Department of Energy (DOE) for providing the funding to this project. 


\section{$\underline{\text { List of Figures }}$}

Figure 1.1: Flowchart for sensor placement at system-level

Figure 1.2: Algorithm used to synthesize a sensor network for condition monitoring at the component level

Figure 1.3: System Block Diagram of the IGCC plant

Figure 2.1: General plot showing the relation between the $\mathrm{V}_{\text {dep }}$ and $\tau_{+}$redrawn

Figure 3.1: Hybrid shrinking core shrinking particle (HSCSP) model

Figure 3.2: Slag formation and detachment

Figure 3.3: Schematic of solid phase consisting separated slag, attached slag, and char particle.

Figure 3.4: Schematic of the recirculation model

Figure 3.5: Continuum phase domain for solid and gas integrated with the particle phase domain.

Figure 3.6: Schematic for the slag droplet formation and detachment model

Figure 3.7: Schematic of information exchange between the continuum model and particle model Figure 3.8: Comparisons of the mole fractions of $\mathrm{CO}_{2}, \mathrm{CO}, \mathrm{H}_{2}$ and $\mathrm{H}_{2} \mathrm{O}$ (on dry basis) at the exit of the RSC with TECO data

Figure 3.9: Comparison of the reaction rates between the shrinking particle model and hybrid shrinking core-shrinking particle model

Figure 3.10: Comparison of carbon conversion of carbon between the gasifier model and the complete coalescence model

Figure 3.11: Comparison of solids temperature profiles between the shrinking core and HSCSP model

Figure 3.12: Variation of diameter of char particles, attached slag droplets, and average density of the char-slag system along the gasifier

Figure 3.13: Variation of $\varepsilon_{\mathrm{sa}}$ along the gasifier for complete coalescence case

Figure 3.14: Variation of $\varepsilon_{\mathrm{sa}}$ along the gasifier for the slag detachment scenario

Figure 3.15: Variation of detached slag volume fraction along the gasifier

Figure 3.16: Variation of $\mathrm{p}_{\mathrm{sl}}$ along the gasifier for 10 and 15 micron case

Figure 4.1: Schematic of the formation mechanism of slag droplets and their deposition on the

wall along with char particles and the subsequent formation of a slag layer on the gasifier wall

Figure 4.2: Schematic representation of the notations for denoting solids fractions in the continuum model

Figure 4.3: Transfer of information between the particle model and continuum model

Figure 4.4: Schematic showing the slag droplet deposition on the gasifier wall

Figure 4.5: Schematic of the mass, momentum and energy interactions in the slag layer

Figure 4.6: Control structure implemented to simulate dynamic runs for the slagging gasifier

Figure 4.7: Deposition flux and slag layer thickness profile for base case PSD

Figure 4.8: Slag droplet deposition flux and slag layer thickness for cases SD-5-50 and SD 60-10

Figure 4.9: Comparison of the slag layer thickness for Case CC and base case

Figure 4.10: Effect of change in $\mathrm{O}_{2} /$ coal ratio on slag layer thickness at gasifier exit

Figure 4.11: Variation of the maximum and minimum slag layer temperature due to change in $\mathrm{O}_{2} /$ Coal feed ratio

Figure 4.12: Effect of silica ratio on exit viscosity and slag layer thickness

Figure 4.13: Effect of ramp increase in coal slurry flow rate on slag layer thickness at final CV

Figure 4.14: Effect of ramp increase in coal slurry flow rate on slag layer temperature at final CV 
Figure 4.15: Change in outlet gas composition and carbon conversion during coal switch from Illinois \#6 to Pittsburgh \#8 coal

Figure 4.16: Deposition flux before and after change of coal from Illinois \#6 to Pittsburgh \#8 Figure 4.17: Slag layer temperature and viscosity profile before and after the coal switch.

Figure 4.18: Transient response of slag layer and wall temperature and slag thickness at gasifier exit

Figure 5.1: Temperature and diffusivity profile across the high chromia layer.

Figure 5.2: Boundary and interface conditions for stress in the multi-layer refractory wall

Figure 5.3: Slag penetration into the high chrome refractory at different time instances

Figure 5.4: Steady state temperature profile along the refractory across the four layers

Figure 5.5: Coal slurry set point variation in the gasifier model

Figure 5.6: Wall temperature at the grid point selected for simulating degradation

Figure 5.7: Time for first spall for the four cases

Figure 5.8: Radial stress profile across the high chrome, alumina and silica layers

Figure 5.9: Hoop stress profile across the high chrome, alumina and silica layers

Figure 5.10: Effect of increase in temperature on the radial stress profile in the high chrome refractory

Figure 5.11: Effect of increase in temperature on the hoop stress profile in the high chrome refractory

Figure 5.12: Slag penetration profile in the high chrome refractory layer with time

Figure 5.13: Temporal change in the radial stress profile of the high chrome refractory

Figure 5.14: Temporal change in the hoop stress profile of the high chrome refractory

Figure 6.1: Schematic of the single stage SWGSR model

Figure 6.2: CO conversion profiles for different inlet gas temperatures_Figure 6.3: COS

conversion profiles for different inlet gas temperatures

Figure 6.3: COS conversion profiles for different inlet gas temperatures

Figure 6.4: $\mathrm{CO}$ conversion profile for different steam/CO molar ratio

Figure 6.5: Required (a) Diameter (b) Flow at different steam/CO molar ratios

Figure 6.6: CO conversion transient for a step change in inlet temperature from 620 to $640 \mathrm{~K}$

Figure 6.7: Temperature transient at the outlet of the reactor for a step change in inlet

temperature from 620 to $640 \mathrm{~K}$

Figure 6.8: Effect of catalyst deactivation over time on $\mathrm{CO}$ conversion over 5 years

Figure 6.9: Effect of catalyst deactivation over time on COS conversion over 5 years

Figure 6.10: Schematic showing the two-stage SWGSR system

Figure 6.11: Change in CO mole fraction at the exit of the SWGSR due to faults

Figure 6.12: Change in COS mole fraction at the exit of the SWGSR due to faults

Figure 6.13: Change in outlet temperature of the SWGSR due to faults.

Figure 6.14: Temperature profile along reactor R2

Figure 6.15: Profile of CO mole fraction along the length of reactor R2

Figure 6.16: Plot of COS mole fraction along R2

Figure 7.1: Schematic for SELEXOL unit

Figure 7.2: Schematic of the combined cycle power plant

Figure 7.3: Number of irresolvable faults (left) and the corresponding sensor network cost (right)

at different MR threshold values

Figure 7.4: Schematic representation of the Sour Water Gas Shift Reactor system developed in

MATLAB 
Figure 7.5: Exchange of information between the SWGSR in MATLAB and the SELEXOL model in APD

Figure 7.6: HE configuration 1: leak simulated as mixing of inlet high pressure stream into outlet low pressure stream

Figure 7.7: HE configuration 2: leak simulated as mixing of outlet high pressure stream into inlet low pressure stream

Figure 7.8: $\mathrm{CO}$ at the end of $\mathrm{R} 1$ as a result of a ramp decrease in the porosity

Figure 7.9: $\mathrm{COS}$ at the end of $\mathrm{R} 1$ as a result of a ramp decrease in porosity

Figure 7.10: Temperature at end of $\mathrm{R} 1$ as a result of a ramp decrease in porosity

Figure 7.11: $\mathrm{CO}$ at end of $\mathrm{R} 2$ as a result of ramp decrease in porosity

Fig. 7.12: COS at end of $\mathrm{R} 2$ as a result of ramp decrease in porosity

Figure 7.13: Temperature response at the exit of R2 as a result of ramp decrease in porosity

Figure 7.14: CO response at the exit of the Selexol unit as a result of ramp decrease in porosity Figure 7.15: GA progress for different faults

Figure 7.16: Fault severity estimation using optimal solution for fault (a) $F_{25}$ (b) $F_{26}$ (c) $F_{27}$ (d)

$F_{31}(\mathrm{e}) \mathrm{F}_{32}$

Figure A.1: Flowchart of FES algorithm in presence of numerical solution

Figure A.2: Magnitude ratio for example in Table A.3

Figure A.3: Flowchart of MR algorithm

Figure B.1: Flowchart showing the EKF algorithm 


\section{$\underline{\text { List of Tables }}$}

Table 2.1: Solid phase reactions

Table 3.1: Kinetic parameters of the heterogeneous reactions in the gasifier

Table 3.2: Kinetic parameters of the homogeneous reactions in the gasifier

Table 3.3: Model parameters and input conditions

Table 3.4: Proximate and Ultimate analysis of Illinois \#6 coal

Table 3.5: Validation data from TECO power plant

Table 3.6: Comparison between outlet mole fractions of SCM and HSCSP models

Table 4.1: Model parameters and input conditions

Table 4.2: Proximate and Ultimate analysis of Illinois \#6 and Pittsburgh \#8 coal [As-Received (wt \%)]

Table 4.3: Parameters for slag flow layer

Table 4.4: Comparison of the results from this work with the existing literature (Bockelie, 2002;

Monaghan, 2012)

Table 4.5: Simulated particle size distributions

Table 4.6: Silica ratios calculated for Illinois \#6 coal taken from literature

Table 5.1: Physical constants used in the stress model

Table 6.1: Simulation condition (Woods et al., 2007)

Table 6.2: Legend for Figures 6.3-6.5

Table 7.1: List of faults simulated in the SELEXOL unit

Table 7.2: List of measured variables in SELEXOL process

Table 7.3: Results of applying different algorithms to SELEXOL process

Table 7.4: Faults at different severity level simulated in the combined cycle model

Table 7.5: Weight and threshold of each variable in the sensor placement algorithms

Table 7.6: SDG and FES algorithms results

Table 7.7: MR algorithm results

Table 7.8: Faults simulated on the SELEXOL side of the integrated model

Table 7.9: Faults simulated on the SWGSR side of the integrated model

Table 7.10: System-level sensor placement results of gasification island

Table 7.11: List of sensors for FES \& MR algorithm with low MR threshold level

Table 7.12: Optimal location and type of sensors for different faults

Table 7.13: Comparison of normalized fitness values for GA solution and final sensor network

Table A.1: Thresholds for various sensors used in this work

Table A.2: Type and cost of each sensor used in all case studies

Table A.3: SDG example to compare with MR based approach

Table B.1: Time update equations

Table B.2: Measurement update equations

Table B.3: EKF parameters for the Water gas shift reactor in the gasification island model 


\section{Contents}

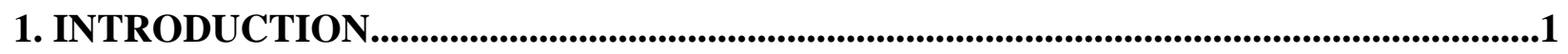

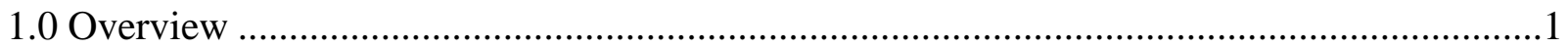

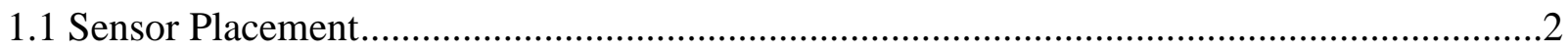

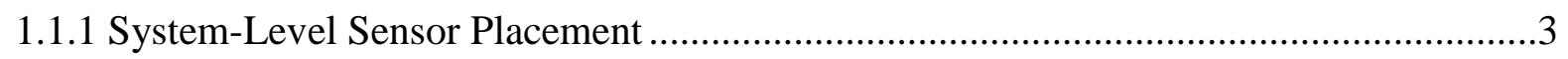

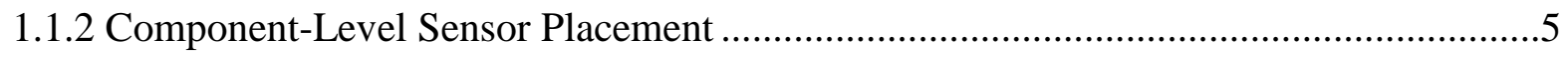

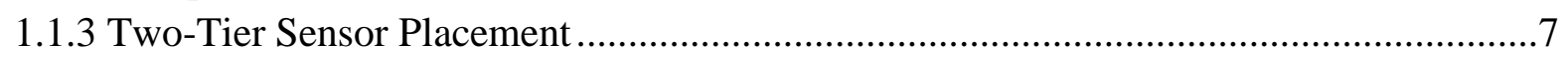

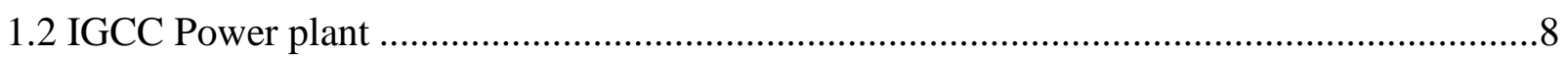

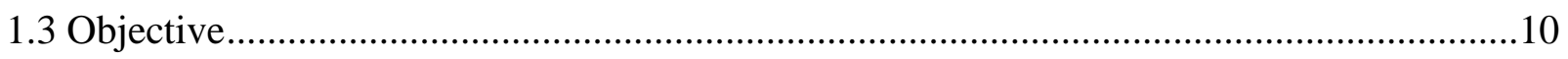

1.3.1 Specific Objectives.............................................................................................

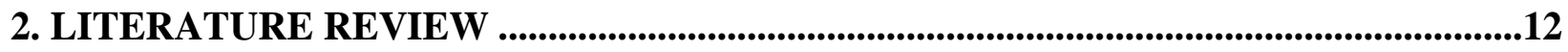

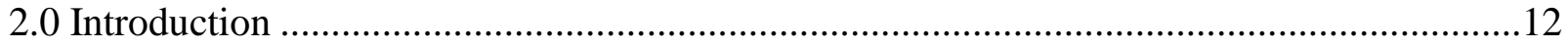

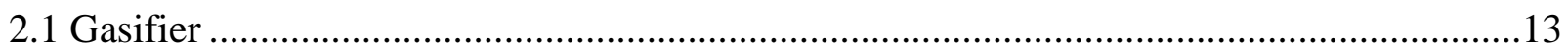

2.1.1 Reactions and Processes .........................................................................................13

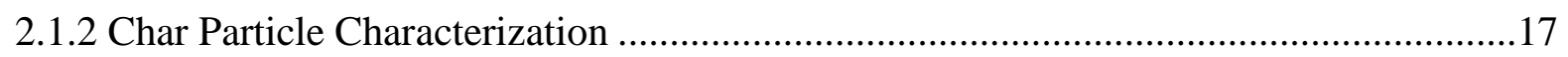

2.1.3 Slag Detachment and Droplet Sizes .......................................................................18

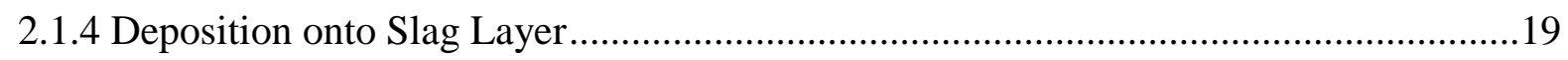

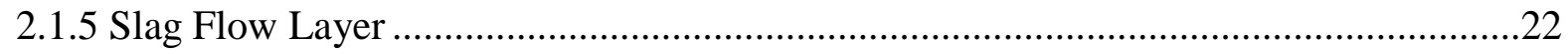

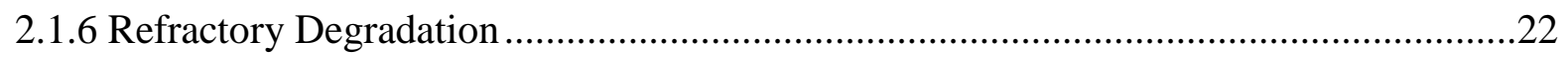

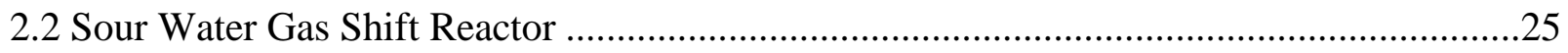

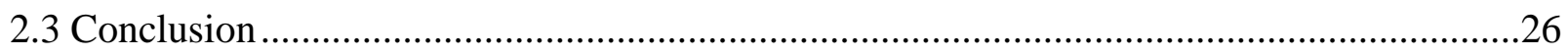

3. GASIFIER WITH SLAG MODELING ............................................................................28

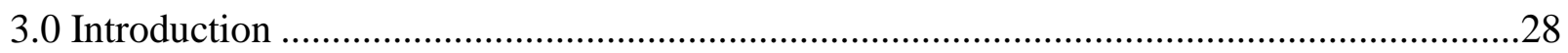

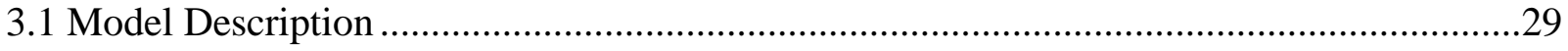

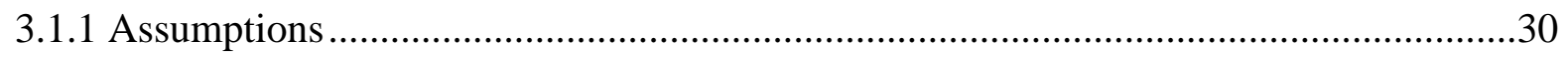

3.1.2 Hybrid Shrinking Core Shrinking Particle Model ….......................................................31

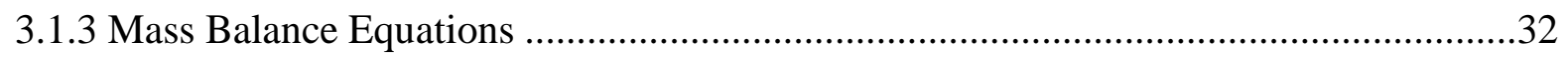

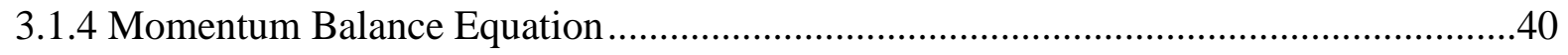

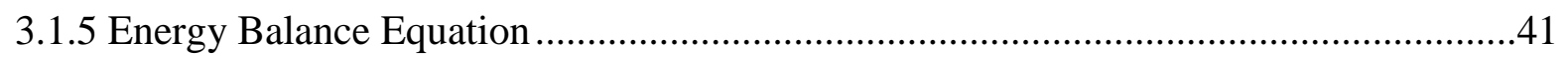

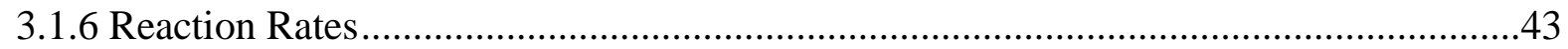

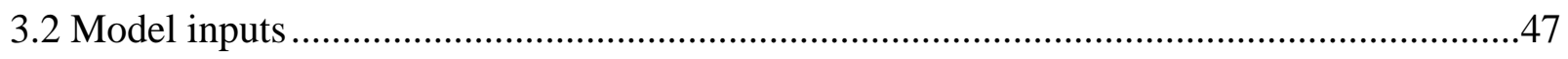

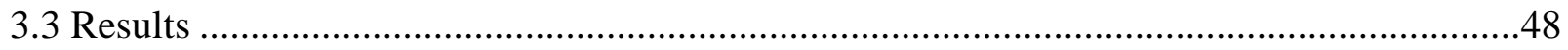

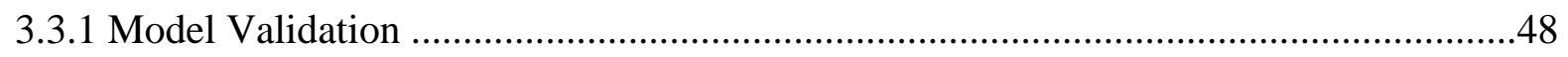

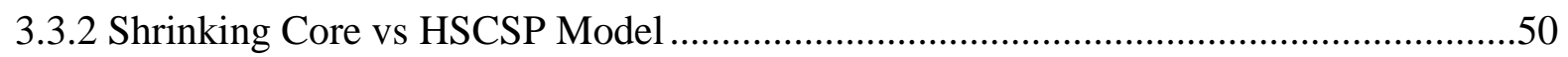

3.3.3 Complete Coalescence Case ……….......................................................................5

3.3.4 Slag Formation and Detachment Results ………..................................................5

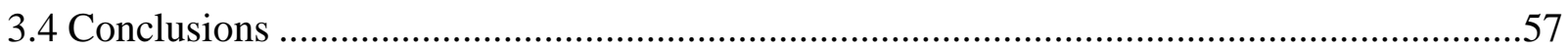

4. GASIFIER MODEL WITH SLAG DEPOSITION AND FLOW ........................................58

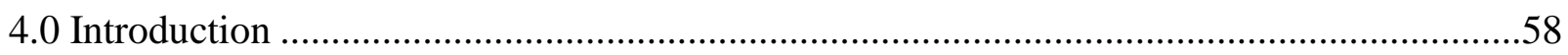




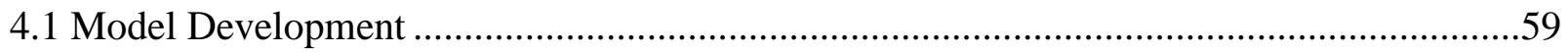

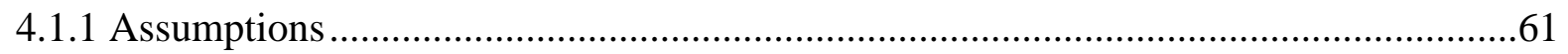

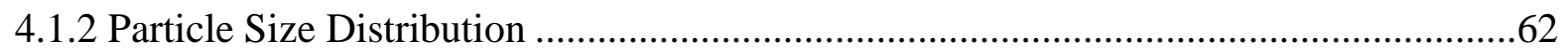

4.1.3 Incorporating PSD into Mass Balance Equations ………........................................62

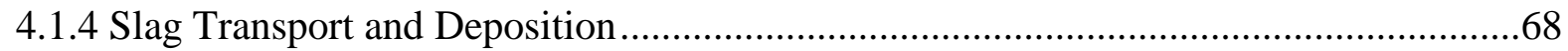

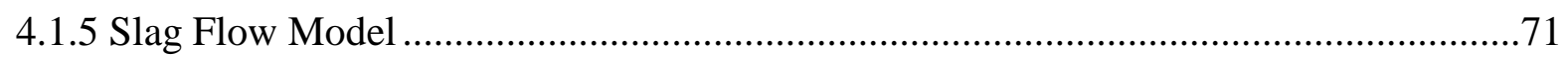

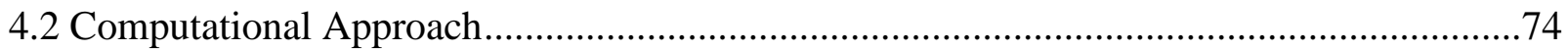

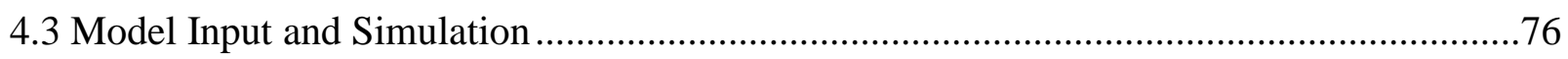

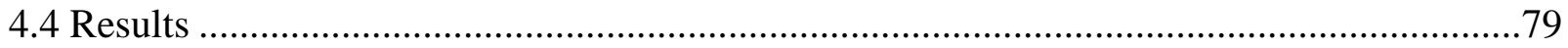

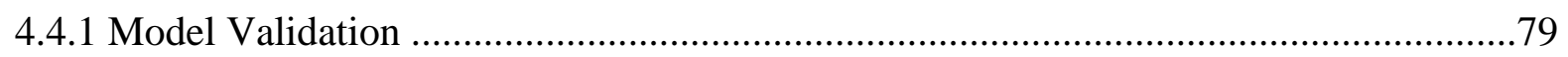

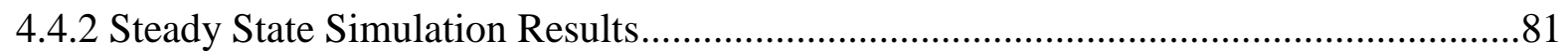

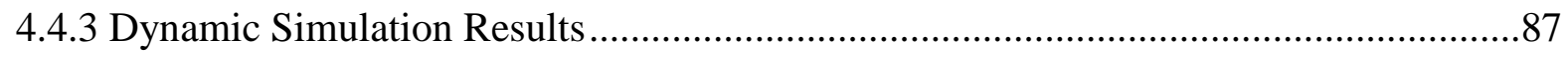

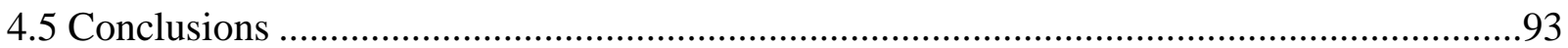

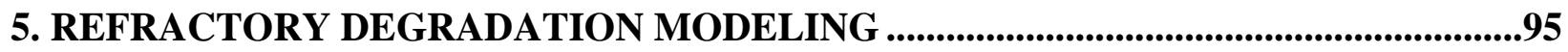

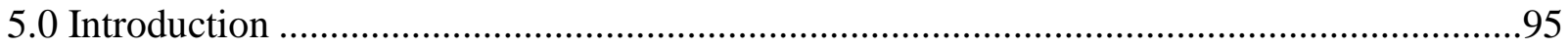

5.1 Refractory degradation - compressive spall modeling ………..........................................96

5.1.1 Refractory model with slag penetration ..................................................................96

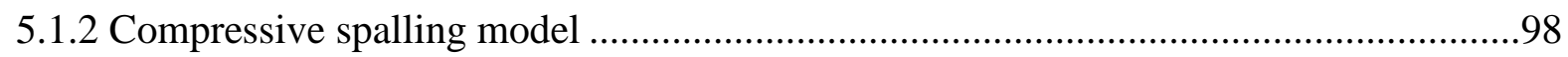

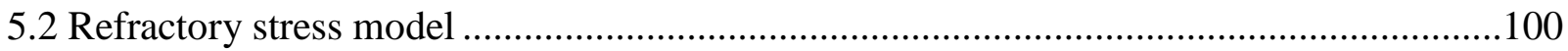

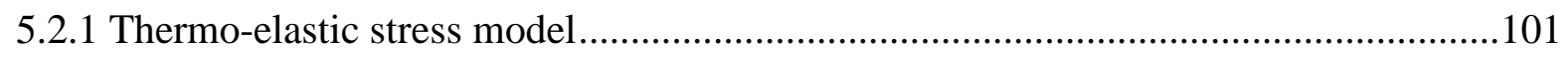

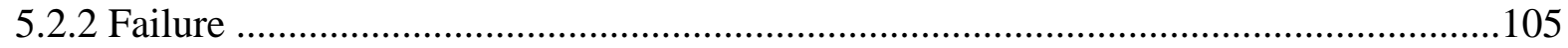

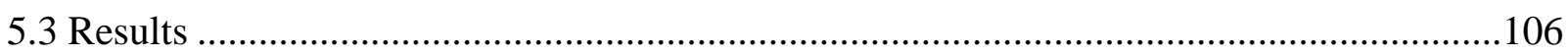

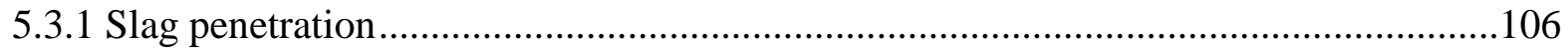

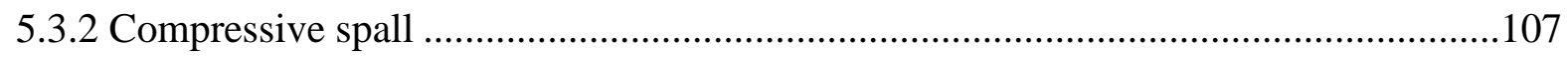

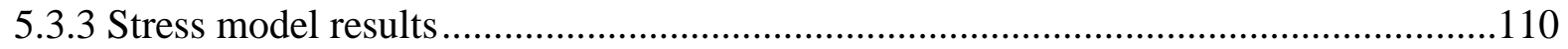

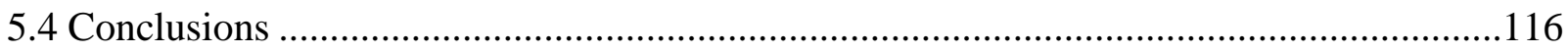

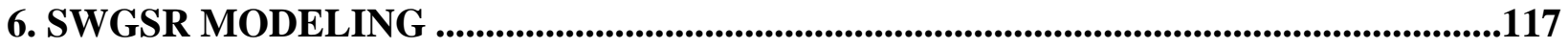

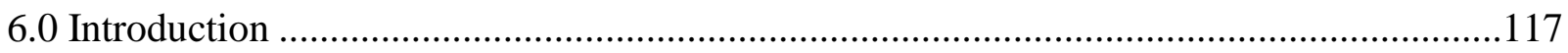

6.1 Model Development - Single Stage SWGSR ……….............................................117

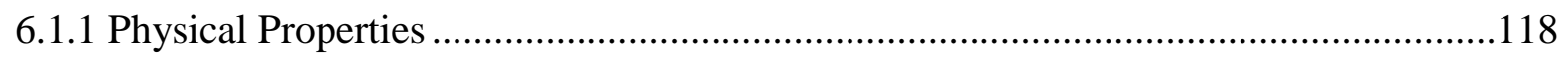

6.1.2 Model Equation for Catalyst Pellets .........................................................................120

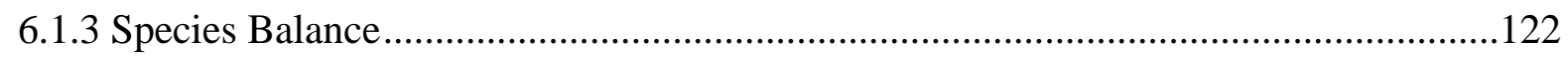

6.1.4 Momentum Balance ..........................................................................................122

6.1.5 Gas Phase Energy Balance ...............................................................................123

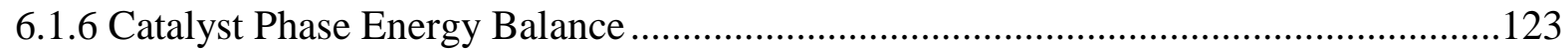

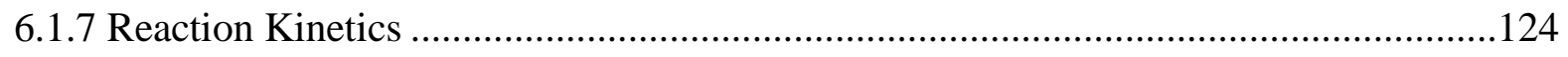

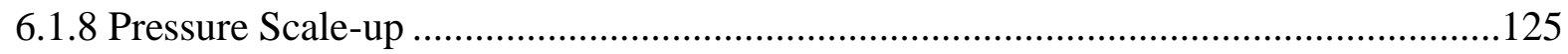

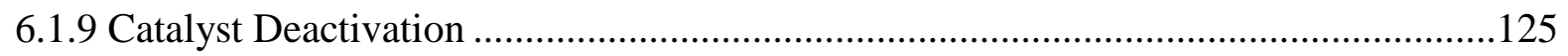

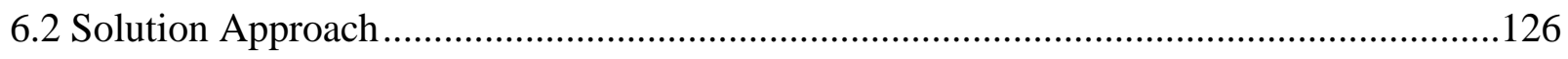

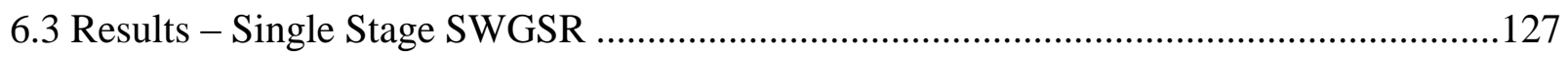




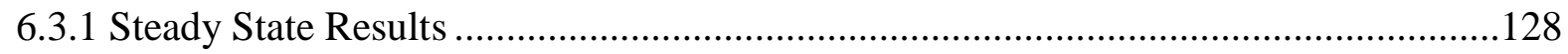

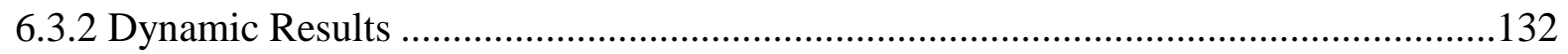

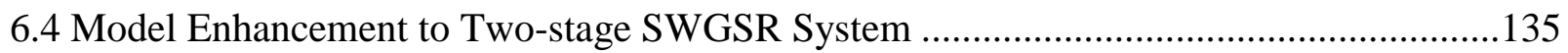

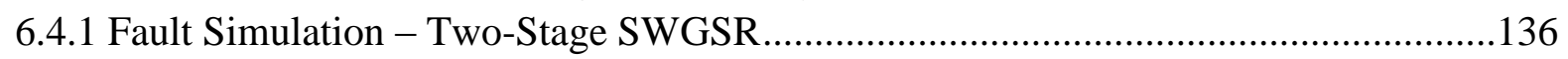

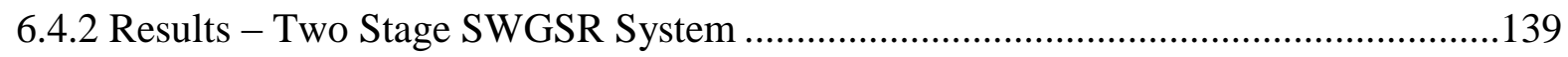

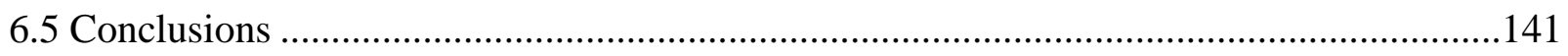

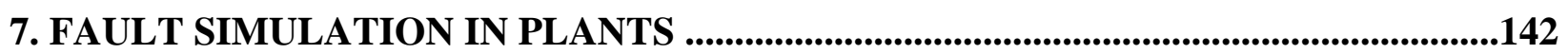

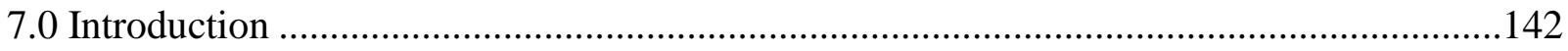

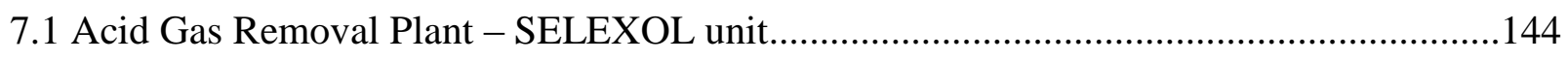

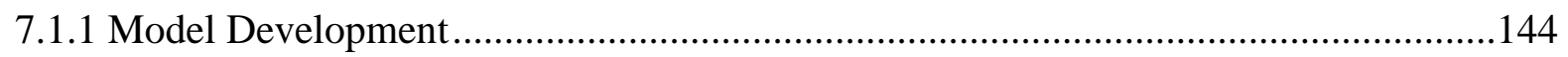

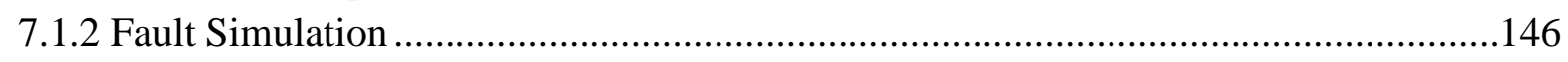

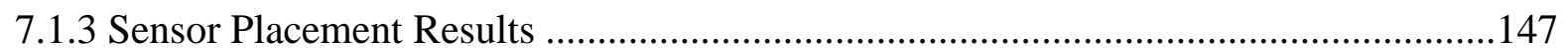

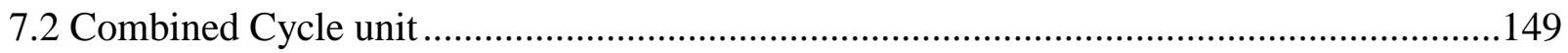

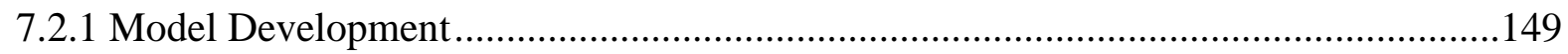

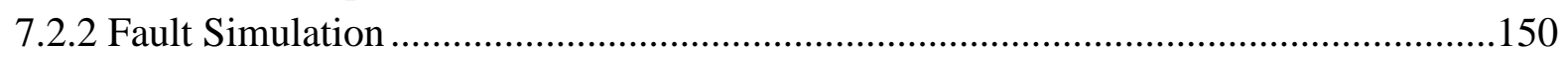

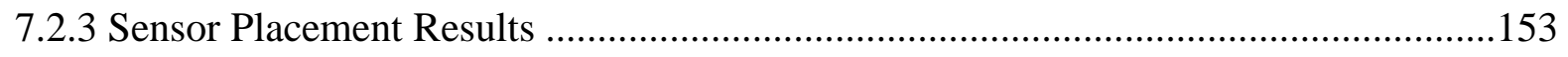

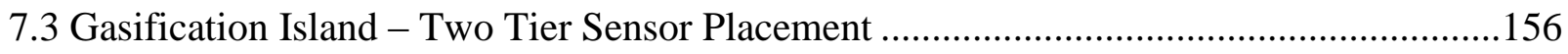

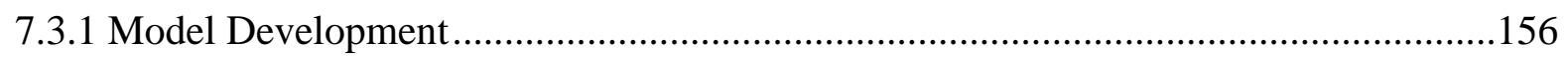

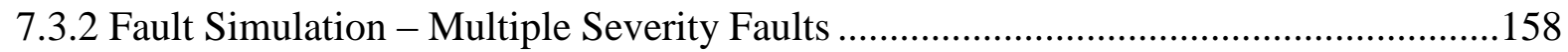

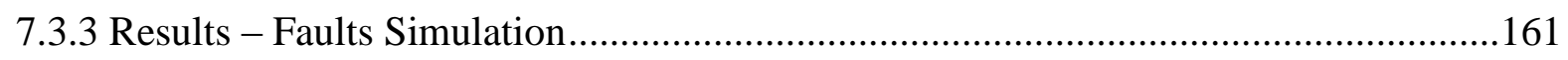

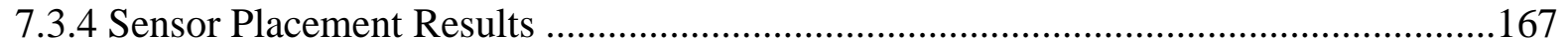

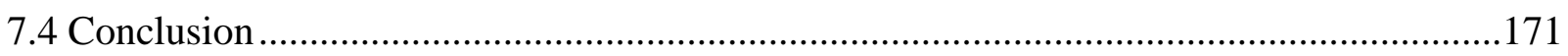

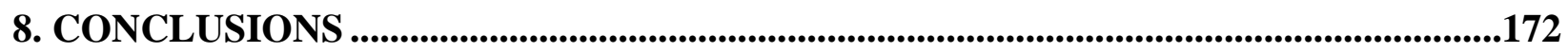

9. RECOMMENDATIONS AND FUTURE WORK .................................................................175

REFERENCES.............................................................................................................................178

APPENDIX A: SYSTEM-LEVEL SENSOR PLACEMENT ................................................189

APPENDIX B: COMPONENT-LEVEL SENSOR PLACEMENT .........................................198

APPENDIX C: DERIVATION FOR ANALYTICAL EQUATIONS FOR STRESS ..........204 


\section{INTRODUCTION}

\subsection{Overview}

Carbonaceous feeds for energy production are present in abundance in many regions in the US and it is estimated that these reserves could provide energy for several centuries to come. Presently, in the US, about $40 \%$ of the electricity is generated from coal. With stricter environmental regulations, the demand for clean power generation sources will become a key deciding factor in the use and development of coal based power generation technologies. Integrated gasification combined cycle (IGCC) technology could provide a means to obtain clean power and efficiently converting coal to energy. Plants with this technology benefit from the advantages of gasification that include high feedstock flexibility and high partial pressure of carbon dioxide $\left(\mathrm{CO}_{2}\right)$ resulting in efficient $\mathrm{CO}_{2}$ capture. The configuration of the IGCC makes it a highly competitive technology for producing energy efficiently and in an environment-friendly manner.

Early detection of faults in the IGCC process can be helpful in improving the availability and efficiency of these plants. For fault detection and diagnosis, the sensor network plays a key role. Some faults occur at locations where the placement of sensors is not possible. In addition, some faults cannot be measured directly with the current state of the art in the measurement technology. A sensor or a set of sensors can be placed at some other location so that its (their) response(s) can be used to monitor the equipment or diagnose a fault. The sensor cost should also be accounted for while placing the sensors. However, in a large-scale plant, there are trillions of possible combinations of sensors and therefore a systematic methodology is needed for designing the sensor network. A sensor placement study that provides the user with the ability to observe and resolve faults while using the minimum cost sensor network can be very useful. 


\subsection{Sensor Placement}

Sensors are of great importance in the chemical industry and are widely used for purposes of controls in plants. The use of sensors for the purposes of fault diagnosis can have several advantages. The early detection of faults can help mitigate the damage to the plant and reduced the overall downtime of the plant by accelerating the troubleshooting process. In addition, the plant would be better poised to meet the emission standards. Sensors that can monitor the extent of faults in equipment items can help better schedule for maintenance and develop operating procedures so as to maximize their life thereby increasing the efficiency of the plant. Information from the sensor network could also be useful to devise strategies to efficiently operate the plant in the presence of faults.

A model based strategy for sensor placement for fault detection and diagnosis has several unique advantages. It is infeasible to place sensors at every location in a plant due to hardware constraints as well as due to economic aspect and information overload. A model based strategy can identify a set of sensors that can detect the desired set of faults and provide reliable information in a cost effective manner. Sensors may not be able to provide information about faults directly, but may be used to infer them. Using model based sensor placement strategy, a diagnostic system can process the information available from the sensor network for detecting and diagnosing faults.

Sensor placement for fault detection and diagnosis would be particularly useful in equipment items that operate under extreme conditions. Hardware limitations or the limitations of measurement technology may make it difficult to measure a fault directly in such equipment items. For example, a gasifier operates under high temperature, pressure, and erosive environment. Even though the gasifier internal temperature is an important variable, the temperature sensors do not last long. In addition, it is infeasible to measure the extent of slag penetration or spall of the refractory using the current measurement technology. However, it may be feasible to utilize information obtained from sensors placed elsewhere to detect the faults for such processes.

The approach for model based sensor placement differs based on the level of information required for corrective action to be taken. Some faults may lead to plant shutdown or serious 
equipment damage or safety hazards and therefore, it is of immense interest to detect these faults immediately and take corrective action. For example, leakage in a heat exchanger can lead to mixing of chemicals resulting in a flammable mixture. For these faults, one is generally interested to know if the fault has occurred and therefore a qualitative model-based approach is often sufficient. Other faults can evolve slowly over period of time. For example, the catalyst in a reactor will start to deactivate as soon as the reactor starts operating. For such faults, magnitude of the faults as well as spatial location of the fault is of importance and therefore a quantitative model-based approach is desired.

In this work, the sensor placement is done at two levels viz. system-level and component-level. The objective is to develop an integrated two-tier approach to sensor placement and to study whether such an approach can provide additional advantages as opposed to sensor network algorithms that are developed at these levels separately and as a result, fail to take advantage of the synergistic information available from individual levels.

\subsubsection{System-Level Sensor Placement}

Highly integrated and complex processes and advancements in control technologies have increased the necessity of cost-effective automated diagnostic systems that can efficiently detect, identify and diagnose abnormalities and their origins as they propagate in the process. The diagnostic systems traditionally use underlying mathematical models and on-line measurements from a sensor network. When a fault occurs in a system, it affects the process variables, causing them to deviate from normal operating values. The diagnostic system compares the response of the sensor readings in the presence of faults with respect to reference values in order to detect and diagnose the faults.

For system level sensor placement (SP), in order to obtain the sensor network that can be used by such a diagnostic system, a qualitative approach for sensor placement is used. Two key criteria in fault detection and diagnosis (FDD) are fault observability and fault resolution. Fault observability is the ability of the network to detect the identified faults for which the SP is developed. For example, if one variable responds to all the faults selected, the faults are 
observable by that single sensor. On the other hand, fault resolution is the ability to identify the exact fault that has occurred.

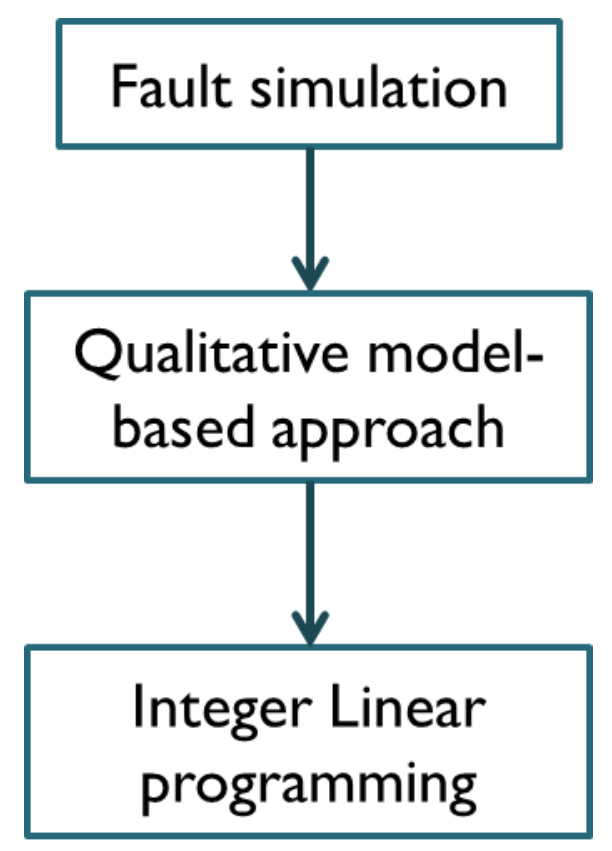

Figure 1.1: Flowchart for sensor placement at system-level

The flowchart for sensor placement at the system-level is shown in Figure 1.1. First, a process model of the system is required. The faults simulated in this work are selected based on the open literature and heuristics. The criteria for selecting the candidate measurement variables are the feasibility of placing a sensor in a particular location and existence of a commercial sensor for measuring a particular variable. The candidate sensors include flow, temperature, pressure, composition, and level sensors. Once the faults are simulated and the variables are recorded, a SP algorithm is used to select an optimal set of sensors for observing/resolving the faults.

There are several types of qualitative approaches that are used in the literature for fault detection and diagnosis. Approaches based on cause and effect models such as the directed graph (DG) and signed directed graph (SDG) are widely used for such purposes. The DG- or SDG-based approaches for SP take into account the response of a variable that exceeds a certain threshold due to a fault. For a DG-based approach, if the sensor response exceeds the threshold, it is assigned a value of ' 1 '; otherwise a value of ' 0 ' is assigned. The SDG also considers direction of change beyond the threshold to assign values of ' +1 ' or ' -1 ' to the candidate sensors. The 
constraint imposed for observability of all faults is that at least one sensor should be able to observe the fault. For resolving all the faults, the constraint is one or combination of sensors that can resolve each fault from all other faults. The sensor placement problem is posed as a binary integer linear programming (BILP) formulation by using the bipartite matrix while ensuring observability and maximum possible resolution.

In this work, the DG and SDG approaches are used under the assumption that a numerical solution is available. A new magnitude ratio (MR) based approach - ratio of the changes in a pair of process variables in response to a fault - is also used in the case of multiple process variables and multiple faults to improve sensor placements for FD. A fault evolution sequence (FES) approach - sequence in which a pair of sensor variables deviate from their nominal values in response to a fault - is also used for improvements in the sensor placements for FD. Details of these works and algorithms can be found in APPENDIX A.

\subsubsection{Component-Level Sensor Placement}

A diagnostic system that can monitor the condition of equipment in real time can be very useful in developing operational strategies, improving the equipment life, planning in advance for maintenance and avoiding unsafe operating conditions. It can be difficult to measure faults in equipment items directly. For example, no sensor can measure the change in porosity or deactivation of a catalyst, or the thinning of refractory in combustors and gasifiers. A modelbased estimator with optimal measurements can be very useful for faults in these systems.

An algorithm to obtain the sensor network for condition monitoring of an equipment item is shown in Figure 1.2. A high fidelity model of the equipment item that includes fault sub-models is developed and used in the framework of an extended Kalman filter (EKF) algorithm to estimate states in the partial differential algebraic equation (PDAE) based equipment model. White Gaussian distribution of noise is considered in the measurements and the process model. The EKF algorithm is explained in APPENDIX B. 


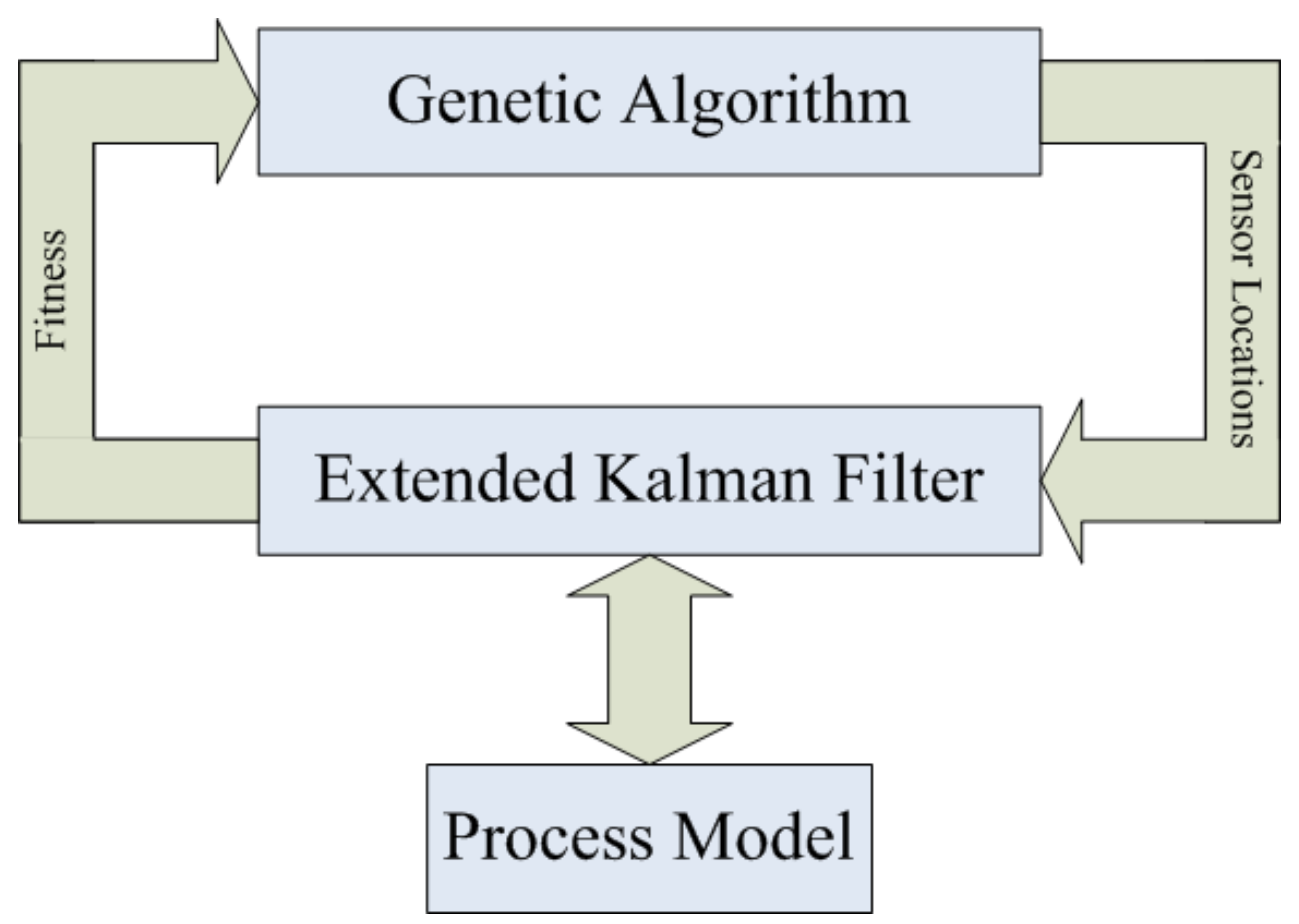

Figure 1.2: Algorithm used to synthesize a sensor network for condition monitoring at the component level

Faults are included as states and estimated by the extended Kalman filter (EKF) along with other states in the system. The genetic algorithm (GA) is used at an upper level that searches for an optimal sensor network. The genes are assumed to be measurement models and each measurement model represents a vector of binary decision variables in which if a sensor is placed, the decision variable will take a value of " 1 " else a value of "0" is assigned. The fitness function is calculated based on the estimates of the desired variables or time-varying parameters and an integer programming (IP) problem is solved until the desired tolerance for the estimates is obtained. 


\subsubsection{Two-Tier Sensor Placement}

A two-tier sensor placement strategy utilizes the sensors at the component level as well as the system level. It may be possible that some faults can be detected more easily in downstream equipment, where the effect of the fault might get magnified. An example from the IGCC unit is that deactivation in the sour water gas shift reactor catalyst could result in less amount of $\mathrm{H}_{2} \mathrm{~S}$ being produced through the COS hydrolysis reaction assuming that the syngas contains considerable amount of COS. Since the mole fraction of $\mathrm{H}_{2} \mathrm{~S}$ is very small, a composition sensor placed at the exit of the reactor may not detect this change. However, the flow rate of $\mathrm{H}_{2} \mathrm{~S}$ captured in the acid gas removal (AGR) unit will decrease and this change could be detected more easily. A flow sensor could be used instead of a composition sensor. Additional sensors placed downstream can also increase the estimation accuracy of the unmeasured variables.

On the other side, faults in the downstream units may also affect the equipment upstream due to the pressure-flow interaction. These faults can result in an increase in pressure at the outlet of the equipment item, thereby decreasing the flow or changing the pressure profile in the reactor and therefore can affect the reaction rates. Thus the synergistic effects of system-level and component-level faults can be exploited not only to obtain a cost-efficient sensor network, but also to obtain better observability and resolvability of faults. 


\subsection{IGCC Power plant}

Although, several configurations of IGCC plants are possible, the configuration considered in this work is similar to Case \#2 in the baseline DOE studies (Woods et al., 2007) that include a GEE-type oxygen-blown entrained-flow gasifier with $\mathrm{CO}_{2}$ capture and removal. Figure 1.3 shows a block diagram of an IGCC power plant. The key technologies in the IGCC process with $\mathrm{CO}_{2}$ capture are coal gasification, AGR, and combined cycle (CC) power generation technologies.

Coal is first processed to obtain the required particle size distribution and mixed with water before being fed to the gasifier as a slurry. High purity oxygen from the air separation unit (ASU) enters the gasifier. Syngas, which is a mixture consisting mainly of carbon monoxide (CO), hydrogen $\left(\mathrm{H}_{2}\right), \mathrm{CO}_{2}$ and water, exits the gasifier and is cooled in a radiant syngas cooler (RSC). Syngas is separated from slag and goes to a scrubber where it is scrubbed with water. Steam is then added to the syngas and it is sent to a two-stage sour water gas shift reactor (SWGSR) to produce $\mathrm{H}_{2}$ and $\mathrm{CO}_{2}$. The catalyst of the SWGSR is also able to convert the COS present in the syngas into $\mathrm{H}_{2} \mathrm{~S}$.

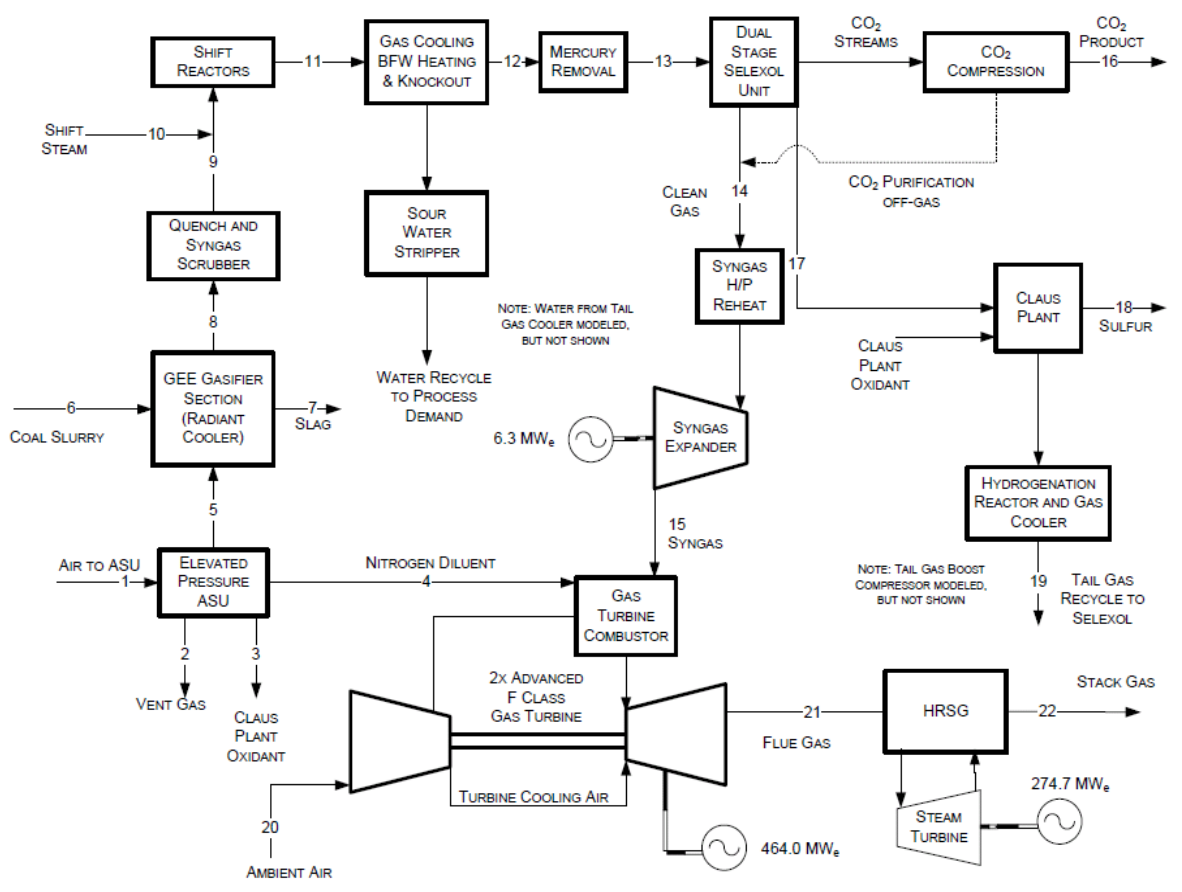

Figure 1.3: System Block Diagram of the IGCC plant 
The shifted syngas from the SWGSR is cooled in a series of heat exchangers and sent to the AGR unit. The AGR unit uses SELEXOL solvent to absorb $\mathrm{H}_{2} \mathrm{~S}$ and $\mathrm{CO}_{2}$ in a two-step physical absorption process. Captured $\mathrm{H}_{2} \mathrm{~S}$ is sent to the Claus-technology based sulfur recovery unit to produce elemental sulfur. Captured $\mathrm{CO}_{2}$ is processes, compressed, and sent for sequestration. The $\mathrm{H}_{2}$-rich syngas is reheated and sent to the gas turbine (GT). Nitrogen from the ASU is used as a diluent in the GT. Heat is recovered from the hot flue gas that leaves the GT by generating steam in a heat recovery steam generator (HRSG) that operates at three pressure levels. The steam is sent to a steam turbine (ST) producing more electricity. The flue gas from the HRSG is vented from the stack. 


\subsection{Objective}

The objective of the work is to develop/enhance process and equipment models of the IGCC plant and to identify and simulate faults using these models. These models are then used to identify sensor networks for fault diagnosis and component condition monitoring, respectively.

\subsubsection{Specific Objectives}

The following are the specific objectives of the work covered in the thesis

\subsubsection{Component Level}

\section{A. Gasifier}

i. To develop a high-fidelity PDAE-based model of a gasifier with a novel characterization of the char particles by accounting for the separation of slag droplets from the char particles.

ii. To develop a model for detachment of the slag droplets from the char surface, transport of the droplets towards the wall, deposition of a fraction of the droplets on the wall, and formation of a slag layer on the wall.

iii. To develop a refractory degradation model that can account for the temporal evolution of slag penetration into the refractory and can be used to calculate the size and time for a spall due to thermal and mechanical stresses

\section{B. Sour Water Gas Shift Reactor}

i. To develop a high-fidelity partial differential algebraic equation (PDAE)-based model of the SWGSR that can capture the effect of faults such as catalyst deactivation, change in porosity, and catalyst surface area.

ii. To simulate faults at various severity levels for model-based sensor placement

\subsubsection{System Level}

\section{AGR unit}

i. To identify potential faults in the AGR unit

ii. To enhance the SELEXOL process model for fault simulation 
iii. To simulate the faults and capture the dynamic data that are then used in the qualitative model-based approach for sensor network design

D. $\underline{\mathrm{CC} \text { unit }}$

i. To identify potential faults in the $\mathrm{CC}$ unit

ii. To enhance the $\mathrm{CC}$ process model for fault simulation

iii. To simulate the faults and obtain the dynamic data that are then used in the qualitative model-based approach for sensor network design

\subsubsection{Two-Tier Level}

E. Gasification Island

i. To set up an integration framework that couples and synchronizes the dynamic models of the SWGSR and the SELEXOL unit that are developed in Matlab and Aspen Plus Dynamics, respectively

ii. To simulate faults in the coupled system and obtain the dynamic data for use in the sensor placement algorithm 


\section{LITERATURE REVIEW}

\subsection{Introduction}

For sensor placement at the component level, high fidelity models of the equipment items are required. These models should be as representative of the actual equipment as possible. Therefore, they must capture the dominant mechanisms and processes taking place in the industrial-scale equipment level. A sensor network that uses a model with higher rigor is expected to require less sensors. However, since the model needs to be solved iteratively to obtain the sensor network, it should be tractable to solve them dynamically. Furthermore, the equipment models needs to be distributed so that spatial resolution of the faults can be obtained. Therefore the PDAE-based process models are appropriate at this level.

PDAE-based dynamic models of the gasifier and the SWGSR are developed. These models include sub-modes for faults. In the gasifier unit, it is important to be able to estimate the slag layer thickness and the extent of refractory degradation. In the SWGSR, it is of interest to estimate the temporal evolution of the catalyst activity so that the syngas quality can be maintained and planning for catalyst replacement can be done appropriately. 


\subsection{Gasifier}

Several gasifier models have been developed in the literature from 1-D (Kasule et al., 2012; Ubhayakar and Stickler, 1977; Wen and Chaung, 1979; Govind and Shah, 1984; Vamvuka and Woodburn, 1995; Bearth, 1996) to more complex 3-D and computational fluid dynamics (CFD) models (Chen et al., 2000; Liu et al., 2000; Wanatabe, 2006). Literature on slag layer modeling is sparse. Char particle impaction is considered to be the main mechanism for slag deposition on the wall. However, an additional mechanism for slag addition to the wall can be due to impaction of the slag droplets that get detached from the char particles. Therefore the existing literature on the process of slag formation, detachment, deposition and flow on the gasifier wall is first presented. The mechanisms for the degradation of the refractory layer are also studied in order to develop a refractory degradation model that can capture the effect of slag penetration and gasifier operating conditions.

Based on this study, a 1-D model of the slagging gasifier with refractory degradation is developed. The key processes considered in developing this model are described here.

\subsubsection{Reactions and Processes}

Several complex reactions and processes take place in the gasifier. These can be broadly outlined as devolatilization, evaporation of moisture, and homogeneous and heterogeneous reactions. In entrained-flow gasifiers, burners are designed to promote a swirling motion at the top of the gasifier that results in quick evaporation of water and subsequent devolatilization, which is then followed by combustion of the liberated volatile matter leading to a significant temperature peak. The high carbon residue formed after devolatilization is called char which undergoes combustion and gasification reactions. 


\subsubsection{Devolatilization}

Devolatilization is the release of volatile matter present in the coal matrix at high temperature. The gas released is a complex mixture containing carbon monoxide $(\mathrm{CO})$, hydrogen $\left(\mathrm{H}_{2}\right)$, methane $\left(\mathrm{CH}_{4}\right)$, carbon dioxide $\left(\mathrm{CO}_{2}\right)$, hydrogen sulfide $\left(\mathrm{H}_{2} \mathrm{~S}\right)$, nitrogen $\left(\mathrm{N}_{2}\right)$ and water vapor. Char is left behind and a carbon-rich compound called tar is also formed. The reaction is:

$$
\begin{aligned}
V M \rightarrow & \alpha_{d} \text { Tar }+\beta_{d}^{C O} \mathrm{CO}+\beta_{d}^{\mathrm{CO}_{2}} \mathrm{CO}_{2}+\beta_{d}^{\mathrm{CH}_{4}} \mathrm{CH}_{4}+\beta_{d}^{\mathrm{H}_{2}} \mathrm{H}_{2}+\beta_{d}^{\mathrm{H}_{2} \mathrm{O}} \mathrm{H}_{2} \mathrm{O}+\beta_{d}^{\mathrm{H}_{2} \mathrm{~S}_{2}} \mathrm{H}_{2} \mathrm{~S} \\
& +\beta_{d}^{\mathrm{NH}_{3}} \mathrm{NH}_{3}+\text { higher hydrocarbons }
\end{aligned}
$$

The tar further cracks due to the reaction:

$$
\begin{aligned}
\mathrm{Tar} \rightarrow & \alpha_{c} \mathrm{FC}+\beta_{c}^{\mathrm{CO}} \mathrm{CO}+\beta_{c}^{\mathrm{CO}_{2}} \mathrm{CO}_{2}+\beta_{c}^{\mathrm{CH}_{4}} \mathrm{CH}_{4}+\beta_{c}^{\mathrm{H}_{2}} \mathrm{H}_{2}+\beta_{c}^{\mathrm{H}_{2} \mathrm{O}} \mathrm{H}_{2} \mathrm{O}+\beta_{c}^{\mathrm{H}_{2} \mathrm{~S}} \mathrm{H}_{2} \mathrm{~S} \\
& +\beta_{c}^{\mathrm{NH}_{3}} \mathrm{NH}_{3}+\text { higher hydrocarbons }
\end{aligned}
$$

Devolatilization models of varying degrees of complexity exist in the literature (Anthony et al., 1976; Kobayashi et al., 1976; Niksa et al., 1991; Syamlal et al., 1992). The MGAS model of Syamlal and Bisset (1992) is used in the current work where a simple phenomenological model predicts the yields of tar and some major gas components while preserving a strict elemental balance. This model is based on data such as proximate and ultimate assays, tar composition, etc., obtained from certain lab-scale experiments that characterize the coal. A number of assumptions are made in the model so as to determine the stoichiometric coefficients of the devolatilization and cracking reactions. For example, all the sulfur in the coal is converted to $\mathrm{H}_{2} \mathrm{~S}$ while all the nitrogen is converted to $\mathrm{NH}_{3}$. Other details and kinetic parameters of the above reactions/processes are given by Syamlal and Bisset (1992).

\subsubsection{Water Vapor Evaporation}

Water is present in coal in the form of moisture associated in the carbon matrix. In the initial section of the gasifier, the moisture in the coal particle, along with the slurry water is released, and converted into water vapor (Kasule, 2012). The evaporation rate is based on the work of Rao et al. (2007). 


\subsubsection{Heterogeneous Reactions}

After the initial processes of water vapor evaporation and coal devolatilization, the char particle, consisting mostly of carbon, undergoes several heterogeneous reactions including char combustion and char-steam, char-hydrogen and char-carbon dioxide gasification. The combustion reactions rapidly take place first in the presence of high oxygen concentration. The char-steam and char-carbon dioxide gasification reactions dominate as oxygen gets depleted. Hydrogen gasification reaction rate increases as the hydrogen concentration keeps increasing along the gasifier. The main heterogeneous reactions are shown in Table 2.1 (Kasule, 2012).

Table 2.1: Solid phase reactions

\begin{tabular}{ll}
\hline Reaction & Stoichiometry \\
\hline Char combustion & $\mathrm{C}+\frac{1}{\phi} \mathrm{O}_{2} \rightarrow\left(2-\frac{2}{\phi}\right) \mathrm{CO}+\left(\frac{2}{\phi}-1\right) \mathrm{CO}_{2}$ \\
Steam gasification & $\mathrm{C}+\mathrm{H}_{2} \mathrm{O} \leftrightarrow \mathrm{CO}+\mathrm{H}_{2}$ \\
$\mathrm{CO}_{2}$ gasification & $\mathrm{C}+\mathrm{CO}_{2} \leftrightarrow 2 \mathrm{CO}$ \\
$\mathbf{H}_{2}$ gasification & $\mathrm{C}+2 \mathrm{H}_{2} \leftrightarrow \mathrm{CH}_{4}$ \\
\hline
\end{tabular}

Depending on the operating temperatures, the char-combustion reaction may be favorable towards the production of carbon monoxide or carbon dioxide. The ratio of $\mathrm{CO}_{2}$ to $\mathrm{CO}$ in the reaction products is modeled by a mechanism factor $\varphi$ which depends on the temperature at which the reaction takes place (Yagi, 1955). Higher temperature promotes the formation of carbon monoxide while lower temperature yields higher $\mathrm{CO}_{2}$ (Westbrook, 1981). The reaction kinetics for the heterogeneous reactions are discussed in Chapter 3. 


\subsubsection{Homogeneous Reactions}

The product gases from the water evaporation, devolatilization, combustion and gasification reactions participate in various homogeneous reactions. Some of the homogeneous reactions are endothermic while others are exothermic.

The following homogeneous reactions are considered in the gasifier model:

$$
\begin{array}{cl}
\mathrm{CH}_{4}+0.5 \mathrm{O}_{2} \stackrel{k_{1}}{\rightarrow} \mathrm{CO}+2 \mathrm{H}_{2} & \Delta \mathrm{H}=-35.7 \mathrm{MJ} / \mathrm{kmol} \\
\mathrm{H}_{2}+0.5 \mathrm{O}_{2} \stackrel{k_{2}}{\rightarrow} \mathrm{H}_{2} \mathrm{O} & \Delta \mathrm{H}=-242 \mathrm{MJ} / \mathrm{kmol} \\
\mathrm{CO}+0.5 \mathrm{O}_{2} \stackrel{k_{3}}{\rightarrow} \mathrm{CO}_{2} & \Delta \mathrm{H}=-283 \mathrm{MJ} / \mathrm{kmol} \\
\mathrm{CO}+\mathrm{H}_{2} \mathrm{O} \stackrel{k_{4}}{\rightarrow} \mathrm{CO}_{2} & \Delta \mathrm{H}=-41.1 \mathrm{MJ} / \mathrm{kmol} \\
\mathrm{CH}_{4}+\mathrm{H}_{2} \mathrm{O} \stackrel{k_{5}}{\rightarrow} \mathrm{CO}+3 \mathrm{H}_{2} & \Delta \mathrm{H}=+206 \mathrm{MJ} / \mathrm{kmol} \\
\mathrm{CO}+3 \mathrm{H}_{2} \stackrel{k_{6}}{\rightarrow} \mathrm{CH}_{4}+\mathrm{H}_{2} \mathrm{O} & \Delta \mathrm{H}=-206 \mathrm{MJ} / \mathrm{kmol} \\
0.5 \mathrm{~N}_{2}+1.5 \mathrm{H}_{2} \stackrel{k_{7}}{\rightarrow} \mathrm{NH}_{3} & \Delta \mathrm{H}=-46.4 \mathrm{MJ} / \mathrm{kmol} \\
\mathrm{NH}_{3} \stackrel{k_{8}}{\rightarrow} 0.5 \mathrm{~N}_{2}+1.5 \mathrm{H}_{2} & \Delta \mathrm{H}=+46.1 \mathrm{MJ} / \mathrm{kmol}
\end{array}
$$

The water-gas shift reaction (WGS) is modeled as a combination of catalytic and non-catalytic reaction rates. The rate for the catalytic reaction is obtained from Wen and Onozaki (1982) while the rate for the non-catalytic reaction is obtained from Karan et al. (1999). The kinetic parameters for other reactions and their sources are discussed further in Chapter 3. 


\subsubsection{Char Particle Characterization}

Most authors, if not all, in the literature have assumed a shrinking core model in order to characterize the kinetics on the surface of the char particle (Wen, 1968; Levenspiel, 1972; Doraiswamy and Sharma, 1984). As the reactions proceed, it is assumed that the carbon core shrinks, leaving behind a shell of solid porous ash, through which the reacting gas species diffuse (Wen and Chaung, 1979; Govind and Shah, 1984; Choi et al., 2001; Deng et al., 2008). Therefore it is assumed that the overall size of the char particle remains unchanged while the density continuously decreases as carbon is converted into gas. The overall rate is calculated by considering the combined effect of the resistances in the diffusion layer and the ash layer and the surface reaction rate. This can be seen in Eq. 2.1.

$$
\text { rate }=\frac{1}{\frac{1}{k_{\text {diff }}}+\frac{1}{k_{\text {ash }}}\left(1-\frac{1}{Y}\right)+\frac{1}{k_{s} Y^{2}}} \cdot\left(P_{i}-P_{i}^{*}\right)
$$

where $Y=\frac{r_{c}}{R_{o}} ; r_{c}$ is the radius of the unreacted core; $R_{o}$ is the original radius of the particle; and $k_{\text {diff }}, k_{\text {ash }}, k_{s}$ are the gas film diffusion coefficient, ash diffusion coefficient, and the surface reaction constants. The ash diffusion constant is obtained using the correlation given by Syamlal and Bisset (1992);

$$
k_{\text {ash }}=k_{\text {diff }}\left(\varepsilon_{\text {ash }}^{2.5}\right)
$$

where $\varepsilon_{\text {ash }}$ is the voidage of the ash layer, $P_{i}-P_{i}^{*}$ is the effective partial pressure of the $i^{\text {th }}$ component $\left(\mathrm{O}_{2}, \mathrm{H}_{2}, \mathrm{H}_{2} \mathrm{O}\right.$, or $\left.\mathrm{CO}_{2}\right)$ in the gas participating in the gasification reactions and takes into account the reverse reaction effect, $P_{i}$ is the partial pressure of component $i$, and $P_{i}{ }^{*}$ is the equilibrium pressure of reactant $i$ (Wen et al., 1979).

However, considering the high operating temperature of entrained-flow gasifiers, this characterization may not be correct. Ash from the Illinois \#6, Pittsburgh \#8, and PRB coals for most of the seams is expected to have a melting temperature lower than $1350^{\circ} \mathrm{C}$ (Vaysman, 2012). The exit temperature from the slagging, entrained flow gasifiers is typically $1350-1600^{\circ} \mathrm{C}$ 
(Kasule, 2012). The temperature immediately after the devolatilization section in which the combustion reactions take place, often exceeds the outlet temperature by a few hundred degrees. Therefore, for a major section of the gasifier, the temperature would exceed the melting point of the ash in an entrained flow gasifier.

\subsubsection{Slag Detachment and Droplet Sizes}

Formation of slag on the char particle due to ash melting has been reported in various experimental works (Quann et al., 1986; Srinivasachar et al., 1992). Since slag is highly nonwetting on the surface of carbon (Mehta, 2001; Stalder, 2010) when the ash melts, it agglomerates into one or several slag droplets rather than spreads over the surface of the char particle. Although several experimental papers (Buhre, 2006; Li, 2010; Li, 2012) have identified the formation of slag droplets on the char surface, there is hardly any work on modeling of this phenomenon.

Given the extreme operating conditions and the hydrodynamics within an entrained flow gasifier, it is likely that the droplets formed on the surface of the char particle can get separated into the bulk of the gasifier. While not a lot of research is available in the area of gasification on this topic, several papers have reported that liquid slag does exist as droplets in the bulk for combustion systems (Loehden, 1989; Yan, 2002; Seames, 2003) potentially due to "shedding" of the droplets from the char surface. Liang et al. (2012) and Wang et al. (2013) have considered deposition of slag droplets on the slag flow layer in their models; however the specific mechanism(s) by which these slag droplets are generated is(are) lacking.

Several authors have studied the slag droplets size distribution in the bulk (Loehden, 1989; Li, 2010; Buhre, 2006; Kang, 1990) in their experiments. This size distribution of the slag droplets

in the bulk of the gasifier is difficult to estimate as the mechanisms for detachment of the slag droplets are complicated and not well characterized but are believed to depend on several variables such as solids temperature, coal type, ash composition, ash quantity, coal particle size, rate of heating of char particle, feed nozzle design, profile of transport variables, and probability of attrition. The distribution can range from sizes greater than 30 micron to submicron sizes. Experimental studies using drop tube furnaces show that the size distribution of slag droplets 
also strongly depends on the mechanism by which they get detached from the char particles (Yu, 2005). Smaller, submicron slag droplets are formed predominantly due to vaporization and subsequent condensation of metal oxides (Zhang, 2009). Char particles from certain coal types can readily break up due to the rapid volumetric increase of carbon and volatile materials within the coal particle. This mechanism can also lead to the formation of slag droplets of the order of a few microns (Loehden, 1989). Larger slag droplets are formed as a result of complete coalescence where all the ash content within a coal particle coalesces to form a single slag droplet (Wang, 2013; Helble, 1990; Yu, 2005). Shedding can be a dominant mechanism when the temperature of the solids is much higher than the melting point of the ash. The size of the liberated slag droplets also depends on the conversion rate of the char particles. During rapid reaction, the char surface recedes rapidly and the molten ash minerals do not have sufficient residence time on the surface to coalesce. It is found that the separation of individual included mineral matter or partially coalesced mineral inclusion results in droplet sizes between 1-20 microns due to shedding or char fragmentation (Buhre, 2006). Therefore, a particle size distribution (PSD) of detached slag droplets would be expected in the bulk.

\subsubsection{Deposition onto Slag Layer}

A common assumption made about the mechanism of addition of slag to the slag flow layer is that it only takes place due to char particle impaction (Seggiani, 1998; Lee, 2014; Bockelie, 2002; Benyon; 2002). All these papers have considered that a fraction of the char particles hits the flowing slag layer on the wall of the gasifier. A fraction of these char particles stick to the wall and continue to react. As a result, the ash contained in these char particles melts contributing to the slag layer.

Some authors have studied and incorporated capture efficiencies of the char particles (Yong, 2012), which is the ratio of the char particles that are captured in the slag flow layer to the total particles impacting the layer. The burnout process of the captured char particle has also been studied by developing models for calculating the rate of reaction of these particles (Wang, 2007; Wang, 2009). 
However, deposition of slag due to char impaction may not be the only mechanism by which slag gets added to the flowing slag layer on the wall. As the operating temperature of a slagging gasifier is generally much higher than the ash melting temperature, it is likely that molten ash may be liberated into the gasifier bulk ( $\mathrm{Li}, 2010$; Li, 2012; Buhre, 2006; Kang, 1990) as mentioned in the earlier section. In this work, a shrinking particle model (SPM) is proposed that captures the formation of slag droplets due to reaction of char particles. A fraction of these slag droplets can also get deposited on the wall in addition to the char particles.

Slag deposition flux to the wall is difficult to model and a number of assumptions are often made in the literature. Deposition flux is typically assumed to be constant (Seggiani, 1998; Ye, 2015) or set to a fraction of the total solid flow rate entering the gasifier (Monaghan, 2012). Another common assumption is to consider a fixed profile for slag deposition along the wall (Yang, 2011) during steady state and dynamic simulations. Obviously, these assumptions are somewhat arbitrary and difficult to justify especially during transient operation of the gasifier. A number of authors have developed CFD models (Yong, 2012; Lei, 2013; Bockelie, 2002) that track particle trajectories to calculate the net amount of slag deposition on the wall. However, it is computationally intractable to extend these rigorous models to perform dynamic simulations on a commercial-scale gasifier.

Given that the objective is to develop a tractable model that can be solved dynamically and used for fault simulation, a 1-D model for the gasifier will be developed. An analytical expression is required to be included in this model for calculating the velocity of the char particles and slag droplets. Experimental and computational works are available in the open literature where the transport and deposition of solid particles or liquid droplets suspended in a flowing fluid are studied. The results are usually presented in the graphical form of a dimensionless velocity, $V_{d e p+}$, as a function of dimensionless relaxation time, $\tau_{+}$. (Wood, 1981; Ahmadi, 1996; Wang, 2007; Guha, 2008; Wang, 2009) as seen in Figure 2.1. 


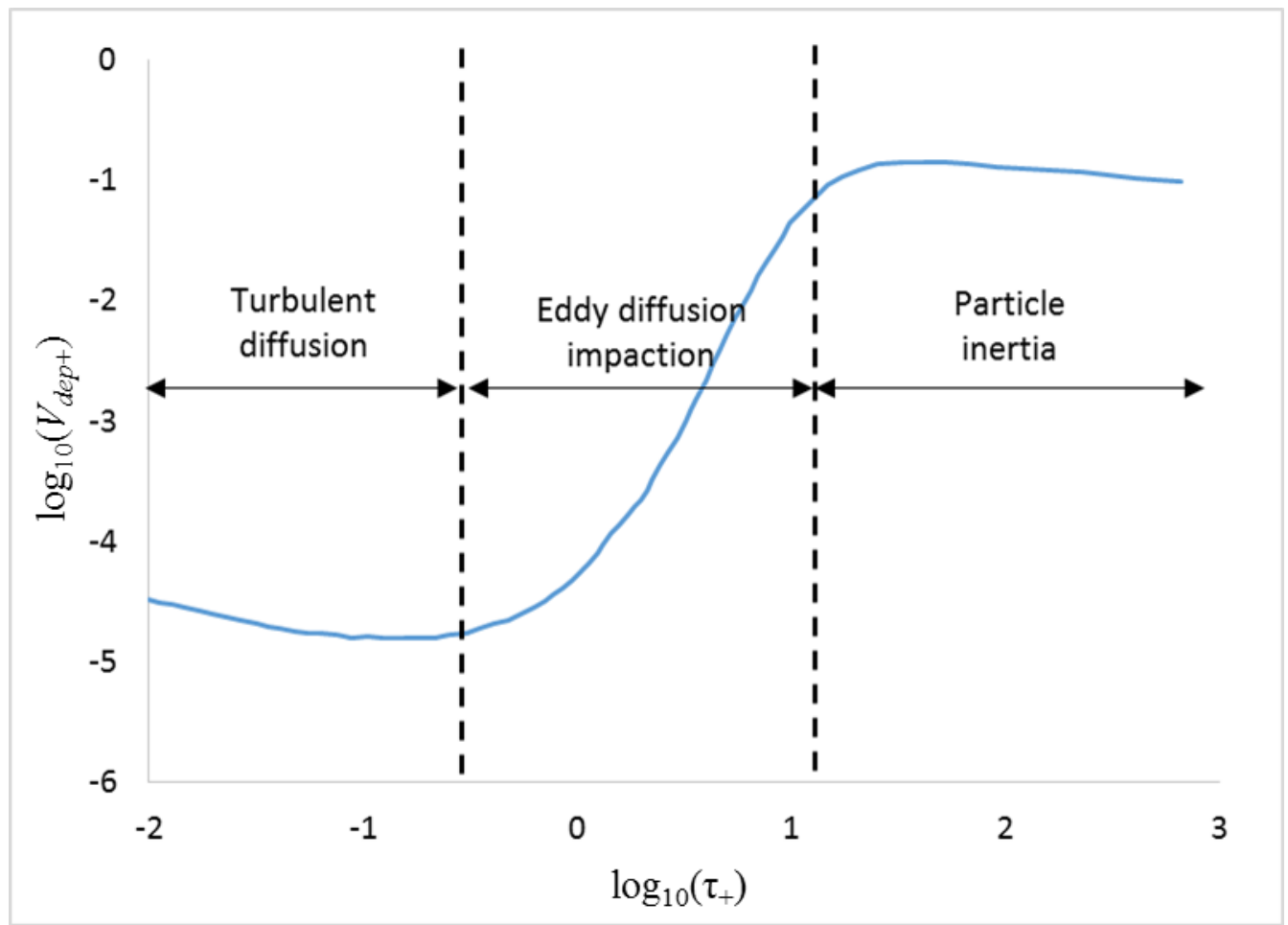

Figure 2.1: General plot showing the relation between the $V_{d e p+}$ and $\tau_{+}$redrawn

The dominant deposition mechanism(s) and deposition velocity can vary greatly depending on the value of $\tau_{+}$. Particles that are a few microns in size fall in the regime where the deposition is mainly due to turbulent diffusion. In this case, the deposition velocity is small. Larger particles with higher relaxation time deposit due to eddy diffusion impaction. In this regime, the deposition velocity increases rapidly with increase in particle size. Deposition velocity levels out as the relaxation time increases further. In this third regime, particle inertia is the dominant deposition mechanism.

A few authors (Ahmadi, 1996; Wang, 2007; Wang, 2009) have used correlations between the dimensionless relaxation time and dimensionless velocity to calculate the deposition rate in combustors and gasifiers. In the present study, the correlations proposed by Wood (1981) are used to calculate the deposition velocity of the char particles and the slag droplets of different sizes. 


\subsubsection{Slag Flow Layer}

Perhaps one of the first attempts to model the slag flow layer in the gasifier was made by Seggiani et al (1998). This 1-D model of the slag has been used by several authors for modeling the slag flow layer in gasifiers and combustors. A few, more complex slag flow models have been proposed in the literature which are 2-D models (Lie, 2013; Bockelie, 2002) that have been applied to 3-D combustor or gasifier models. However, for the purpose of the present work, the model developed by Seggiani et al (1998) is used for modeling the slag flow layer.

\subsubsection{Refractory Degradation}

Refractory degradation is one of the leading issues that impact economic viability of the entrained-flow gasifiers (Clayton, 2002). Replacement of refractory bricks is done from every 3 months to 2 years. Refractory replacement is expensive and also results in the downtime of the entire power plant (Sundaram, 2009). Non-destructive tests to assess the life of the refractory layer online are not available and temperature sensors embedded in the refractory layer do not survive in the harsh environment of the gasifier. Therefore, refractory bricks to be used in the gasifier are often experimentally tested or monitored after being used in the field to identify their short comings and mechanism of failure (Bennett and Kwong, 2011).

Several mechanisms have been identified that contribute to the degradation of the refractory layer (Bennett and Kwong, 2011). The slag, deposited on the refractory wall of high temperature gasifiers can directly interact with the refractory layer through corrosion and erosion mechanisms. The slag can also penetrate into the refractory and change the material properties of the brick resulting in spalling of the refractory due to compressive and tensile mechanisms (Williford, 2008).

Refractory degradation can also take place in the absence of slag through mechanisms such as creep and thermal fatigue (Bennett and Kwong, 2011). These are generally slow processes, taking place over periods of months; however they could become dominant in high temperature operation. They lead to formation of micro-cracks that can change material properties such as Young's modulus, and maximum tensile stress resulting in the weakening of the material. 
Thermal shock is an example of a fast mechanism where sudden change in temperature could result in build-up of stresses near the hot face surface.

Some of these processes are difficult to model due to lack of experimental data available in the literature. Corrosion is one of the main mechanisms by which refractory degradation takes place due to slag interaction, but modeling this requires the chemical kinetics to be available. Since the process is slow and may take several months, experimental work often simulates this at more extreme conditions in the lab and rates developed from such experiments may not be accurate. A few authors have used formulations in terms of a general reaction rate using a concentration gradient (Lui et al., 2008) or rate of loss of materials (Chen, 1984), but these models do not capture the true dynamics of the system or the effect of change in composition of both the slag and the refractory. Furthermore, these reactions are solid state reactions and therefore are generally difficult to characterize. For example, the refractory is a combination of several mineral phases that may change under different operating conditions as can be seen from the equilibrium phase diagrams in the literature. Creep and thermal fatigue may be dominant mechanisms for refractory degradation at high temperatures; however experimental data to help formulate these models accurately are scarce. Creep can be helpful to some extent, as it results in stress relaxation in the refractory which can be modeled using empirical equations for creep rate available in the literature. However, the process by which creep and thermal fatigue affect the material constants and cause degradation is unclear in the literature. 


\subsubsection{Refractory degradation models}

Although refractory degradation is identified as an important concern in high temperature gasifiers, the literature on modeling in this topic is scarce. Earlier work include that of Chen (1984) who developed a thermomechanical model of the refractory of a slagging gasifier and considered the effects of temperature change, on the stress buildup in the refractory due to the thermal expansion. Creep was also considered in the model which results in stress relaxation. The effect of slag was considered using a corrosion model. A finite element analysis considering a displacement method was used to calculate the stress in their work.

Work in the area of modeling refractory degradation due to slag effects has been done by Williford et al. (2008). They identified two mechanisms by which slag penetration could result in refractory degradation and eventually spalling viz. compressive and tensile spalling (Williford, 2008). These authors also state that, compressive spalling takes place due to the exchange of ions between the slag penetrated region and the slag free region in the chrome refractory. $\mathrm{Fe}^{3+}$ ions from the slag replace the $\mathrm{Cr}^{3+}$ ions in the refractory matrix (Williford, 2008). Due to the bigger size of the former ions, the refractory penetrated region begins to swell leading to the build-up of stress. As the slag continues to penetrate into the refractory, the thickness of the slag penetrated region increases to buckle until which it spalls. Tensile spalling on the other hand is the result of the migration of the $\mathrm{Cr}^{3+}$ ions out of the refractory brick (Williford, 2008). The loss of the $\mathrm{Cr}^{3+}$ ions results in the refractory material results in volume shrinkage of the high chrome refractory and the formation of cracks. The cracked segments are removed by the flowing slag layer resulting in a spall.

Modeling of materials is often done using finite element analysis that calculates the stress and displacement in the system. Due to the requirement of a dynamic process model that can be solved within reasonable amount of time, a model using analytical equations for the stress may suffice. To capture the thermal and mechanical effects on the stress, a thermo-elastic model for the refractory material can be developed (Hetnarski and Eslami, 2010) for a cylindrical system. Multi-layered systems are studied to obtain correct interface conditions to model the stresses in the refractory layers. 


\subsection{Sour Water Gas Shift Reactor}

The water gas shift reaction is a well-studied equilibrium reaction where several models of the sweet shift reactor have been developed (Bell and Edgar, 1991; Ding and Chan, 2008; Adams and Barton, 2009; Francesconi et al., 2007; Guinta et al., 2006). Giunta et al. have performed an extensive study on a 2-D heterogeneous dynamic model and validated with experimental data. In their work, consideration for the intra-particle mass transfer limitations by the definition of effectiveness factor is included. Using small catalyst diameters (below $0.8 \mathrm{~mm}$ ), they are able to obtain results when compared to the commercial-scale reactors. Adams and Barton (2009) have developed a 1-D heterogeneous dynamic model and validated with the work of Choi and Stenger (2003). Steady-state models of WGS reactor and their validation with the experimental data have been reported in the work of Ding and Chan (2008) and Chen et al. (2008). Francesconi et al.(2007) have discussed optimization of the reactor at steady-state condition.

Most of the efforts in modeling the WGS reactors have focused on the sweet shift process where several catalysts at different conditions have been studied (Gunawardana et al., 2009; Levent, 2001; Lim et al., 2009; Sun et al., 2005; Amadeo and Laborde, 1995). In comparison to the vast amount of work on the sweet shift catalysts, the amount of work on the sour shift catalysts is very little. A few experimental works can be found that have investigated the performance of the sour shift catalysts and have performed kinetic studies in the presence of a sulfiding agent such as $\mathrm{COS}$ or $\mathrm{H}_{2} \mathrm{~S}$, which are typically present at reasonable concentrations in the syngas obtained from a coal-fired gasifier (Hou et al., 1983; Li et al., 1999; Hla et al., 2011; De la Osa et al., 2011; Andreev et al., 1999; Hakkarainen et al., 1993). Additionally, computational models developed for sour shift reactors are rare. Bell and Edgar (1991) have developed 1-D pseudohomogeneous model of a reactor that is filled with the $\mathrm{Co} / \mathrm{Mo}$ based catalyst, which is similar to the catalyst used in this work with the exception that the catalyst used in this work is promoted with cesium (Berispek, 1975). Although they verified their steady-state and dynamic model with experimental results, their lab-scale reactor model cannot be scaled up to an industrial reactor due to their assumptions that are exclusive to lab-scale models and under-predict the results for industrial-scale reactors. In their work, they have ignored the momentum balance while modeling their reactor; therefore, information on the pressure drop across the reactor is not included. Pseudo-homogeneous models are sufficient only when intra-particle heat and mass transfer 
limitations are negligible, which is not the case for an industrial-scale reactor (Jakobsen, 2008). Since a typical industrial-scale sour shift reactor is filled with larger catalyst particles and operates at higher pressures compared to catalyst particle size and operating pressure in experimental studies, their model cannot be used for studying the performance of the reactor under industrial conditions.

In almost all experimental and computational papers, COS hydrolysis reaction have not been studied together with the WGS reactions. It must be noted that a significant conversion of COS in the shift reactor(s) is desired so that the resulting $\mathrm{H}_{2} \mathrm{~S}$ can be captured in the AGR unit to satisfy the overall specifications on sulfur emission (Bhattacharyya et al., 2010). The COS hydrolysis reaction would be expected to occur on the sour-shift catalysts since the typical sourshift catalysts use Co/Mo supported on alumina, and alumina has been reported to catalyze the COS hydrolysis reaction (Fiedorow et al., 1984; George, 1974; Hoggan, 1994).

\subsection{Conclusion}

The literature review presented in this section shows various works that have looked at different aspects of the gasifier and SWGSR models. In the gasifier modeling work, a shrinking particle model is used to characterize the char particle since most of these models do not consider the slagging phenomenon. The authors who have developed models of the slag layer assume that only char particles impact the slag layer and therefore no changes are made to the characterization. However, since the bulk temperatures in entrained flow gasifiers can easily exceed the melting point of ash for a large section in the gasifier, it is more likely that the ash would melt and remain a solid shell around the char particle. Mathematical models of slag droplet formation and detachment are largely missing in the open literature. In the present work, a novel hybrid shrinking core-shrinking particle model is developed to address the issue of slag formation in the bulk of the gasifier and more accurately characterize the heterogeneous reactions occurring on the char particle surface. The slag droplet formation and detachment processes are also considered in this work. In this work, in addition to impaction of char particles, the impaction of slag droplets is also considered to contribute to the slag deposition process. This additional mechanism has not been considered in the literature. Very few papers 
have developed a dynamic model of a slagging gasifier in the literature. In the current work a dynamic 1-D model of a slagging gasifier is developed that includes sub-models for the formation and detachment of slag droplets and the transport and deposition of char particles and slag droplets onto the wall to form a slag layer. In addition, compressive spalling and thermal and mechanical stress along with stress build-up due to slag penetration are studied and modeled. The model also provides a framework so as to add additional mechanisms of refractory degradation in the future.

A 1-D dynamic model of the SWGSR is developed with sulfur tolerant catalyst. The SWGS reaction is considered together with the COS hydrolysis reaction, which is not done in the literature. The reaction kinetics for the COS hydrolysis reaction are obtained by performing data reconciliation from experimental work. The developed model is modified to simulate an industrial scale SWGSR system in the IGCC and simulate catalyst based faults.

The modeling of the gasifier using the hybrid shrinking core shrinking particle model and considering the process of slag droplet formation and detachment is described in Chapter 3. Chapter 4 describes the slag droplets in greater details and discusses modeling the slagging gasifier model with the slag layer. The refractory degradation model is covered in Chapter 5. Chapter 6 describes the modeling of the SWGSR system. 


\section{GASIFIER WITH SLAG MODELING}

\subsection{Introduction}

The gasifier is considered as the heart of the IGCC plant. Coal is converted to syngas, mainly CO and hydrogen, in the gasifier. The entrained-flow gasifiers achieve high conversion of carbon, but operate at very high temperatures. Therefore, the gasifier is selected for fault simulation and sensor placement as part of this project.

Two main faults that could occur in an entrained flow gasifier during operation are build-up of slag layer on the hot face of the gasifier and refractory degradation. An increase in the thickness of the slag layer can eventually shutdown of the gasifier. Refractory degradation occurs at a much slower time scale than the slag layer but can also lead to shutdown. For improving the availability of the gasifiers and for maintenance planning, it would be very beneficial to estimate the extent of these faults. This information can eventually be used to develop operational strategies.

In order to simulate the faults in the gasifier, a distributed model of a gasifier is required. In this chapter, a model for the gasifier unit is developed that captures the process of slag formation and detachment into the bulk. From the discussion in Section 2.3, a shrinking-particle model seems more physically correct for the region where the gasifier bulk temperature well exceeds the ash melting temperature. However, in the early region of the gasifier, where the bulk temperature remains lower than the ash melting temperature, a shrinking-core model seems more appropriate. In this chapter, a novel first principles, one-dimensional, non-isothermal, pressure-driven dynamic model for a downward-firing, entrained-flow, slurry-fed, oxygen-blown (GEE-Texaco type) gasifier using a hybrid shrinking-core-shrinking-particle reaction model is first discussed. A novel sub-model for slag formation on the char surface and detachment into the bulk is formulated and is also covered. The developed model is then used to study the effects of various key variables on the slagging process and compare them with the results for the traditional shrinking-core models. Complete coalescence and slag detachment scenarios are simulated and 
the impact on slag build-up on the char surface and slag droplet number density in the gasifier bulk is studied.

\subsection{Model Description}

The shrinking-core model used in this work has been previously presented by Kasule et al. (2012, 2014) and is used for the early region of the gasifier where the bulk temperature is below the ash melting temperature. Details of that model can be found in the work of Kasule et al. (2012). It should be noted that in entrained-flow gasifiers, burners are designed to promote a swirling motion at the top of the gasifier that results in quick evaporation of water and the subsequent devolatilization step followed by combustion of the liberated volatile matter leading to a significant temperature peak. From that region to the exit of the gasifier, the solids temperature remains well above the melting point of ash. Therefore the shrinking particle model is applied to that region. Figure 3.1 shows the regions where shrinking core and shrinking particle models are applied.

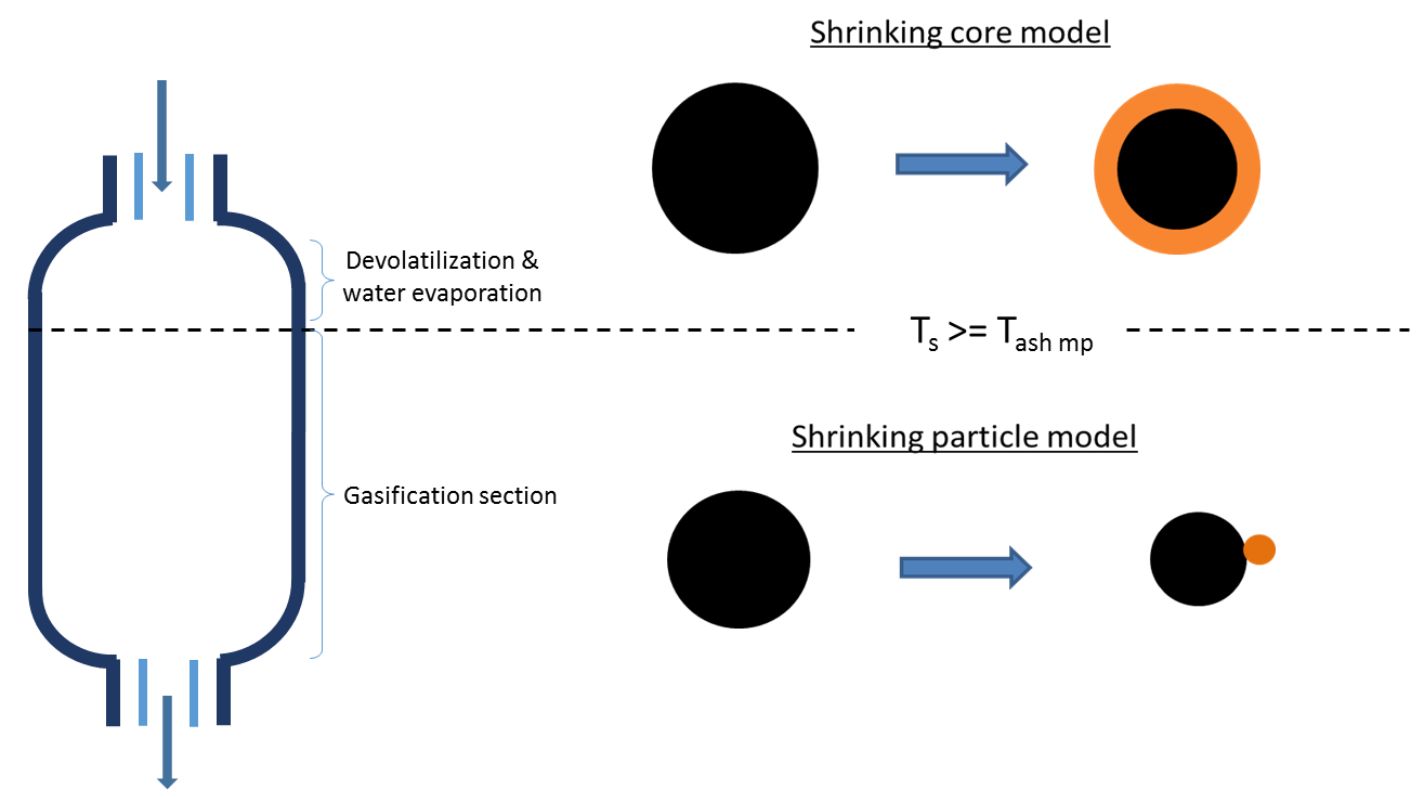

Figure 3.1: Hybrid shrinking core shrinking particle (HSCSP) model

The shrinking particle model presented in this chapter is novel and to the best of the knowledge of the authors, has never been proposed for the gasifier. The shrinking particle model is onedimensional and considers both the solid and gas phases. Mass, momentum and energy equations 
are written for both phases. The molten slag in the bulk, as well as that attached to the char particle, is considered to be part of the solid phase. The dimensions of the gasifier are based on the GEE- Texaco gasifier (TECO final technical report, 2002). A rigorous model for the heat balance on the gasifier wall is also considered. Gas recirculation is also modeled, similar to the shrinking core model (Kasule, 2012), to mimic the swirling effect produced by the burners.

\subsubsection{Assumptions}

The entrained flow gasifier is intended to be used as a process models in order to perform dynamic simulations and fault simulations. Therefore, it is required to be rigorous at the same time computationally tractable. In order to do so, the following assumptions have been made in developing the shrinking-particle model:

1. Char particles and slag droplets are spherical.

2. Radial distribution of char particles is uniform.

3. Slag separation occurs uniformly for all char particles.

4. No particle-particle interaction; system is assumed to be dilute in solids.

5. No slag deposition is considered in the present work.

6. Three discrete detachment diameters are used in this work. These are 5, 10 and $15 \mu \mathrm{m}$. A complete coalescence case is also considered.

7. The char particle and detached slag droplet velocity are assumed to be equal and solved for using a single momentum balance equation. For entrained flow gasifiers, it is found that the volume fraction of solids is very small, less than one percent (Shah et al., 1984). Furthermore, the solid particle sizes considered are $<100$ microns in diameter and for such systems, the differences in gas and solid velocities are found to be very small (Kumar et al., 2012). The detached slag droplets are smaller than the char particles and therefore would tend to flow at the gas velocity. The solid phase velocity calculated on the basis of the char particle and slag droplets can be expected to be even closer to the gas velocity and therefore the error in calculating the solid velocity using a single momentum balance equation is assumed to be small. 


\subsubsection{Hybrid Shrinking Core Shrinking Particle Model}

Due to the very high operating temperature of the entrained-flow gasifiers, it is expected that the ash on the char particle gets molten. Since slag is highly non-wetting on the surface of carbon, the molten ash, or slag, is likely to agglomerate into one or several droplets over the surface of the char particle. If the slag exists in the form of droplets on the char surface rather than as a solid shell around the unreacted char particle, then the widely-used shrinking core model (SCM) does not seem physically correct. Rather, a shrinking particle model (SPM) would be a more physically realistic representation. Unlike the shrinking-core model that assumes the diameter of the char particle to be constant, the shrinking-particle model considers the char particle to shrink while the slag droplet(s) would build up on the particle's surface. Eventually the slag droplets may detach from the char surface moving into the gasifier bulk. More included mineral matter gets exposed on the surface leading to the formation of new droplets. This suggested mechanism is shown in Figure 3.2.

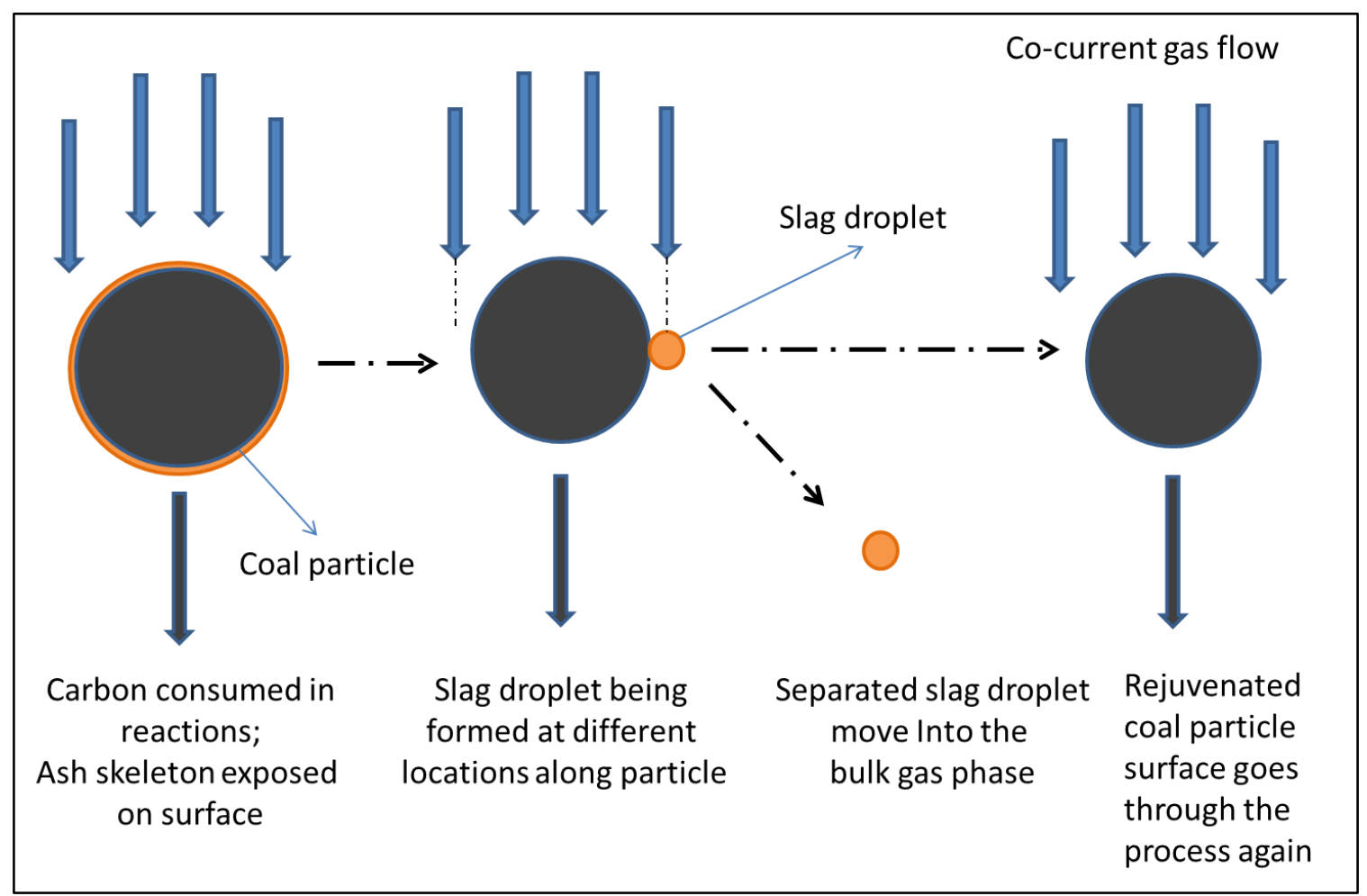

Figure 3.2: Slag formation and detachment 


\subsubsection{Mass Balance Equations}

Both the solid and gas phases are modeled as continuous phases. A particle model is developed to account for the slag droplets that are attached to the char particles and for the detached slag droplets that exist in the bulk and is integrated with the continuous phase model. The gas phase

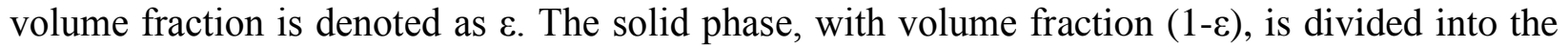
volume fraction of the slag droplets in the bulk, given by $\varepsilon_{\mathrm{sd}}$ and the volume fraction of the charslag system, i.e., char particles with the slag droplets attached to them and is denoted as (1- $\left.\varepsilon_{\text {sd }}\right)$. The attached slag droplet to the char particles is accounted for by the volume fraction $\varepsilon_{\text {sa. }}$. The notations are shown in Figure 3.3.

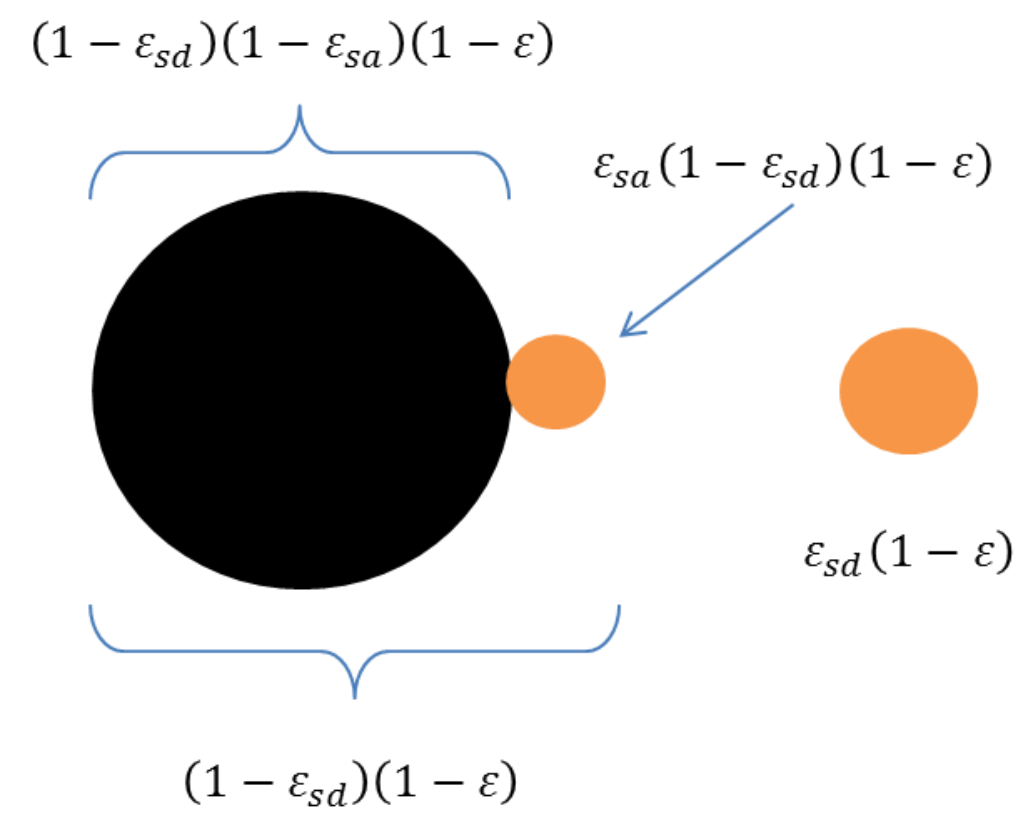

Figure 3.3: Schematic of solid phase consisting separated slag, attached slag, and char particle 


\subsubsection{Continuous Phase Model}

The continuous phase model is developed for the gas phase and overall solid phase. Additional conservation equations are written for slag droplets that are attached to the char particles as well as for the slag droplets that are detached.

\section{Mass conservation equations}

Eq. 3.1 shows the overall solid phase mass conservation. In this equation, the second term on the right hand side represents the solids loss due to reaction where the reaction rate has been defined with respect to the char particle. As seen in Figure 3.3, the volume fraction corresponding to the char particle is $(1-\varepsilon)\left(1-\varepsilon_{s a}\right)\left(1-\varepsilon_{s d}\right)$. The solids can also be lost from the bulk due to deposition on the gasifier wall as given by the third term in Eq. 3.1. Eq. 3.2 shows the gas phase mass conservation equations.

$$
\begin{gathered}
\frac{\partial\left(\rho_{s, a v g}(1-\varepsilon)\right)}{\partial t}=-\frac{\partial\left(\rho_{s, a v g}(1-\varepsilon) U_{s}\right)}{\partial x}-(1-\varepsilon)\left(1-\varepsilon_{s a}\right)\left(1-\varepsilon_{s d}\right) \Gamma_{s-g}-\frac{4 M_{\text {slag dep. }}}{D_{i}} \\
\frac{\partial\left(\rho_{g} \varepsilon\right)}{\partial t}=-\frac{\partial\left(\rho_{g} \varepsilon U_{g}\right)}{\partial x}+(1-\varepsilon)\left(1-\varepsilon_{s a}\right)\left(1-\varepsilon_{s d}\right) \Gamma_{s-g}-m_{r g}+m_{m g}
\end{gathered}
$$

In Eqs. 3.1 and 3.2, $\rho_{s, a v g}$ and $\rho_{g}$ are the average solid and gas densities, $U_{s}$ and $U_{g}$ are the solid and gas velocities, $\Gamma_{s-g}$ is the sum of all heterogeneous reactions, $M_{\text {slag dep. }}$ is the mass deposition rate of slag onto the gasifier wall in the control volume and $D_{i}$ is the internal wall diameter. To model and understand the process of slag formation and detachment clearly, the deposition of char onto the gasifier wall is not considered in the present chapter.

The recirculation effect in the gas phase is captured by the terms $m_{r g}$, which is the mass of gas that leaves the control volume (CV) because of recirculation, and $m_{m g}$, which is the mass of gas that gets added to a $\mathrm{CV}$ due to recirculation. These terms are calculated by the following equations:

$$
\begin{aligned}
& m_{r g}=\dot{m}_{\text {recir }} / A_{R} L_{2} \\
& m_{m g}=\dot{m}_{\text {recir }} / A_{R} L_{1}
\end{aligned}
$$




$$
\dot{m}_{\text {recir }}=\alpha \dot{m}_{\text {in }}
$$

where $A$ is the cross section area, $L_{2}$ is the length of the zone from where the recirculating gas is removed and $L_{1}$ is the length of the zone where the gas is added into the bulk gas stream, $\alpha$ is the recirculation ratio and $\dot{m}_{i n}$ is the inlet gas stream. A schematic of the recirculation model is shown in Figure 3.4 .

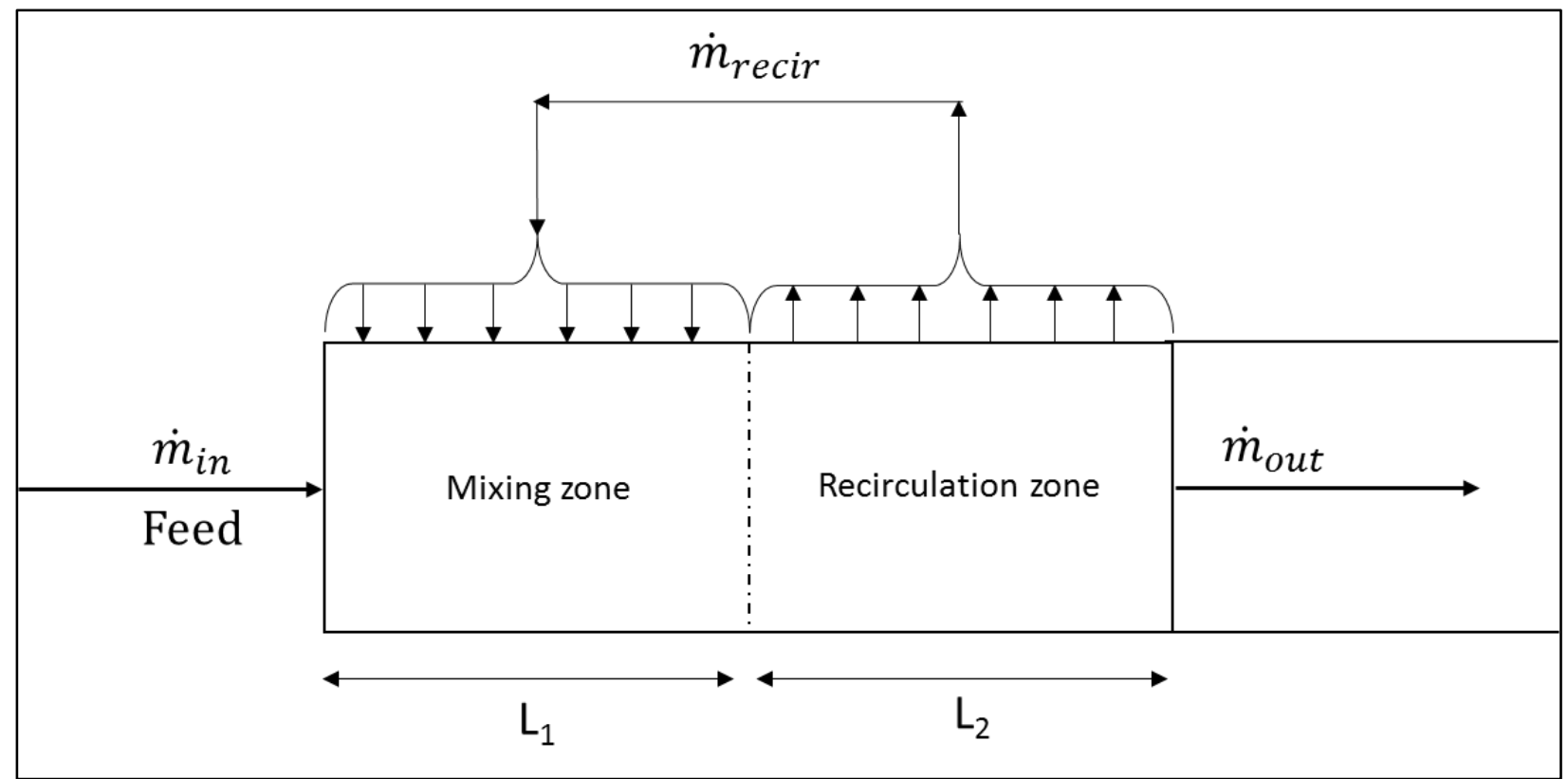

Figure 3.4: Schematic of the recirculation model

Eqs. 3.6 and 3.7 show the species conservation equations for the solid and gas phases, respectively.

$\frac{\partial\left((1-\varepsilon)\left(1-\varepsilon_{s a}\right)\left(1-\varepsilon_{s d}\right) \rho_{c h} X_{s, j}\right)}{\partial t}=-\frac{\partial\left((1-\varepsilon)\left(1-\varepsilon_{s a}\right)\left(1-\varepsilon_{s d}\right) \rho_{c h} U_{s} X_{s, j}\right)}{\partial x}+(1-\varepsilon)\left(1-\varepsilon_{s a}\right)\left(1-\varepsilon_{s d}\right) r_{s, j}$

$$
\frac{\partial\left(\varepsilon \rho_{g} y_{g, i}\right)}{\partial t}=-\frac{\partial\left(\varepsilon \rho_{g} U_{g} y_{g, i}\right)}{\partial x}+\varepsilon r_{g, i}-m_{r g} y_{g, i}+m_{m g} y_{g, i, a v g}
$$

The volume fraction corresponding to the char particle volume, shown in Figure 3.3 is used in Eq. 3.6 for each of the terms. The last two terms in Eq. 3.7 correspond to the recirculation of gas species out of and into the control volume similar to the overall gas balance equation. $y_{g, i}$ is the

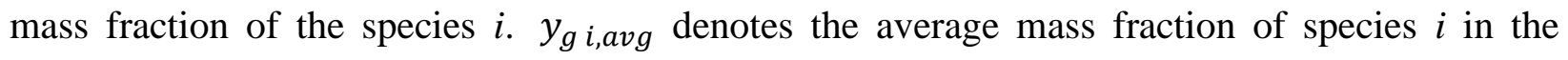


circulating flow. Details of the recirculation model can be found in the work of Kasule et al (2012).

The gas phase density is calculated by assuming ideal gas law in the form given by Eq. 3.8.

$$
\rho_{g}=\frac{P}{R T_{g}} \cdot \frac{1}{\sum_{i=1}^{N}\left(y_{i} / M W_{i}\right)}
$$

In Eq. 3.8, $N$ is the total number of gaseous species and $y_{i}$ and $M W_{i}$ are the mass fraction and molar weight of the $i^{\text {th }}$ gaseous species.

\section{Mass conservation of attached slag}

Eq. 3.9 represents the mass conservation of attached slag.

$\rho_{s l} \frac{\partial\left((1-\varepsilon)\left(1-\varepsilon_{s d}\right) \varepsilon_{s a}\right)}{\partial t}=-\rho_{s l} \frac{\partial\left((1-\varepsilon)\left(1-\varepsilon_{s d}\right) \varepsilon_{s a} U_{s}\right)}{\partial x}+(1-\varepsilon)\left(1-\varepsilon_{s a}\right)\left(1-\varepsilon_{s d}\right) \Gamma_{s-g} \omega_{a s h}-$

$p_{s l} M_{c d}$

Mass is added to the attached slag droplet as ash melts during heterogeneous reactions as represented by the second term on the right hand side. The third term accounts for separation of the slag droplet into the bulk, where, $p_{s l}$ denotes number of slag droplets separated per unit volume per unit time and $M_{c d}$ is the mass of the slag droplet of the critical diameter. It is noted that, $p_{s l}$ will be zero for a $\mathrm{CV}$ if no slag detachment has taken place in that $\mathrm{CV}$.

\section{Mass conservation of detached slag}

As mentioned before, it has been assumed that as the slag droplet size exceeds some critical diameter, it gets detached from the char particle. Therefore, the slag separation is not a continuous process. With this assumption, the mass conservation equation for detached slag droplets is given as Eq. 3.10.

$$
\rho_{s l} \frac{\partial\left((1-\varepsilon) \varepsilon_{s d}\right)}{\partial t}=-\rho_{s l} \frac{\partial\left((1-\varepsilon) \varepsilon_{s d} U_{s}\right)}{\partial x}+p_{s l} M_{c d}-\frac{4 M_{\text {slag dep. }}}{D_{i}}
$$




\subsubsection{Particle Model, Slag Formation and Detachment}

For the continuous model, it is important to know the magnitude of the terms $p_{s l}$ as well as the amount of slag that gets deposited on the wall. In this chapter, it has been assumed that the amount of slag deposited on the wall is zero, i.e. all slag exits through the bulk of the gasifier in order to make an unbiased comparison of results from the traditional shrinking-core model. However, for calculating the term $p_{s l}$, a particle model is required. This model tracks the growth of the slag droplets on the char particle and helps to identify the locations of detachment and the detachment rate. Then this model is used to track the number density of slag droplets and char particles in the gasifier bulk.

Continuum phase domain

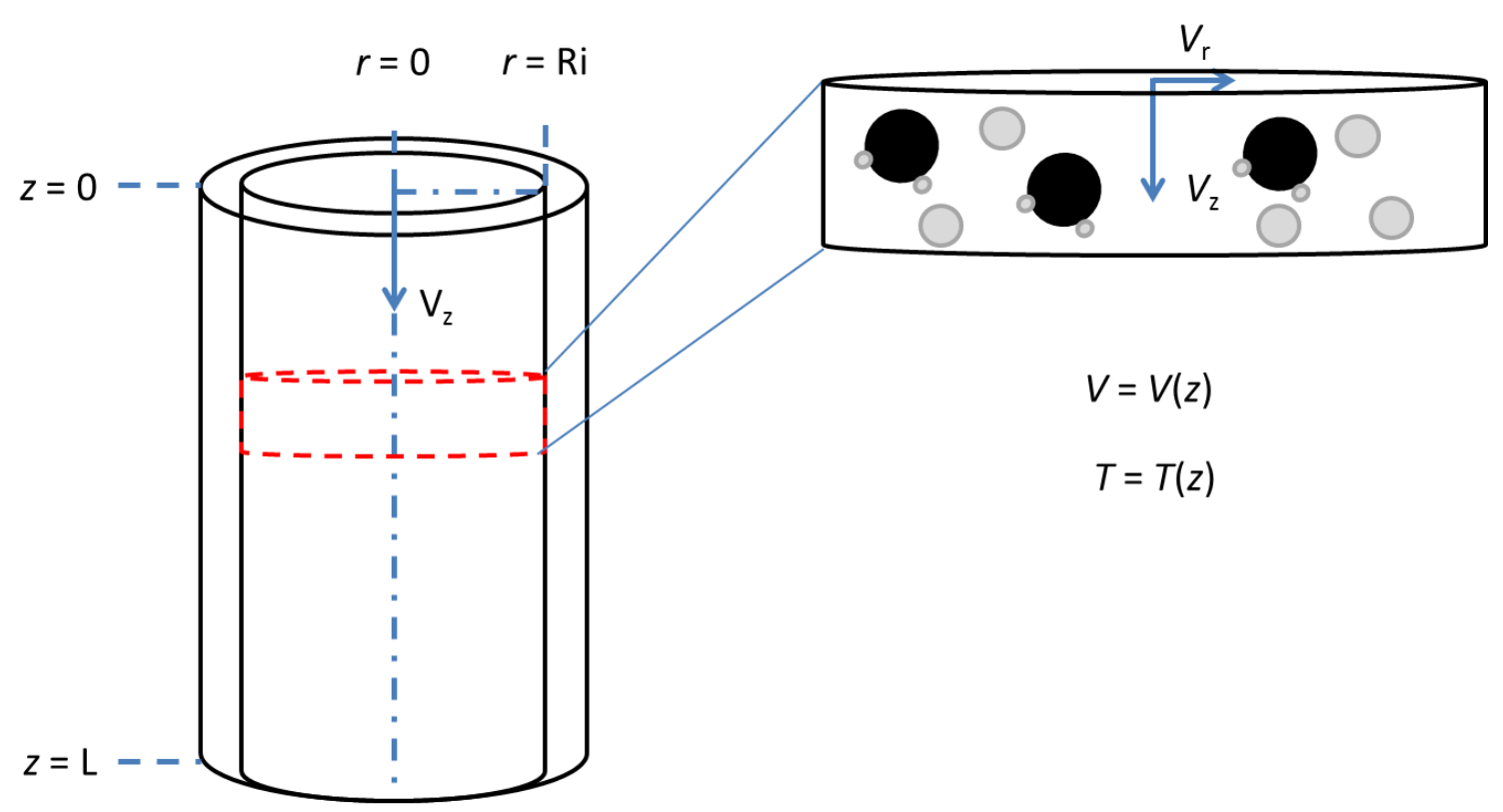

Figure 3.5: Continuum phase domain for solid and gas integrated with the particle phase domain

Figure 3.5 shows that on an overall scale, a continuum description is used for the gas and solid mass balance. In order to account for the number of slag droplets, a particle phase model is used under the continuum description such that the overall mass balance of the continuum is still satisfied. 


\section{Slag detachment model}

The slag detachment is not a continuous process as pointed out. Therefore, algebraic equations are developed to model this process. Figure 3.6 shows the schematic of the slag formation and detachment model. The term $p_{s l}$ is calculated from Eq. 3.11:

$$
\begin{gathered}
p_{s l, i} \tau_{i}=w_{s l, i} N_{c h, i} \\
\tau_{i}=\frac{\Delta x}{U_{s, i}}
\end{gathered}
$$

In Eq. 3.12, $\tau_{i}$ is the residence time of the solids in the $i^{\text {th }}$ control volume $(\mathrm{CV}) ; w_{s l, i}$ is the number of slag droplets generated per char particle in the $\mathrm{CV}$; and $N_{c h, i}$ is the number of char particles per unit volume in the CV. The term $w_{s l, i}$ is calculated from Eq. 3.11.

$$
w_{s l, i}=\text { floor }\left(\frac{\omega_{a s h} \Gamma_{s-g, i} V_{c h, i} \tau_{i}+M_{s r, i-1}}{M_{c d}}\right)
$$

where, $\omega_{a s h}$ is the ratio of ash to carbon mass fraction. As can be seen, until the attached slag mass exceeds the critical slag mass, the value of $w_{s l, i}$ is zero. It is possible that if the critical mass is small or the reaction rate is very high, multiple slag droplets can form in a single control volume.

It should also be noted that in the shrinking-particle model, the mass fraction of the ash and carbon in the char remain unchanged due to assumption of homogeneous composition. Therefore, the $\omega_{a s h}$ term is constant. $V_{c h, i}$ is the volume of the char particle in the CV. $M_{s r, i-1}$ denotes the residual mass of the slag droplet from the previous control volume. The residual mass of the slag droplets accounts for the mass of the slag droplets attached to the char particles from previous CVs. It should be noted that this term captures the mass of the slag droplets that did not get separated. Finally $M_{c d}$ is the critical mass of the slag droplet. 
The slag droplet formation is described by Eq. 3.14, where $M_{s r, i}$, is the amount of slag left behind after separation of the slag droplet.

$$
w_{s l, i} V_{c d} \rho_{s l, i}+M_{s r, i}=\omega_{a s h} \Gamma_{s-g, i} V_{c h, i} \tau_{i}+M_{s r, i-1}
$$

The first term on the left hand side of Eq. 3.14 is the amount of slag that became separated in the $i^{\text {th }} \mathrm{CV}$. The first term on the right hand side represents the amount of slag generated in the $i^{\text {th }} \mathrm{CV}$.
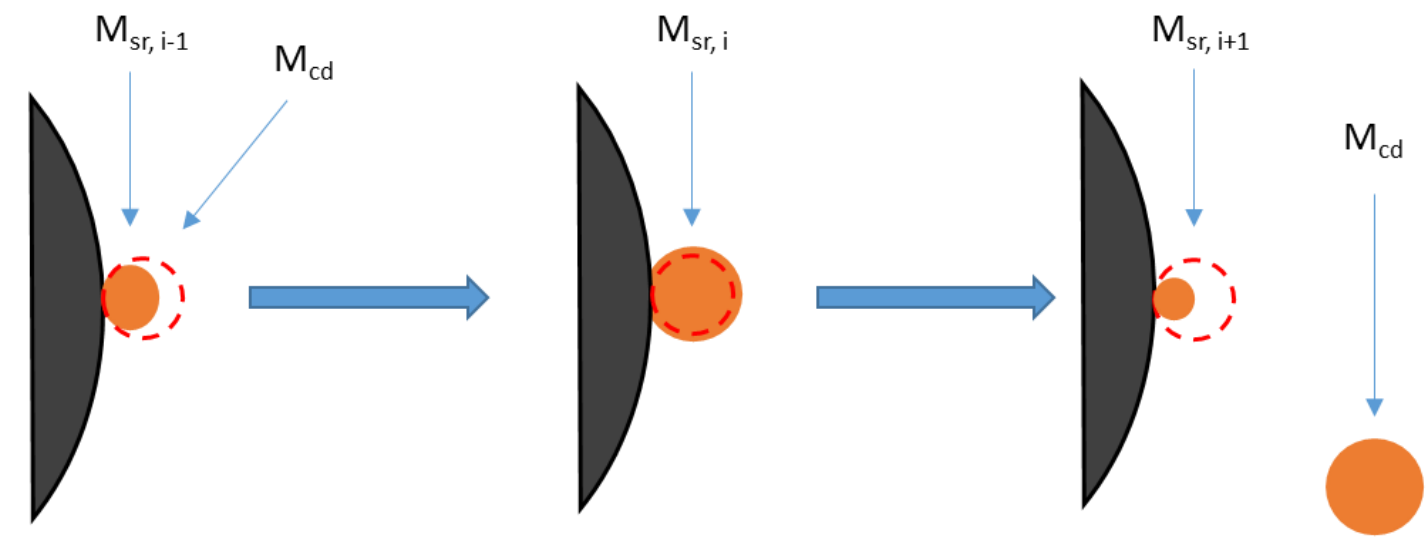

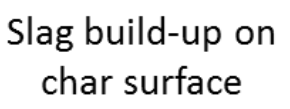

lag build-up on

char surface
Slag exceeds critical size in the control volume
Slag droplets separate into the bulk; remaining slag mass
left behind

Figure 3.6: Schematic for the slag droplet formation and detachment model

The char particle mass balance equation is given by Eq. 3.15.

$$
M_{c h, i}=M_{c h, i-1}-\Gamma_{s-g, i} V_{c h, i} \tau_{i}\left(1+\omega_{a s h}\right)
$$

The second term on the right hand side of Eq. 3.15 includes the loss of mass from the char particle due to heterogeneous reactions and due to slag formation. The heterogeneous reactions determine the rate at which the size of the char particle shrinks. The volume of the char particles is calculated from Eq. 3.16.

$$
M_{c h, i}=V_{c h, i} \rho_{c h}
$$


The density of the char particle, $\rho_{c h}$, in the slagging section of the gasifier is constant since a shrinking particle model is assumed (Maloney, 2005). At this point, the char particle is devoid of volatile matter and moisture that escape before the peak temperature is reached. The density of the char particle is calculated accordingly.

The overall mass balance for the char particles using both continuous and particle descriptions must be the same, as given by Eq. 3.17.

$$
N_{c h, i} V_{c h, i}=\left(1-\varepsilon_{i}\right)\left(1-\varepsilon_{s a, i}\right)\left(1-\varepsilon_{s d, i}\right)
$$

where, $V_{c h, i}$ represents the volume of a single char particle in the $i^{\text {th }} \mathrm{CV}$.

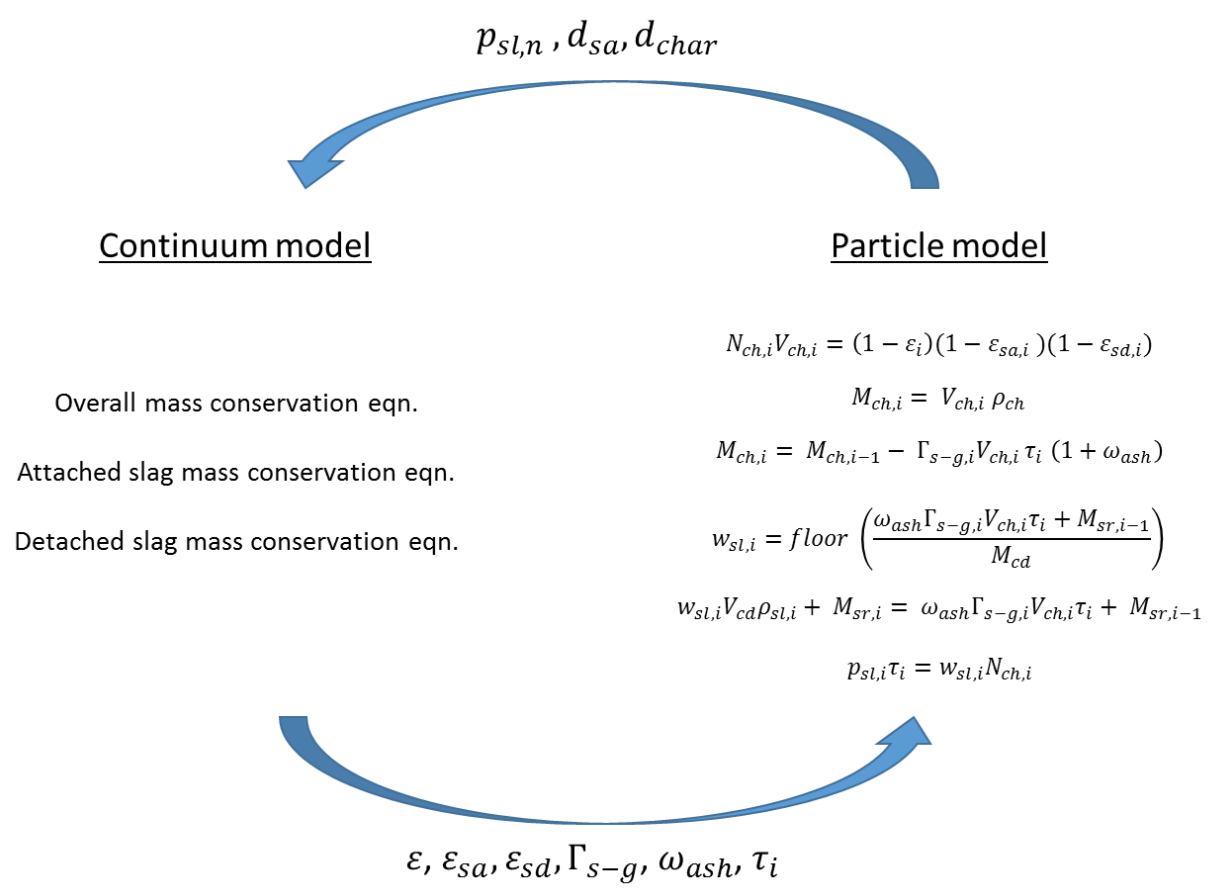

Figure 3.7: Schematic of information exchange between the continuum model and particle model

Figure 3.7 shows how the continuum model is coupled with the particle model. The coupling is done by number averaging with the assumption that the char particles are homogeneous in composition and equal in size in the same control volume. $w_{s l, i}$ as defined in Eq. 3.13 is an integer variable and therefore, a number averaged variable $p_{s l, i}$ is calculated from it using Eq. 3.11 and used in Eq. 3.9 and 3.10 for the continuum model. In addition, the volume fractions used in the continuum model are related to the particle model by Eq. 3.17. 


\subsubsection{Momentum Balance Equation}

It is assumed that the velocities of the slag droplets, both attached and detached, are the same as the char particle since the system is found to be dilute in terms of solid concentration and the difference in gas and solid velocities are found to be negligible. This assumption has been made mainly for simplicity and ensures the computational expense remains tractable for a dynamic model. But certainly, the model can be easily enhanced by relaxing this assumption. Under this assumption, momentum balances are required only for the gas phase and the overall solid phase and these balances are shown in Eqs. 3.18 and 3.19, respectively.

$$
\begin{gathered}
\frac{d\left(\varepsilon \rho_{g} U_{g}^{2}\right)}{d x}=-\varepsilon \frac{d P_{t}}{d x}+\varepsilon \rho_{g} g-(1-\varepsilon) f_{s} \\
\frac{d\left((1-\varepsilon) \rho_{s, a v g} U_{s}^{2}\right)}{d x}=-(1-\varepsilon) \frac{d P_{t}}{d x}+(1-\varepsilon) \rho_{s, a v g} g+(1-\varepsilon) f_{s}
\end{gathered}
$$

where, $f_{s}$ is the drag force per unit volume of particles, $U_{s}$ and $U_{g}$ are the solid and gas phase velocities respectively, $P_{t}$ is the total pressure in the system. The drag force is calculated using the equation from Arastroopour and Gidaspow (1979) as;

$$
f_{S}=\frac{3 C_{D} \rho_{g}(1-\varepsilon)^{-2.65}\left(U_{g}-U_{S}\right)\left|U_{g}-U_{S}\right|}{4 d_{\text {avg }}}
$$

where the $C_{D}$ is the drag coefficient taken from Rowe and Henwood (1961). This is given as

$$
C_{D}= \begin{cases}\frac{24}{R e}\left[1+0.15 R e^{0.687}\right] & ; R e<1000 \\ 0.44 ; & R e \geq 1000\end{cases}
$$

The Reynolds number is given as

$$
R e=(1-\varepsilon) \rho_{g} d_{a v g} \frac{\left|U_{g}-U_{S}\right|}{\mu_{g}}
$$

where, $\mu_{g}$, is the viscosity of the gas phase and $d_{\text {avg }}$ is the weighted average diameter of the slag droplets and char particles, calculated on the basis of their respective volume fractions. 


\subsubsection{Energy Balance Equation}

The energy balance equations for the gas and solid phases are shown in Eqs. 3.23 and 3.24. The temperature of the slag droplets and the char particle are assumed to be equal. This is done mainly for simplicity and keeping the computational expense tractable for a dynamic model. The model can be easily enhanced by relaxing this assumption.

$$
\begin{aligned}
& \frac{\partial\left(\varepsilon \rho_{g} C_{p, g} T_{g}\right)}{\partial t}+\frac{\partial\left(U_{g} \varepsilon \rho_{g} C_{p, g} T_{g}\right)}{\partial x}=\frac{\pi D_{i}}{A_{R}}\left\{h_{w-g}\left[T_{w}-T_{g}\right]\right\}-(1-\varepsilon) \frac{6}{d_{c h a r}}\left\{e_{g} F_{g-s} \sigma\left[T_{g}^{4}-T_{s}^{4}\right]+\right. \\
& \left.h_{g-s}\left[T_{g}-T_{s}\right]\right\}+\sum_{j}^{\text {gas-phase }} \text { reactions } \varepsilon\left(-\Delta H_{r x n, j}\right) r_{j}-m_{r g} h_{r g}+m_{m g} h_{m g} \\
& \frac{\partial\left((1-\varepsilon) \rho_{s, a v g} C_{p, a v g} T_{S}\right)}{\partial t}+\frac{\partial\left(U_{S}(1-\varepsilon) \rho_{s, a v g} C_{p, a v g} T_{S}\right)}{\partial x}=\frac{\pi D_{i}}{A_{R}} F_{w-s}\left[T_{w}^{4}-T_{s}^{4}\right]+(1-\varepsilon) \frac{6}{d_{c h a r}}\left(e _ { g } F _ { g - s } \sigma \left[T_{g}^{4}-\right.\right. \\
& \left.\left.T_{s}^{4}\right]+h_{g-s}\left[T_{g}-T_{s}\right]\right)+\sum_{k}^{\text {solid-phase }} \text { reactions }(1-\varepsilon)\left(-\Delta H_{r x n, k}\right) r_{k}
\end{aligned}
$$

where $F_{g-s}$ and $F_{w-s}$ are the view factors between gas-solid and wall- solid, respectively. In the gas phase energy balance equation, $m_{r g} h_{r g}$ is the enthalpy leaving and $m_{m g} h_{m g}$ is the enthalpy entering the control volume due to recirculation. $\Delta H_{r x n, j}$ and $\Delta H_{r x n, k}$ are the heat of reaction for the homogeneous and heterogeneous reactions, respectively. The heats of reactions and kinetic parameters have been taken from the literature cited in Kasule et al (2012). The authors could not find the heat of fusion for the ash in Illinois \#6 coal, however, based on the limited literature; it seems that the heat of fusion for ash in coal (Fulton, 1910; Niessen, 2010) is usually very small in comparison to the heat of reaction of the heterogeneous reactions. Therefore, the heat of fusion is not explicitly considered in this model. Furthermore, ash transformation reactions are not considered separately, but are assumed to take place spontaneously along with the char conversion reactions. Due to this assumption, the latent heat of fusion for ash can be readily included in the energy balance equations by modifying the heat of reaction for the heterogeneous reactions. In the solid phase energy balance equation, $C_{p, a v g}$ is the average specific heat calculated using the weighted average of the voidage fractions of char, slag droplets attached and slag droplets in the bulk. Eqs. 3.25-3.26 show how $h_{r g}$ and $h_{m g}$ are calculated.

$$
h_{r g}=\sum_{i=}^{N} y_{i} \int_{298}^{T} C_{p, i} d T
$$




$$
h_{m g}=\frac{1}{m} \sum_{k=1}^{r} h_{r g, k}
$$

where $N$ is the number of components in the gas phase, $r$ is the number of control volumes in the recirculation zone and $m$ is the number of control volumes in the mixing zone.

Eqs. 3.27-3.29 are used for the calculation of the average density and specific heat that is used in the momentum and energy balance equation for the solid phase.

$$
\begin{gathered}
\rho_{s, a v g}=\varepsilon_{s d} \rho_{s l}+\left(1-\varepsilon_{s d}\right) \varepsilon_{s a} \rho_{s l}+\left(1-\varepsilon_{s d}\right)\left(1-\varepsilon_{s a}\right) \rho_{c h} \\
d_{a v g}=\varepsilon_{s d} d_{c r}+\left(1-\varepsilon_{s d}\right) \varepsilon_{s a} d_{s a}+\left(1-\varepsilon_{s d}\right)\left(1-\varepsilon_{s a}\right) d_{c h} \\
\rho_{s, a v g} C_{p, a v g}=\varepsilon_{s d} \rho_{s l} C_{p, s l a g}+\left(1-\varepsilon_{s d}\right) \varepsilon_{s a} \rho_{s l} C_{p, s l a g}+\left(1-\varepsilon_{s d}\right)\left(1-\varepsilon_{s a}\right) \rho_{c h} C_{p, c h}
\end{gathered}
$$

The wall energy balance is taken from Kasule et al. (2012), and includes radiation between the wall and solids, radiation between wall and the top and bottom of the gasifier respectively, convection between wall and gas and the energy loss to the surrounding environment.

$\rho_{\text {wall }} C_{p, \text { wall }} \frac{\left(D_{o}^{2}-D_{i}^{2}\right)}{4} \frac{d\left(T_{w}\right)}{d x}=$

$\left(\pi D_{i} \Delta x\right) \sum_{i}\left(q_{\text {conv }, w-g}+q_{\text {rad }, w-g}+q_{\text {cond }, w-s k i n}+q_{\text {rad }, w_{i}-q_{\text {all }, p \neq l}}+q_{\text {rad, } w-t o p}+q_{\text {rad }, w-b t m}\right)$

where,

$$
\begin{aligned}
& q_{\text {conv }, w-g}=h_{w-g}\left(T_{w}-T_{g}\right) \\
& q_{\text {cond }, w-s k i n}=k_{e f f, w} \frac{\left(T_{w}-T_{\text {skin }}\right)}{\left(\left(D_{0}^{2}-D_{i}^{2}\right) / 4\right)} \\
& q_{\text {rad }, w-s}=F_{w-s} \sigma\left(T_{w}^{4}-T_{s}^{4}\right) \\
& q_{\text {rad }, w_{i}-w_{\text {all } \neq l}}=\sum_{p} e_{w} F_{w_{i}-w_{p}}\left(T_{w_{i}}^{4}-T_{w_{p}}^{4}\right)
\end{aligned}
$$




\subsubsection{Reaction Rates}

The gasifier can be divided into several reaction zones based on the dominant reactions/processes that occur in the solids. These reactions/ processes include drying, devolatilization, combustion, and gasification. The first three of these processes tend to occur much earlier in the gasifier, and result in a dramatic increase in the solid temperature. Gasification reactions are slower and continue till the end of the gasifier. In both shrinking core and shrinking particle models, all reactions are considered at all locations. The homogeneous reactions initiate soon after the devolatilization zone. The water gas shift reaction is one of the important reactions that takes place and determines the $\mathrm{CO} / \mathrm{H}_{2} \mathrm{O}$ ratio of the outlet syngas.

\subsubsection{Heterogeneous Reaction Kinetics}

Water vapor evaporation, devolatilization and the homogeneous reactions are modeled in the same manner as shown in Kasule et al (2012). Water evaporation is modeled similar to the work of Rao et al. (2007). A point to note is that the water in the slurry and the moisture content is considered together in calculation of the evaporation rate. For devolatilization, the products and kinetic parameters for the reaction / processes given by Syamlal and Bisset (1992) are used in the

model. The heterogeneous reaction rates are taken from the work of Wen and Chang (1979) and are summarized in the Table 3.1 . 
Table 3.1: Kinetic parameters of the heterogeneous reactions in the gasifier

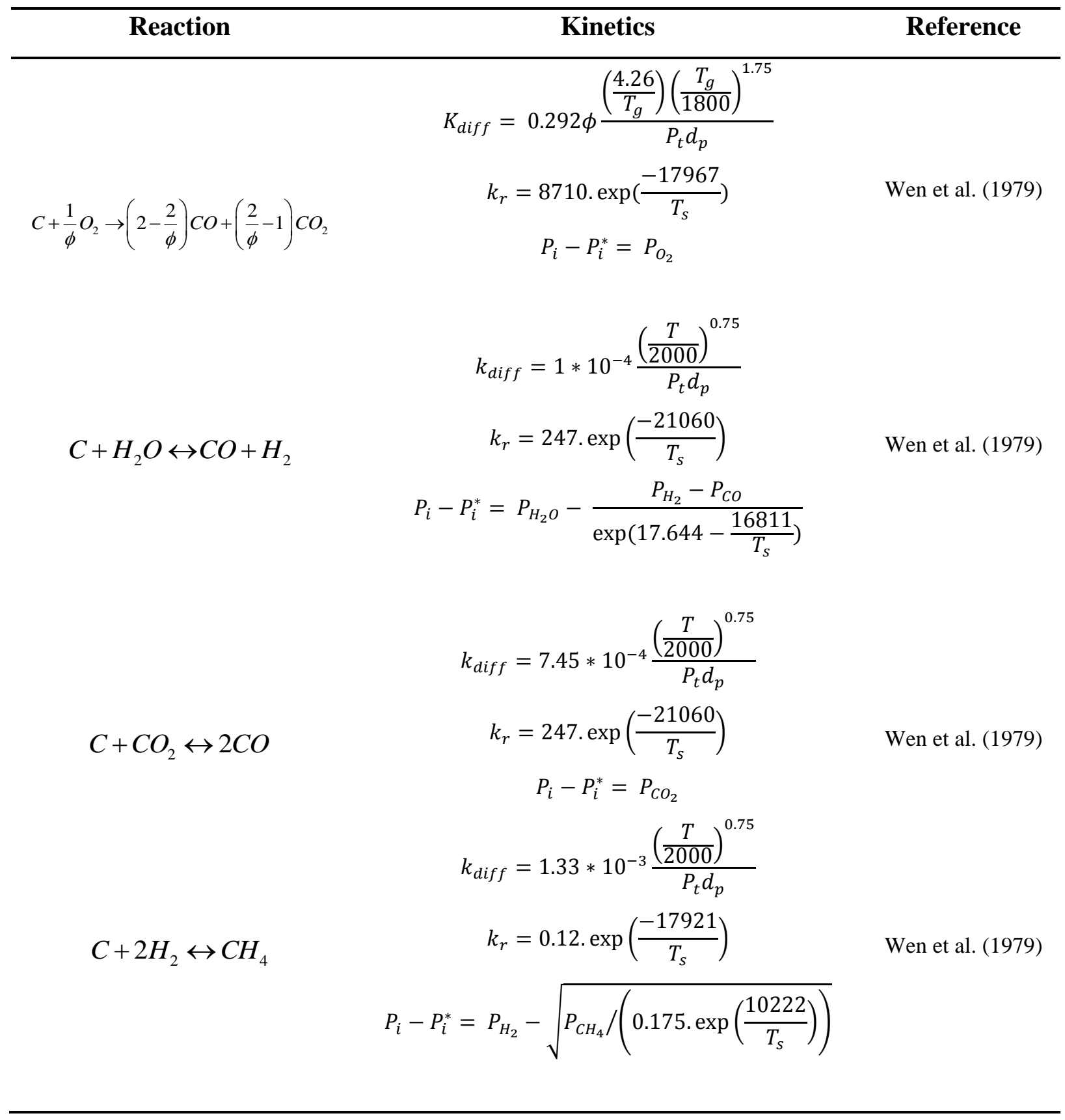


In the shrinking core section of the gasifier model, the overall reaction rate is calculated using Eq. 3.34 .

$$
K_{\text {overall }}=\frac{1}{\frac{1}{k_{\text {diff }}}+\frac{1}{k_{\text {ash }}}\left(1-\frac{1}{Y}\right)+\frac{1}{k_{S} Y^{2}}}
$$

where $Y$ is the ratio of the diameters of unreacted core and the char particle, and $k_{\text {diff }}, k_{\text {ash }}$ and $k_{S}$ are the gas film diffusion coefficient, ash diffusion coefficient and surface reaction coefficient respectively.

The overall reaction rate in the shrinking particle model is given as Eq. 3.35. The ash resistance in the shrinking particle model is assumed to be zero.

$$
K_{\text {overall }}=\frac{1}{\frac{1}{k_{\text {diff }}}+\frac{1}{k_{S}}}
$$

It should be noted that all rate constants are in the units of $\mathrm{g} \cdot \mathrm{cm}^{-2} \cdot \mathrm{atm}^{-1} \mathrm{~s}^{-1}$. Typically, a conversion factor of $6 / d_{\text {char }}$ is used to give the overall reaction rate constant in terms of volumetric units. For the present model, the surface reaction rate constant term cannot be evaluated at a shrinking particle size since the reaction rate would tend to infinity as the diameter of the char particle shrinks to zero. The surface reaction rate constant is instead converted to volumetric units by evaluating the factor $6 / d_{\text {char }}$ at the fixed char particle size. The particle size used by Wen and Chaung (1979) had considered while developing these kinetics was $350 \mu \mathrm{m}$. 


\subsubsection{Homogeneous Reaction Kinetics}

The homogeneous reactions, the kinetic parameters and the sources of the kinetics are listed in Table 3.2.

Table 3.2: Kinetic parameters of the homogeneous reactions in the gasifier

\begin{tabular}{|c|c|c|}
\hline Reaction & Rate of reaction & Reference \\
\hline $\mathrm{CO}+\frac{1}{2} \mathrm{O}_{2} \stackrel{k_{4}}{\longrightarrow} \mathrm{CO}_{2}$ & $3.98 \mathrm{e} 14 \cdot \exp \left(-40000 / \mathrm{RT}_{g}\right) \varepsilon C_{O_{2}}^{0.25} C_{C O} C_{H_{2} \mathrm{O}}^{0.5}$ & $\begin{array}{l}\text { Westbrook } \\
\text { et al. (1981) }\end{array}$ \\
\hline $\mathrm{CH}_{4}+2 \mathrm{O}_{2} \stackrel{k_{5}}{\longrightarrow} \mathrm{CO}_{2}+2 \mathrm{H}_{2} \mathrm{O}$ & 6.7e12. $\exp \left(-\frac{48400}{R T_{g}}\right) \varepsilon C_{O_{2}}^{1.3} C_{C H_{4}}$ & $\begin{array}{l}\text { Westbrook } \\
\text { et al. (1981) }\end{array}$ \\
\hline $\mathrm{H}_{2}+\frac{1}{2} \mathrm{O}_{2} \stackrel{k_{6}}{\longrightarrow} \mathrm{H}_{2} \mathrm{O}$ & 1.08e6. $\exp \left(-30000 / R T_{g}\right) \varepsilon C_{O_{2}} C_{H_{2}}$ & $\begin{array}{l}\text { Peters } \\
(1979)\end{array}$ \\
\hline $\mathrm{CO}+\mathrm{H}_{2} \mathrm{O} \stackrel{k e q_{1}}{\longleftrightarrow} \mathrm{CO}_{2}+\mathrm{H}_{2}$ & 2.877e10. $w_{g 3} f_{3} P^{\left(0.5-\frac{P}{250}\right)} \cdot \exp \left(-\frac{27760}{R T_{g}}\right) \cdot\left(x_{c o} x_{H 2_{O}}-\frac{x_{\mathrm{CO}_{2}} x_{\mathrm{H}_{2}}}{K_{e q}}\right)$ & $\begin{array}{l}\text { Kasule et al. } \\
\quad(2012)\end{array}$ \\
\hline $\mathrm{CH}_{4}+\mathrm{H}_{2} \mathrm{O} \stackrel{\mathrm{keq}_{2}}{\longleftrightarrow} \mathrm{CO}+3 \mathrm{H}_{2}$ & $k_{f}=3 e 11, E_{a, f}=15105 ; k_{b}=5 e-14, E_{a, b}=27300$ & $\begin{array}{l}\text { Wen et al. } \\
\text { (1979) }\end{array}$ \\
\hline$\frac{1}{2} \mathrm{~N}_{2}+\frac{3}{2} \mathrm{H}_{2} \stackrel{k e q_{3}}{\longleftrightarrow} \mathrm{NH}_{3}$ & $k_{f}=1053, E_{a, f}=5970 ; k_{b}=46607, E_{a, b}=11225$ & $\begin{array}{l}\text { Friedrichs et } \\
\text { al. (2000) }\end{array}$ \\
\hline
\end{tabular}

The kinetics for the water-gas shift reaction (WGS) was modeled as a combination of a catalytic rate (Wen et al., 1982) and a modified form of the non-catalytic rate (Karan et al., 1999) and is taken from Kasule et al. (2012) 


\subsection{Model inputs}

The model for the slagging gasifier has been developed in Aspen Custom Modeler ${ }^{\circledR}$ (ACM). The system of partial differential equations and algebraic (PDAE system) are solved simultaneously using a backward finite difference method. Table 3.3 lists model parameters and input conditions. Table 3.4 provides the proximate analysis of Illinois \#6 coal used in this study.

Table 3.3: Model parameters and input conditions

\begin{tabular}{cc}
\hline Parameters/Conditions & Value \\
\hline Gasifier parameters & 662 \\
Length $(\mathrm{cm})$ & 179 \\
Internal diameter $(\mathrm{cm})$ &
\end{tabular}

\section{Operating conditions}

\begin{tabular}{cc} 
Coal slurry flow rate $(\mathrm{g} / \mathrm{s})$ & 61232.9 \\
Particle diameter $(\mu \mathrm{m})$ & 100 \\
Water to coal ratio & 0.4115 \\
$\mathrm{O}_{2}$ to coal ratio & 0.8347 \\
Inlet Temperature $\left({ }^{\circ} \mathrm{C}\right)$ & 29.85 \\
Inlet Pressure $($ bar $)$ & 28.33 \\
\hline
\end{tabular}

Table 3.4: Proximate and Ultimate analysis of Illinois \#6 coal

\begin{tabular}{lc}
\hline \multicolumn{1}{c}{ Illinois \#6 } & As-Received (wt \%) \\
\hline Proximate analysis & \\
Fixed Carbon & 44.19 \\
Ash & 9.99 \\
Volatile matter & 34.70 \\
Moisture & 11.12 \\
& \\
Ultimate analysis (DAF) & \\
C & 63.75 \\
H & 4.50 \\
O & 6.88 \\
N & 1.25 \\
S & 2.51 \\
\hline
\end{tabular}




\subsection{Results}

The results from the HSCSP model are summarized below. These include the validation of the data as compared to the TECO power plant (TAMPA Final Technical Report, 2002), comparison with the traditional shrinking-core model, profiles of key variables and a sensitivity analysis on the detachment diameter.

\subsubsection{Model Validation}

In this section, the results were obtained assuming complete coalescence of slag droplets, which should closely resemble the results from the shrinking-core model assuming no slag detachment. This is compared first with the industrial data of TECO power plant. The gasifier configuration of the TECO power plant and the operating conditions are shown in Table 3.5.

Table 3.5: Validation data from TECO power plant

\begin{tabular}{lc}
\hline \multicolumn{1}{c}{ Conditions } & TECO \\
\hline Gasifier configuration & 179 \\
Internal diameter $(\mathrm{cm})$ & 662 \\
Length $(\mathrm{cm})$ & \\
& \\
Operating conditions & 40 \\
Coal feed rate $(\mathrm{kg} / \mathrm{s})$ & 100 \\
Coal particle size $(\mu \mathrm{m})$ & 0.82806 \\
Oxygen/coal ratio & 0.4108 \\
Water/coal ratio & 26 \\
Pressure $($ atm $)$ & \\
\end{tabular}

The data from the TECO power plant are available for the clean syngas that is downstream of the radiant syngas cooler (RSC). In the RSC, steam is produced by utilizing the energy in the gasifier exit stream. It has been reported that certain gas-phase reactions, such as the water-gas shift 
reaction, continue to take place in the initial section of the RSC (Kasule et al., 2012). Therefore, for comparing the results with the TECO Power plant, a simple model of the RSC was developed in Aspen Plus.

The RSC is modeled using a plug flow reactor. This model is implemented in a similar manner as done in the work of Kasule (2012). A constant cooler temperature of $609 \mathrm{~K}$ was assumed.

Figure 3.8 shows that the results from the HSCSP model have a good qualitative agreement with the TECO data.

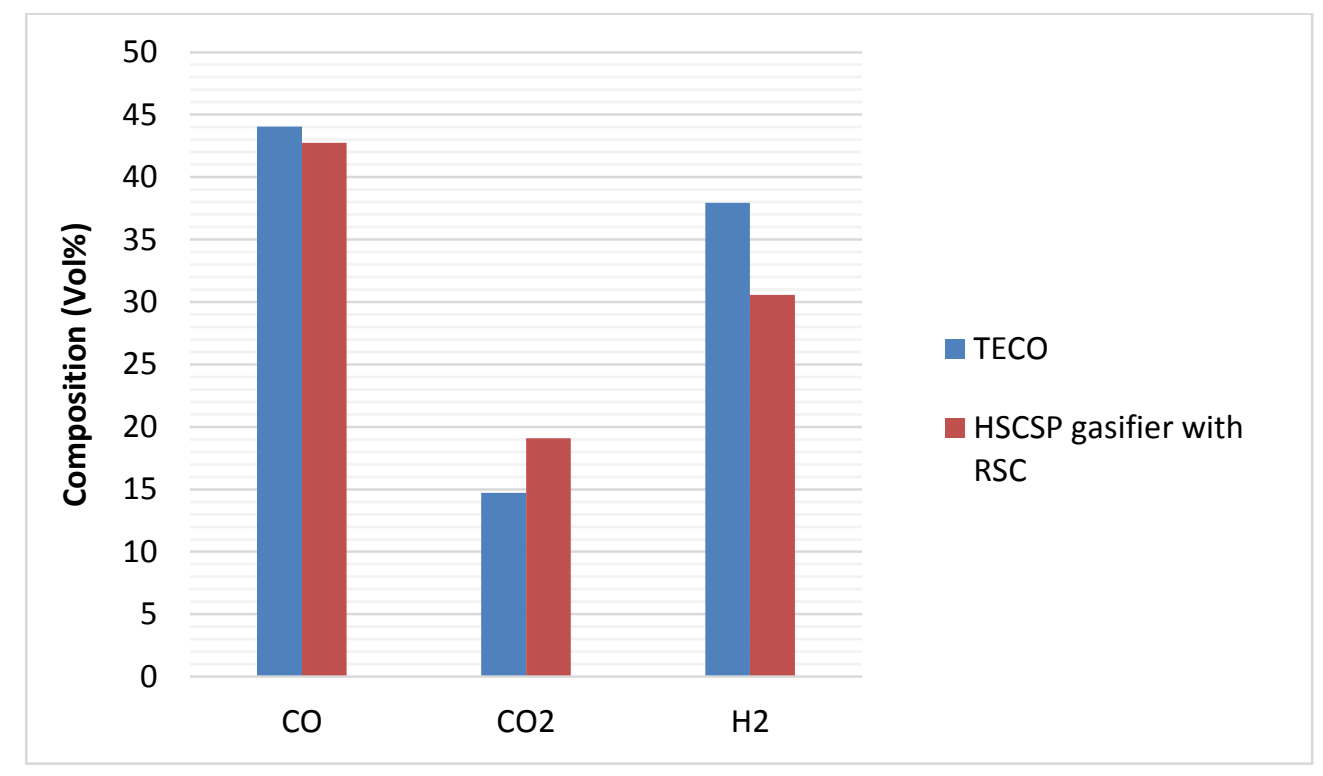

Figure 3.8: Comparisons of the mole fractions of $\mathrm{CO}_{2}, \mathrm{CO}, \mathrm{H}_{2}$ and $\mathrm{H}_{2} \mathrm{O}$ (on dry basis) at the exit of the RSC with TECO data 


\subsubsection{Shrinking Core vs HSCSP Model}

The results from this work are compared with the shrinking-core model developed by Kasule et al. (2012). For a fair comparison, feed composition, flow rates, pressures, and $\mathrm{O}_{2} /$ Coal ratio are set to be the same in both the models.

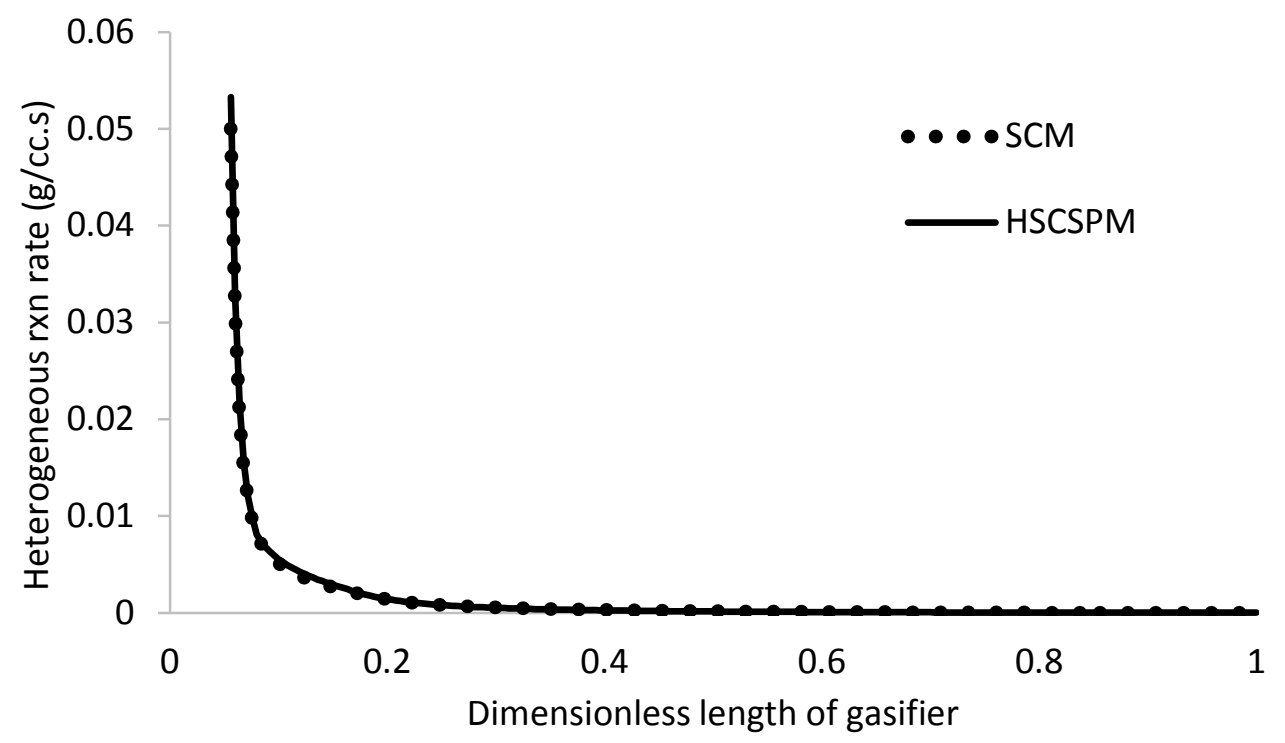

Figure 3.9: Comparison of the reaction rates between the shrinking particle model and hybrid shrinking core-shrinking particle model

Figure 3.9 shows the heterogeneous reaction rates for both the models after combustion of char takes place, i.e., in the region where the SPM is applied. In the SCM, the overall reaction rate is limited by the resistance due to the ash layer which is zero for the SPM model. Furthermore, the diffusion resistance of a shrinking particle would be lower than that calculated in the SCM. However, the volume of the particle keeps decreasing in the SPM. Overall, there is hardly any difference in the heterogeneous reaction rate as shown in Figure 3.9. 


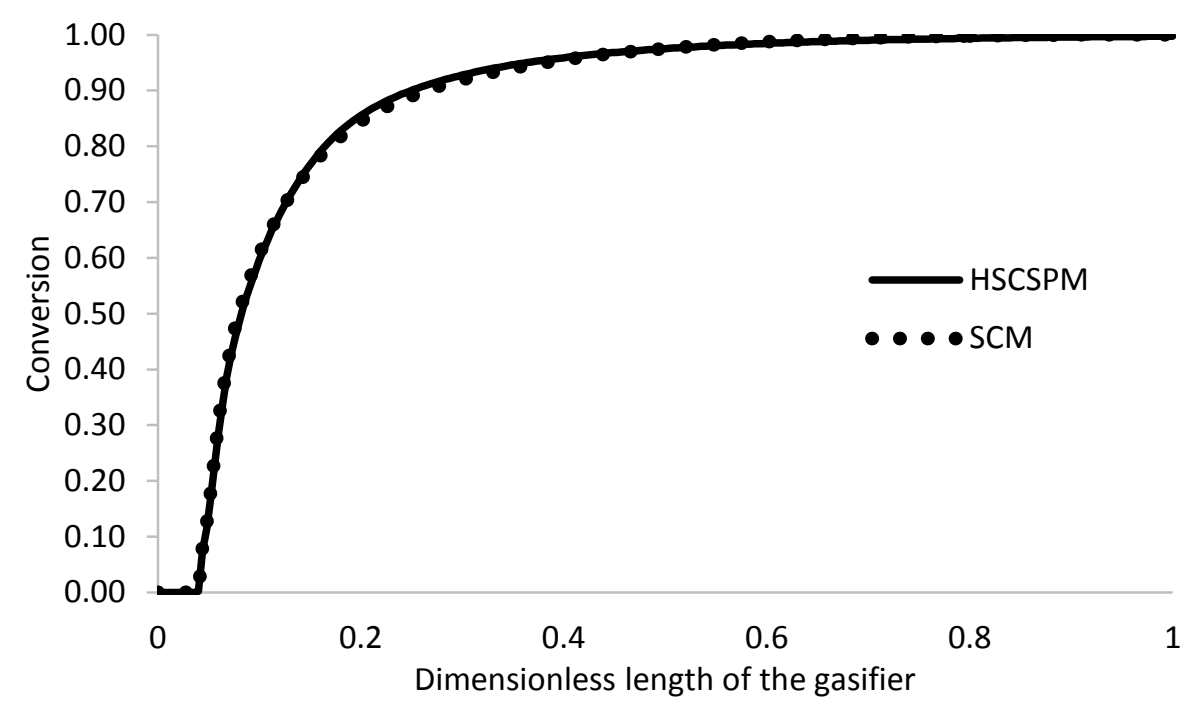

Figure 3.10: Comparison of carbon conversion of carbon between the gasifier model and the complete coalescence model

Figure 3.10 compares carbon conversion obtained in this work to that obtained using the SCM. In both the cases, a significant amount of carbon gets converted very early in the reactor followed by slower conversion, which is mainly due to the gasification reactions.

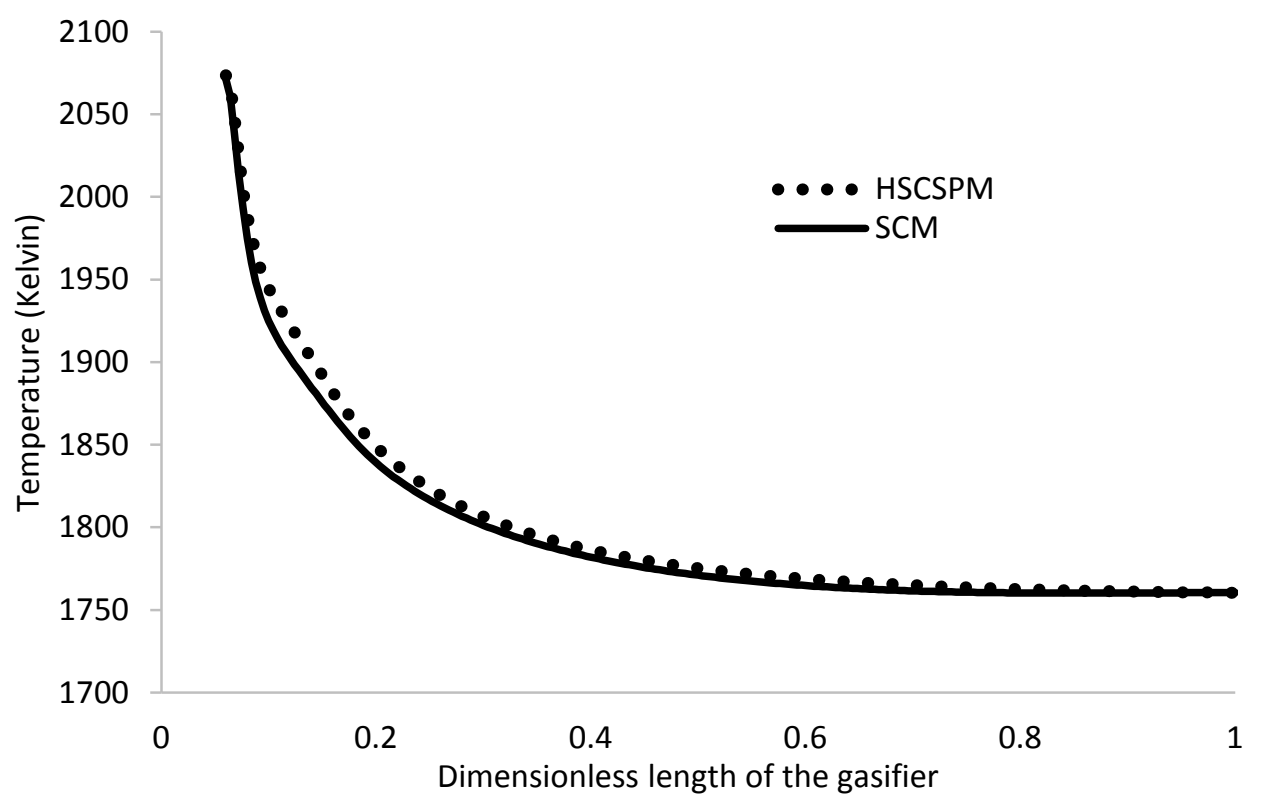

Figure 3.11: Comparison of solids temperature profiles between the shrinking core and HSCSP model 
Figure 3.11 compares the temperature of the char particle using the SCM with the HSCSP model developed in this work. As mentioned earlier, in the initial region of the gasifier up to the point when the bulk temperature exceeds the ash melting temperature the HSCSP model considers shrinking core assumption and therefore, the solid and gas temperatures closely match that from the SCM in this region. Therefore in Figure 3.11, the solids temperature profile beyond this initial region is compared. The solids temperature from the HSCSP model is found to be little higher towards the beginning of this section. However, towards the end, both models reach similar conversion and the exit temperatures are the same. The gas temperature also follows the same trend (not shown here).

Comparing the mole fractions at the exit of the gasifier for the SCM and HSCSP models in Table 3.6, we see that there is very little difference between the two models.

Table 3.6: Comparison between outlet mole fractions of SCM and HSCSP models

\begin{tabular}{lcc}
\hline Component & SCM & HSCSPM \\
\hline $\mathrm{CO}_{2}$ & 0.22396 & 0.22531 \\
$\mathrm{CO}$ & 0.47236 & 0.47052 \\
$\mathrm{H}_{2}$ & 0.01848 & 0.01842 \\
$\mathrm{H}_{2} \mathrm{O}$ & 0.20823 & 0.20883 \\
\hline
\end{tabular}




\subsubsection{Complete Coalescence Case}

In this scenario, it is assumed that the slag droplets are not detached from the char particles. Figure 3.12 shows that even though the char conversion is high, the char particle still exits at some finite size that exits the gasifier. The slag droplet attached to the char particle grows rapidly initially when the conversion is high. It begins to level off towards the end due to the decrease in conversion rate.

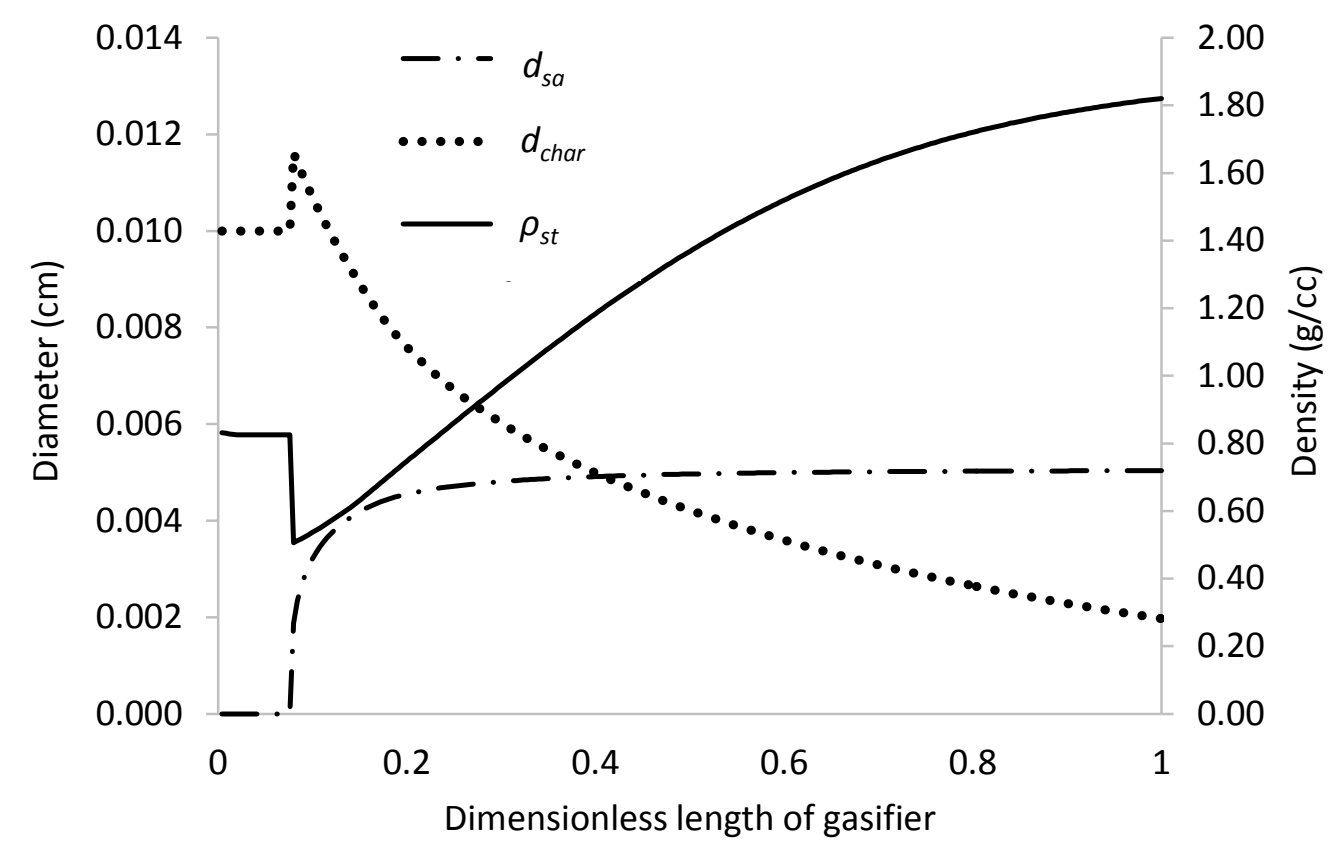

Figure 3.12: Variation of diameter of char particles, attached slag droplets, and average density of the char-slag system along the gasifier

Figure 3.12 shows the profiles for the diameters and densities in the zone where the SPM is applied, i.e., after the bulk temperature increases beyond the ash melting temperature. Figure 3.12 also shows that the average density calculated using Eq. 3.27 keeps increasing along the gasifier as char content continues to decrease while slag content keeps increasing, which is because the slag droplets have higher density than the char particles. In contrast, in the SCM, the density keeps decreasing as mass disappears while the volume of the char particle remains constant. 


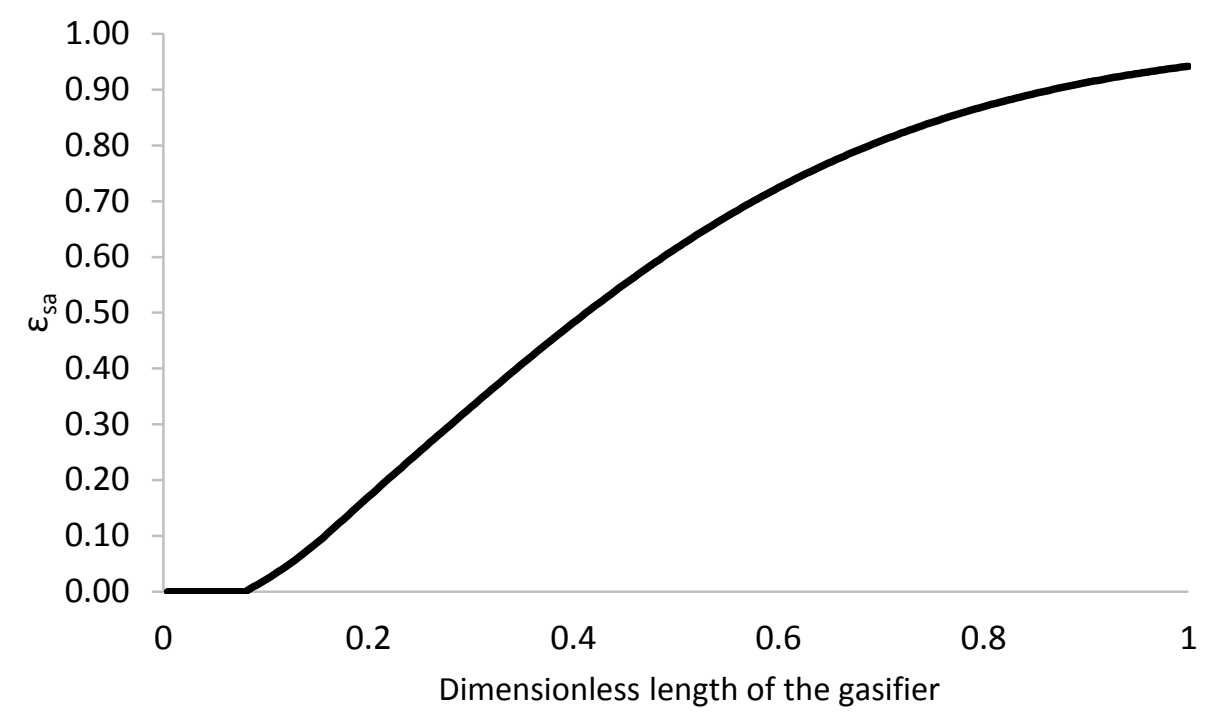

Figure 3.13: Variation of $\varepsilon_{\mathrm{sa}}$ along the gasifier for complete coalescence case

Figure 3.13 shows the profile of $\varepsilon_{\mathrm{sa}}$ along the gasifier length. As slag builds up on the char particle, $\varepsilon_{\mathrm{sa}}$ keeps increasing. However, it should be noted that it does not reach a value of unity since the volume of the unreacted char particle is finite at the end of the gasifier. 


\subsubsection{Slag Formation and Detachment Results}

\section{Key results from slag detachment scenario}

In this work, three discrete detachment diameters, 5, 10 and $15 \mu \mathrm{m}$, are considered for the slag droplets. Figure 3.14 shows the $\varepsilon_{\mathrm{sa}}$ profile along the gasifier. Unlike the complete coalescence scenario, where $\varepsilon_{\mathrm{sa}}$ keeps increasing monotonically, Figure 3.14 shows a saw tooth-type profile.

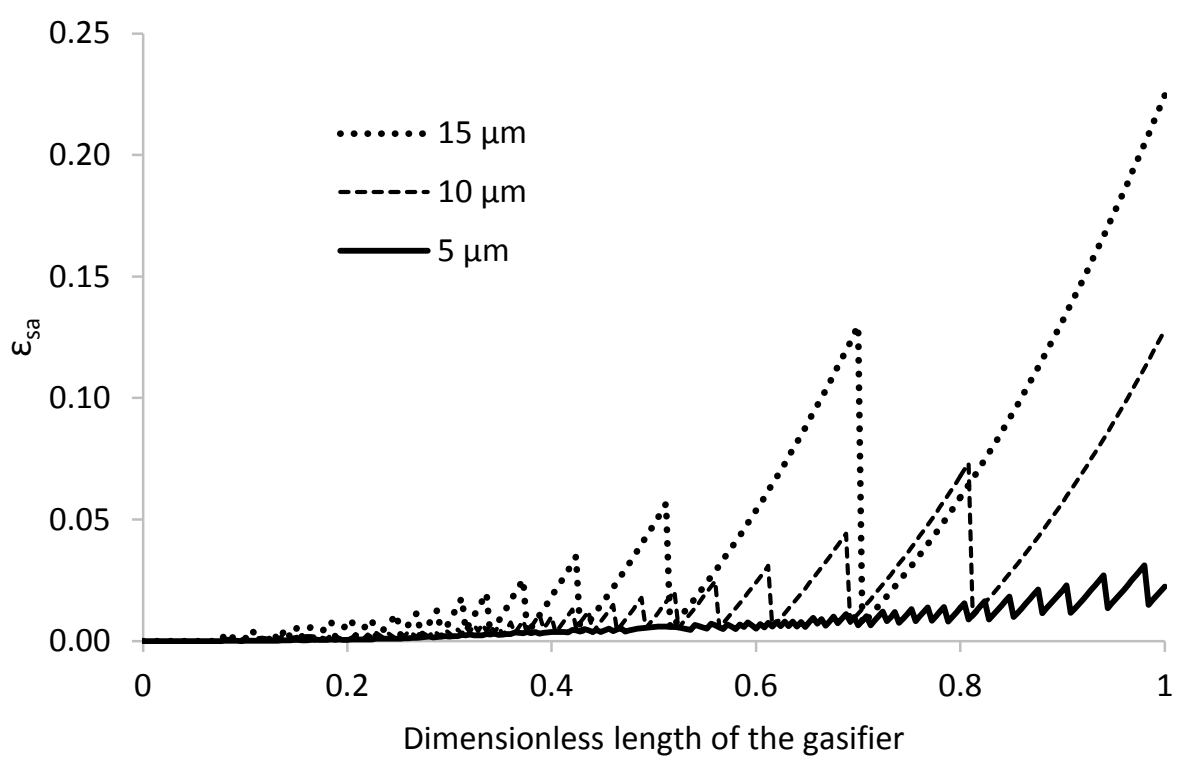

Figure 3.14: Variation of $\varepsilon_{\mathrm{sa}}$ along the gasifier for the slag detachment scenario

As expected, Figure 3.14 shows that the smaller the detachment diameter, the smaller is the build-up on the char particle's surface. The peaks begin to increase in height along the gasifier because the volume of the char particle shrinks as the char particle reacts. Therefore even though the detachment diameter remains the same for each case, the volume fraction of the slag droplet in comparison to char particle keeps increasing. It should be noted that in real life, it would be expected that the detachment diameter would change along the gasifier. The present study provides an idea of the expected range of variation for that case. 


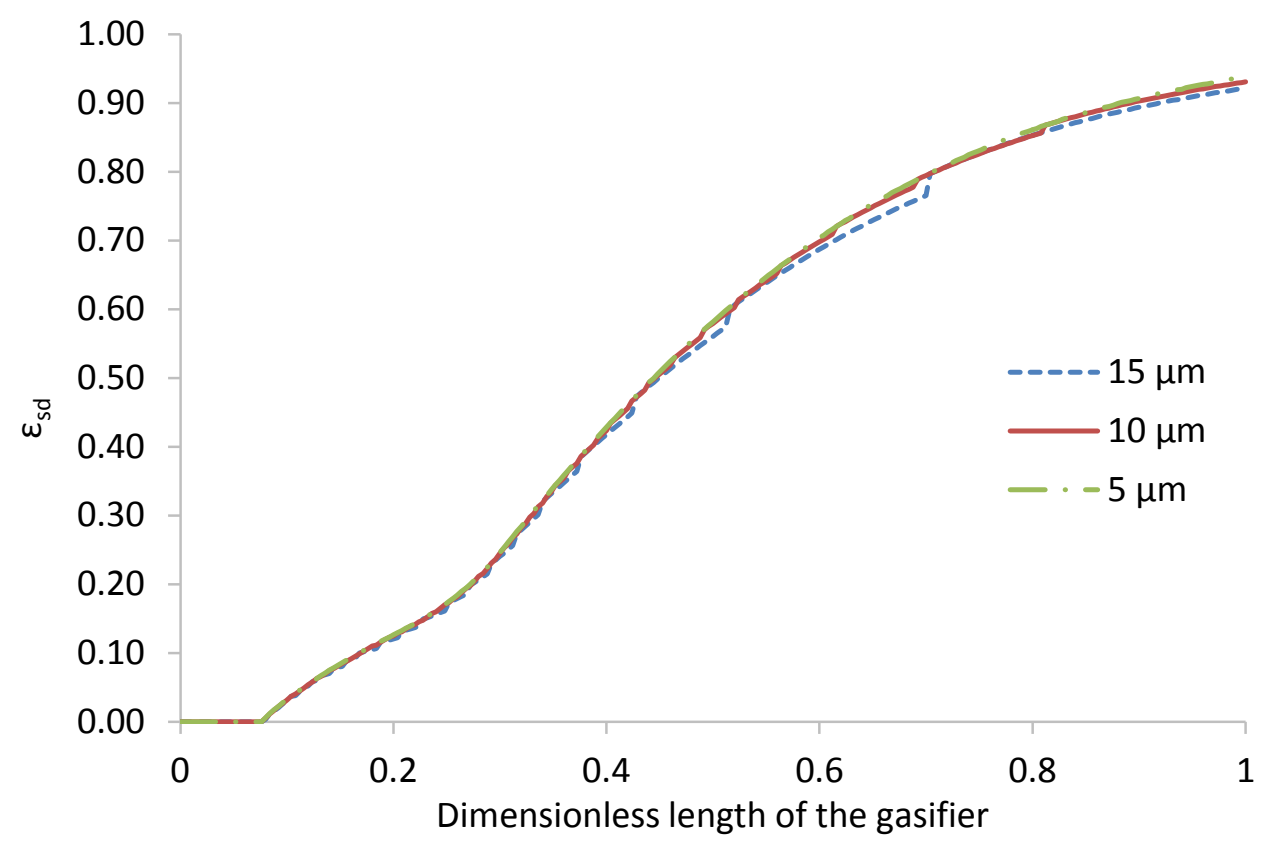

Figure 3.15: Variation of detached slag volume fraction along the gasifier

Figure 3.15 shows profile of $\varepsilon_{\mathrm{sd}}$, the volume fraction of the detached slag in the bulk of the gasifier. Even though the source term is a discontinuous variable, the profile of $\varepsilon_{\mathrm{sd}}$ is reasonably smooth, especially for smaller detachment diameters.

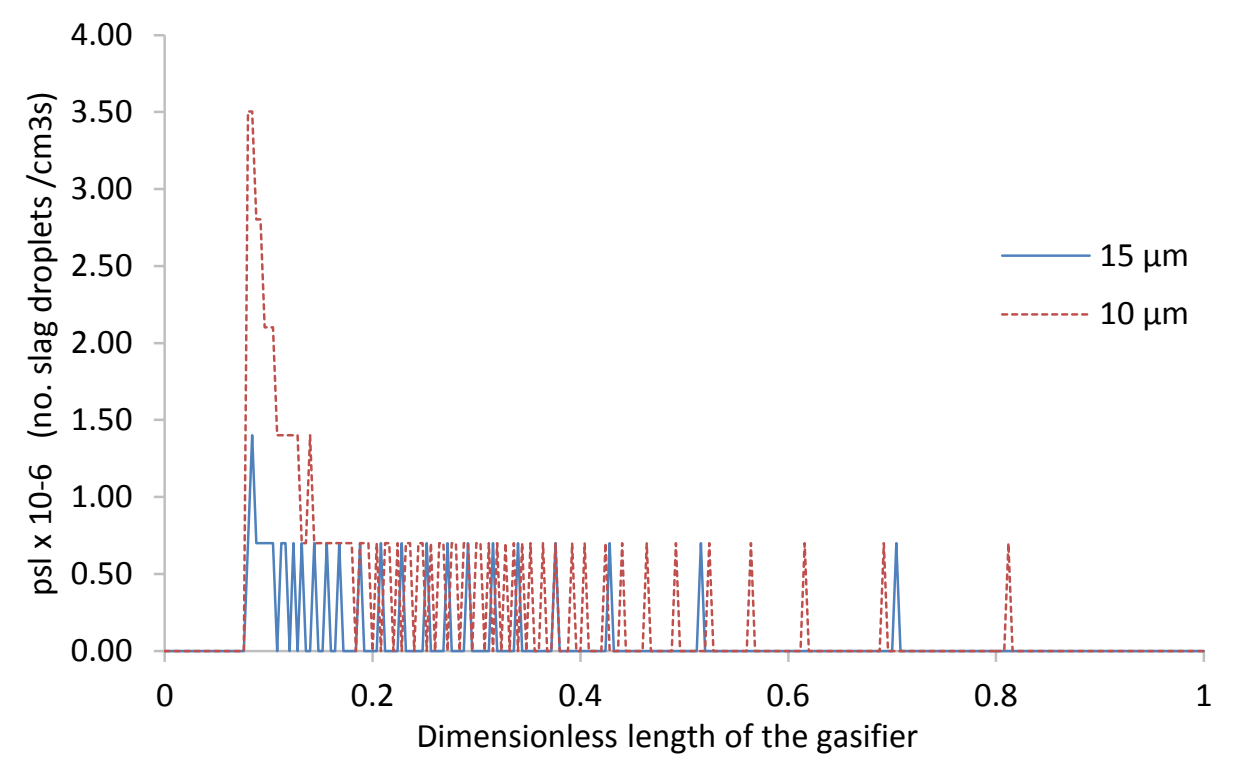

Figure 3.16: Variation of $\mathrm{p}_{\mathrm{sl}}$ along the gasifier for 10 and 15 micron case 
Figure 3.16 shows the profile of $p_{s l}$, i.e., number of slag droplets separated per unit volume per unit time for different slag detachment diameters. As expected, the smaller the detachment diameter, higher the value of $p_{s l}$. It can be seen that the number density of the slag droplets as well as their sizes significantly impacts the deposition flux to the gasifier wall.

\subsection{Conclusions}

A HSCSP model of the entrained-flow gasifier is presented. In this model, the shrinking core model is applied in the initial region of the gasifier while a novel shrinking particle model is developed for the later region. This model is more physically realistic than the traditional SCM, yet yields similar results. The SPM is developed by integrating a continuous model with a particle model. Carbon conversion and gasifier exit conditions obtained from the HSCSP model compare well with the industrial data. The model provides information about the particle density of char particles, fraction of slag droplets that are attached to the char particles, and fraction of slag droplets that are detached but exist in the bulk. In addition, the sizes of char particles and attached slag droplets can be tracked along the gasifier. It is also observed that even though the slag detachment is a discontinuous phenomenon, the profile of the volume fraction of detached slag remains reasonably smooth. The number of slag droplets separated per unit volume per unit time is found to increase considerably as the detachment diameter decreases. This information is very valuable for calculating the slag deposition rate on the gasifier wall. It should be noted that the thickness of the slag layer depends on the slag deposition rate on the gasifier wall and the slag layer thickness is a critical variable to ensure uninterrupted operation of the slagging gasifiers. These aspects will be investigated in the next chapter. 


\section{GASIFIER MODEL WITH SLAG DEPOSITION AND FLOW}

\subsection{Introduction}

Molten slag flows along the wall of slagging entrained-flow gasifiers. A lower operating temperature of these gasifiers can result in slag buildup that may eventually lead to shut down. Thus, the thickness of the slag layer is an important operating variable. However, it is difficult to monitor the thickness of the slag layer due to the extremely harsh environment inside these gasifiers. To investigate the impact of various operating conditions on the slag layer thickness, a dynamic model is required that can be is used to identify important variables that significantly affect the slag layer thickness. Optimal operational parameters can reduce the risk of slag solidification and to avoid excessive temperatures in the gasifier that could accelerate the refractory degradation process while maintaining a desired carbon conversion is also a priority. In this chapter, slag deposition and slag layer models have been developed and integrated into the novel shrinking-core shrinking-particle model of a downward-firing, entrained-flow, slurryfed, oxygen-blown gasifier, presented in Chapter 3. Slag deposition; due to char impaction and slag droplet impaction have been considered. The enhanced model is used to study the effect of particle size distribution, switch in coal type, change in ash composition and various operating conditions on the slag layer thickness. It is desired that the models should be reasonably accurate yet computationally tractable so that the dynamic model can be used for estimation and control studies. 


\subsection{Model Development}

Due to the exothermic homogeneous combustion reactions that immediately follow the devolatilization reactions, there is a sharp temperature gradient at the entrance of the gasifier. A high temperature is attained very quickly and the heterogeneous reactions readily take place resulting in slag formation (Pednekar et al., 2016). Since the char temperature remains above the ash melting temperature throughout the gasifier, it is likely that the ash present in the char particles melts forming slag droplets. Some of these slag droplets may detach from the char particles. Therefore, the traditional shrinking core model used in the literature, where the ash is considered to form a shell around the char particle, does not seem to be a good characterization. The authors recently presented a new hybrid shrinking-core shrinking-particle model (Pednekar et al., 2016), where a shrinking core model is applied until the solid temperature exceeds the melting point of the ash after which a shrinking particle model is applied. In the shrinking particle model, it was assumed that the slag droplets keep growing in size as char reacts. Eventually, the slag droplets reach a critical size and become detached. In our previous work, three cases were modeled where slag droplet sizes of 5,10 and $15 \mu \mathrm{m}$ were considered. 


\section{Gasifier bulk}

\section{Refractory layer}

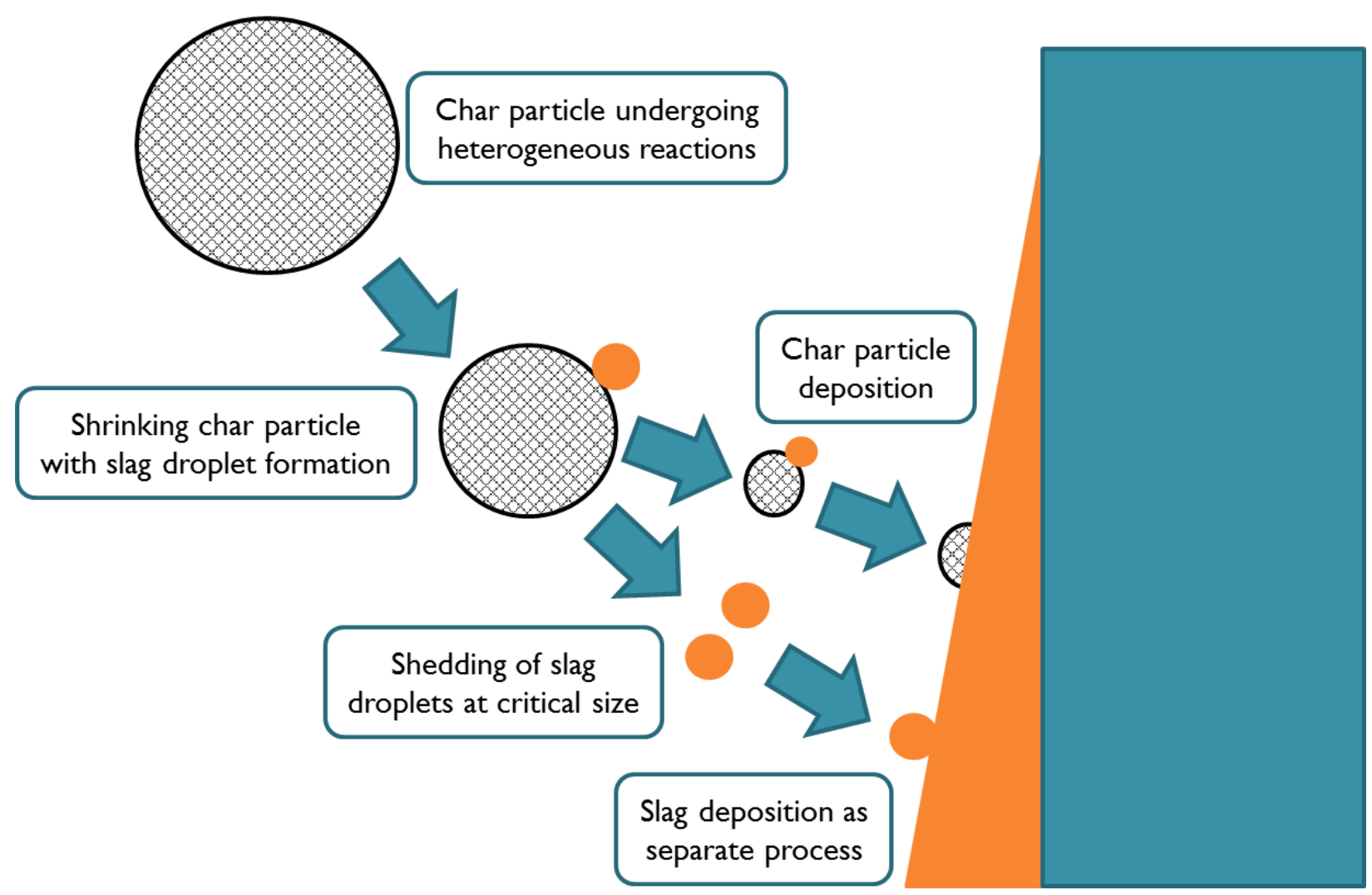

Figure 4.1: Schematic of the formation mechanism of slag droplets and their deposition on the wall along with char particles and the subsequent formation of a slag layer on the gasifier wall

In this chapter, a PSD is considered for the detached slag droplets. A model is developed to calculate the deposition flux for both char particles and slag droplets, both of which contribute to the slag flow along the wall that eventually leaves the gasifier from the bottom. A schematic of the proposed model is shown in Figure 4.1. 


\subsubsection{Assumptions}

The following assumptions have been made in developing the current model:

1. Char particles and slag droplets are spherical.

2. The constituents of the coal particle are assumed to be uniformly distributed.

3. Radial distribution of char particles and slag droplets is uniform.

4. No char or slag deposition is considered to take place in the region where the temperature of the solids is below the melting point of ash.

5. Excluded minerals, or minerals not associated with the char particles, are not considered in this study.

6. The capture efficiency for char and slag droplets at the wall is assumed to be unity when the solids temperature exceeds the melting point of ash. The assumption for the char particles has been made based on the observation of the results from the present model that when the char particles impact the wall, they already contain substantial amounts of slag. Therefore, it is likely that the impacting slag droplets will be fully captured.

7. Slag layer properties including thermal conductivity, specific heat, and density are assumed to be constant.

8. Due to the small thickness of the slag layer, a linear temperature profile is assumed in the slag layer.

9. The slag layer viscosity is assumed to be constant along the slag layer thickness.

10. The momentum equation for the slag layer is solved analytically and used in the model. 


\subsubsection{Particle Size Distribution}

Combustion or gasification conditions are often simulated using drop-tube furnaces. The ash resulting from these tests is segregated on the basis of its size and mass. A wide variation in PSD is observed depending on the coal type, coal particle size, gas flow rates, temperatures and other operating conditions in experimental work as discussed in Section 2.1.3. The mechanisms for the formation of droplets of different sizes differ and depending upon the conditions, some mechanisms may be dominant. Some tests show the presence of ash particles in the millimeter range which can form due to melting of larger excluded ash particles. In the present study, a PSD of slag droplets that form only due to the liberation of included slag droplets has been considered (Wu et al., 1999). The gas-solid system in the gasifier is assumed to be dilute and therefore the formation of large slag droplets due to the collision of two or more char particles is not considered. In the present model, slag droplets of the largest size can form when most or all of the ash initially present in the char particle separates as a single slag droplet. Smaller slag droplets of sizes between 1-10 microns can form due to liberation or shedding of included ash while partial coalescing of the included ash before separation would result in slag droplets with sizes between the two size ranges.

\subsubsection{Incorporating PSD into Mass Balance Equations}

A continuum model is developed for the solid and gas phases and integrated with a particle model developed for the char particles and slag droplets. Figure 4.2 shows the schematic representation of the notations used in this work. The volume fraction of the gas is denoted by $\varepsilon$. The volume fraction of the detached slag droplets is represented by $\varepsilon_{s d}$. The volume fraction of the attached slag droplets is denoted by $\varepsilon_{s a}$. A particle size distribution is considered, where the detached slag droplets are divided into four size bins depending on the diameters of the slag droplets: 1-10, 10-20, 20-30 and greater than $30 \mu \mathrm{m}$. The volume fractions of these bins in the bulk are denoted by $\varepsilon_{d, 1}, \varepsilon_{d, 2}, \varepsilon_{d, 3}$, and $\varepsilon_{d, 4}$, respectively. It should be noted that due to consideration of these four bins, modifications have been made to the equations presented in the earlier chapter and additional equations have been developed. 

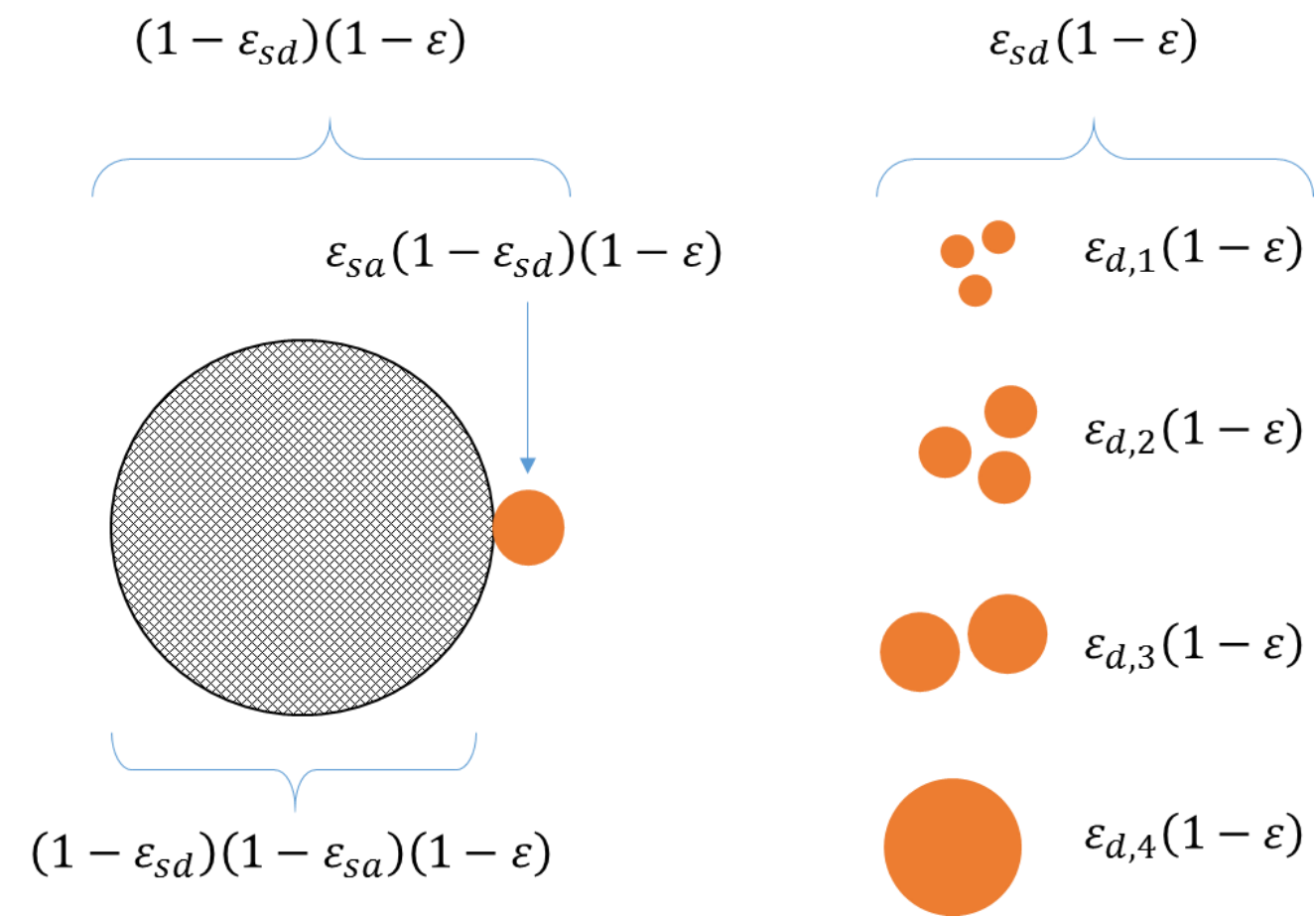

Figure 4.2: Schematic representation of the notations for denoting solids fractions in the continuum model

\subsubsection{Continuum Model}

The overall mass balance of the solid phase is modeled by Eq. 4.1. Solids are lost to the gas phase due to the heterogeneous reactions and due to the deposition of char particles and slag droplets onto the wall. These loss mechanisms are represented by the second and third terms on the right hand side of Eq. 4.1, respectively.

$$
\frac{\partial\left(\rho_{s, a v g}(1-\varepsilon)\right)}{\partial t}=-\frac{\partial\left(\rho_{s, a v g}(1-\varepsilon) U_{s}\right)}{\partial x}-(1-\varepsilon)\left(1-\varepsilon_{s a}\right)\left(1-\varepsilon_{s d}\right) \Gamma_{s-g}-\frac{4 m_{d e p}}{D_{i}}
$$

Here, $\rho_{s, a v g}$ is the average density of the solid phase comprising of the char particles and slag droplets, $U_{s}$ is the solid phase velocity, $\Gamma_{s-g}$ is the sum of all heterogeneous reaction rates, $m_{d e p}$ is the net deposition flux to the wall considering deposition of both char particles and slag droplets and $D_{i}$ is the internal diameter of the gasifier. The term $(1-\varepsilon)\left(1-\varepsilon_{s a}\right)(1-$ $\left.\varepsilon_{s d}\right)$ represents the volume fraction occupied by the char particles.

As the char undergoes reactions, slag associated with the matrix becomes free and forms slag droplets that are attached to the surface of the char particle. The ash is assumed to be 
homogeneously distributed in the coal particle. Based on the mass fraction of the ash and carbon, the amount of ash that gets exposed on the surface per mass of carbon reacting can be calculated. The mass conservation equation for the attached slag droplets is given by Eq. 4.2.

$$
\begin{aligned}
& \rho_{s l} \frac{\partial\left((1-\varepsilon)\left(1-\varepsilon_{s d}\right) \varepsilon_{s a}\right)}{\partial t}=-\rho_{s l} \frac{\partial\left((1-\varepsilon)\left(1-\varepsilon_{s d}\right) \varepsilon_{s a} U_{s}\right)}{\partial x}+(1-\varepsilon)\left(1-\varepsilon_{s a}\right)\left(1-\varepsilon_{s d}\right) \Gamma_{s-g} \omega_{a s h}- \\
& \sum_{n=1}^{4} p_{s l, n} M_{c d, n}
\end{aligned}
$$

Here, $\rho_{s l}$ is the slag density, $\omega_{a s h}$ is the ratio of ash to carbon mass fraction, $p_{s l, n}$ is the number of slag droplets detached per unit volume per unit time from the char surface corresponding to the size bin $n$ and $M_{c d, n}$ is the mass of the slag droplet of critical diameter corresponding to the size bin $n$. The second term on the right side of Eq. 4.2 represents the rate of formation of the slag droplets due to the heterogeneous reactions. The final term represents the sum of the rates of detachment of slag droplets into their respective size bins.

Detached slag droplets belong to one of the four size bins. The mass conservation equation for the slag droplets in each of the bins is given by Eq. 4.3.

$$
\rho_{s l} \frac{\partial\left((1-\varepsilon) \varepsilon_{s d} \varepsilon_{d, n}\right)}{\partial t}=-\rho_{s l} \frac{\partial\left((1-\varepsilon) \varepsilon_{s d} \varepsilon_{d, n} U_{s}\right)}{\partial x}+p_{s l, n} M_{c d, n}-\frac{4 m_{s l, d e p, n}}{D_{i}}
$$

$m_{s l, d e p, n}$ is the deposition flux of the slag droplets in bin size $n$. Eq. 4.3 is written for three of the four bins. In addition, a summation equation shown in Eq. 4.4 is written.

$$
\sum_{n=1}^{4} \varepsilon_{d, n}=1
$$

The overall detached slag mass conservation equation is shown in Eq. 4.5.

$$
\rho_{s l} \frac{\partial\left((1-\varepsilon) \varepsilon_{s d}\right)}{\partial t}=-\rho_{s l} \frac{\partial\left((1-\varepsilon) \varepsilon_{s d} U_{s}\right)}{\partial x}+\sum_{n=1}^{4} p_{s l, n} M_{c d, n}-\frac{4 \sum_{n=1}^{4} m_{s l, d e p, n}}{D_{i}}
$$

The continuum model tracks the mass of slag droplets in the bulk based on $p_{s l, n}$ and $m_{s l, d e p, n}$. It should be noted that in the previous chapter, $m_{s l, d e p, n}$ was assumed to be zero but that assumption is relaxed in the enhanced model. 


\subsubsection{Particle Model}

For calculating the term $p_{s l, n}$ used in the continuum model, a particle model is required. This model tracks the growth of the slag droplets on the char particle and helps to identify the locations of detachment and the detachment rate from each of the bin sizes. The particle model for calculating the term $p_{s l}$ has been presented for a single bin size earlier. The present model is an extension of this work, applied to multiple size bins. A particle size distribution is used as an input to the model and is assumed to be constant throughout the length of the gasifier. In the present framework, it is also assumed that the ash content of all the char particles in a control volume is constant and the growth and detachment phenomena of slag droplets from a char particle are similar for all char particles in the same control volume.

The number of slag droplets that belong to bin size $n$ that could detach from the char surface is termed as $w_{\text {det }, n, i}$. After the slag droplets detach, the residual mass left behind gets added to the slag generated in the next control volume. This mass of remaining slag is denoted by $M_{s r, n, i}$. $M_{s r, n, i}$ and $w_{d e t, n, i}$ are calculated using Eq. 4.6-4.7.

$$
\begin{gathered}
w_{d e t, n, i}=\left\lfloor\frac{\omega_{a s h, i} \Gamma_{s-g, i} V_{c h, i} \tau_{p, i}+M_{s r, n, i-1}}{M_{c d, n}}\right\rfloor \\
w_{d e t, n, i} V_{c d, n} \rho_{s l, i}+M_{s r, n, i}=\omega_{a s h} \Gamma_{s-g, i} V_{c h, i} \tau_{p, i}+M_{s r, n, i-1}
\end{gathered}
$$

Here, $\omega_{a s h, i}$ is the ratio of ash to carbon mass fraction, $\Gamma_{s-g, i}$ is the sum of all heterogeneous reactions on the carbon of the char particle, $V_{c h, i}$ is the char particle volume, $V_{c d, n}$ is the detachment volume of the slag in size bin $n$ and $M_{c d, n}$ is its corresponding mass.

The total slag mass that could possibly separate from the char surface per unit volume of the reactor for each of the size bins is denoted by $M_{s l, p o s s, n, i}$ and is calculated using Eq. 4.8. Based on the PSD, the total mass of slag that does finally separate into each of the bin sizes in a CV is calculated using Eq. 4.9.

$$
\begin{aligned}
M_{s l, p o s s, n, i} & =w_{d e t, n, i} N_{c h, i} M_{c d, n} \\
M_{s l, s e p, n} & =M_{s l, p o s s, n, i} f r_{n}
\end{aligned}
$$


Here, $f r_{n}$ is the input to the model and is the fraction of mass present in bin size $n$ over the total slag mass in the bulk. The sum of $f r_{n}$ for all bin sizes is unity. $N_{c h, i}$ is the number of char particles per unit volume in the CV and is calculated using Eq. 4.10, which is obtained directly from the definition of the volume fractions.

$$
N_{c h, i} V_{c h, i}=\left(1-\varepsilon_{i}\right)\left(1-\varepsilon_{s a, i}\right)\left(1-\varepsilon_{s d, i}\right)
$$

Finally, the slag detachment rate into each of the bins is calculated using Eqs. 4.11-4.12.

$$
\begin{gathered}
p_{s l, n, i} M_{c d, n} \tau_{p, i}=M_{s l, s e p, n} \\
\tau_{p, i}=\frac{\Delta x}{U_{s, i}}
\end{gathered}
$$

where, $\tau_{p, i}$ is the residence time of the particles in a CV. Eqs. 4.10-4.11 are the key equations for connecting the continuum and particle models. Eqs. 4.6-4.9 and Eq. 4.11 are written for each of the bin sizes. Additional equations are written for the shrinking char particles as shown in Eqs. 4.13-4.14.

$$
\begin{gathered}
M_{c h, i}=V_{c h, i} \rho_{c h} \\
M_{c h, i}=M_{c h, i-1}-\Gamma_{s-g, i} V_{c h, i} \tau_{p, i}\left(1+\omega_{a s h}\right)
\end{gathered}
$$

Figure 4.3 shows the important equations and exchange of information between the continuum model and the particle model. The continuum and particle model equations are solved simultaneously. 


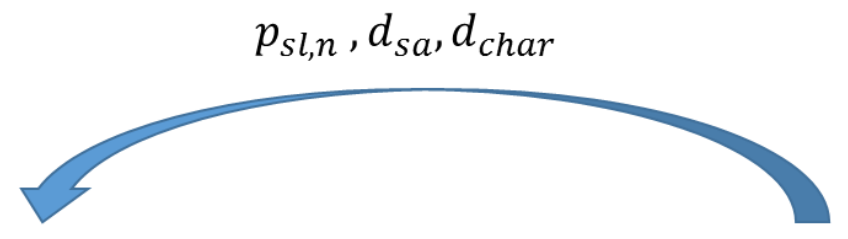

\section{Continuum model}

Overall mass conservation eqn.

Overall attached slag mass conservation eqn.

Overall detached slag mass conservation eqn.

Attached slag bin mass conservation eqn.

Detached slag bin mass conservation eqn.

\section{Particle model}

$$
N_{c h, i} V_{c h, i}=\left(1-\varepsilon_{i}\right)\left(1-\varepsilon_{s a, i}\right)\left(1-\varepsilon_{s d, i}\right)
$$$$
M_{c h, i}=V_{c h, i} \rho_{c h}
$$$$
M_{c h, i}=M_{c h, i-1}-\Gamma_{s-g, i} V_{c h, i} \tau_{i}\left(1+\omega_{a s h}\right)
$$

$$
\begin{gathered}
w_{d e t, n, i}=\left\lfloor\frac{\omega_{a s h} \Gamma_{s-g, i} V_{c h, i} \tau_{i}+M_{s r, n, i-1}}{M_{c d, n}}\right\rfloor \\
w_{d e t, n, i} V_{c d} \rho_{s l, i}+M_{s r, n, i}=\omega_{a s h} \Gamma_{s-g, i} V_{c h, i} \tau_{i}+M_{s r, n, i-1}
\end{gathered}
$$

$$
M_{s l, p o s s, n, i}=w_{s l, n, i} N_{c h, i} M_{c d, n}
$$$$
M_{s l, s e p, n}=M_{s l, p o s s, n, i} f r_{n}
$$

$$
p_{s l, n, i} M_{c d, n} \tau_{p, i}=M_{s l, s e p, n}
$$

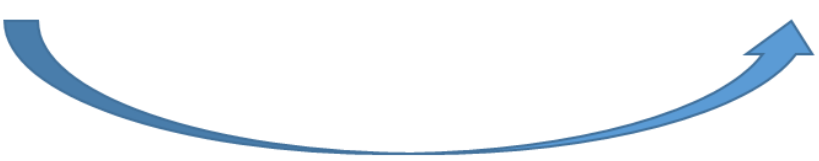

$$
\varepsilon, \varepsilon_{s a}, \varepsilon_{s d}, \Gamma_{s-g}, \omega_{a s h}, \tau_{i}
$$

Figure 4.3: Transfer of information between the particle model and continuum model 


\subsubsection{Slag Transport and Deposition}

While most of the slag droplets that are detached into the bulk remain in the gas phase, some of the droplets and char particles in the vicinity of the wall can impact the molten slag layer and get captured as shown in Figure 4.4. The deposition flux of the impacting particles and droplets is a key input required for the slag layer sub-model. The deposition flux depends on the number density of the particles and droplets as well as the deposition velocity.

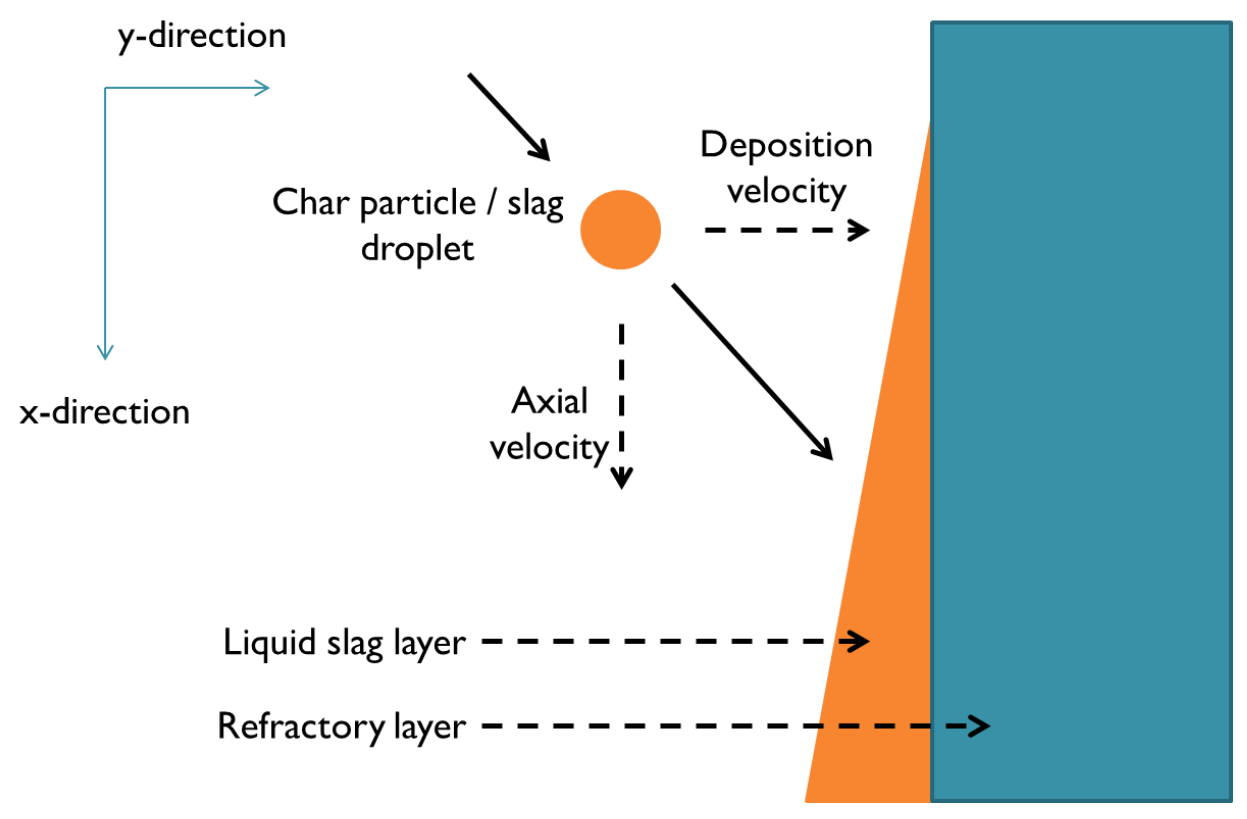

Figure 4.4: Schematic showing the slag droplet deposition on the gasifier wall

Since the present model is a 1-D model of the slagging gasifier, an analytical expression is required in order to calculate the velocity of the char particles and slag droplets. Experimental and computational works are available in the open literature where the transport and deposition of solid particles or liquid droplets suspended in a flowing fluid are studied. The results are usually presented in the form of a dimensionless velocity as a function of dimensionless relaxation time graph as discussed in section 2.1.4. The relaxation time is a function of flow conditions as well as particle density and size and is calculated using Eq. 4.15.

$$
\tau_{+}=\frac{\rho_{p} d_{p}{ }^{2} u_{*}^{2}}{18 \rho_{g} \vartheta^{2}}
$$


In Eq. 4.15, $\rho_{p}$ is the particle density, $d_{p}$ is the particle diameter, $u_{*}$ is the fluid friction velocity, $\rho_{g}$ is the gas phase velocity and $\vartheta$ is the kinematic viscosity. The deposition velocity is calculated using Eq. 4.16.

$$
V_{d e p}=V_{d e p+} u_{*}
$$

For particles depositing due to turbulent diffusion and eddy diffusion impaction, $V_{d e p+}$ is given by Eq. 4.17 .

$$
V_{d e p+}=\frac{3 \sqrt{3}}{29 \pi}\left(\frac{D_{p}}{\vartheta}\right)^{2 / 3}+4.5 \times 10^{-4} \tau_{+}^{2}
$$

where, $D_{p}$ is the particle diffusivity and is given by;

$$
D_{p}=\frac{k T}{3 \pi \mu d_{p}}
$$

In Eq. 4.18, $k$ is the Boltzmann's constant, $T$ is the absolute temperature and $\mu$ is the gas phase viscosity. The dimensionless deposition velocity for particles that deposit due to particle inertia can be calculated using Eq. 4.19. The dimensionless deposition velocity is assumed to be constant and independent of particle size (Wood, 1981).

$$
V_{d e p+}=0.175
$$

The fluid friction velocity and friction factor can be calculated using Eqs. 4.20-4.21 for the current range of fluid Reynolds number and are taken from Haaland (1983).

$$
\begin{gathered}
u^{*}=\left(\frac{f}{2}\right)^{.5} \bar{u} \\
\frac{1}{\sqrt{f}}=-1.8 \log _{10}\left[\left(\frac{\varepsilon_{*} / D}{3.7}\right)^{1.11}+\frac{6.9}{R e}\right]
\end{gathered}
$$

In the present work, it is assumed that the molten slag layer has a smooth surface and the term associated with surface roughness in Eq. 4.21 i.e. $\left(\varepsilon_{*} / D\right)$ is set to zero. 
Using Eqs. 4.17-4.21, the deposition velocity of the slag droplets in different bin sizes and char particles can be calculated. For the current flow conditions, it is found that slag droplets of the size 1-10 microns deposit due to turbulent diffusion and eddy diffusion impaction with values for $\tau_{+}$less than 15 . The slag droplets in the larger bin sizes have $\tau_{+}$values ranging from 50 to 400 , depending upon location in the gasifier and size droplet sizes. Droplets in these size bins fall into the particle inertia dominant regime. The char particles enter the gasifier at a size of 100 microns which correspond to $\tau_{+}$as high as 1000 . As the char particles react, they shrink in size and can exit the gasifier with $\tau_{+}$values lower than 50. As a result the char particles also fall in the particle inertia dominant regime and therefore have the same deposition velocity as the larger slag droplets as shown in Eq. 4.19.

The deposition flux terms used in Eqs. 4.1, 4.3 and 4.5 are calculated using Eqs. 4.22-4.24,

$$
\begin{array}{r}
m_{s l, d e p, n}=\frac{(1-\varepsilon) \varepsilon_{s d} \varepsilon_{d, n} \rho_{s l} V_{d e p, n}}{2} \\
m_{c h, d e p}=\frac{(1-\varepsilon)\left(1-\varepsilon_{s a}\right)\left(1-\varepsilon_{s d}\right) \rho_{c h a r} V_{d e p . c h a r}}{2} \\
m_{d e p}=m_{c h, d e p}+\sum_{n=1}^{4} m_{s l, d e p, n}
\end{array}
$$

Here, $V_{d e p, n}$ is the slag deposition velocity for different bin sizes and $V_{\text {dep,char }}$ is the char particle deposition velocity. The slag deposition velocity is calculated using the Sauter mean diameter of the size range of the bins. 


\subsubsection{Slag Flow Model}

It is important to ensure that the wall temperature in a refractory-lined entrained flow gasifier be high enough to avoid the formation of a solid slag layer. Slag solidification can lead to a rapid reduction in the available volume for reactions in the gasifier and can eventually clog the equipment. The maximum viscosity for slagging gasifiers is considered to be 250 Poise to avoid build-up of the slag layer (Wang et al., 2013). To investigate the dynamics of the slag layer on the wall, a liquid slag layer sub-model is incorporated into the gasifier model described previously. A linear temperature profile across the slag thickness, i.e., in the radial direction is assumed. An analytical expression for the momentum balance is used and the mass and energy balance equations are solved using continuum equations. Several heat transfer mechanisms have been considered in the energy balance equation of the slag layer including convective heat transfer between the gas and the slag layer, conductive heat transfer between the refractory wall and the slag layer and the radiative heat interaction between various sections of the inside wall of the gasifier, and the solid particles in the gasifier bulk, with the slag layer. Such an involved heat balance equation for the slag layer has not been considered previously in the literature to the best of the author's knowledge. A schematic of the slag model is shown in Figure 4.5.

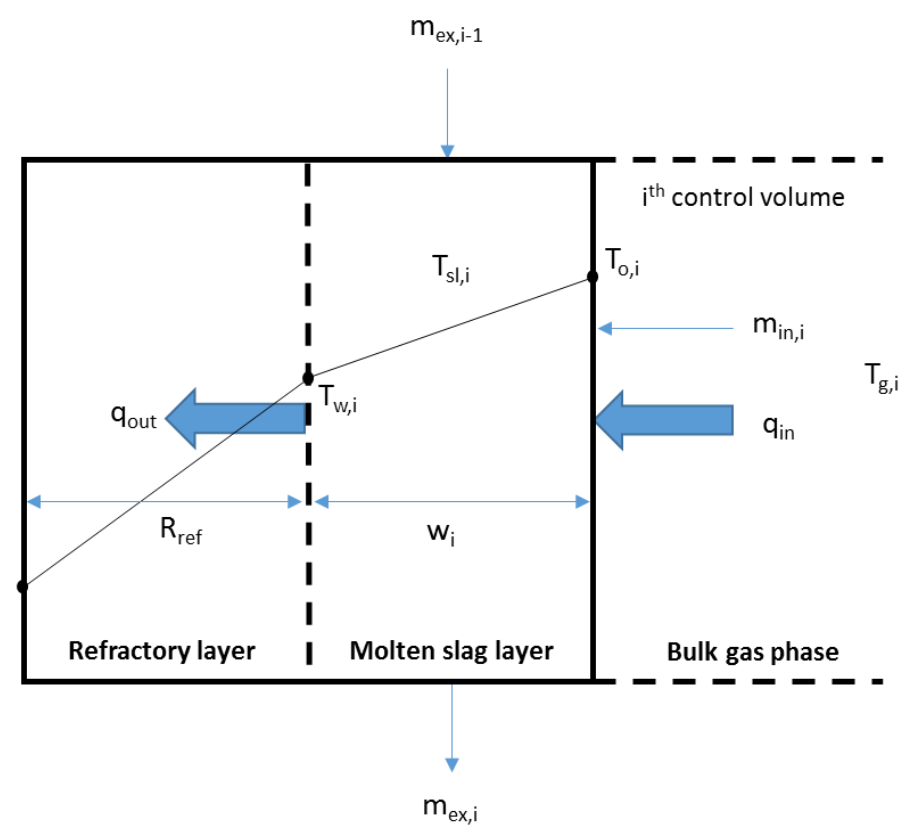

Figure 4.5: Schematic of the mass, momentum and energy interactions in the slag layer 
The mass balance equation is shown in Eq. 4.25 (Seggiani, 1998).

$$
\rho_{s l} \pi D_{i} \Delta x \frac{d w_{i}}{d t}=m_{i n, i}+m_{e x, i-1}-m_{e x, i}
$$

$m_{i n, i}$ is the mass deposition rate onto the control volume, $m_{e x, i}$ is the mass flow rate of slag flowing out of the control volume and $m_{e x, i-1}$ is the mass flow rate of slag flowing into the control volume and $w_{i}$ is the slag layer thickness. $m_{e x, i}$ is calculated using Eq. 4.26 (Bird et al., 2002).

$$
m_{e x, i}=\frac{1}{3} \frac{\pi D_{i} \rho_{s l}{ }^{2} g w_{i}^{3}}{\eta_{s l, i}}
$$

$\eta_{s l, i}$ is the viscosity of the slag in the slag flow layer and $g$ is acceleration due to gravity.

The expression for viscosity is calculated by the BCURA $S^{2}$ correlation (Streeter, 1985) using Eqs. 4.27-4.28.

$$
\begin{aligned}
& \log _{10} \eta_{s l}=4.468 s^{2}+\frac{12650}{T_{s l}}-7.44 \\
& s=\frac{\mathrm{SiO}_{2}}{\mathrm{SiO}_{2}+\mathrm{Fe}_{2} \mathrm{O}_{3}+\mathrm{CaO}+\mathrm{MgO}}
\end{aligned}
$$

where, $s$ is known as the silica ratio.

A slag layer heat balance equation is derived and shown in Eq. 4.29.

$$
\begin{aligned}
& \rho_{s l} w C_{p, s l, i} \pi D_{i} \Delta x \frac{d T_{s l, i}}{d t}=q_{i n, i}-q_{o u t, i}+m_{i n, i} C_{p, s l, i}\left(T_{s, i}-T_{s l, i}\right)+m_{e x, i-1} C_{p, s l, i}\left(T_{s l, i-1}-\right. \\
& \left.T_{s l, i}\right)
\end{aligned}
$$

where $C_{p, s l, i}$ is the specific heat, $q_{i n, i}$ is the sum of the energies flowing into the slag layer from the bulk side of the gasifier, $q_{o u t, i}$ is the heat conducted to the refractory, $T_{s l, i}$ and $T_{s l, i-1}$ are the temperatures of the slag layer in the current and previous control volume, respectively, and $T_{s, i}$ is the temperature of the incoming slag droplet, which is assumed to be equal to the solid phase temperature. $q_{i n, i}$ is calculated using Eqs. 4.30-4.35. The heat transfer mechanisms considered in this work are similar to Kasule et al. (2012). 


$$
\begin{gathered}
q_{i n, i}=\pi D_{i} \Delta x \cdot\left[q_{c o n v, s l-g}+q_{r a d, s l-p}+q_{r a d, s l-b}+q_{\text {rad }, s l-t o p}+q_{\text {rad,sl }}-s l_{\text {all } \neq l}\right] \\
q_{c o n v, s l-g}=h_{s l-g}\left(T_{o}-T_{g}\right) \\
q_{r a d, s l-p}=F_{s l-p} \sigma_{s l-p}\left(T_{o}^{4}-T_{s}^{4}\right) \\
q_{r a d, s l-b}=F_{s l-b} \sigma_{s l-b}\left(T_{o}^{4}-T_{o}(e n d)^{4}\right) \\
q_{r a d, s l-t o p}=F_{s l-t o p} \sigma_{s l-t o p}\left(T_{o}^{4}-T_{w}(0)^{4}\right) \\
q_{r a d, s l_{i}-s l_{a l l, a \neq l}}=\sum_{a} e_{s l} F_{s l_{i}-s l_{a}}\left(T_{o_{i}}^{4}-T_{o_{a}}^{4}\right)
\end{gathered}
$$

Here, $q_{c o n v, s l-g}$ is the transfer due to convection from the gas phase in the bulk, $q_{r a d, s l-p}$ is the radiation heat transfer between the particles to the slag layer, $q_{\text {rad,sl-b }}$ and $q_{\text {rad,sl-top }}$ are the radiation heat transfer between the slag layer and the top and bottom wall of the slagging gasifier respectively and $q_{r a d, s l_{i}-s l_{a l l, a \neq l}}$ is the radiation heat transfer between different control volumes in the slag layer. $F$ represents the view factor for the corresponding radiation heat flux terms. The equations for the friction factors are shown in Eqs. 4.36-4.39 and were obtained from Siegel and Howell.

$$
\begin{gathered}
F_{s l-p}=\left[\left(\left(z_{s l-p} / D_{i}\right)^{2}+0.5\right) / \sqrt{\left(z_{s l-p} / D_{i}\right)^{2}+1}\right]-\left(z_{s l-p} / D_{i}\right) \\
F_{s l-b}=\left[\left(\left(z_{s l-b} / D_{i}\right)^{2}+0.5\right) / \sqrt{\left(z_{s l-b} / D_{i}\right)^{2}+1}\right]-\left(z_{s l-b} / D_{i}\right) \\
F_{s l-t o p}=\left[\left(\left(z_{s l-t o p} / D_{i}\right)^{2}+0.5\right) / \sqrt{\left(z_{s l-t o p} / D_{i}\right)^{2}+1}\right]-\left(z_{s l-t o p} / D_{i}\right) \\
\left.\left.F_{s l_{i}-s l_{a l l, a \neq l}=1-\left[1-\left[\left(2\left(z_{s l-b} / D_{i}\right)^{3}+3\left(z_{s l-b} / D_{i}\right)\right) /\left(2\left(z_{s l-b} / D_{i}\right)^{2}+1\right)\right.\right.}\right]^{1.5}\right] d z
\end{gathered}
$$

$z$ is the distance between the surfaces. $T_{o}$ in Eqs. 4.31-4.35 is the temperature at the hot face of the slag layer and is given by Eq. 4.40 (Seggiani, 1998). qout, $i$ is obtained using Eq. 4.41.

$$
\begin{gathered}
T_{o}=2 T_{s l}-T_{w} \\
q_{\text {out }, i}=2 \pi \Delta x .\left(T_{o}-T_{w}\right) \lambda_{s l}
\end{gathered}
$$




\subsection{Computational Approach}

The slagging gasifier model, the slag transport and deposition model, and the model for the slag layer are integrated and solved using Aspen Custom Modeler ${ }^{\circledR}$ (ACM). In this formulation, $w_{d e t, n, i}$ is an integer variable, which makes it difficult to solve the problem in software like ACM. In order to obtain a solution, an offline calculation is first done to obtain initial values of $w_{\text {det }, n, i}$ for the solver. This approach worked for obtaining steady state solutions, however, for a dynamic simulation, this approach is not feasible. The error in results is assessed by assuming that the detachment process is continuous rather than discrete for a number of cases. The error was found to be acceptable for slag droplets with detachment diameters of 1-10 micron. Therefore, the resulting error in calculation of the deposition flux is expected to be small since only a fraction of the detached droplets are deposited. The assumption of continuous detachment of slag droplets can also be extended to larger slag droplets if the deposition velocities of these droplets and char particles are the same. This is because the total amount of slag deposited is calculated by summing up the slag deposition due to impaction of both char particles and slag droplets. As mentioned previously, as both large slag droplets and char particles belong to the particle inertia dominated regime, their deposition velocities are expected to be similar.

Due to the assumption of continuous detachment, Eq. 4.2 is replaced by Eq. 4.42.

$$
\varepsilon_{s a}=0
$$

The slag mass does not remain on the surface and continually detaches into the slag size bins in the bulk according to the particle model. The slag volume fraction in each bin is calculated using Eq. 4.38. The total detached slag fraction can be calculated by summing up Eq. 4.43 for all size bins and is given as Eq. 4.44. Therefore, Eq. 4.3 and Eq. 4.5 are replaced by Eq. 4.43 and Eq. 4.44 .

$\rho_{s l} \frac{\partial\left((1-\varepsilon) \varepsilon_{s d} \varepsilon_{d n}\right)}{\partial t}=-\rho_{s l} \frac{\partial\left((1-\varepsilon) \varepsilon_{s d} \varepsilon_{d n} U_{s}\right)}{\partial x}+(1-\varepsilon)\left(1-\varepsilon_{s a}\right)\left(1-\varepsilon_{s d}\right) \Gamma_{s-g} \omega_{a s h} f r_{n}-\frac{4 m_{s l, d e p, n}}{D_{i}}$

$\rho_{s l} \frac{\partial\left((1-\varepsilon) \varepsilon_{s d}\right)}{\partial t}=-\rho_{s l} \frac{\partial\left((1-\varepsilon) \varepsilon_{s d} U_{s}\right)}{\partial x}+(1-\varepsilon)\left(1-\varepsilon_{s a}\right)\left(1-\varepsilon_{s d}\right) \Gamma_{s-g} \omega_{a s h}-\frac{4 \sum_{n=1}^{4} m_{s l, d e p, n}}{D_{i}}$ 
It should be noted that by using a larger number of bins or a detailed particle size distribution and more accurate calculation of deposition velocities, especially in the particle inertia regime, a more accurate value for the slag thickness can be obtained. However considering both accuracy and computational tractability for a dynamic simulation, the authors believe that the current approach is reasonable. 


\subsection{Model Input and Simulation}

The dimensions of the gasifier used in the present model are taken from the literature for the TECO gasifier. The dimensions along with the operating conditions are shown in Table 4.1. The base coal type used in the steady state and dynamic simulation of the slagging gasifier model is Illinois \#6. A dynamic run where the coal type is changed from Illinois \#6 to Pittsburgh \#8 is also simulated. The proximate and ultimate analyses for both the coal types are shown in Table 4.2 .

Table 4.1: Model parameters and input conditions

\begin{tabular}{cc}
\hline Parameters/Conditions & Value \\
\hline Gasifier parameters & 6.62 \\
\hline Length $(\mathrm{m})$ & 1.79 \\
Internal diameter $(\mathrm{m})$ & \\
Operating conditions & 220,438 \\
\hline Coal slurry flow rate $(\mathrm{kg} / \mathrm{hr})$ & 100 \\
Particle diameter $(\mu \mathrm{m})$ & 0.4115 \\
Water to coal ratio & 0.8347 \\
O 2 to coal ratio & 29.85 \\
Inlet Temperature $\left({ }^{\circ} \mathrm{C}\right)$ & 28.33 \\
Inlet Pressure $(\mathrm{bar})$ & 1.5 \\
Recirculation ratio & \\
\hline
\end{tabular}


Table 4.2: Proximate and Ultimate analysis of Illinois \#6 and Pittsburgh \#8 coal [As-Received (wt \%)]

\begin{tabular}{lcc}
\hline \multicolumn{1}{c}{ Analyses } & Illinois \#6 & Pittsburgh \#8 \\
\hline Proximate analysis & 44.19 & 52.38 \\
Fixed Carbon & 9.99 & 9.17 \\
Ash & 34.70 & 35.82 \\
Volatile matter & 11.12 & 2.63 \\
Moisture & & \\
& & \\
Ultimate analysis (DAF) & 63.75 & 73.15 \\
C & 4.50 & 4.97 \\
H & 6.88 & 6.22 \\
O & 1.25 & 1.46 \\
N & 2.51 & 2.36 \\
S & & 0.6105 \\
Silica ratio & 0.5266 & \\
\hline
\end{tabular}


The control structure for the dynamic runs is shown in Figure 4.6.

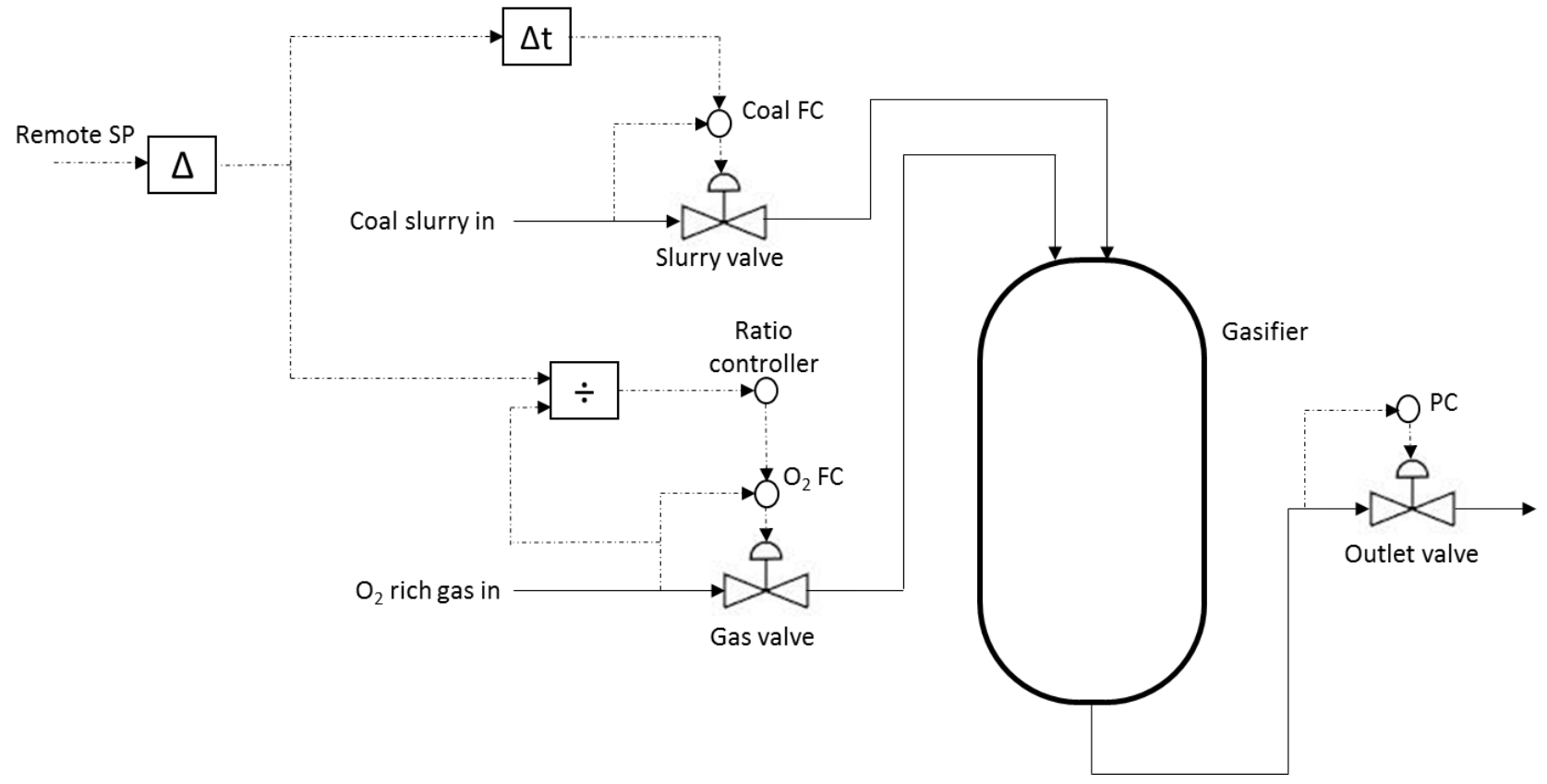

Figure 4.6: Control structure implemented to simulate dynamic runs for the slagging gasifier

To avoid an oxygen-rich environment in the gasifier when the coal flow needs to be increased, first the coal flow is increased and then oxygen flow is increased. While decreasing the coal flow, first oxygen flow is increased. An $\mathrm{O}_{2} /$ coal ratio controller is used to generate the set point for the oxygen flow controller. The gasifier pressure is controlled by a valve in the exit line. It should be noted that for an IGCC plant, the gasifier pressure is controlled depending on the control strategy that is used. For a gas-turbine-lead-gasifier-follow strategy, the gasifier pressure is controlled by manipulating the slurry flowrate to the gasifier. For the gasifier-lead-gas-turbinefollow strategy the gasifier pressure is controlled by manipulating the syngas flow to the gas turbine. The current control system setup mimics the later strategy, but the pressure controller is placed right at the gasifier outlet as the balance of the plant is not considered in this study. 


\subsection{Results}

The partial differential algebraic equation (PDAE) based model is solved in ACM using the method of lines. The partial differential equations are discretized into 250 grids along its length using backward finite difference (Kasule et al., 2012; Kasule et al., 2014). Gear's method is used for integration. A total number of 48,515 equations are solved simultaneously. The input parameters used in the slag model are shown in Table 4.3.

Table 4.3: Parameters for slag flow layer

\begin{tabular}{|c|c|c|c|}
\hline Parameter & Value/Expression & Units & Source \\
\hline Silica ratio & 0.527 & - & Rees (1964) \\
\hline Viscosity & $10^{\wedge}\left(4.468 \mathrm{~s}^{2}+\left(12650 / \mathrm{T}_{\mathrm{sl}}\right)-7.44\right.$ & Poise & Streeter et al. (1985) \\
\hline Density & 1.9 & $\mathrm{~g} / \mathrm{cm}^{3}$ & Kasule (2012) \\
\hline Specific heat & 0.4039 & $\mathrm{cal} / \mathrm{g} . \mathrm{K}$ & Seggiani (1998) \\
\hline Thermal conductivity & 0.00451 & $\mathrm{cal} / \mathrm{s} . \mathrm{cm} . \mathrm{K}$ & Seggiani (1998) \\
\hline Emissivity & 0.83 & - & Seggiani (1998) \\
\hline
\end{tabular}

\subsubsection{Model Validation}

The slagging gasifier model without considering slag transport, deposition, and slag flow layer has been validated in our previous work (Pednekar et al., 2016). Data for the slag layer of a commercial scale slagging gasifier are scarce in the open literature as it is extremely difficult, if not impossible, to obtain using current measurement technology. Therefore, for model validation one option is to consider the computational models published in this area. While some CFD models (Benyon, 2002; Seggiani, 1998; Bockelie, 2002) have been developed for upward-firing, membrane-cooled slagging gasifiers, very little information exists on slag layer thickness or deposition rate for refractory-walled downward-firing gasifiers. Table 4.4 shows validation of the present slagging gasifier model under steady state conditions. Two variables, deposition $\%$ and slag layer thickness, are compared with the results available in two references (Bockelie, 2002; Monaghan, 2012) that have developed CFD models. However, as noted before, these CFD models consider char deposition as the only mechanism for slag deposition.

Bockelie et al. (2002) simulated a CFD model of a downward-firing commercial scale gasifier fed with Illinois \#6 coal using similar operating conditions as the current work. The fraction of solid mass flow entering the gasifier that subsequently gets deposited is reported in their work. 
As Bockelie considered only char deposition, a complete coalescence case (i.e., no slag detachment) is considered for our model so that the results from our model can be compared with the work of Bockelie (2002). As seen in Table 4.4, the fraction of the total solid mass entering the gasifier that gets deposited is comparable for both the models. In the current model, $2 \%$ of the total solid mass entering the gasifier gets deposited onto the walls of the gasifier. It should be noted that the dimensions of the gasifier in our work are somewhat different than the work of Bockelie et al. (2002), where the $L / D$ ratio was considered to be 2 .

Table 4.4: Comparison of the results from this work with the existing literature (Bockelie, 2002;

Monaghan, 2012)

\begin{tabular}{cccccc}
\hline Source & $\begin{array}{c}\text { Inlet coal } \\
\text { flowrate }(\mathrm{kg} / \mathrm{h})\end{array}$ & $\begin{array}{c}\text { Gasifier } \\
\text { diameter }(\mathrm{m})\end{array}$ & $\begin{array}{c}\text { Gasifier } \\
\text { length }(\mathrm{m})\end{array}$ & $\begin{array}{c}\text { Deposition } \\
\%\end{array}$ & $\begin{array}{c}\text { Slag layer } \\
\text { thickness }(\mathrm{mm})\end{array}$ \\
\hline $\begin{array}{c}\text { Bockelie et al. } \\
(2002)\end{array}$ & 125,000 & - & - & 2.7 & - \\
$\begin{array}{c}\text { Monaghan \& } \\
\text { Ghoniem } \\
(2012)\end{array}$ & 113,586 & 2.74 & 8.31 & 10 & $6-7$ \\
Present model & 156,251 & 1.79 & 6.62 & 2.02 & 3.11 \\
\hline
\end{tabular}

Monaghan and Ghoniem (2012) developed a dynamic, reduced order model for a commercial scale gasifier. Using a silica ratio similar to their work, the current model shows that the slag layer thickness is expected to be much lower as seen in Table 4.4. One reason for this difference between our results and the work of Monaghan and Ghoniem (2012) is due to the difference in the estimated slag layer temperature. The average refractory wall temperature in the current model is about $120^{\circ} \mathrm{C}$ higher than the work of Monaghan and Ghoniem (2012) leading to a decrease in the slag layer thickness due to a lower viscosity. This could be due to difference in the operating conditions and the energy conservation model. The energy conservation model used in this work is similar to the comprehensive model developed by Kasule et al. (2012), which considers additional radiative heat transfer mechanisms in between the wall segments. When the refractory wall temperature in the present model was reduced to similar values in the work of Monaghan and Ghoniem (2012), it was found that the slag layer thickness increased from 3.1 to $4.5 \mathrm{~mm}$. Another reason for the difference in slag layer thickness is because Monaghan and Ghoniem (2012) assumed that $10 \%$ of total solid mass entering the gasifier is 
deposited on the wall while the current model makes no such assumption. No literature was found to support this number.

\subsubsection{Steady State Simulation Results}

\subsubsection{Effect of PSD}

Based on the slag droplet sizes that can be expected from detachment of included ash, the PSD is divided into four bin sizes. It is difficult to obtain a good estimate of the fraction of the slag droplets in each size bin along the gasifier. Since there is significant uncertainty in the estimated fraction of slag droplets in each bin, it was necessary to perform sensitivity studies by changing these fractions. Three different cases shown in Table 4.5 were evaluated. In Table 4.4, the variables $f r_{1}, f r_{2}, f r_{3}$ and $f r_{4}$ denote the mass fraction of the total slag in the 1-10 microns, 10-20 microns, 20-30 microns, and 30+ microns size bins, respectively. Obviously, for the complete coalescence case, denoted by case CC, all these fractions are zero.

Table 4.5: Simulated particle size distributions

\begin{tabular}{lcccc}
\hline \multicolumn{1}{c}{ PSD case } & $f r_{1}$ & $f r_{2}$ & $f r_{3}$ & $f r_{4}$ \\
& & & & \\
\hline SD35-10 & 0.35 & 0.30 & 0.25 & 0.10 \\
SD60-10 & 0.60 & 0.20 & 0.10 & 0.10 \\
SD5-50 & 0.05 & 0.15 & 0.30 & 0.50 \\
CC & 0 & 0 & 0 & 0 \\
\hline
\end{tabular}

Case SD35-10 is used as a base case for the model. The cases SD60-10 and SD5-50 are considered as limiting cases where the majority of the slag mass is considered to be in the small and large size bins, respectively.

Figure 4.7 shows the char and slag droplet deposition fluxes separately as well as the slag layer thickness profile along the gasifier for the base case PSD. It should be noted that for char particles impacting the gasifier wall, the char flux only represents the ash being added to the slag layer and the carbon continues to burn at the same rate as in the bulk of the gasifier. 


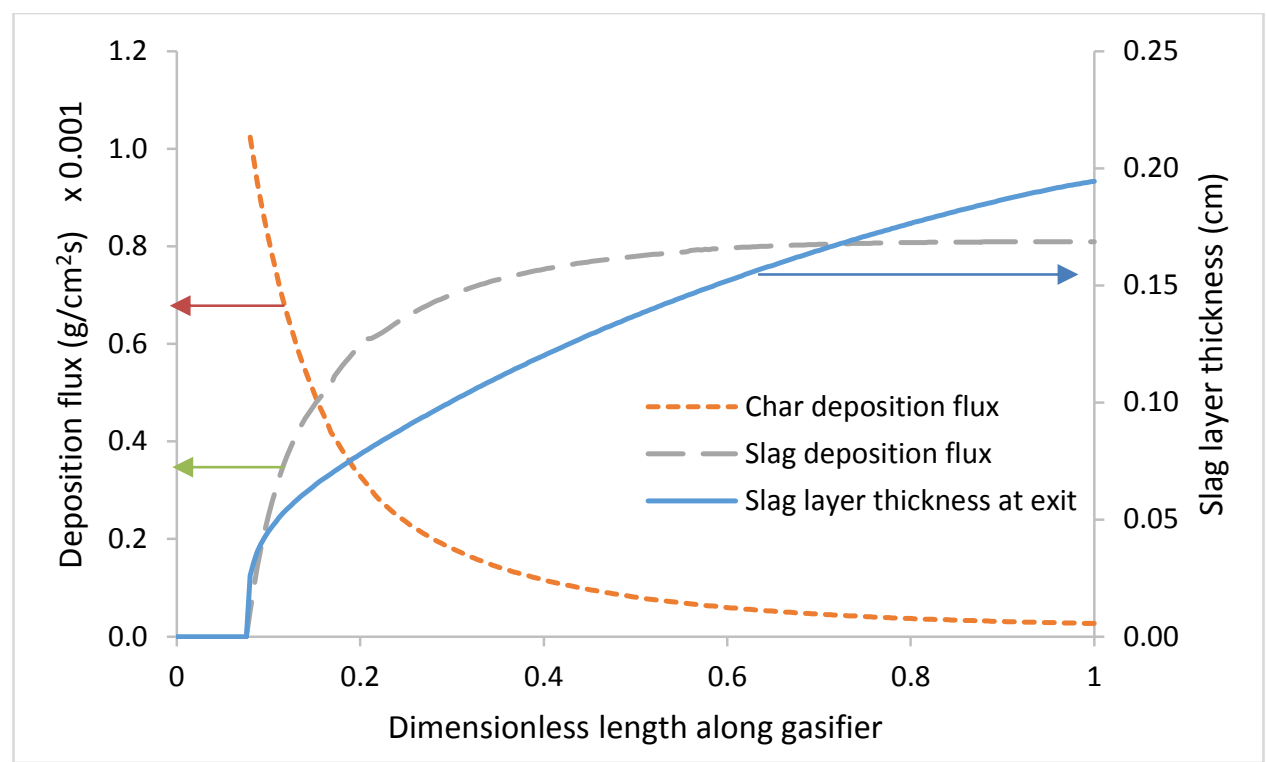

Figure 4.7: Deposition flux and slag layer thickness profile for base case PSD

Figure 4.7 shows that the slag layer thickness rapidly increases in the initial section of the gasifier and that slag addition due to the impact of char particles is the dominant mode of slag addition in this section. This is because the deposition velocity in the initial section is high and also because very little ash has separated as slag droplets from the char particles into the bulk of the gasifier. As a result, the amount of ash content in the impacting char particles is high. As the char particles react, increasing amounts of slag droplets are separated into the bulk and slag deposition becomes the dominant mode of slag addition to the layer. Towards the end of the gasifier, char impact adds little to the growth of the slag layer. In total, about $82 \%$ contribution to the slag layer comes from slag droplet impact and the rest from char impact. As stated previously, no slag is assumed to deposit onto the wall in the shrinking core section of the model.

Figure 4.8 shows the slag droplet deposition flux and the slag layer thickness for Case SD5-50 (PSD with higher mass fraction of larger particles) and for Case SD60-10 (PSD with higher mass fraction of smaller particles). The char deposition flux, not shown here, was found to be identical for the PSD cases. Even though the number density of the smaller slag droplets is higher, the deposition velocity and mass of the smaller slag droplets are lower in comparison to the larger slag droplets. Initially the slag layer thickness profile is similar since ash deposition due to char impaction is dominant. The profile begins to differ as the ash deposition begins to dominate. However the slag layer thickness does not differ appreciably. 


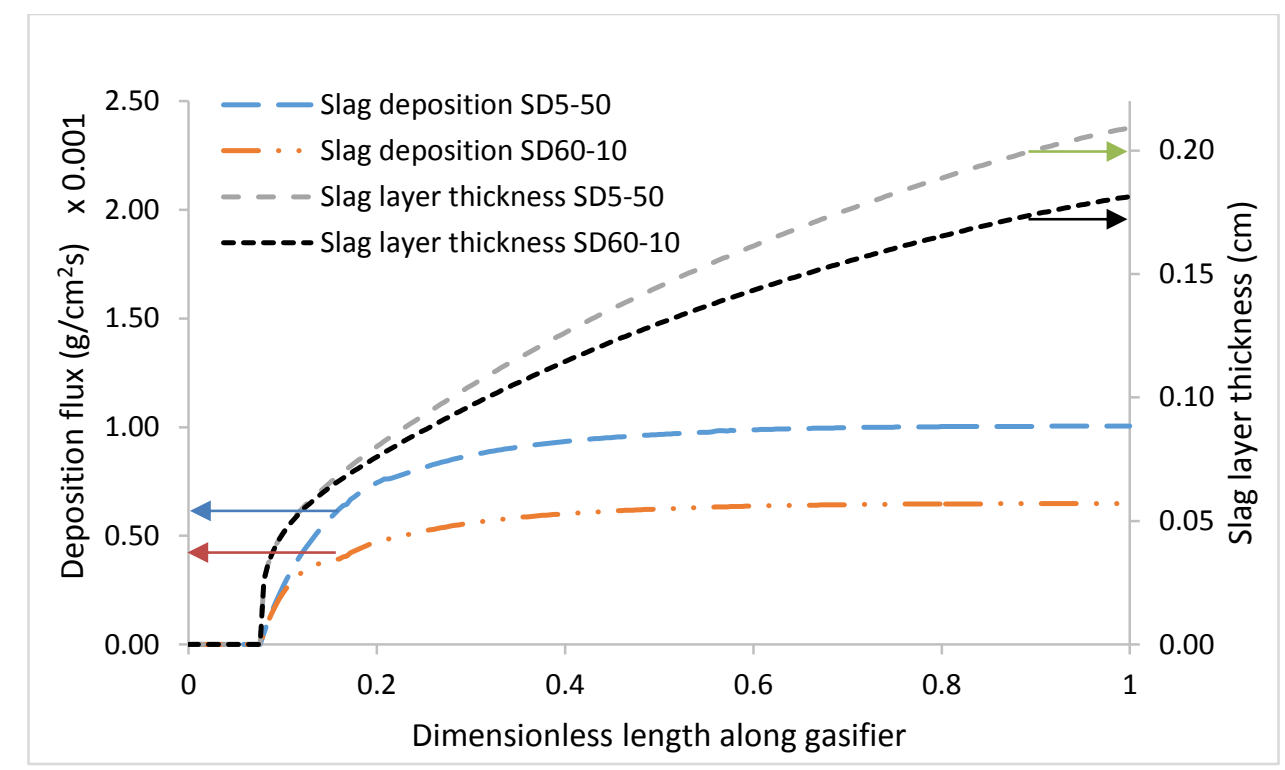

Figure 4.8: Slag droplet deposition flux and slag layer thickness for cases SD-5-50 and SD 60-10

Figure 4.9 shows the slag layer thickness profile for the Case CC in comparison to the base case. The difference in slag layer thickness is small.

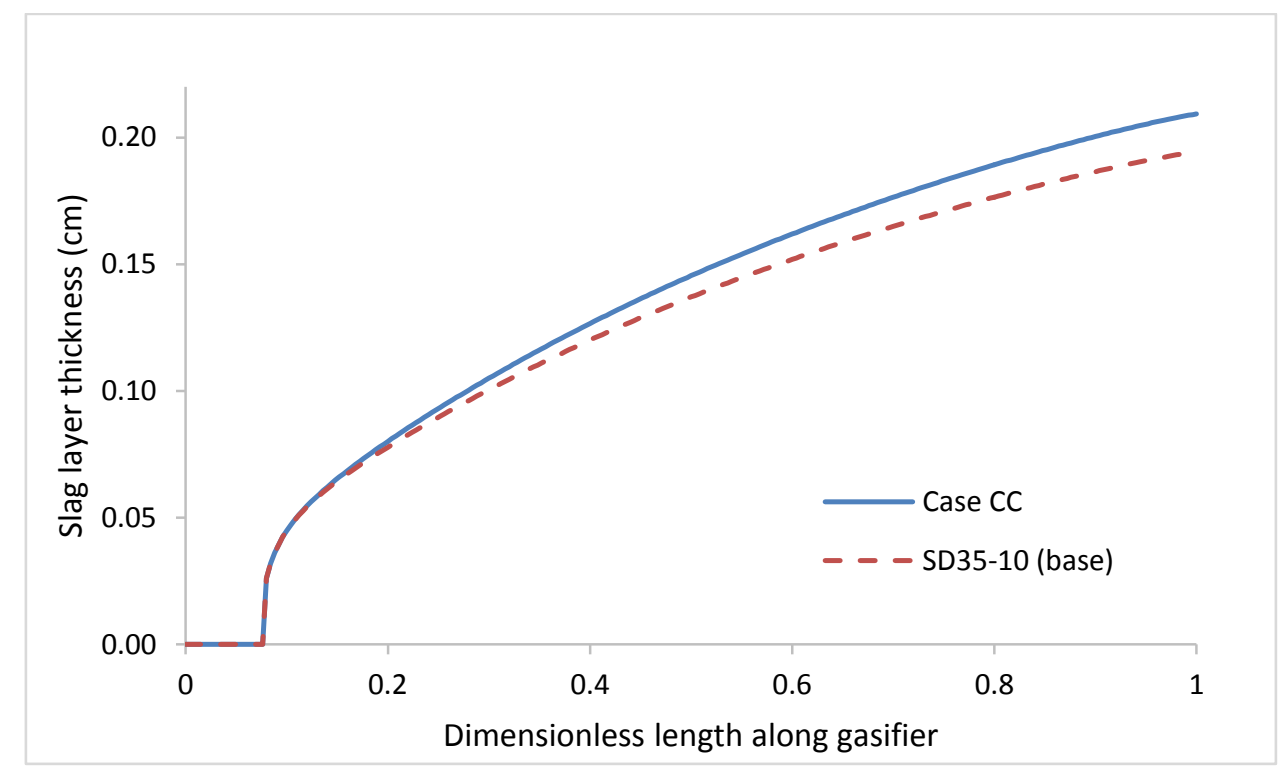

Figure 4.9: Comparison of the slag layer thickness for Case CC and base case

From Figures 4.7-4.9, it was found that the PSD did not have a significant effect on the slag layer thickness for the range of slag droplet sizes that were considered in this study. However, since it is has been shown that the ash can separate from char particles as slag droplets and these droplets 
could vary in sizes, the inclusion of a PSD for the detached slag droplets would be a more physically realistic representation of the system. To the best of the author's knowledge, the present formulation has not been done previously and this work could be useful to evaluate the deposition flux and slag layer thickness for the various cases at other operating conditions. For the subsequent runs, the base case PSD is used.

\subsubsection{Effect of Change in Input Conditions}

Disturbances in the $\mathrm{O}_{2}$ or coal flow rate can result in the slag layer temperature dropping below its critical viscosity, leading to thickening of the slag layer. The effect of change in $\mathrm{O}_{2} /$ coal ratio on the slag layer thickness can be seen in Figure 4.10.

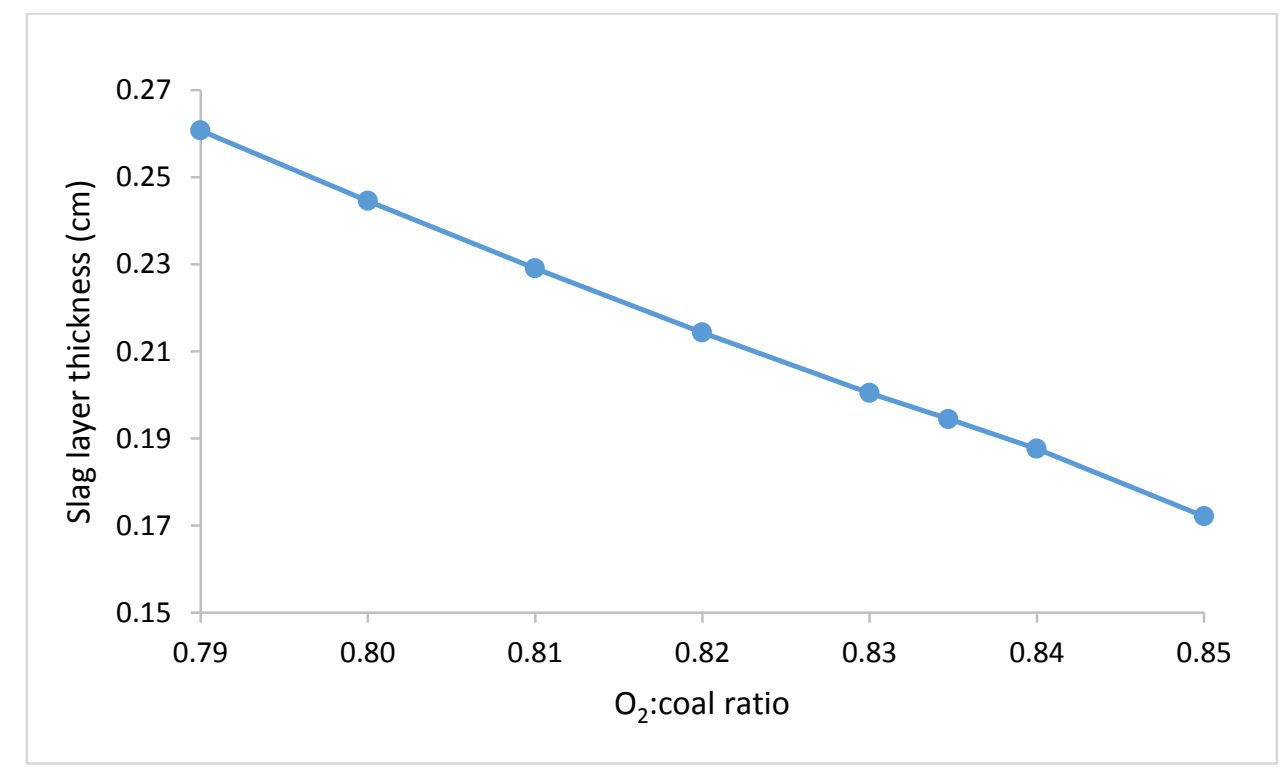

Figure 4.10: Effect of change in $\mathrm{O}_{2} / \mathrm{coal}$ ratio on slag layer thickness at gasifier exit

Changes in the $\mathrm{O}_{2} /$ coal ratio affect the gasifier bulk temperature and thus the slag layer temperature resulting in a change in its viscosity. With a change in the ratio from 0.79 to 0.85 , it is seen that the slag layer thickness decreases by approximately 35\%. (Pednekar et al., 2015)

The effect of change in the $\mathrm{O}_{2} /$ coal ratio on the maximum and minimum slag temperatures and carbon conversion is shown in Figure 4.11. The maximum temperature occurs near the gasifier inlet while the minimum temperature occurs at the exit. 


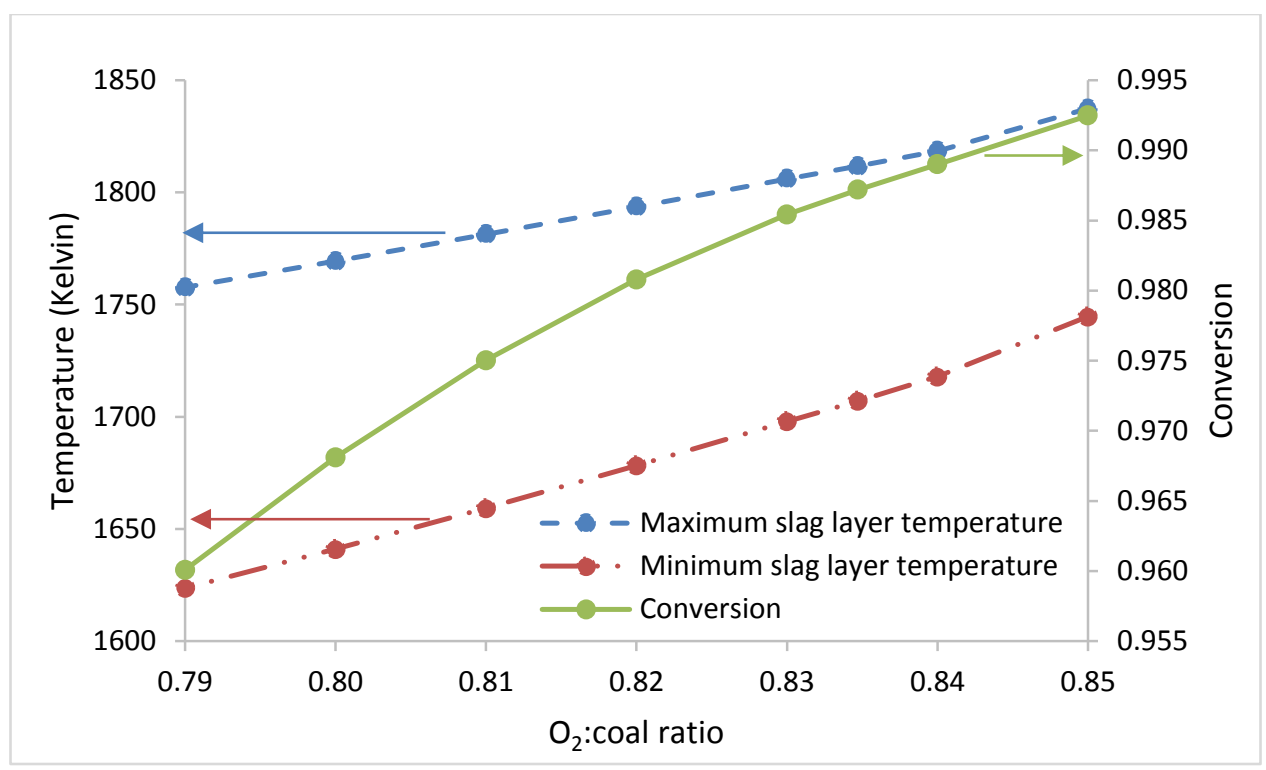

Figure 4.11: Variation of the maximum and minimum slag layer temperature due to change in $\mathrm{O}_{2} /$ Coal feed ratio

For Illinois \#6 the fluid temperature was found to be $1600 \mathrm{~K}$ in a reducing environment (Wu et al., 1999). If the $\mathrm{O}_{2} /$ coal ratio is decreased below 0.79 , the slag may cease to flow because of the lower temperature and higher slag viscosity. Even though it is observed in Figure 4.10 and Figure 4.11 that a higher $\mathrm{O}_{2}$ /coal ratio results in higher carbon conversion and lower slag layer thickness, the resulting high temperature has strong detrimental effect on the refractory life. Thus the $\mathrm{O}_{2} /$ coal ratio should be optimally controlled by evaluating these tradeoffs.

\subsubsection{Effect of Change in Ash Composition}

The composition and amount of ash can vary widely between coal types as well as for the same coal from different seams. Since the viscosity at a given temperature strongly depends on the ash composition, ash composition needs to be carefully considered during gasifier operation. In Table 4.6, the silica ratio of Illinois \#6 coal from different authors is presented. A silica ratio of 0.527 is used as a base case and is calculated for "Lab No. Christian c-10142" taken from a report of Illinois \#6 coal (Rees, 1964). 
Table 4.6: Silica ratios calculated for Illinois \#6 coal taken from literature

\begin{tabular}{cc}
\hline Source & Silica ratio \\
\hline Present model & 0.527 \\
McCollor et al. (1993) & 0.627 \\
Nowok (1994) & 0.690 \\
Cho et al. (2009) & 0.700 \\
\hline
\end{tabular}

The coal composition in this study is kept constant in order to assess the effect of changing only the silica ratio. The effect of silica ratio on slag layer thickness and exit viscosity can be seen in Figure 4.12 for the base case operating conditions shown in Table 4.1.

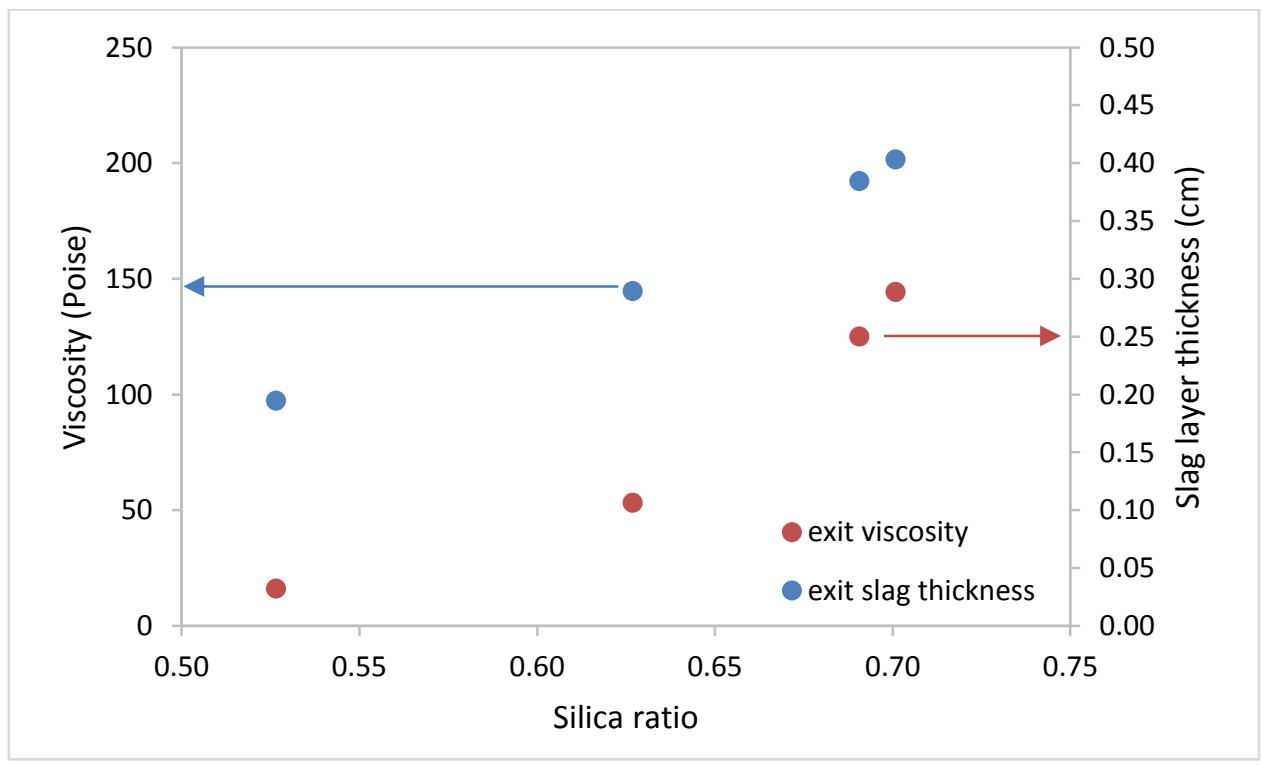

Figure 4.12: Effect of silica ratio on exit viscosity and slag layer thickness

It can be seen that there is a strong variation in slag layer thickness and viscosity due to a change in the silica ratio. Under the present operating conditions, the exit slag temperature is found to be about $1417^{\circ} \mathrm{C}$. Figure 4.12 shows that even though all operations in the given range are feasible, the slag layer thickness can more than double depending on the silica ratio. 


\subsubsection{Dynamic Simulation Results}

\subsubsection{Change in Coal Slurry Flowrate}

To study the effect of change in the gasifier throughput on slag layer thickness, the coal slurry flow rate was ramped up by $10 \%$ for a duration of 10 minutes. The oxygen rich air flow rate also is ramped by the ratio controller to maintain the desired $\mathrm{O}_{2} /$ coal ratio. The change in the flow rates is shown in Figure. 4.13. The slag layer thickness is found to increase by about 6\%. This increase happens due to two reasons. First, the overall mass flux of char particles and slag droplets to the wall increases. Second, there is also a small increase in the slag layer viscosity due to a decrease in the temperature of the slag layer at the exit of the gasifier. The transient temperature profiles of the temperature of the slag layer and the wall at the end of the gasifier are shown in Figure 4.14. While the slag layer temperature is responsive to the O2/coal ratio entering the gasifier, the wall temperature has a much slower dynamic response. The final temperature is lower, but by a small amount.

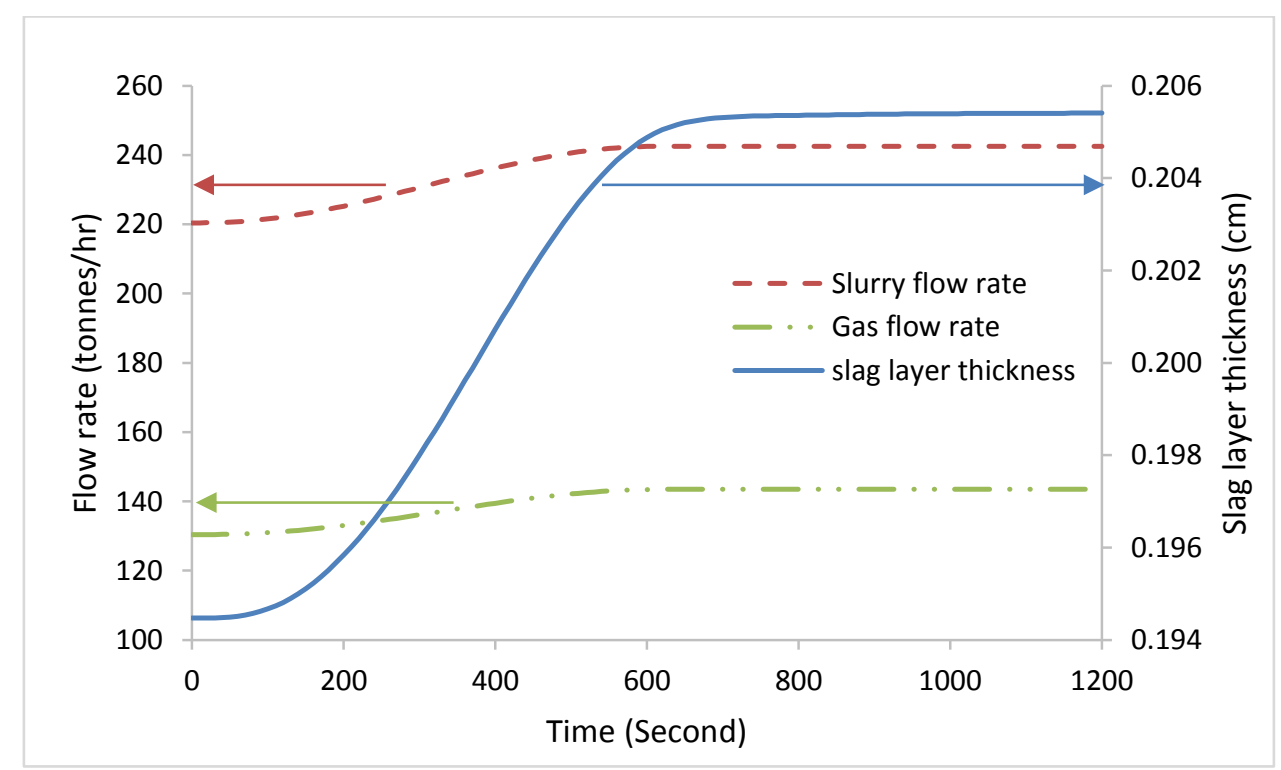

Figure 4.13: Effect of ramp increase in coal slurry flow rate on slag layer thickness at final CV 


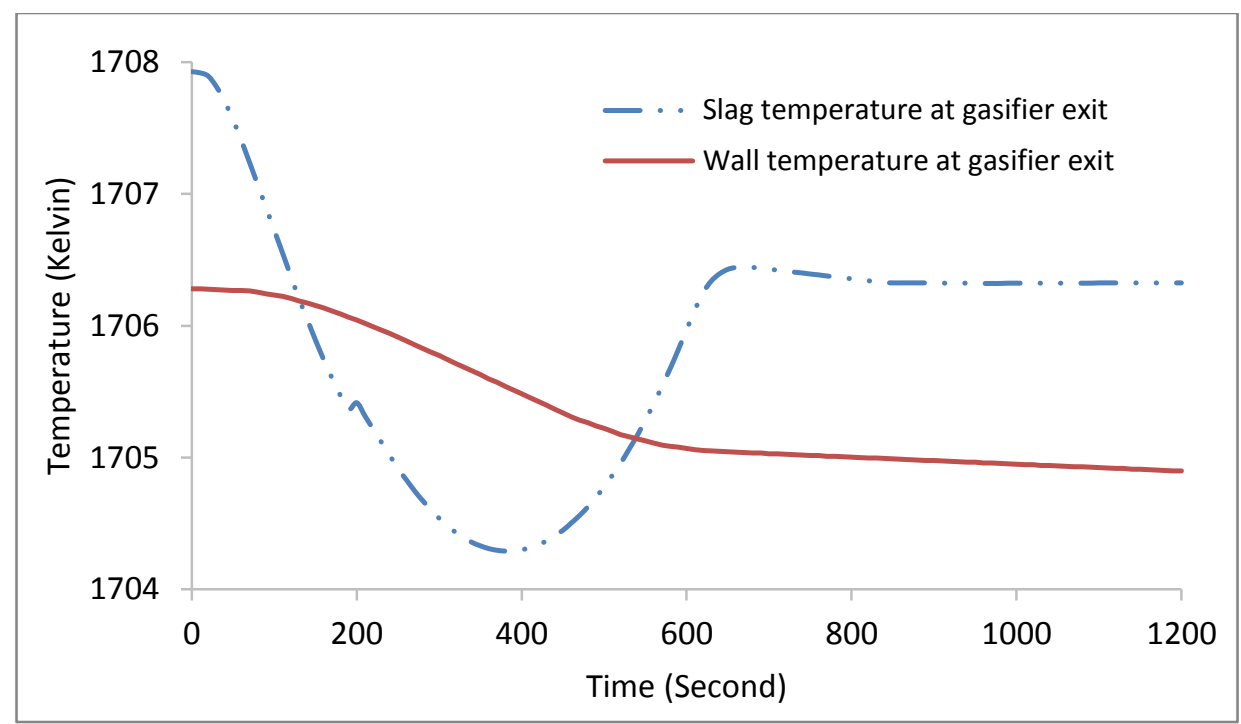

Figure 4.14: Effect of ramp increase in coal slurry flow rate on slag layer temperature at final CV

\subsubsection{Coal Feed Switch}

In this study, the coal feed to the gasifier is changed from Illinois \#6 to Pittsburgh \#8 over a period of 1 hour. The coal switch is initiated after 30 seconds of operation, and is achieved by ramping the normalized ultimate and proximate analysis parameters. These parameters for the two coals have been reported in Table 4.2. The $\mathrm{O}_{2} /$ coal ratio and the coal/water ratio is left unchanged to observe the effect of only the change in the coal type. It should be noted that usually during a coal switch, the $\mathrm{O}_{2} /$ coal ratio and coal/water ratio are normally adjusted and if these ratios are adjusted, the results would vary. The silica ratio is also ramped accordingly. For Pittsburgh \#8 coal, the silica ratio is calculated on the basis of ash composition available in a report from the U.S. DOE's National Energy Technology Laboratory (Vaysman et al., 2012).

Figure 4.15 shows the change in the mole fraction and carbon conversion due to switching the coal. The carbon conversion decreases from about $99 \%$ to $93 \%$. 


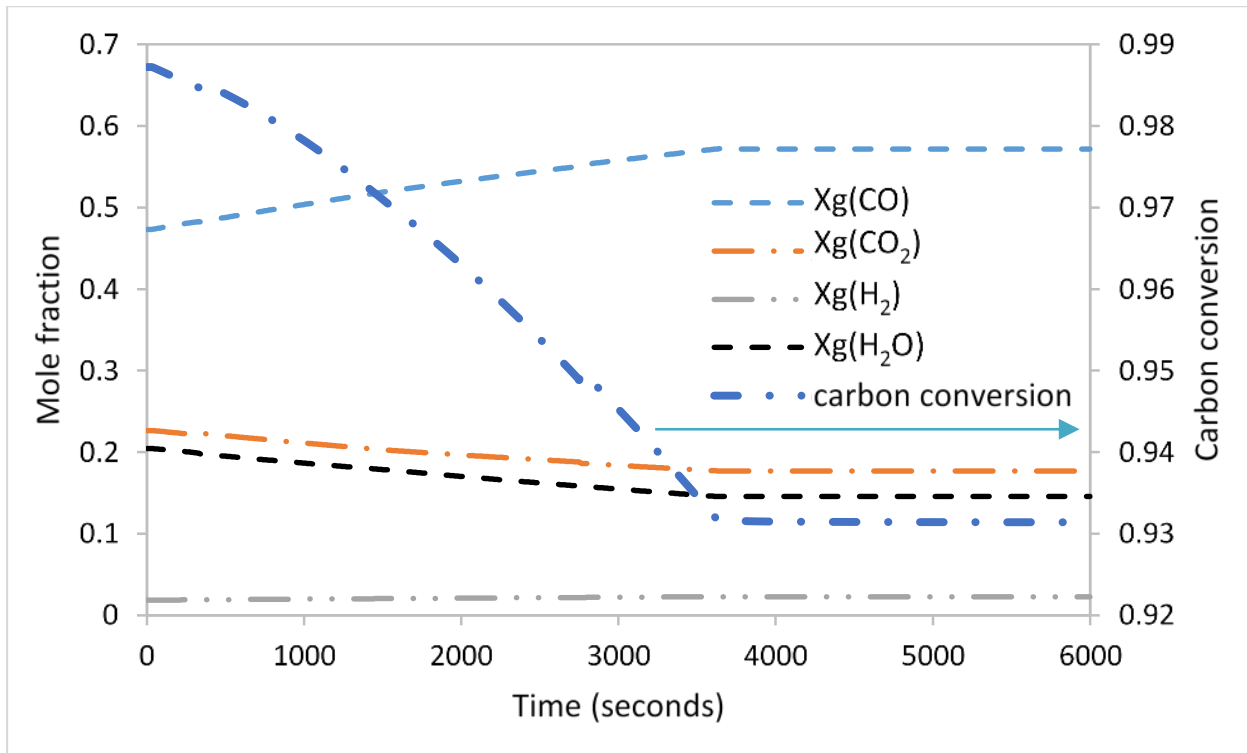

Figure 4.15: Change in outlet gas composition and carbon conversion during coal switch from Illinois \#6 to Pittsburgh \#8 coal

Figure 4.16 shows the trend of char and slag droplet deposition fluxes as well as the slag layer thickness profile before and after the coal switch from Illinois \#6 to Pittsburgh \#8. The drop in conversion means that less ash is being separated from the char particle and this ash is depositing on the slag layer with the deposition velocity of the char particles. A decrease in the slag droplet deposition flux is due to a decrease in the number density of slag droplets in all the bins and the decrease in ash content of Pittsburgh \#8 coal as can be seen from Table 4.2. 


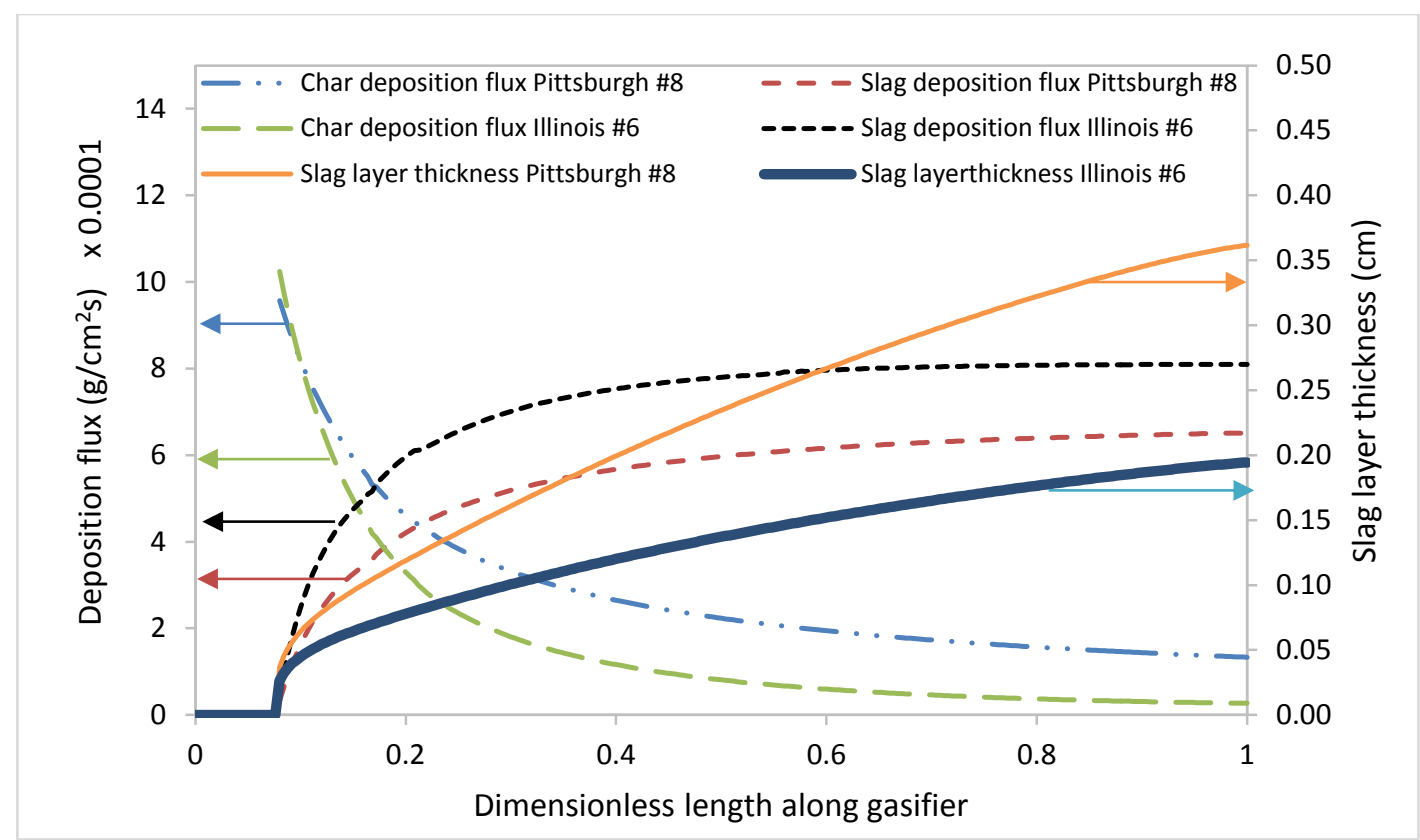

Figure 4.16: Deposition flux before and after change of coal from Illinois \#6 to Pittsburgh \#8

Although the net deposition flux decreases by 5\% after the coal switch, the slag layer thickness increases significantly. Figure 4.17 shows the slag temperature profile and the viscosity profile along the gasifier before and after the change has been implemented. For Pittsburgh \#8 coal ash, the fluid temperature is found to be around $1600 \mathrm{~K}$ in a reducing environment (Vaysman et al., 2012). It can be seen that the slag temperature approaches this temperature at the end of the coal switch. Due to the large decrease in slag layer temperature, and the change in the ash composition as the coals are switched, the viscosity increases significantly. 


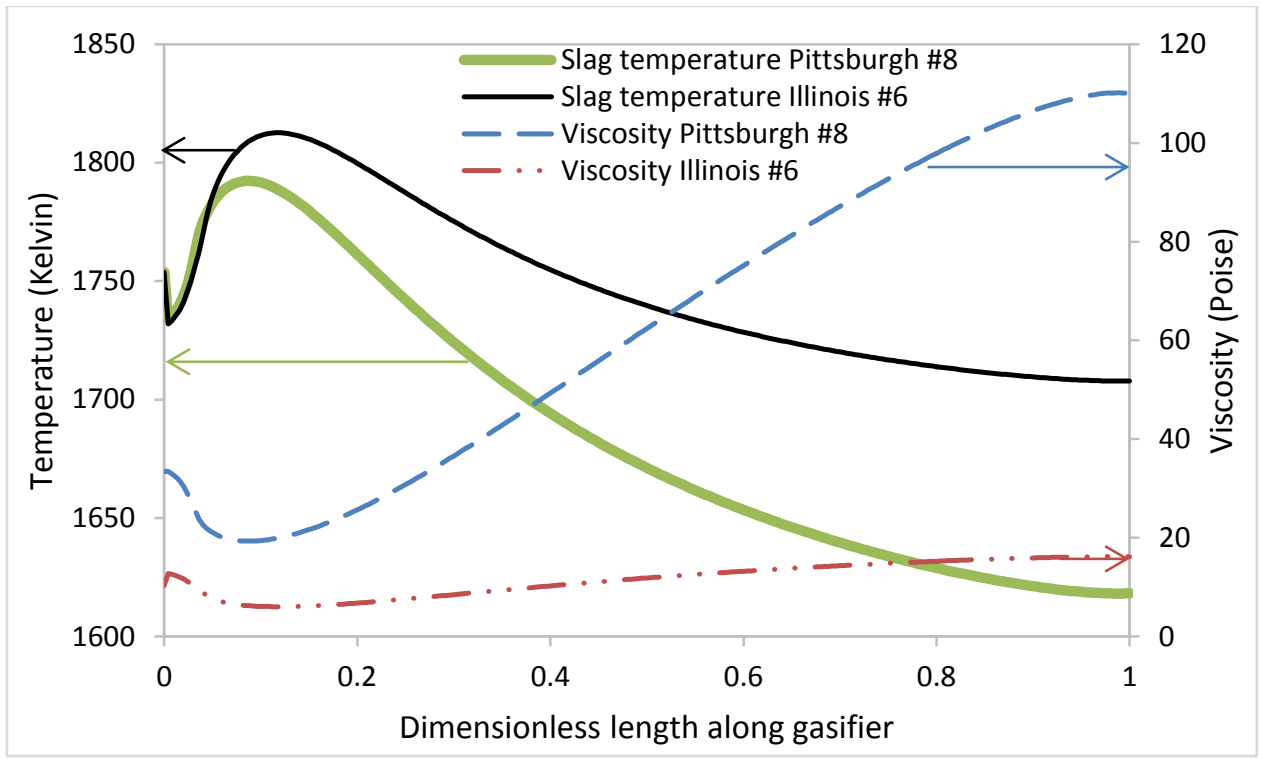

Figure 4.17: Slag layer temperature and viscosity profile before and after the coal switch

Manipulating the $\mathrm{O}_{2} /$ coal ratio can alleviate the issue of high slag layer thickness as observed before. The $\mathrm{O}_{2} /$ coal ratio can be changed to control the gasifier exit temperature or to control carbon conversion, if it can be estimated. Kasule et al. (2014) have implemented the later control strategy. Their work shows that the $\mathrm{O}_{2} /$ coal ratio for the Pittsburgh \#8 coal for same carbon conversion as the Illinois \#6 coal is about 0.9. For this $\mathrm{O}_{2} /$ coal ratio, the slag temperature at the exit of the gasifier is found to be around $1703 \mathrm{~K}$, which is slightly higher than that for the Illinois \#6 coal. The slag layer thickness for these conditions reduces to $0.25 \mathrm{~mm}$. 


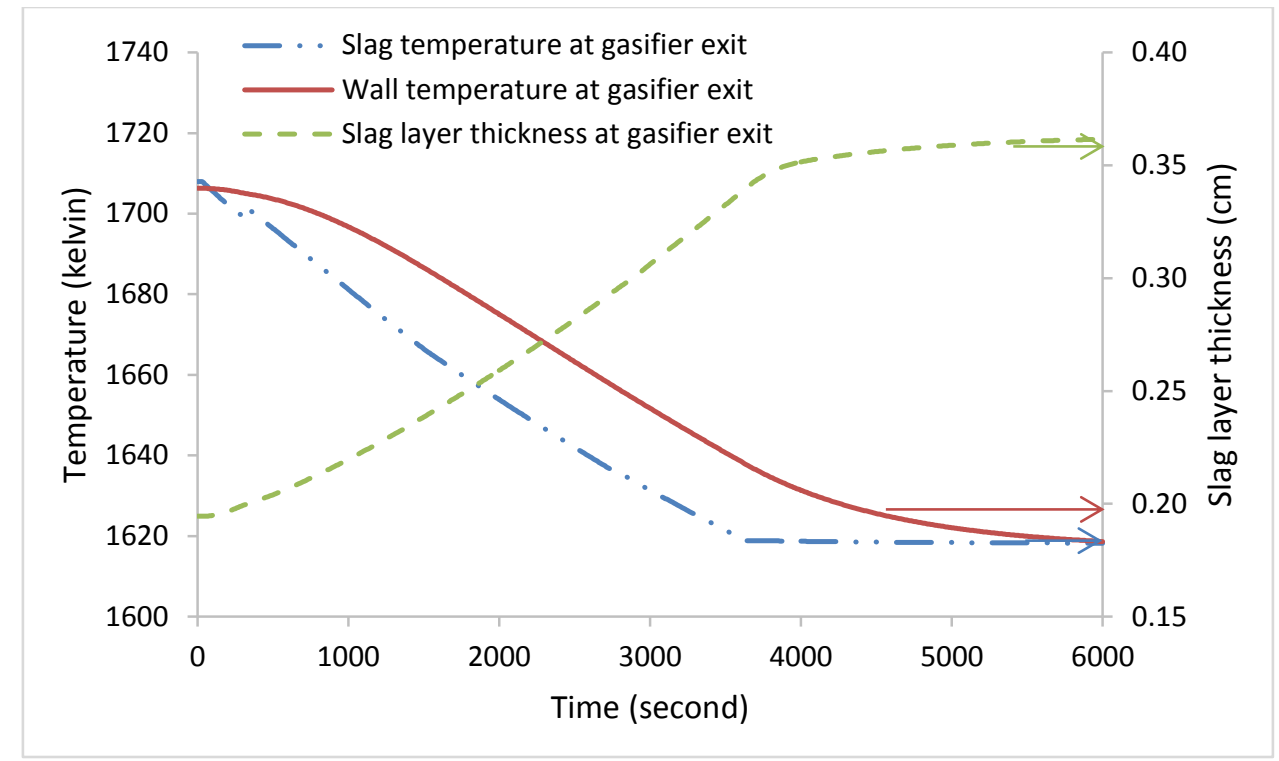

Figure 4.18: Transient response of slag layer and wall temperature and slag thickness at gasifier exit

An interesting difference between the temperature time scales of the slag layer and the wall is observed in Figure 4.18 using the base case $\mathrm{O}_{2} /$ coal ratio. Even though the final wall and slag layer temperatures are similar, the dynamics of the slag temperature is faster. As mentioned earlier, the deposition flux of slag to the wall remains almost the same. Therefore, the increase in slag layer thickness is mainly due to the decrease in slag viscosity. Therefore, the slag layer thickness dynamics have a similar time constant as the slag temperature dynamics. 


\subsection{Conclusions}

A dynamic slagging gasifier model with models of slag transport, deposition, and slag layer has been developed. The model takes into account the deposition flux of both char particles and slag droplets and how that affects the thickness of the slag layer as the operating conditions are changed.

For the base case conditions, the slag deposition is found to be about $2 \%$ of the inlet solid mass and the thickness of the slag layer is about $3 \mathrm{~mm}$. It is observed that the char impaction is the dominant mode of slag deposition in the early region of the gasifier whereas slag droplet deposition becomes dominant in the later section of the gasifier. The model shows that even if the PSD for slag droplets differ widely, the slag layer thickness does not change appreciably.

The model is also able to capture the effects of changes in gasifier operating conditions on the slag flow layer. It was found that changes in the $\mathrm{O}_{2} /$ coal ratio and silica ratio have the strongest effect on the slag layer thickness. When the $\mathrm{O}_{2} /$ coal ratio is changed from 0.79 to 0.85 , it is observed that the carbon conversion increases mainly due to an increase in the temperature that result in a decrease of the slag layer thickness by over $35 \%$. As the silica ratio is changed from 0.52 to 0.7 , the slag layer thickness can more than double. A change in the coal flow rate is found to have a smaller effect on the slag layer thickness.

During a coal switch from Illinois \#6 to Pittsburgh \#8 it was found that the slag layer thickness and the viscosity changed considerably when the $\mathrm{O}_{2}$ :coal ratio was left unchanged. The char deposition flux is found to increase while the slag deposition flux is found to decrease due to the coal switch. This is because less ash gets separated due to the decrease in conversion and because the ash content in Pittsburgh \#8 coal is lower than that of Illinois \#6 coal. It is observed that if the $\mathrm{O}_{2} /$ coal ratio is left unchanged, there is large decrease in slag layer temperature. In addition, the ash composition changes leading to significant increase in viscosity. However, if the $\mathrm{O}_{2} /$ coal ratio is changed to achieve the same carbon conversion as for the Illinois \#6 coal, the slag layer temperature remains much above the fluid temperature. The dynamics of the slag temperature is found to be faster than the dynamics of the wall temperature. The dynamics of the slag layer thickness are found to have a similar time constant as the slag temperature dynamics. 
Even though this study shows a high operating temperature can help to achieve low slag layer thickness and to achieve high carbon conversion, a high slag layer temperature also leads to faster slag penetration into the refractory brick accelerating refractory degradation. Thus optimal gasifier operating conditions should be obtained by taking these tradeoffs into consideration. 


\section{REFRACTORY DEGRADATION MODELING}

\subsection{Introduction}

It is difficult to measure the extent of refractory degradation using state of the art sensors due to the harsh operating conditions inside the gasifier. Refractory thickness and residual stress in the refractory layer are important variables that, if known, would be helpful in avoiding unexpected shutdown and in extending the refractory life resulting in improved availability of the IGCC plant.

The gasifier refractory is made up of several layers. The innermost layer is the high chrome layer. Refractory containing up to $95 \%$ chrome is used in this layer. The purpose of the layer is to withstand thermal shocks, attrition, and corrosion due to the slag layer. The following layer is the castable alumina layer. This layer provides a second layer of defense against corrosion. The third layer consists of silica bricks that act as insulation. The final layer is the metal shell of the gasifier unit.

A refractory model is developed in this work that calculates the temperature across various layers of the refractory and the concentration profile of the slag in the high chrome layer. Due to the large difference in time scale, it is difficult and intractable to solve the gasifier model together with the refractory degradation model. A one-way coupling is done assuming that the effect of the degradation on the gasifier operating variables is negligible. The gasifier model with slag flow is solved to obtain the variables including slag layer thickness on the wall and wall temperature. These variables are used as the boundary conditions for the degradation model.

The refractory model is used to develop a degradation model where compressive spalling is considered as the mechanism for refractory degradation. A thermo-elastic model is also developed to calculate the stresses in the refractory layer due to slag penetration and swelling of the refractory and due to the thermal and mechanical effects. 


\subsection{Refractory degradation - compressive spall modeling}

Compressive spalling takes place due to the swelling of the slag penetrated region in the refractory. When the slag penetrates the refractory, some of the $\mathrm{Cr}^{3+}$ ions in the refractory matrix get replaced by the $\mathrm{Fe}^{3+}$ ions present in the slag. The $\mathrm{Fe}^{3+}$ ions occupy a greater volume and the slag penetrated regions begin to swell. As the slag keeps penetrating into the refractory, the affected region increases and begins to buckle irreversibly. Finally, when the buckle is too large to be held together, it breaks and a spall occurs.

In order to simulate the process of slag penetration into the refractory, a dynamic model would be required. As the slag penetrates deeper into the refractory, the rate of penetration will change due to the decreasing temperature and/or change in gasifier operating conditions. For identifying the location in the gasifier where rate of slag penetration would be the fastest under nominal operating conditions, a 2D model of the wall is developed for calculating the concentration and temperature profile in the refractory is developed. The heat balance equation is written for all layers. The concentration equation is solved only for the first layer, viz. the high chrome layer as this layer is the most susceptible to spalling due to slag penetration. The stress models are applied to this identified location.

\subsubsection{Refractory model with slag penetration}

The thickness of the high chromia, alumina, silica and metal layers are considered to be $12 \mathrm{~cm}$, $10 \mathrm{~cm}, 10 \mathrm{~cm}$ and $5 \mathrm{~cm}$, respectively and is the same as used in the gasifier model (Robinson and Luyben, 2008).

The heat balance equation is given by Eq. 5.1.

$$
\rho_{c h} C_{p, c h} \frac{d T_{c h}}{d t}=k_{c h}\left[\frac{1}{r} \frac{d}{d r}\left(r \frac{d T_{c h}}{d r}\right)+\frac{d^{2} T_{c h}}{d z^{2}}\right]
$$

Here, $\rho_{c h}$ is the density of the chrome layer, $C_{\mathrm{p}, \mathrm{ch}}$ is the specific heat, $k_{\mathrm{ch}}$ is the thermal conductivity and $T_{\mathrm{ch}}$ is the temperature in the chrome layer. Equations similar to Eq. 5.1 are 
written for the remaining three layers with interface conditions of continuous temperature and heat flux. The heat loss from the final layer is calculated using Eq. 5.2:

$$
q_{\text {loss }}=h_{\text {air }}\left(\frac{\pi D_{o}}{4} \Delta x\right)\left(T_{m t, e n d}-T_{a m b}\right)
$$

where, $h_{\text {air }}$ is the heat transfer coefficient of air, $T_{m t, e n d}$ is the metal temperature in contact with the ambient air and $T_{a m b}$ is the ambient air temperature, taken as $20^{\circ} \mathrm{C}$. For the boundary for the heat equation at the top and bottom of the gasifier wall, it is assumed that the flux is zero for all of the layers.

The concentration is calculated as the percentage of pore volume occupied by slag. If the hot face is covered by slag, the concentration at the boundary is set as unity. To calculate the concentration profile of the slag in the refractory, the equation used is given in Eq. 5.3.

$$
\frac{d C_{\text {slag }}}{d t}=D_{e f f}\left[\frac{1}{r} \frac{d}{d r}\left(r \frac{d C_{\text {slag }}}{d r}\right)+\frac{d^{2} C_{\text {slag }}}{d z^{2}}\right]
$$

Here, $C_{\text {slag }}$ is the fraction of the pore occupied by slag and $D_{\text {eff }}$ is the diffusivity of slag in the high chrome layer. The diffusivity is a function of temperature and the equation for diffusivity used in this work is shown in Eq. 5.4 and is obtained by fitting data from Williford et al (2008).

$$
D_{\text {eff }}=1.446 \times 10^{-17} \exp \left(0.01468 T_{c h}\right)
$$

Using Eq. 5.4, the diffusivity through the high chromia layer is shown in Figure 5.1. Due to the decrease in the temperature along the wall, there is significant decrease in diffusivity suggesting that it is unlikely for the slag penetration front to move beyond the high chromia layer. 


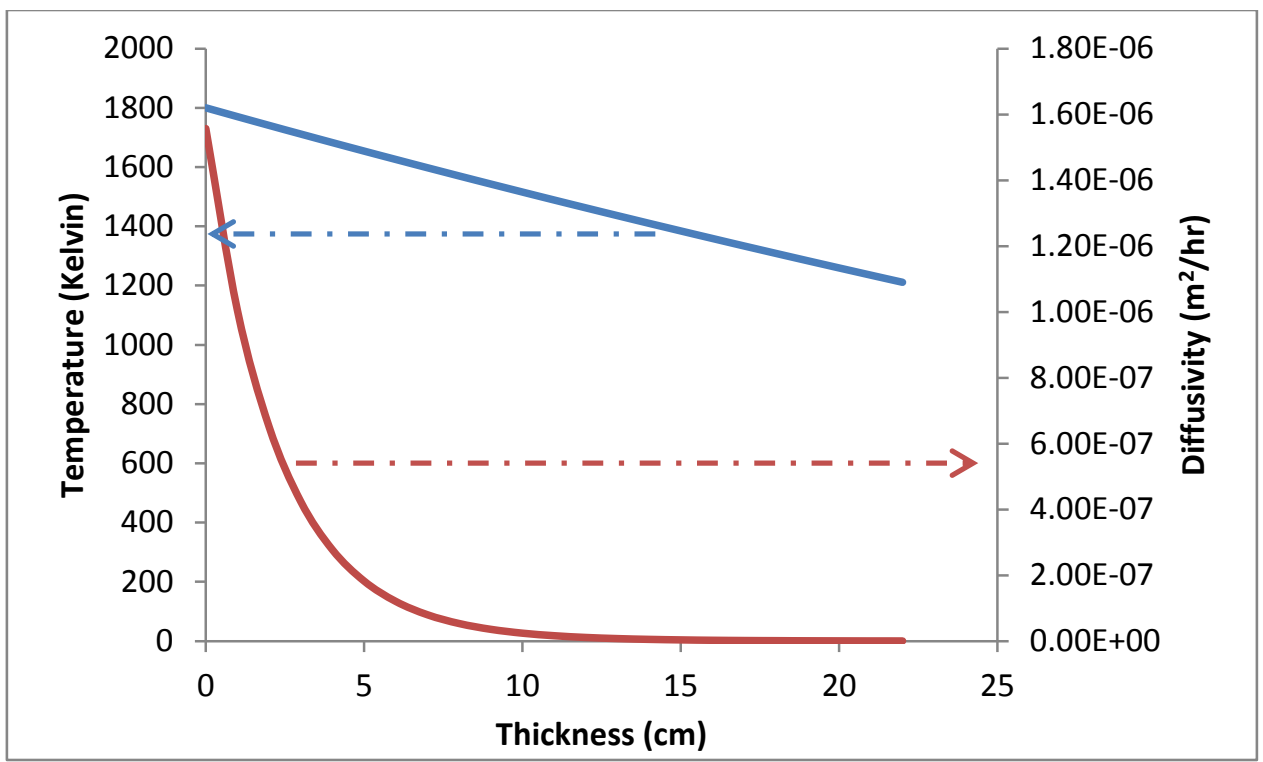

Figure 5.1: Temperature and diffusivity profile across the high chromia layer.

This model is able to capture the dynamic effect of slag penetration front as it slows down when penetrating deeper into the refractory brick.

\subsubsection{Compressive spalling model}

The compressive spalling model is developed mainly based on the work of Johnson et al. (2008) and Williford et al. (2008, 2010). Slag diffuses into the pores in the first layer affecting the refractory properties. Due to the difference of volume between the slag affected and the slag free refractory, and their thermal expansion coefficient, a strain is developed which causes the slag penetrated region to swell and separate in the form of a blister (Williford et al., 2008). The blister will continue to buckle and finally spall as the slag continues to penetrate after a certain depth.

The average slag penetration depth is taken at the location where the slag concentration is $30 \%$ (Johnson et al., 2008). The minimum distance required for spalling (Williford et al., 2008) is given by Eq. 5.5:

$$
h_{\min }=\frac{\Gamma \varphi^{2}\left(1-\vartheta_{p}\right)}{E \Delta \varepsilon_{\text {total }}{ }^{2}}
$$

where, $\Gamma$ is the toughness, $\varphi$ is a dimensionless parameter with a value of $2.5, \vartheta_{p}$ is the Poisson's ratio, E is the Young's modulus of the penetrated region and $\Delta \varepsilon_{\text {total }}$ is the total 
differential strain. The strain is calculated by combining swelling due to slag penetration as well as differential thermal expansion between the slag penetrated and slag free refractory:

$$
\Delta \varepsilon_{\text {total }}=\Delta \varepsilon_{g}+\Delta \varepsilon_{t h}
$$

where, $\Delta \varepsilon_{g}=\frac{\Delta V_{C r}}{3 V_{C r}}$ and $\Delta \varepsilon_{t h}=\Delta \alpha \Delta T$ in which $\Delta \alpha$ is the difference in thermal expansion coefficients between the slag-penetrated and slag-free refractory and $\Delta T$ is the temperature gradient in the refractory wall. The value of $\frac{\Delta V_{C r}}{V_{C r}}$ is set as 0.0032 based on the work of Williford et al. (2008).

Using the refractory model with slag concentration, and the criteria to calculate the mean penetration depth, the slag penetration front can be tracked as it moves through the refractory brick. When the slag penetration front reaches the minimum depth, calculated by Eq. 5.5, a spall is assumed to have occurred. 


\subsection{Refractory stress model}

A more detailed refractory degradation model is developed that can calculate the stress build-up in the refractory. In comparison to the state-of-the-art model presented before, following contributions have been made in this work:

- Analytical equations are derived to capture the effects of thermal and mechanical stresses and strain due to slag penetration on the stress in the refractory. This model provides the profile of radial stress, hoop stress, and longitudinal stress through all layers of the refractory. While the previous model assumes that the spall takes place only due to slag penetration and therefore, sets the strain to zero in absence of slag penetration, this model makes no such assumption and therefore can be used to calculate the stress profile due to change in the temperature profile and/or due to change in the refractory properties due to aging or degradation mechanisms other than slag penetration.

- Drawing from the classical theory of elasticity, this work has considered a failure criterion that is applicable for all cases where there is residual stress rather than considering buckling to be the mechanism for failure as in the previous model. It should be noted that while failure due to buckling when it exceeds some critical size is dominantly considered for a failure criteria for metals, it is unlikely for refractory materials that are brittle and cannot sustain plastic deformation. Furthermore, the work of Williford et al. (2008) have drawn the theory from a work that has focused on laminated metal sheets where buckling would led to delamination (He et al., 1998). This phenomenon is unlikely to occur for the composite wall made out of refractories. Therefore, the theory and approach developed in this work can not only be used for calculating thermal and mechanical stresses in the slag-free refractory wall, it is expected to be more realistic representation of the refractory wall in presence of slag penetration.

Such a model, to the best of the author's knowledge, is not available in the literature. 


\subsubsection{Thermo-elastic stress model}

Several assumptions have been made in the model and have been listed below:

1. Stress relaxation due to creep is not considered.

2. A 1-D model is considered in this work. Therefore, variation of stresses in the theta direction is neglected.

3. Plane strain is assumed as a simplification where the material is restricted to expand in the axial direction at the selected location. This assumption is made to calculate the maximum stress that can be generated at a location. The actual stress is expected to be between the limiting cases of plane strain (zero axial strain) and plane stress (zero axial stress, free to expand axially).

4. The axial stress can be relieved using spring loaded or other flexible structures. Therefore, the axial stress is not considered as a candidate stress that could lead to failure. In this study, the radial and hoop stresses are discussed.

5. The stress model is developed for the high chrome, alumina and silica layer. Due to the very high thermal conductivity of the metal layer and relatively low temperature as well lower mechanical stress at that boundary, metal layer is not considered.

6. The shear stresses are neglected.

The analytical equations for stresses in the radial, hoop and axial direction are derived using the stress-strain equations, strain-displacement relations and the stress equation for radial coordinates. At equilibrium, Eq. 5.7 is satisfied based on Assumption 3.

$$
\frac{d \sigma_{r r}}{d r}+\frac{\sigma_{r r}-\sigma_{\theta \theta}}{r}=0
$$

Here, $\sigma_{r r}$ is the radial stress in the radial direction and $\sigma_{\theta \theta}$ is the hoop stress.

The strain equation is obtained by considering the strain developed due to the mechanical, thermal and slag penetration effects. The strains in the radial and hoop directions are shown in Eq. 5.8-5.9 below. The axial strain is assumed to be zero.

$$
\varepsilon_{r r}=\frac{1}{E}\left[\sigma_{r r}-\vartheta\left(\sigma_{\theta \theta}+\sigma_{z z}\right)\right]+\alpha \Delta T+k_{s l}
$$




$$
\varepsilon_{\theta \theta}=\frac{1}{E}\left[\sigma_{\theta \theta}-\vartheta\left(\sigma_{r r}+\sigma_{z z}\right)\right]+\alpha \Delta T+k_{s l}
$$

The stresses in one dimension cause a strain in the other dimensions. This change in strain is different by a factor of $\vartheta$, which is the Poisson's ratio. The effect of thermal expansion is calculated in terms of the reference condition, which is taken to be room temperature. An additional term $k_{s l}$ is considered in this work that captures the effect of the change in strain due to the swelling of the slag penetrated region. This term is calculated by assuming that the strain is proportional to the concentration of slag in the pores and that it changes linearly with respect to the maximum swelling obtained from Williford et al. (2008).

The equation to calculate $k_{s l}$ is the shown in Eq. 5.10.

$$
k_{s l}=C_{\text {slag }} \frac{\Delta V}{3 V}
$$

$C_{\text {slag }}$ is obtained from the slag concentration equations and varies from 0 to 1.

The strain-displacement equations in the cylindrical coordinates for the radial and hoop strains are given as Eq. 5.11-5.12.

$$
\begin{aligned}
& \varepsilon_{r}=\frac{d u}{d r} \\
& \varepsilon_{\theta}=\frac{u}{r}
\end{aligned}
$$

Here, $u$ is the expansion in the radial direction.

The stress equations Eq. 5.8-5.9 can be rearranged in terms of stress to obtain an equation for the radial and hoop stress shown in Eq. 5.13-5.14 below.

$$
\begin{gathered}
\sigma_{r r}=\frac{E}{(1+\vartheta)(1-2 \vartheta)}\left[(1-\vartheta) \varepsilon_{r}+\vartheta \varepsilon_{\theta}-(1+\vartheta) \alpha \Delta T-(1+\vartheta) k_{s l}\right] \\
\sigma_{\theta \theta}=\frac{E}{(1+\vartheta)(1-2 \vartheta)}\left[(1-\vartheta) \varepsilon_{\theta}+\vartheta \varepsilon_{r}-(1+\vartheta) \alpha \Delta T-(1+\vartheta) k_{s l}\right]
\end{gathered}
$$

The axial stress is calculated using Eq. 5.15 and depends on the stress in the radial and hoop direction and the expansion due to thermal and slag effects. 


$$
\sigma_{z z}=\left(\sigma_{r r}+\sigma_{\theta \theta}\right) \vartheta-E \alpha \Delta T-E k_{s l}
$$

In order to derive the radial and hoop stresses, boundary values for the radial stress are needed. These equations are shown in Eq. 5.16-5.17:

$$
\begin{aligned}
& \text { At } r=a, \sigma_{r r}=-P_{1} \\
& \text { At } r=b, \sigma_{r r}=-P_{2}
\end{aligned}
$$

where $a$ and $b$ are the internal and external radii of the first refractory layer. $P_{1}$ and $P_{2}$ are the stresses at the boundaries.

Using Eq. 5.10-5.15 and boundary conditions in Eq. 5.16-5.17, the analytical equations for the radial and hoop stress are obtained as shown in APPENDIX C. These equations for the high chrome layer are shown in Eq. 5.18-5.19.

$$
\begin{aligned}
& \sigma_{r r, c h}=\frac{E_{c h}}{r^{2}(1-v)} \cdot\left(\alpha_{1} \int_{a}^{r} \Delta T_{1} r d r+\int_{a}^{r} \mathrm{k}_{s l} r d r\right)+\frac{r^{2}-a^{2}}{b^{2}-a^{2}} \frac{E_{c h}}{r^{2}(1-v)} \cdot\left(\alpha_{1} \int_{a}^{b} \Delta T_{1} r d r+\int_{a}^{b} \mathrm{k}_{s l} r d r\right)+\frac{P_{1} a^{2}}{b^{2}-a^{2}}\left(1-\frac{b^{2}}{r^{2}}\right)- \\
& \frac{P_{2} b^{2}}{b^{2}-a^{2}}\left(1-\frac{a^{2}}{r^{2}}\right) \\
& \sigma_{\theta \theta, c h}=\frac{E_{c h}}{r^{2}(1-v)} \cdot\left(\alpha_{1} \int_{a}^{r} \Delta T_{1} r d r+\int_{a}^{r} \mathrm{k}_{s l} r d r\right)+\frac{r^{2}+a^{2}}{b^{2}-a^{2}} \frac{E_{c h}}{r^{2}(1-v)} \cdot\left(\alpha_{1} \int_{a}^{b} \Delta T_{1} r d r+\int_{a}^{b} \mathrm{k}_{s l} r d r\right)-E \alpha \Delta T_{1}-E . k_{s l}+ \\
& \frac{P_{1} a^{2}}{b^{2}-a^{2}}\left(1+\frac{b^{2}}{r^{2}}\right)-\frac{P_{2} b^{2}}{b^{2}-a^{2}}\left(1+\frac{a^{2}}{r^{2}}\right)
\end{aligned}
$$

Here, $\alpha_{1}$ is the thermal expansion coefficient of the high chrome layer, and $\Delta T_{1}$ is the temperature difference between the temperature of the control volume and the reference temperature, shown in Eq. 5.20.

$$
\Delta T_{1}=T_{i}-293
$$

Eq. 5.18 - 5.19, are the stress equations for a single layer, and can be written similarly for the other layers with the corresponding change in the boundary conditions. These are shown below in Figure 5.2. 


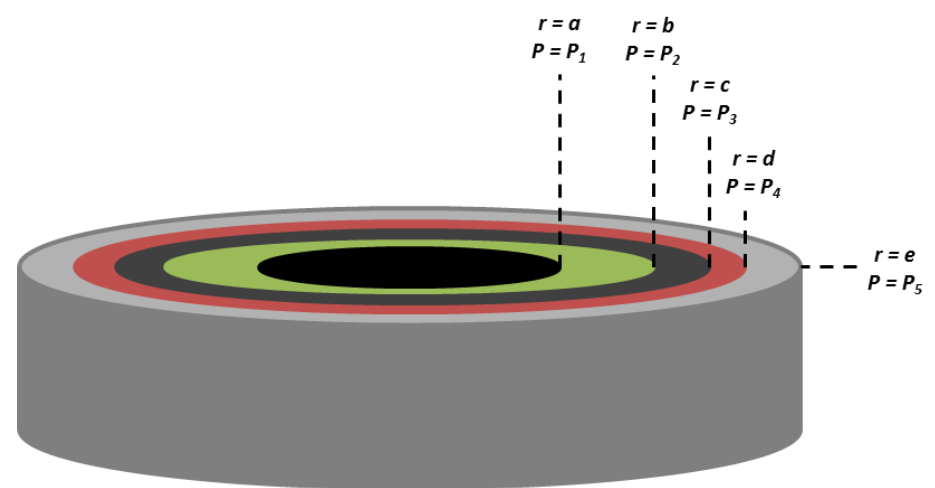

Figure 5.2: Boundary and interface conditions for stress in the multi-layer refractory wall.

At the interface, the stress and displacement are continuous. The interface conditions between the high chrome and alumina layers are shown in Eq. 5.21-5.23.

$$
\begin{aligned}
& \sigma_{r r, c h}(b)=-P_{2} \\
& \sigma_{r r, c h}(b)=\sigma_{r r, a l}(b) \\
& u_{c h}(b)=u_{a l}(b)
\end{aligned}
$$

Similar equations are written for the remaining interfaces. The parameter $u$ is calculated by rearranging Eq. 5.12 as shown in Eq. 5.24.

$$
u=\varepsilon_{\theta} r
$$

For the current work, no stress is assumed on the outer surface of the silica layer and is shown in Eq. 5.25.

$$
\sigma_{r r, s i}(d)=-P_{4}
$$

The material constants for the various layers are shown in Table 5.1. 
Table 5.1: Physical constants used in the stress model

\begin{tabular}{lcccc}
\hline \multicolumn{1}{c}{ Property } & High Chromia & Alumina & Silica & Metal \\
\hline Young's Modulus (MPa) & $4.13 \times 10^{10}$ & $1.10 \times 10^{10}$ & $6.50 \times 10^{9}$ & $2.00 \times 10^{11}$ \\
Poison's ratio & 0.3 & 0.3 & 0.165 & 0.25 \\
Thermal expansion $(\mathrm{mm} / \mathrm{mm} / \mathrm{K})$ & $7.68 \times 10^{-6}$ & $9.00 \times 10^{-6}$ & $6.30 \times 10^{-6}$ & $1.2 \times 10^{-5}$ \\
Density $\left(\mathrm{kg} / \mathrm{m}^{3}\right)$ & 4000 & 2760 & 2500 & 7800 \\
Specific heat $(\mathrm{kcal} / \mathrm{kg} / \mathrm{K})$ & 0.1784 & 0.220 & 0.250 & 0.120 \\
Thermal conductivity $(\mathrm{Kcal} / \mathrm{m} / \mathrm{hr} / \mathrm{K})$ & 2.063 & 1.401 & 0.871 & 46.42 \\
\hline
\end{tabular}

The concentration and heat balance equations in 1D are solved along with the stress model equations and are shown in Eq. 5.25-5.26, respectively.

$$
\begin{gathered}
\rho C_{p} \frac{d T}{d t}=k\left[\frac{1}{r} \frac{d}{d r}\left(r \frac{d T}{d r}\right)\right] \\
\frac{d C_{\text {slag }}}{d t}=D_{\text {eff }}\left[\frac{1}{r} \frac{d}{d r}\left(r \frac{d C_{\text {slag }}}{d r}\right)\right]
\end{gathered}
$$

Eq. 5.25 is solved for all four layers, while Eq. 5.26 is solved for the high chromia layer only.

\subsubsection{Failure}

The refractory bricks are brittle in nature and exhibit very little plastic deformation after the yield stress. In order to model failure in the refractory layers, a phenomenological failure criterion is used. For compressive stress, the cold compressive strength (CCS) of the material is used and for tensile stress, the modulus of rupture (MOR) is used as the failure criteria. The values for CCS and MOR for $92 \%$ high chromia used in this work are $234 \mathrm{MPa}$ and $64 \mathrm{MPa}$, respectively and are taken from McGee (1991, Oakridge National Lab). The region that exceeds the compressive or tensile failure criterion is considered to have yielded; however, it is still attached to the refractory. If a spall takes place, the affected region will be removed all together. The failure criterion provides an estimate of the size of the spall that can take place. 


\subsection{Results}

For the current results, the base case results for the slagging gasifier model are used.

\subsubsection{Slag penetration}

A snapshot of the penetration of slag into the high chrome refractory wall is shown in Figure 5.3.
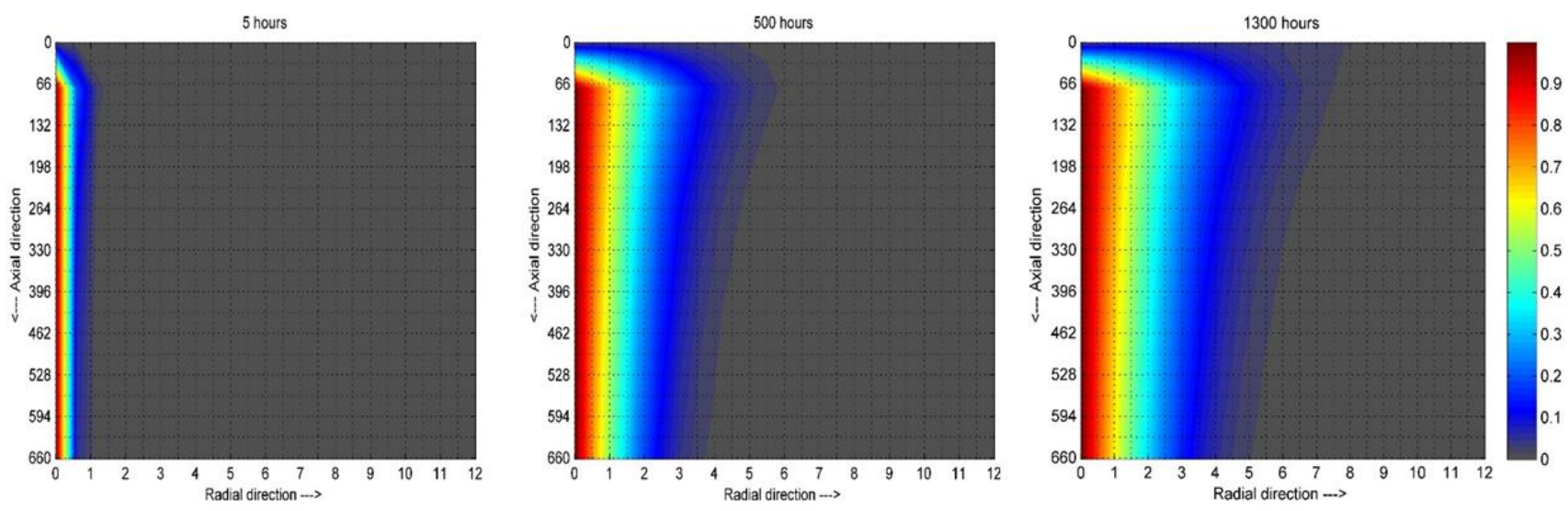

Figure 5.3: Slag penetration into the high chrome refractory at different time instances.

The high chrome layer on the gasifier wall is $660 \mathrm{~cm}$ in length and $12 \mathrm{~cm}$ in thickness. In the first $10 \%$ of the gasifier length, no slag is assumed to be on the wall of the refractory. After 500 hours, it can be seen that the slag penetration is highest at a distance of about $66 \mathrm{~cm}$ from the gasifier inlet. This is because, the wall temperature is highest at this location and diffusivity is a function of temperature. After significant penetration into the refractory however, the slag penetration rate in the radial direction decreases as the temperature reduces. Some diffusion in the axial direction is seen after this point.

The wall temperature at the location of maximum slag penetration is found to be $1800 \mathrm{~K}$. The steady state temperature profile of this axial location is shown in Figure 5.4. This location is considered for all studies presented hereafter. 


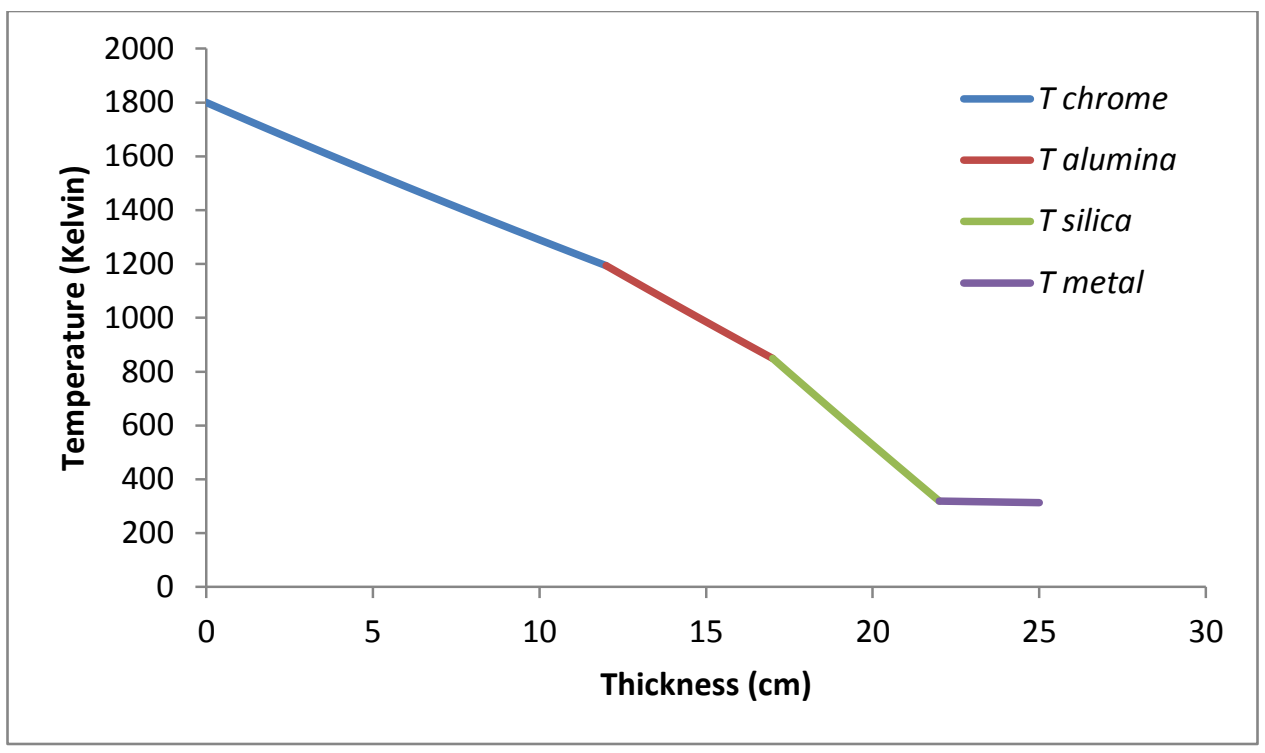

Figure 5.4: Steady state temperature profile along the refractory across the four layers.

\subsubsection{Compressive spall}

Four cases are simulated in the refractory degradation model. The first one is the base case where the gasifier is operated at a design inlet flow rate of $60,000 \mathrm{gm} / \mathrm{sec}$ of coal slurry. The second case is when the gasifier is subjected to feed oscillation leading to thermal cycling. A sinusoidal change in the gasifier feed with an amplitude of $10 \%$ of its base case value and a time period of 1 hr as shown in Figure 5.5 is considered in this work.

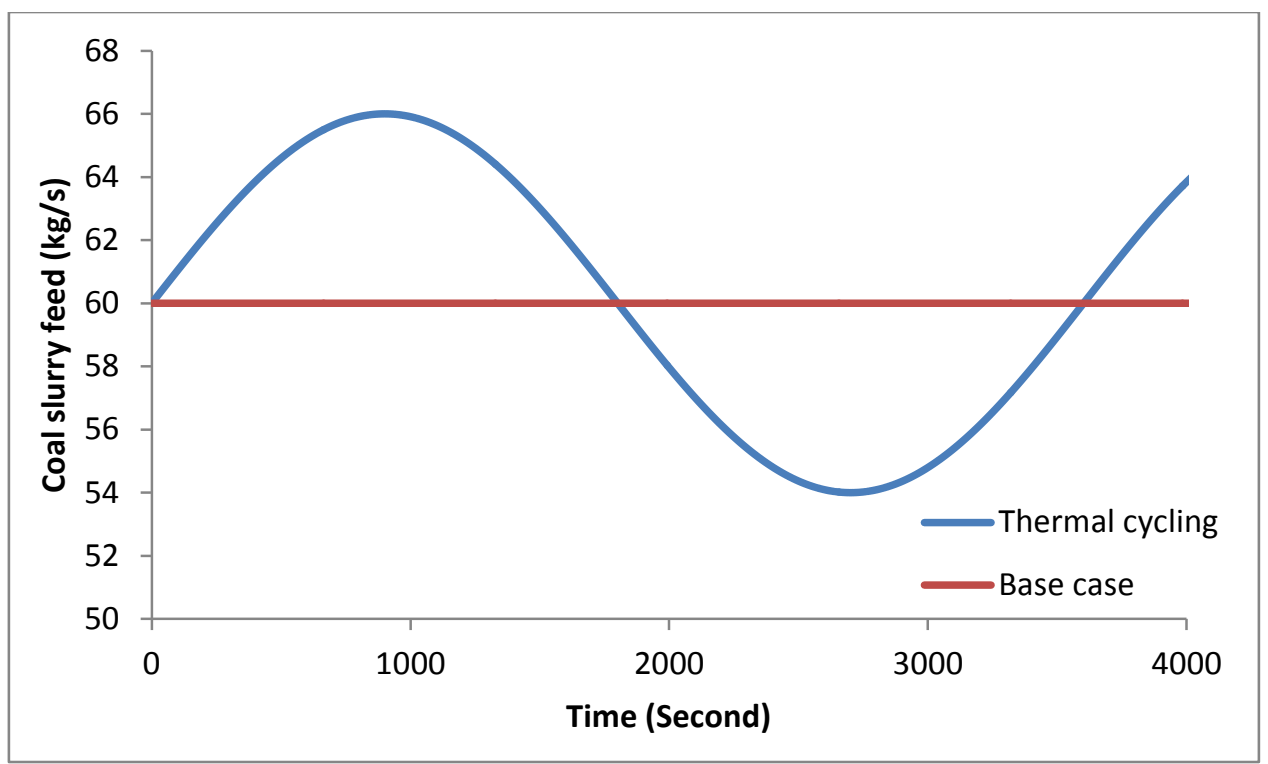

Figure 5.5: Coal slurry set point variation in the gasifier model 
The temperature variation at the same position is recorded for the two cases. The temperature profile for the oscillating coal feed is fit to a sinusoidal curve. The temporal temperature profiles are then used as an input to the degradation model. Two other case studies were also simulated where a high and low fixed temperature values were assumed as the hot face temperature. The high temperature value of $1850 \mathrm{~K}$ and low temperature value of $1775 \mathrm{~K}$ are used in these cases. The temperature profiles for the four cases used in the refractory degradation model are shown in Figure 5.6.

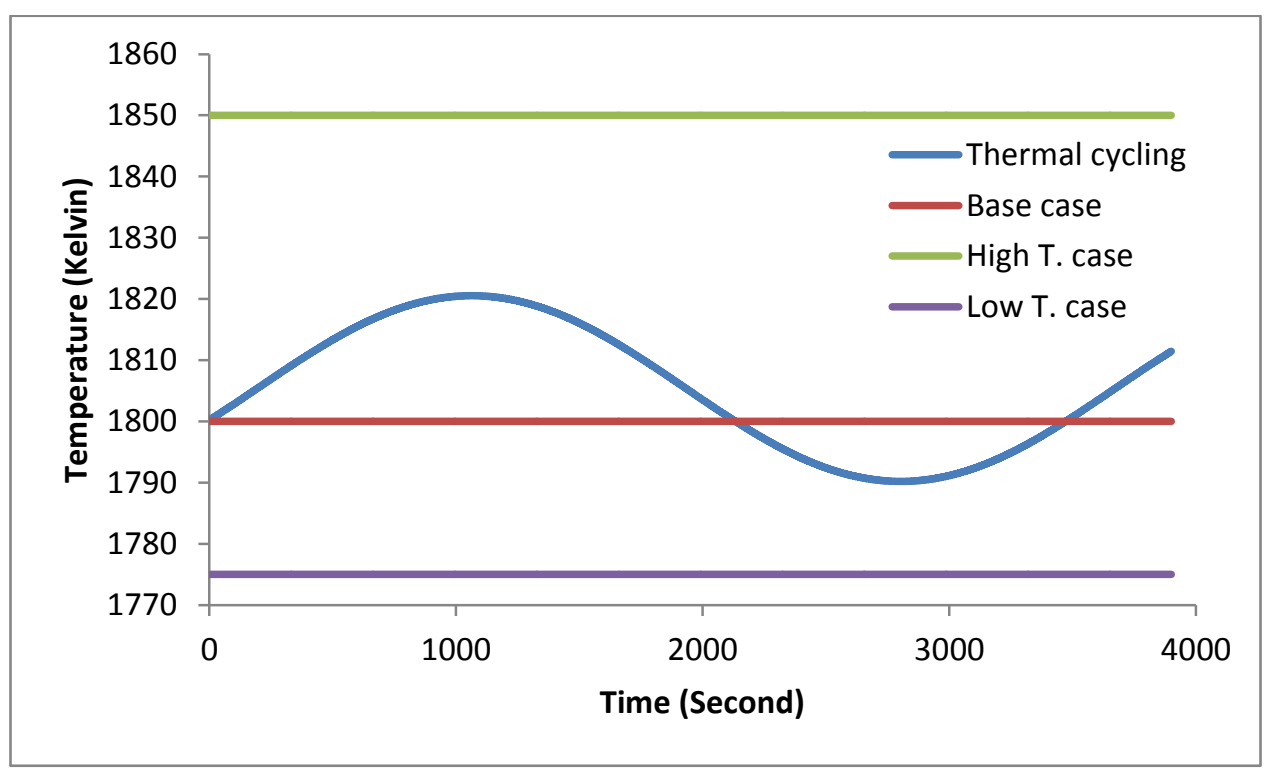

Figure 5.6: Wall temperature at the grid point selected for simulating degradation

From Figure 5.6 it can be seen that although in the second case the inlet flow rate of the coal slurry has been varied by $10 \%$, the wall temperature at the selected grid does not vary more than $15^{\circ} \mathrm{C}$ on average.

The refractory degradation model is simulated for all four cases and the results are plotted in Figure 5.7. 


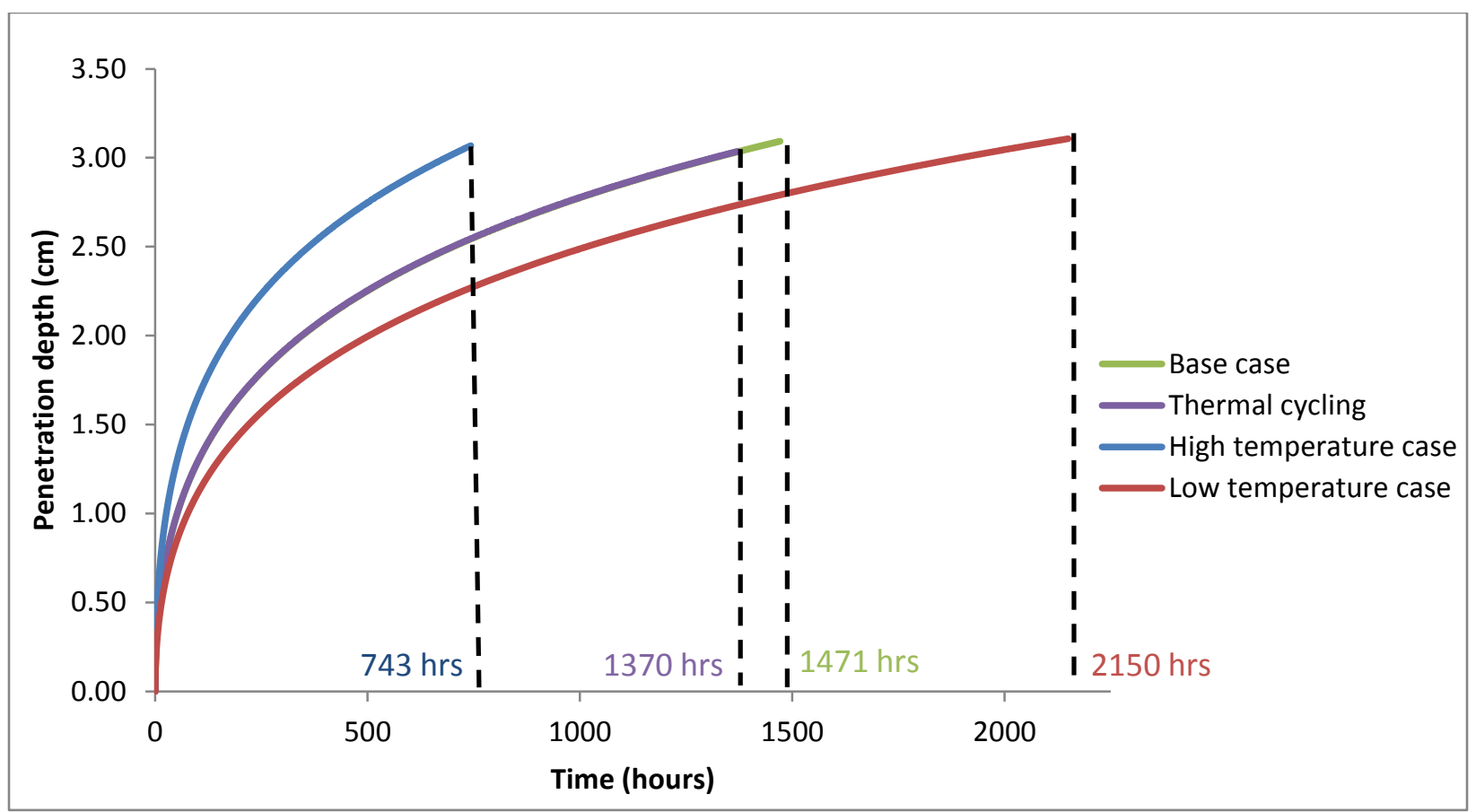

Figure 5.7: Time for first spall for the high temperature $(1850 \mathrm{~K})$, low temperature $(1775 \mathrm{~K})$, base case $(1800 \mathrm{~K})$ and thermal cycling case.

It can be seen that the time for the first spall to occur when the base case wall temperature is $1800 \mathrm{~K}$ is around $1471 \mathrm{hr}$. For the thermal cycling case, the curve seems to closely follow that of the base case but the first spall occurs around $100 \mathrm{hr}$ sooner. This could be because of a faster diffusion front. For the case of high temperature, spalling occurs at $743 \mathrm{hr}$. In the low temperature case, spalling occurs much later. However, due to its proximity to the critical viscosity temperature, such low temperature is avoided to prevent any solid slag build up on the wall of the refractory that could eventually lead to clogging of the gasifier. 


\subsubsection{Stress model results}

The stress model is solved for three layers of the refractory excluding the metal layer and the results for different cases are shown here.

\subsubsection{Stress profile for base case}

The radial profile for stress across the high chrome, alumina and silica layers is shown in Figure 5.8. No slag penetration is assumed in this case. Due to the elevated temperature, a thermal strain is developed. Due to the high Young's modulus, and the relatively high temperature of this layer, the maximum compressive stress occurs in this layer.

The interface pressure depends upon the stresses in the adjoining layers. The radial stress is continuous over the interface as can be seen in Figure 5.8. The results presented in Figure 5.8 are only for the thermal stress at steady state conditions.

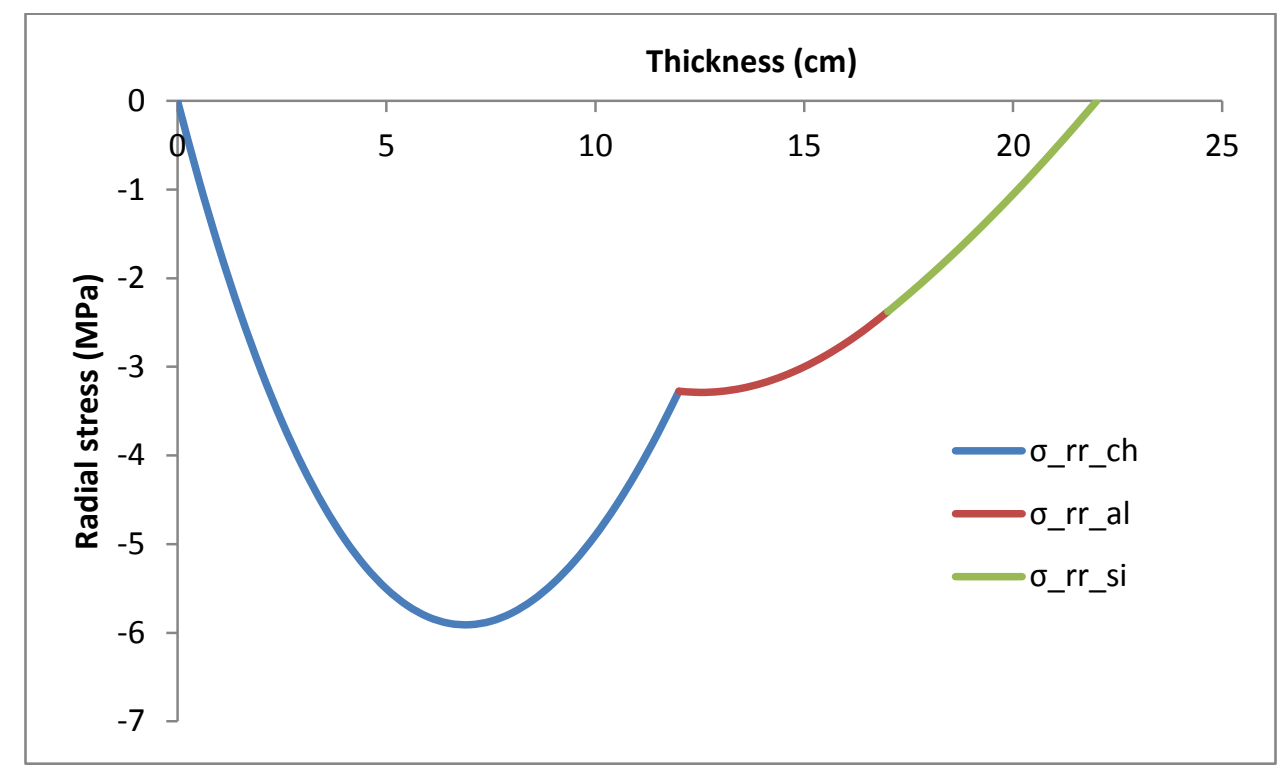

Figure 5.8: Radial stress profile across the high chrome, alumina and silica layers

The stress in the radial direction is compressive for all the layers. The maximum compressive radial stress in the high chrome layer appears to be much lower than the cold crushing strength at these high temperatures. 


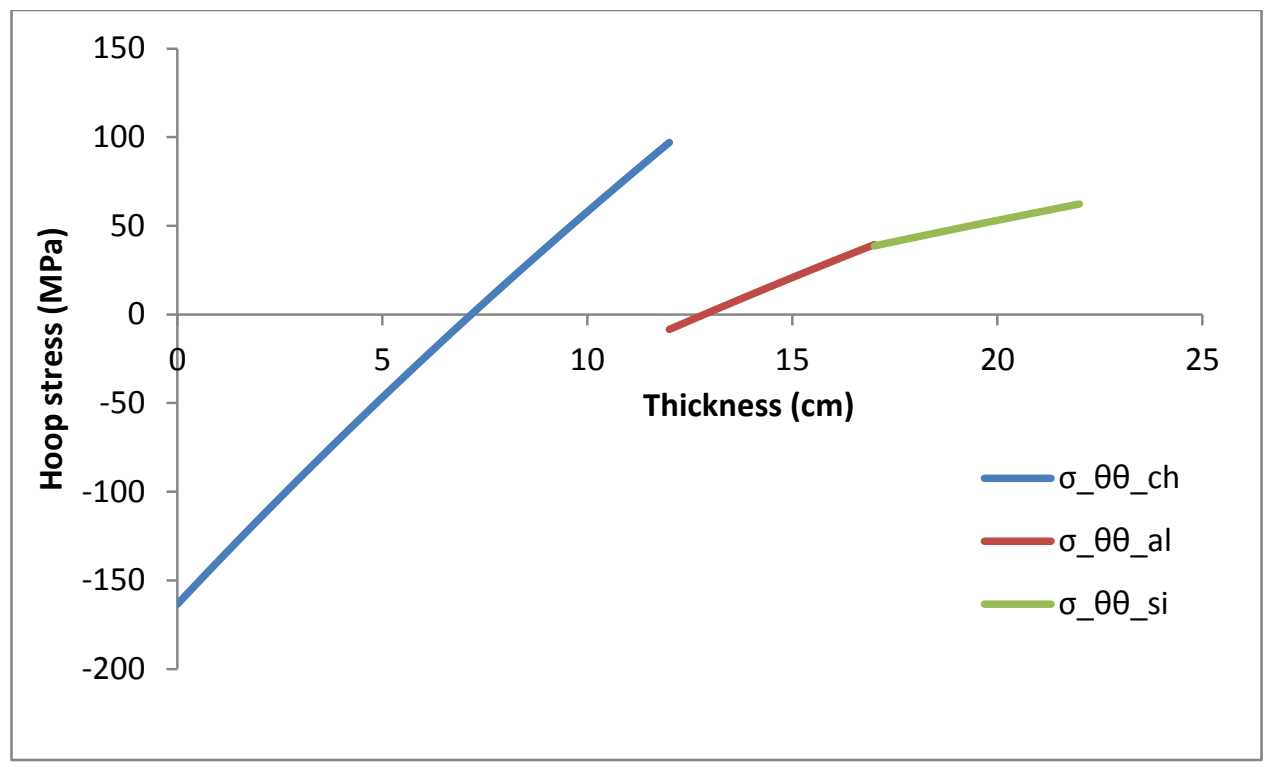

Figure 5.9: Hoop stress profile across the high chrome, alumina and silica layers

As shown in Figure 5.9, the hoop stress profile for the high chrome layer changes from compressive at the hot face, to tensile at the cold face. The alumina layer has a lower Young's modulus in comparison to the high chrome layer. Due to its higher thermal expansion coefficient, there is some compressive hoop stress at the hot side; however the layer is mostly in a tensile stress state in the hoop direction. The hoop stress is tensile in the silica layer as well.

At the hot face, the compressive stress is much higher than the maximum compressive radial stress in the high chrome layer but is still lower than the cold crushing strength of the material.

\subsubsection{Fault scenario simulation}

\subsection{Stress due to temperature effect}

The temperature of the hot side of the refractory layer is increased by ramping it up from $1800 \mathrm{~K}$ to $2000 \mathrm{~K}$ over a period of 100 hours. The results for the radial and hoop stress profile in the high chromia layer are shown in Figures 5.10 and 5.11. The temperature of the hot face is increased at a slow rate typical of the gasifier operation for avoiding thermal shock. 


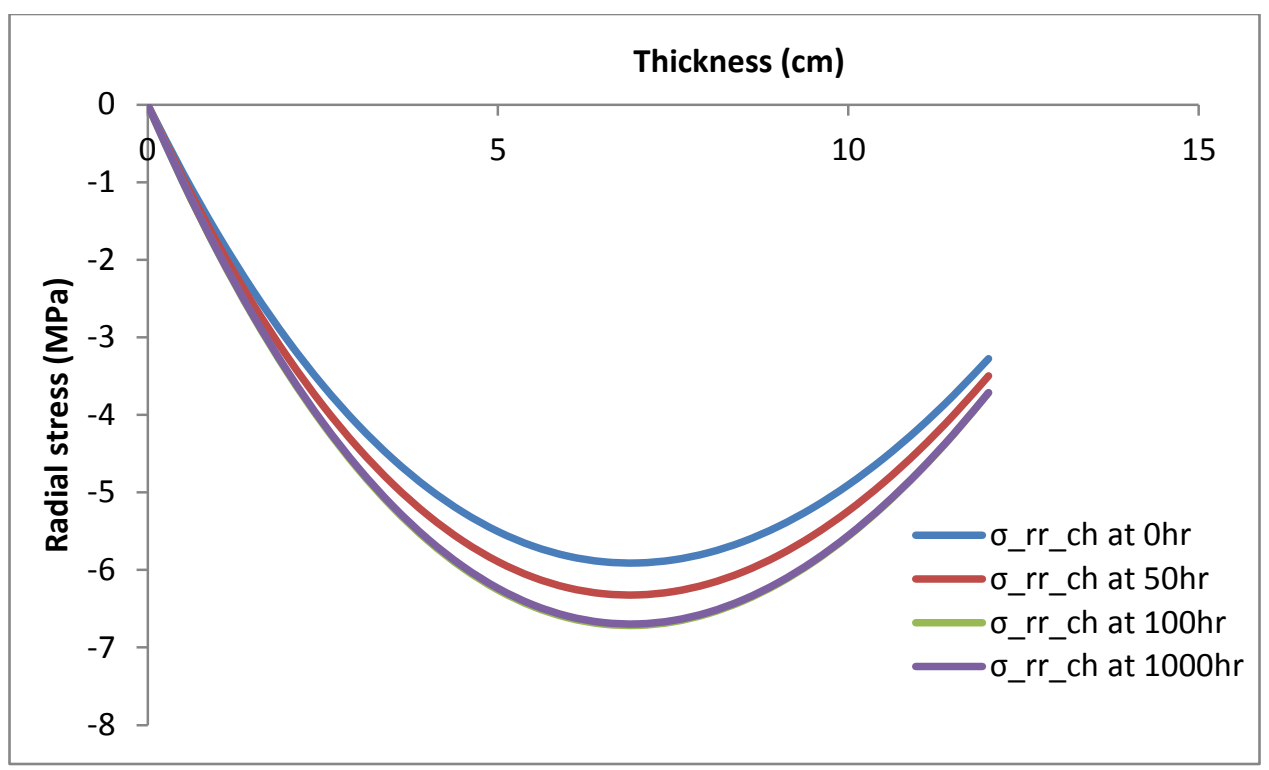

Figure 5.10: Effect of increase in temperature on the radial stress profile in the high chrome refractory

The radial stress profile becomes more compressive due to the increase in temperature. The maximum compressive radial stress increases the most in the chrome layer and in the same location as before. The interface stress also changes in response to the temperature increase. It can be seen that the stress response follows the temperature dynamics and does not change once the final temperature profile is reached. The hoop stress profile for the high chrome layer is shown in Figure 5.11. 


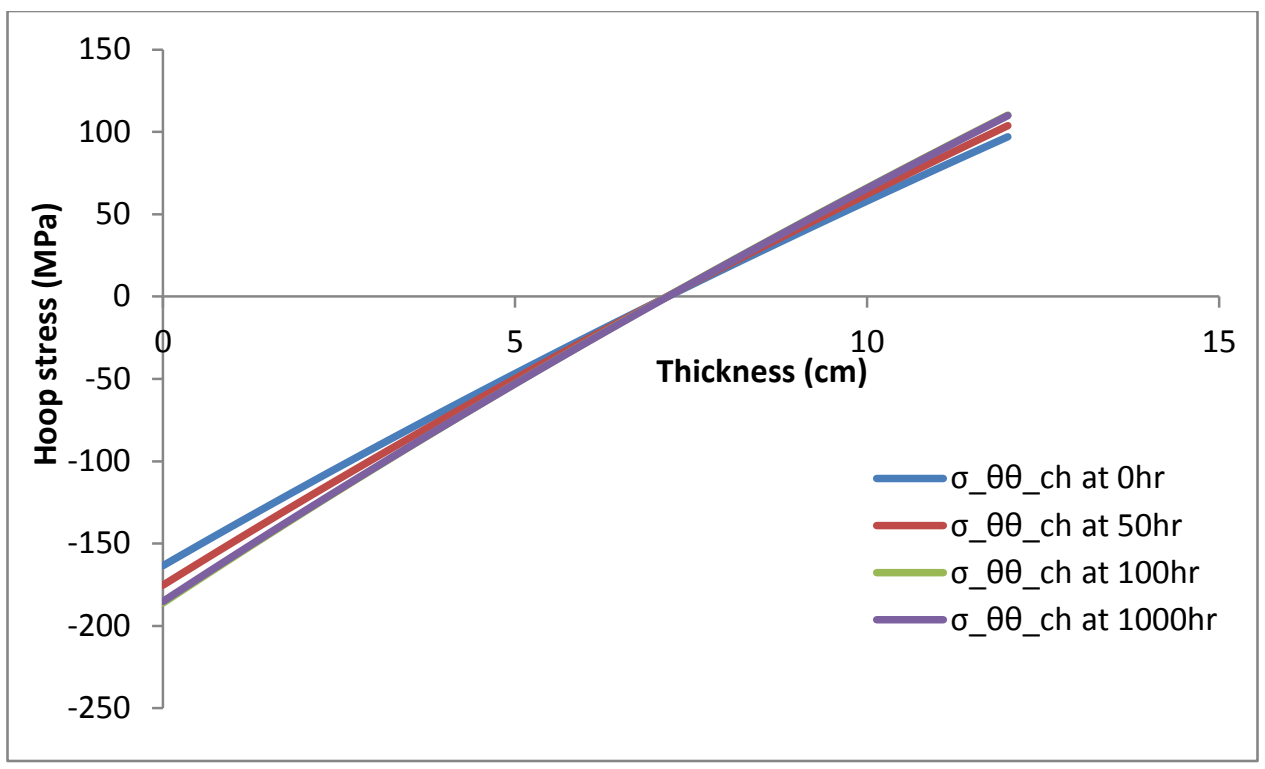

Figure 5.11: Effect of increase in temperature on the hoop stress profile in the high chrome refractory 


\subsection{Stress due to slag penetration}

The slag penetration profile into the high chrome refractory can be seen in Figure 5.12. As the slag penetrates deeper into the refractory, the rate of penetration decreases due to the temperature gradient. After 1000 hour, the slag reaches around $4 \mathrm{~cm}$ into the high chrome refractory brick.

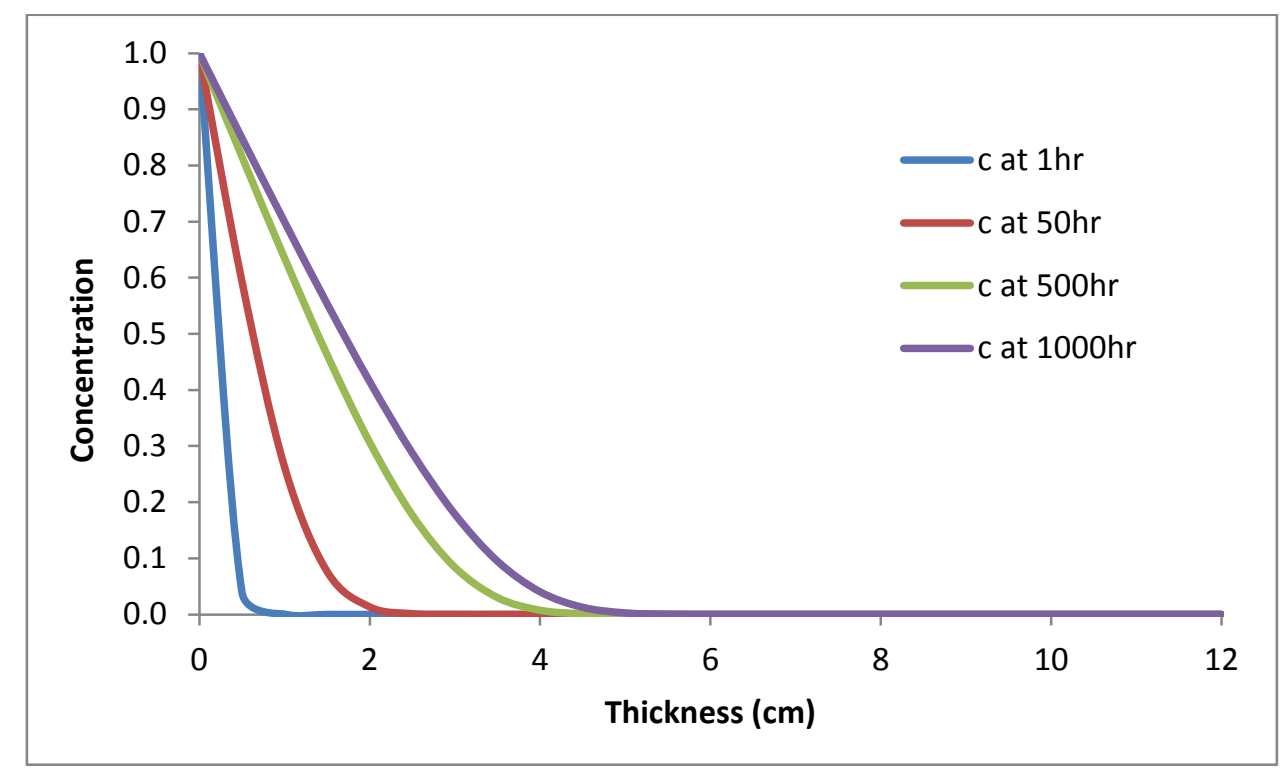

Figure 5.12: Slag penetration profile in the high chrome refractory layer with time

The effect of the slag penetration on the radial stress profile in the high chrome layer can be seen in Figure 5.13. The slag penetrated region begins to swell due to the exchange of $\mathrm{Fe}^{3+}$ and $\mathrm{Cr}^{3+}$ ions between the slag and the refractory. Although, from Figure 5.12 it can be seen that the slag penetration is taking place in the initial few centimeters of the chrome layer, the effect of the swelling results in an increase in the compressive stress in the entire layer. 


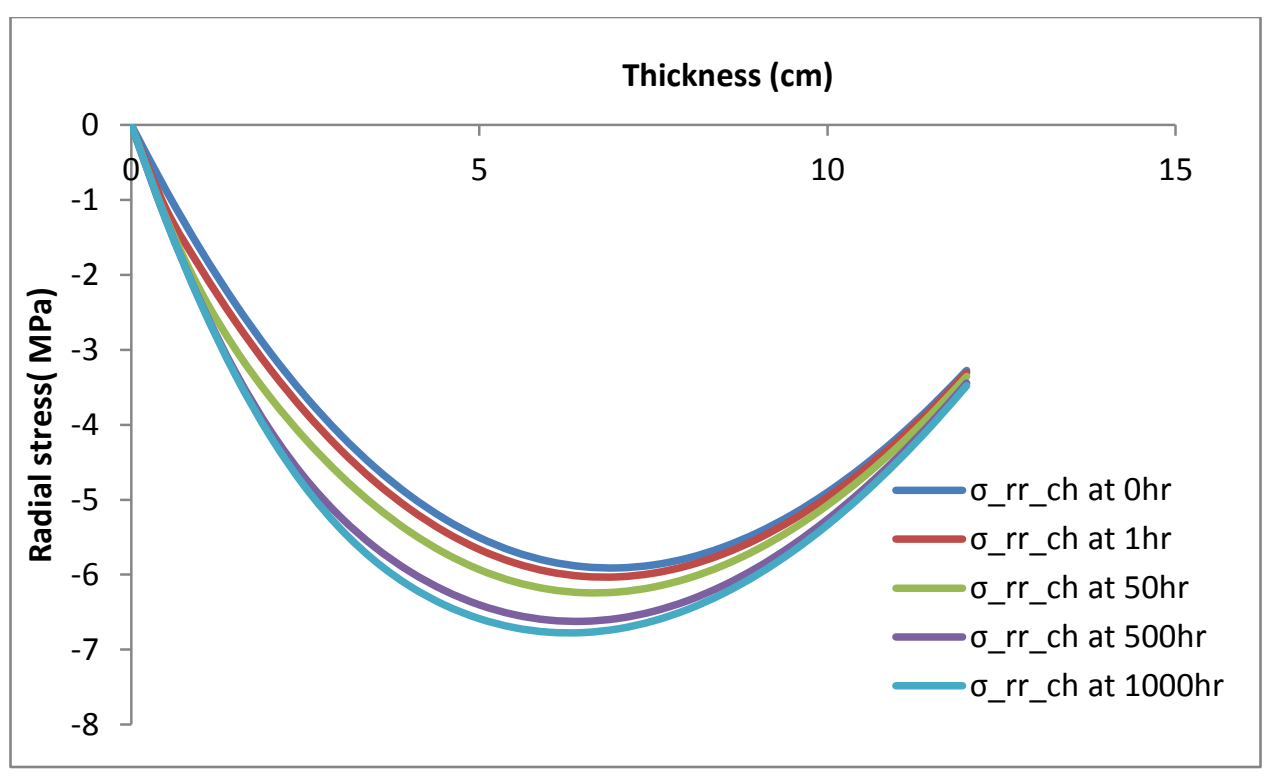

Figure 5.13: Temporal change in the radial stress profile of the high chrome refractory

The response of the hoop stress profile shown in Figure 5.14 is very different. The effect of the swelling appears to be more localized in the region affected by slag penetration.

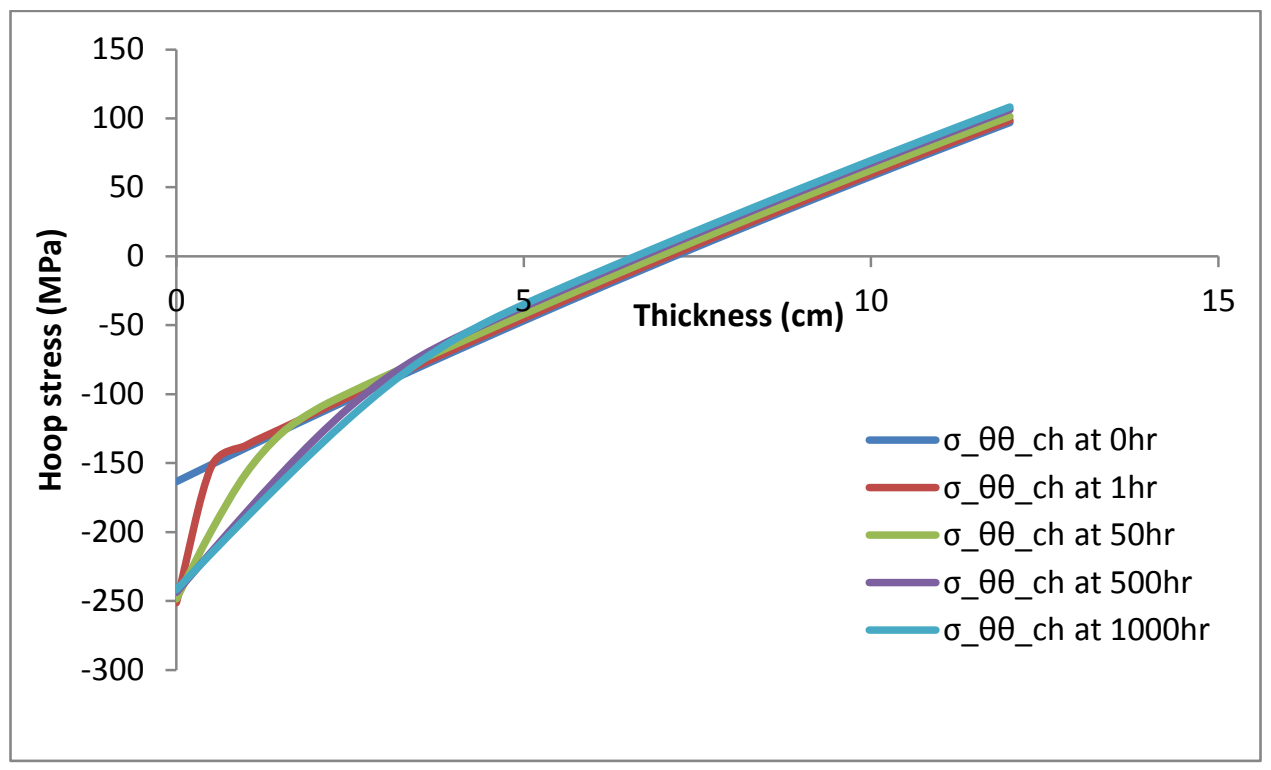

Figure 5.14: Temporal change in the hoop stress profile of the high chrome refractory 


\subsection{Conclusions}

A refractory degradation model is developed and implemented at the location where the temperature and slag penetration are the maximum in the gasifier. A compressive spalling model is developed to calculate the size and time for a spall to take place at this location. It is found that at base conditions, a spall can take place at about 1470 hour. A stress degradation model is also developed in this work that considers the effect of thermal and mechanical strains and the strain developed due to the swelling of the slag affected region of the refractory on the radial and hoop stress profiles. The present work also incorporates the mechanical strain and stress developed due to considering multiple concentric layers of the refractory and the effect of different material properties on the final stress profile. Several cases are simulated that show that the slag penetration has a strong effect on the change in stress profile. It shows that the slag penetration results in the yield of the hot face. 


\section{SWGSR MODELING}

\subsection{Introduction}

The SWGSR plays a key role in maintaining the desired $\mathrm{H}_{2} / \mathrm{CO}$ in the syngas being sent to the AGR plant. This unit usually consists of a two-stage reactor with an inter-stage cooler to obtain the desired conversion of CO. As described in Chapter 2, the sour water gas shift reactor catalyst is sulfur tolerant and also converts $\mathrm{COS}$ to $\mathrm{H}_{2} \mathrm{~S}$, that can be captured in the AGR.

However, the catalyst deactivation is one of the issues in this reactor. It is important to monitor the catalyst deactivation over time so as to operate the unit for longer life of the catalyst as well as plan for catalyst replacement and operate the plant accordingly.

A water gas shift reactor is simulated as part of the overall plant model (Bhattacharyya, 2010) using models from the Aspen Plus library, but that model cannot be used for simulating faults involving change in catalyst properties. Furthermore, a distributed model is required that can be used in order to determine the location of sensors so that spatial resolution of the faults can be obtained. The subsequent section discusses the modeling of a single stage sour water gas shift reactor in MATLAB, and the simulation of the catalyst deactivation fault. This model is then enhanced to a commercial-scale two-stage reactor system with an inter-stage cooler with additional models for faults.

\subsection{Model Development - Single Stage SWGSR}

The reactor is modeled as a plug-flow reactor. The conservation laws for mass, energy and momentum are derived accordingly. For this, radial variations of transport variables are neglected and the gradients are only considered in axial direction. $\mathrm{H}_{2}, \mathrm{CO}, \mathrm{CO}_{2}, \mathrm{H}_{2} \mathrm{~S}, \mathrm{H}_{2} \mathrm{O}$ and $\mathrm{COS}$ are considered in the species balance. Although gases such as $\mathrm{N}_{2}, \mathrm{Ar}$ and $\mathrm{O}_{2}$ may be present in the reactors in plants, the model equations can be extended easily to include these components, as they are present in very small quantities and do not react. The kinetic parameters 
for the COS hydrolysis are obtained from the work of Svoronos et al. (2002), whereas the rate parameters for the WGS reaction are obtained through data reconciliation considering a pseudofirst order reaction (Berispek, 1975). A schematic of the single stage SWGSR is shown in Figure 6.1.

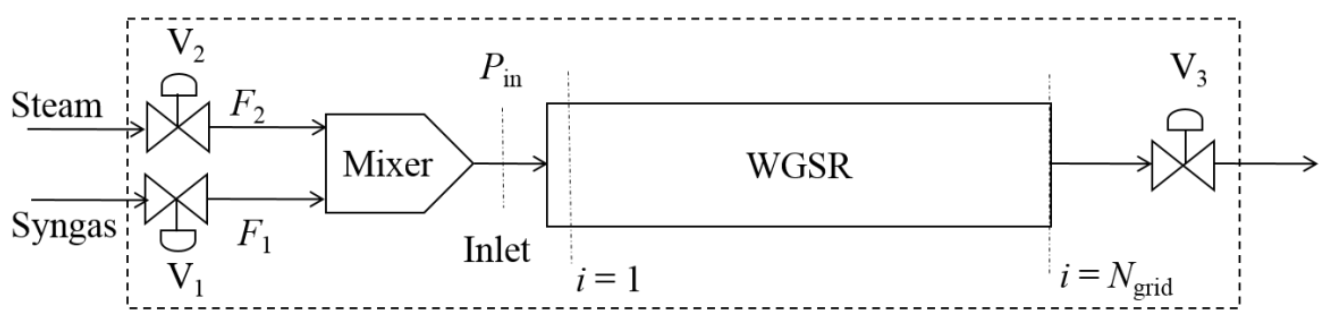

Figure 6.1: Schematic of the single stage SWGSR model

\subsubsection{Physical Properties}

The syngas heat capacity is calculated assuming ideal mixture, as shown in Eqn. (6.1).

$$
C_{p}=\sum_{i=1}^{N} y_{i} C_{p, i}
$$

The viscosity of the syngas, $\mu$, is estimated from Eqn. (6.2) (Adams, 2009) as:

$$
\mu=\sum_{i=1}^{N} \frac{y_{i} \mu_{i}}{\sum_{j=1}^{N} \sqrt{M_{j} / M_{i}}}
$$

where $M$ is molecular weight of species denoted by indices $i$ and $j$.

Assuming interactions between all pairs in the syngas, thermal conductivity of the mixture can be approximated by using the molar average thermal conductivity, Eqn. (6.3).

$$
\lambda=\sum_{i=1}^{N} y_{i} \lambda_{i}
$$


The effective diffusivity, $D_{e f f}$, is related to binary diffusivity, $D_{i j}$ (Adams, 2009), through Eqn. 6.4(6.4).

$$
D_{e f f, i j}=D_{i j}\left(\frac{\varepsilon}{\tau}\right)
$$

It is difficult to find accurate tortuosity values $(\tau)$ for the catalyst; however, since the tortuosity of water gas shift catalysts are in the range of 2-9, a tortuosity value of 5 is chosen (Adams, 2009). The porosity of the catalyst, $\varepsilon$, is assumed to be 0.38 (Francesconi et al., 2007). The binary diffusivity, $D_{i j}$, is the binary diffusivity of species $i$ into species $j$. An approximate equation for diffusion of species $i$ into a mixture is given as (Adams et al., 2009):

$$
D_{i, m}=\frac{1-y_{i}}{\sum_{j \neq i}\left(\frac{y_{j}}{D_{e f f, i j}}\right)}
$$

An analysis of the diffusivity of reactants, $\mathrm{CO}$ and $\mathrm{H}_{2} \mathrm{O}$, into the mixture in an industrial scale reactor showed that the diffusivity of $\mathrm{H}_{2} \mathrm{O}$ into the mixture is the lowest and thus considered as the rate limiting for the WGS reaction. The binary diffusivities are calculated using Eqns. (6.6a)(6.6b) (Adams et al., 2009; Satterfield et al., 1981).

$$
\begin{gathered}
D_{i j}=\left(A T^{B} / P\right)[\ln (C / T)]^{-2 D} \exp \left(-E / T-F / T^{2}\right) \\
D_{i j}=B / P
\end{gathered}
$$

Note that useful information for calculating the heat capacity, viscosity, thermal conductivity and binary diffusivity can be found in the work of Adams and Barton (2009). 


\subsubsection{Model Equation for Catalyst Pellets}

The 1-D heterogeneous model has been developed using the effectiveness factor to account for intra-particle mass transfer limitations. For a first-order reaction, the overall effectiveness factor relates the actual reaction rate, $r$, to the reaction rate evaluated at the bulk concentration using various system parameters, such as reaction rate constant, $k$, and mass transfer coefficient, $k_{c}$ (Hakkarainen et al., 1993). The equation for reaction rate is given in Eq. 6.7.

$$
-r_{A}=\Omega k C_{A, b u l k}
$$

where the overall effectiveness factor is defined as:

$$
\Omega=\frac{\eta}{1+\eta k / k_{c} a_{c}}
$$

The effectiveness factor is a function of Thiele modulus, $\phi_{t}$, and for a spherical catalyst it is calculated using Eq. 6.9.

$$
\eta=\frac{3}{{\phi_{t}}^{2}}\left(\phi_{t} \operatorname{coth} \phi_{t}-1\right)
$$

Eq. 6.10 is used to calculate the Thiele modulus, $\phi_{t}$.

$$
\phi_{t}=\frac{d_{c a t}}{2} \sqrt{\frac{k}{D_{e f f}}}
$$

The mass transfer coefficient can be calculated from Thoenes-Kramers correlation (Thoenes Jr et al., 1958) as shown in Eq. 6.11.

$$
k_{c}=\frac{1-\varepsilon}{\varepsilon} \frac{D_{i, m}}{d_{c a t}} R e^{1 / 2} S c^{1 / 3}
$$

where the diffusion of $\mathrm{H}_{2} \mathrm{O}$ into the mixture is considered for $D_{i, m}$ since it is rate limiting. Schmidt number, $S c$, and Reynolds number, $R e$, are calculated using Eqs. 6.12 and 6.13

$$
\begin{gathered}
S c=\frac{\mu}{\rho D_{e}} \\
R e=\frac{\rho u d_{c a t}}{\mu(1-\varepsilon)}
\end{gathered}
$$


The surface area per unit volume of the pellet, $a_{c}$, is estimated by Eqn. 6.14 and assuming ideal gas behavior, the linear gas velocity, $u$, is given by Eqn. 6.15 (Adam et al., 2009; Francesco et al., 2007).

$$
\begin{gathered}
a_{c}=6(1-\varepsilon) / d_{\text {cat }} \\
u=\frac{G R T}{P}
\end{gathered}
$$

Here, $G$ is the molar flux, $R$ is the universal gas constant and $T$ and $P$ are the temperature and pressure. 


\subsubsection{Species Balance}

Conservation equations are derived for all gas phase species is shown in Eq. 6.16.

$$
\frac{\partial C_{i}}{\partial t}=-\frac{1}{A_{c} \varepsilon} \frac{\partial F_{i}}{\partial z}+\left(\sum r_{i}\right) \frac{1-\varepsilon}{\varepsilon}
$$

Eq. 6.16 can be rewritten assuming ideal gas behavior for the syngas mixture (Adams et al., 2009) to give Eq. 6.17.

$$
\frac{\partial C_{i}}{\partial t}=-G R \frac{T_{g a s}}{P} \frac{\partial C_{i}}{\partial z}-C_{i} G R\left[\frac{1}{P} \frac{\partial T_{g a s}}{\partial z}-\frac{T_{g a s}}{P^{2}} \frac{\partial P}{\partial z}\right]+\left(r_{W G S, i}+r_{H y d, i}\right) \frac{1-\varepsilon}{\varepsilon}
$$

where $C_{i}$ is the molar concentration of species $i, z$ is the axial position. The molar flux, $G$, is calculated using Eqn. 6.18. $F_{0}$ is the total inlet molar flow rate, entering the reactor with diameter $d_{\text {rct }}$ (Adams et al., 2009).

$$
G=\frac{4 F_{0}}{\pi d_{r c t}^{2} \varepsilon}
$$

The boundary condition at the inlet to the reactor $(a t z=0)$ can be expressed as $C_{i}=C_{i, i n}$, $T_{\text {gas }}=T_{\text {in }}$, and $P=P_{\text {in }}$, where $C_{i, i n}, T_{i n}$ and $P_{\text {in }}$ are the concentration, temperature and pressure of the gas at the inlet to the reactor.

\subsubsection{Momentum Balance}

A simplified momentum conservation equation is considered assuming pseudo-steady state. This is done by calculating the pressure drop along the reactor. The Ergun equation is used for calculating the axial pressure profile in a packed bed, rewritten as Eq.6.19 (Ergun, 1952).

$$
\frac{d P}{d z}=\frac{\rho u^{2}}{d_{c a t}}\left(\frac{1-\varepsilon}{\varepsilon^{3}}\right)\left(1.75+\frac{150}{R e}\right)
$$

where $\rho$ is the density of the fluid. 


\subsubsection{Gas Phase Energy Balance}

The temperature variation across the reactor can be obtained by deriving the gas phase energy balance:

$$
\frac{\partial T_{g a s}}{\partial t}=\frac{1}{\rho_{g a s} C_{p}}\left[-C_{p} G \frac{\partial T_{g}}{\partial z}+\frac{h_{f} a_{c}}{\varepsilon}\left(T_{c a t}-T_{g a s}\right)\right]
$$

where the heat transfer coefficient, $h_{f}$, can be estimated using Eq. 6.21 (Satterfield et al., 1981).

$$
h_{f}=1.37\left(\frac{0.357}{\varepsilon}\right)\left(C_{p} G M\right)\left(\frac{\mu}{d_{c a t} G M}\right)^{0.359}\left(\frac{\lambda M}{C_{p} \mu}\right)^{2 / 3}
$$

The boundary condition can be expressed as $T_{\text {gas }}($ at $z=0)=T_{\text {in }}$, where $T_{\text {in }}$ is the temperature of the gas at the inlet to the reactor.

\subsubsection{Catalyst Phase Energy Balance}

Assuming that the temperature only varies in the $z$ direction and neglecting radial temperature profile, the adiabatic energy balance for the catalyst phase is show in Eq. 6.22.

$$
\begin{gathered}
\frac{\partial T_{c a t}}{\partial t}=\frac{1}{\rho_{c a t} C_{p, c a t}}\left[K_{c a t} \frac{\partial^{2} T_{c a t}}{\partial z^{2}}-\frac{h_{f} a_{c}}{1-\varepsilon}\left(T_{c a t}-T_{g a s}\right)+r_{W G S} \Delta H_{R, W G S}\right. \\
\left.+r_{H y d} \Delta H_{R, h y d}\right]
\end{gathered}
$$

For the catalyst phase temperature, the boundary conditions considered are: $\partial T_{\text {cat }} / \partial z(z=L)=$ 0 and $T_{\text {cat }}(z=0)=T_{\text {gas }}$. Thermal conductivity of the catalyst is assumed to be the same as pure alumina, $35 \mathrm{~W} / \mathrm{m}-\mathrm{K}$. Additionally, this equation requires the calculation of the heats of reaction using the enthalpy using Eq. 6.23.

$$
H_{i}=\Delta H_{298}^{f}+\int_{298}^{T} C_{p, i}(T) d T
$$

The standard heat of formation of $\mathrm{CO}, \mathrm{CO}_{2}, \mathrm{COS}, \mathrm{H}_{2} \mathrm{O}$ and $\mathrm{H}_{2} \mathrm{~S}$ are -110.5, -393.5, -142, -241.9, and $-20.63 \mathrm{~kJ} / \mathrm{mol}$, respectively and are obtained using Aspen Properties model (AspenTech, 2009). 


\subsubsection{Reaction Kinetics}

Although the WGS reaction has been studied over sulfur-tolerant catalysts, such as Co/Mo catalyst, kinetics of the COS hydrolysis on the sour shift catalyst are rarely reported. Thus, the parameters of COS hydrolysis are derived from the open literature for alumina-based catalyst (Svoronos et al., 2002). However, the parameters for the WGS reaction need to be obtained by analyzing the available experimental data (Berispek et al., 1975). It was found that measurement errors were present in the data collected in experimental studies; a data reconciliation procedure is performed to obtain consistent data (Mobed et al., 2014).

The rate equation for COS hydrolysis considering Eley-Rideal mechanism (Svoronos et al., 2002) is shown in Eq. 6.24.

$$
-r_{h y d}=k_{H y d} \frac{P_{C O S}}{1+K_{e q, h y d} P_{H_{2} O}}
$$

where the partial pressures are in $\mathrm{kPa}$. The rate and equilibrium constants are using Eq. 6.25 (Svoronos, 2002).

$$
\begin{aligned}
& k_{h y d}=4223.32 \exp \left(\frac{-25270\left[\frac{\mathrm{J}}{\mathrm{mol}}\right]}{R T_{\text {cat }}}\right) \\
& K_{\text {eq,hyd }}=\exp \left(\frac{10010[\mathrm{~K}]}{T_{\text {cat }}}-15.89\right)
\end{aligned}
$$

Considering a pseudo-first order equilibrium reaction, the rate equation for the WGS reaction can be written as Eq. 6.27.

$$
-r_{W G S}=\Omega k_{W G S} P\left(x_{C O}-\frac{x_{\mathrm{CO}_{2}} x_{\mathrm{H}_{2}}}{K_{e q, W G S} x_{\mathrm{H}_{2} \mathrm{O}}}\right)
$$

where $P$ is the pressure. The rate constant, $k_{W G S}$, follows the Arrhenius equation and the equilibrium constant, $K_{e q, W G S}$, are calculated as given by Moe et al. (1962) using Eq. 6.28 and 6.29 .

$$
\begin{gathered}
k_{W G S}=k_{0} \exp \left(\frac{-E_{a}}{R T}\right) \\
K_{e q, W G S}=\exp \left(\frac{4577.8[K]}{T_{c a t}}-4.33\right)
\end{gathered}
$$




\subsubsection{Pressure Scale-up}

The reaction kinetics derived from experimental data obtained at lower pressure are not applicable to industrial-sized reactors since they result in over-prediction of the reaction rates by orders of magnitude. Therefore, a pressure scale-up factor is used to address such overpredictions at high pressures. The reaction rate at higher pressures is related to the rate at atmospheric pressure (Singh et al., 1977) using Eq. 6.30.

$$
r_{W G S}^{\prime}=P_{\text {scale }} r_{W G S}
$$

Here $P_{\text {scale }}$ is the pressure scale factor which is calculated using Eq. 6.31 (Adams et al., 2009).

$$
P_{\text {scale }}=P^{\left(0.5-\frac{P}{500}\right)}
$$

The pressure, $P$ is in atm. Eqn. 6.31 implies that the reaction rate above atmospheric pressure is in the range of 1-5 times the reaction at atmospheric pressure and the equation is reported to be valid up to $55 \mathrm{~atm}$ (Adams et al., 2009). Therefore, the rate equation for the WGS reaction in Eqn. 6.27 is rewritten for high pressures as Eq. 6.32.

$$
-r_{W G S}^{\prime}=\Omega k_{W G S} P_{\text {Scale }}\left(x_{C O}-\frac{x_{\mathrm{CO}_{2}} x_{\mathrm{H}_{2}}}{K_{e q, W G S} x_{\mathrm{H}_{2} \mathrm{O}}}\right)
$$

\subsubsection{Catalyst Deactivation}

The catalyst loses its activity over time mainly due to poisoning, fouling, and thermal and mechanical degradation. However, for the current studies in a one-stage water gas shift reactor, the catalyst is assumed to deactivate only due to thermal degradation (sintering). The catalyst activity is defined in terms of reaction rates for both WGS reaction and COS hydrolysis (Giunta et al., 2006) as:

$$
a_{(t)}=\frac{r_{(t)}}{r_{(0)}}
$$

The catalyst deactivation equation given by Giunta et al. (2006) is used in the present work and is shown in Eq. 6.34. 


$$
\frac{d a}{d t}=k_{d}\left(a-a_{\infty}\right)^{m}
$$

where $m$ is the order of sintering and is reported to be either 1 or $2 ; a_{\infty}$ is limiting activity at infinite time; and $k_{d}$ is the sintering rate constant. In the work of Giunta et al. (2006), the catalyst activity is given at some point in time which gives a good estimate for the $a_{\infty}$ in this work. Also here, $m$ is assumed to be 2 and $k_{d}$ is found by integrating Eqn. 6.34 for expected life time of Co/Mo catalyst, 5 years, until the catalyst reaches $99 \%$ of its limiting activity (Rase et al., 1977; Bartholomew et al., 2001; Giunta et al., 2006).

\subsection{Solution Approach}

The system consists of a set of partial differential equations (PDEs) representing the state of the system. The PDEs in the modeling equations are converted to ODEs using the method of lines, where the spatial derivatives are discretized using a backwards difference method. However for solving the equations, the rate parameters for the water gas shift reaction need to be obtained from the available experimental data (Berispek et al., 1975). The experimental data were generated from an isothermal reactor under steady-state conditions. Therefore, the energy balance equations are eliminated to achieve an isothermal reactor and the time derivatives are set to zero. The resulting set of nonlinear equations is solved using a trust-region-dogleg algorithm by 'fsolve' function in MATLAB. Later, a dynamic adiabatic reactor is simulated by scaling up the reactor and using the obtained parameters. Equations are solved for the 26 meter long industrial reactor considering 300 grid points.

A typical industrial sour gas shift process operates adiabatically and typically under high pressure. Only the first stage of the WGS reactor system was modeled initially to observe the profiles of key variables due to the reconciled kinetics. The one stage model is used to perform sensor placement for a condition monitoring based on catalyst deactivation. The two-stage SWGSR system model is combined with the SELEXOL unit model to simulate faults in the gasification island. These results will be discussed in Chapter 7. 


\subsection{Results - Single Stage SWGSR}

The syngas in this simulation is composed of $\mathrm{H}_{2}, \mathrm{CO}, \mathrm{CO}_{2}, \mathrm{H}_{2} \mathrm{~S}, \mathrm{H}_{2} \mathrm{O}$ and $\mathrm{COS}$ with mole fractions of $0.21929,0.23021,0.08880,0.00465,0.45696$ and $9 \times 10^{-5}$, respectively (Woods, 2007). The reactor is filled with "Aldridge" (Aldridge, 1974) catalyst, catalyst Q, $2.2 \mathrm{~mm}$ in diameter and with porosity of 0.38 . It is assumed that no heat loss takes place from the reactor to the surrounding (Berispek et al., 1975). With given composition and pressure for an IGCC case study, the reactor volume and the inlet temperature are adjusted to size a reactor with $10 \%$ overdesign and assuming length to diameter $(L / D)$ of about 5.5 (Woods et al.,2007). Table 6.1 shows the sizing and operating conditions of the reactor.

Table 6.1: Simulation condition (Woods et al., 2007)

\begin{tabular}{cc}
\hline Condition & Value \\
\hline Length (m) & 29 \\
Diameter (m) & 5.2 \\
Flow (kmol/s) & 4.9 \\
Inlet Temperature (K) & 620 \\
Inlet Pressure (atm) & 54.43 \\
\hline
\end{tabular}




\subsubsection{Steady State Results}

\subsubsection{CO and COS Profiles}

Figures 6.2-6.3 show the conversion of $\mathrm{CO}$ and $\mathrm{COS}$ along the reactor for different inlet gas temperatures. As seen in Figure 6.2, the conversion at the outlet remains fairly constant. However, the conversion along the reactor reduces as the inlet temperature decreases. Although the conversion of $\mathrm{CO}$ is affected by the change in the inlet temperature albeit slightly, the COS conversion is not affected at higher inlet temperatures as seen in Figure 6.3. The inlayed block in Figure 6.2 shows that the final $\mathrm{CO}$ conversion decreases as the inlet temperature changes from $620 \mathrm{~K}$. Although the final values of $\mathrm{CO}$ conversion at the end of the reactor for the lower temperature case are lower than the base case, their slopes appear to indicate continuing reaction. The higher temperature cases appear to be more leveled out, indicating they are approaching equilibrium at the end of the reactor.

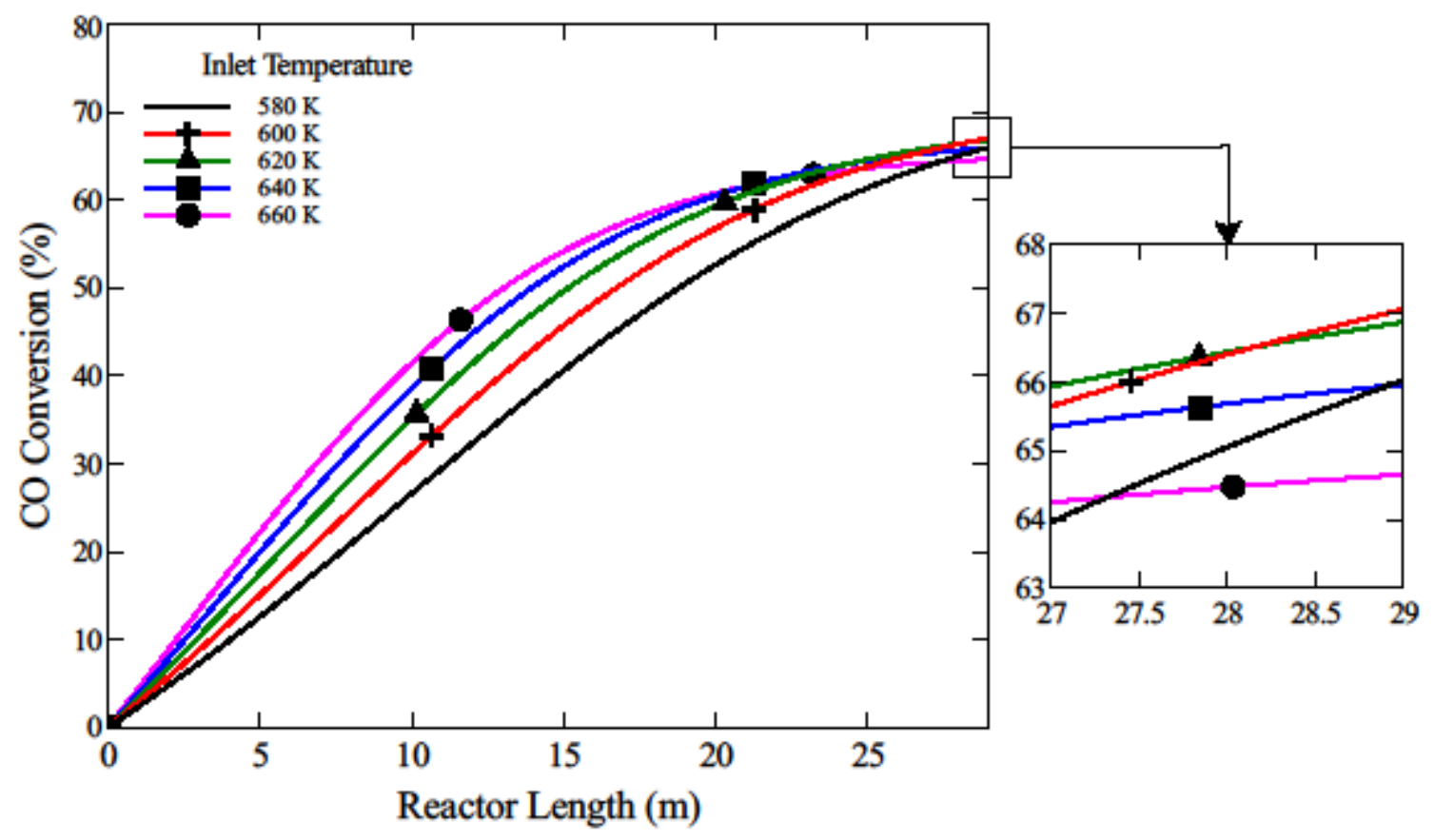

Figure 6.2: $\mathrm{CO}$ conversion profiles for different inlet gas temperatures 


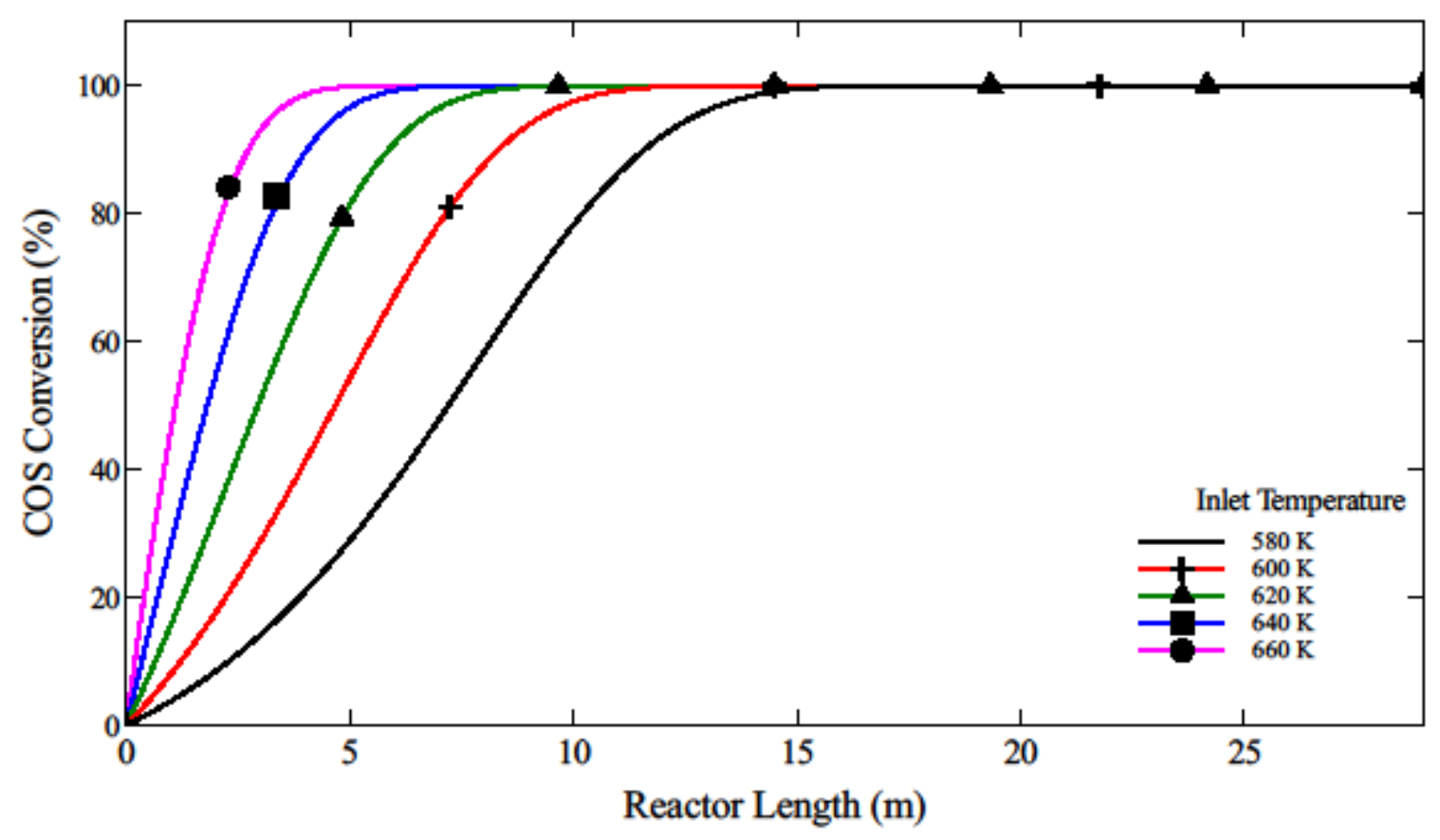

Figure 6.3: COS conversion profiles for different inlet gas temperatures

Figure 6.3 shows that high conversion is achieved at temperatures around the inlet operating temperature of $620 \mathrm{~K}$ and all the inlet temperature cases studied appear to bring the COS hydrolysis reaction to completion. 


\subsubsection{2 $\mathrm{H}_{2} \mathrm{O} / \mathrm{CO}$ Ratio}

In IGCC plants, additional steam is added to the syngas feed to achieve the desired conversion of CO. However, the required steam is extracted from the steam turbine (Bhattacharyya, 2010) which results in a decrease in the production of electricity from the steam turbines. It is therefore important to design the $\mathrm{H}_{2} \mathrm{O} / \mathrm{CO}$ ratio at the inlet of the WGS reactors appropriately by considering an optimal CO conversion in the WGS reactor system. Figure 6.4 show the relation between $\mathrm{CO}$ conversion and $\mathrm{H}_{2} \mathrm{O} / \mathrm{CO}$ ratio. In Figure 6.4, increasing the molar ratio of steam to $\mathrm{CO}$ at constant dry flow rate $(2,661 \mathrm{~mol} / \mathrm{s})$ increases the $\mathrm{CO}$ conversion until it reaches a maximum at a steam-to- $\mathrm{CO}$ ratio of about 4 . However, increasing the ratio requires higher flow rate of steam, consequently, higher flow rate at the inlet to the reactor. Increasing the flow at the same residence time and superficial velocity requires higher reactor volume. This can be seen in Figure $6.5 \mathrm{a}$ and Figure $6.5 \mathrm{~b}$ where the reactor diameter and the flow are non-dimensionalized with respect to the values in Table 6.1. Thus, higher conversion must be weighed with respect to the capital cost of the reactor and the amount of steam taken from the steam cycle. The increase in steam could reduce the reaction rate of the COS hydrolysis reaction, as seen in Eq. 6.24. However, it is found that the COS conversion is not greatly affected since it reached completion very early in the reactor.

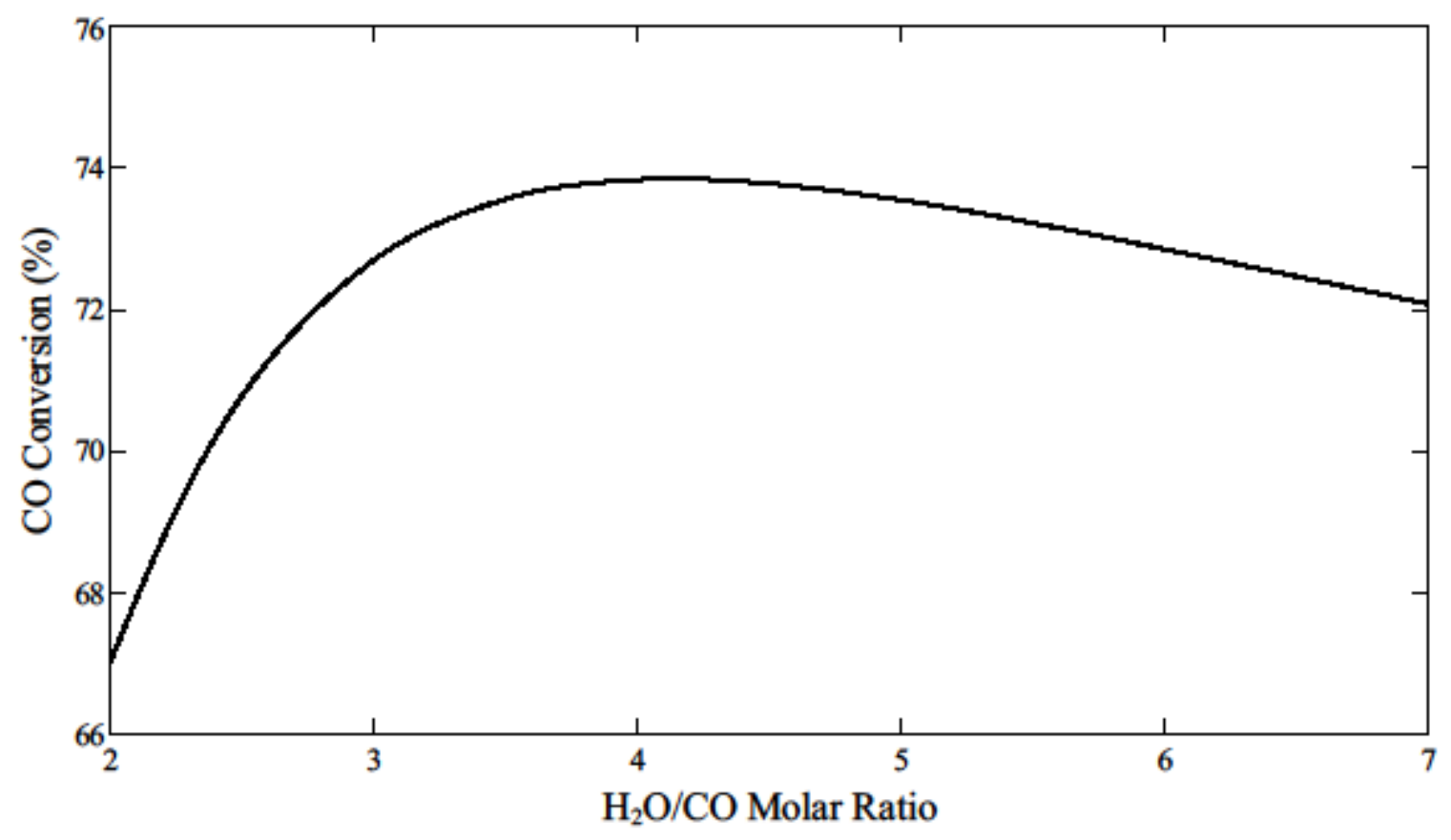

Figure 6.4: $\mathrm{CO}$ conversion profile for different steam/CO molar ratio 

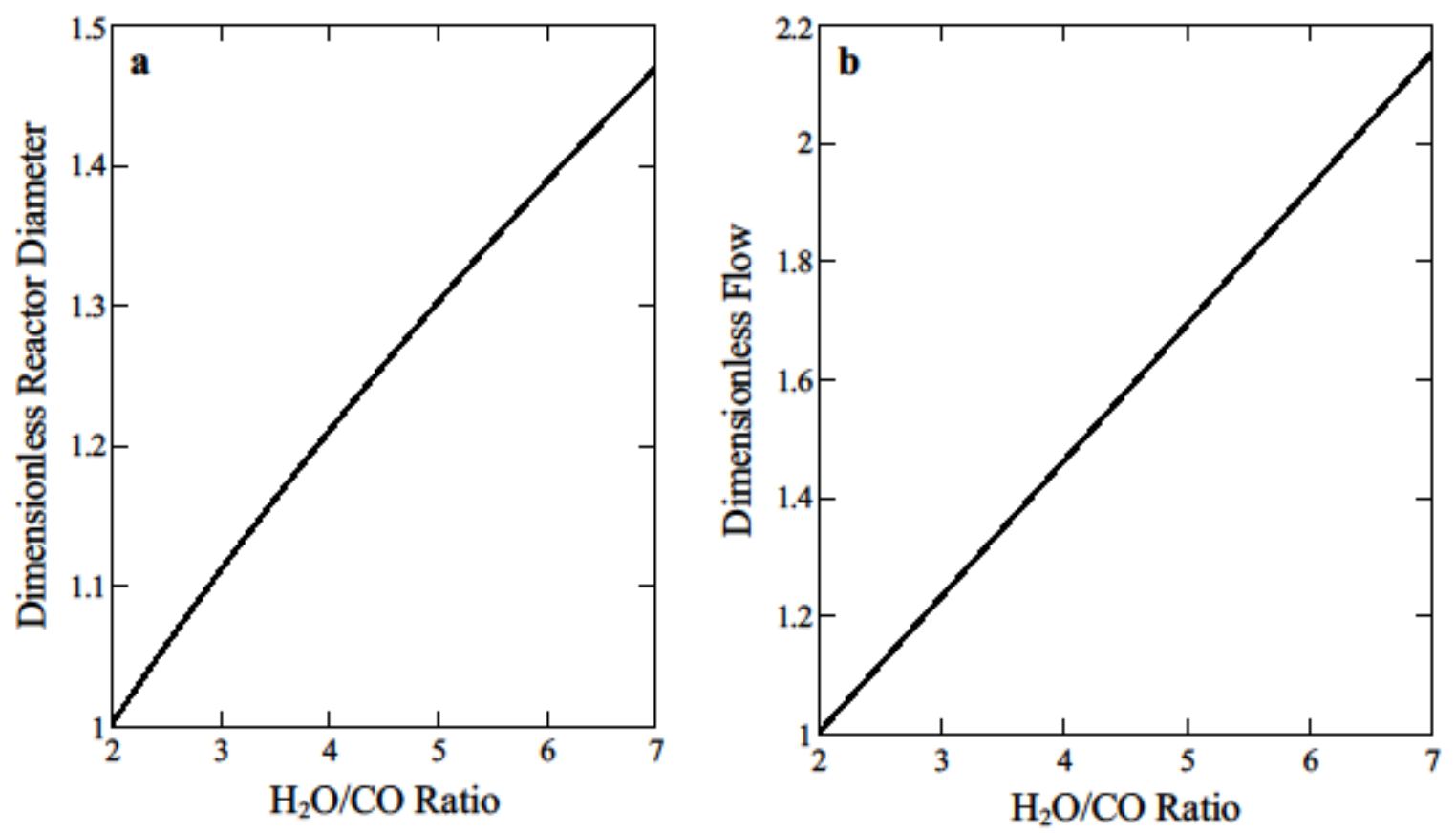

Figure 6.5: Required (a) Diameter (b) Flow at different steam/CO molar ratios 


\subsubsection{Dynamic Results}

\subsubsection{Change in Inlet Temperature}

When a step increase in the inlet temperature from 620 to $640 \mathrm{~K}$ is simulated, the reactor initially shows an inverse response in the CO conversion as seen in Figure 6.6. As the inlet temperature rises, it takes some time for the temperature in the rest of the reactor to increase. Initially the $\mathrm{CO}$ conversion increases due to higher reaction rate, but decreases later as the reaction temperature rises pushing the equilibrium to the left.

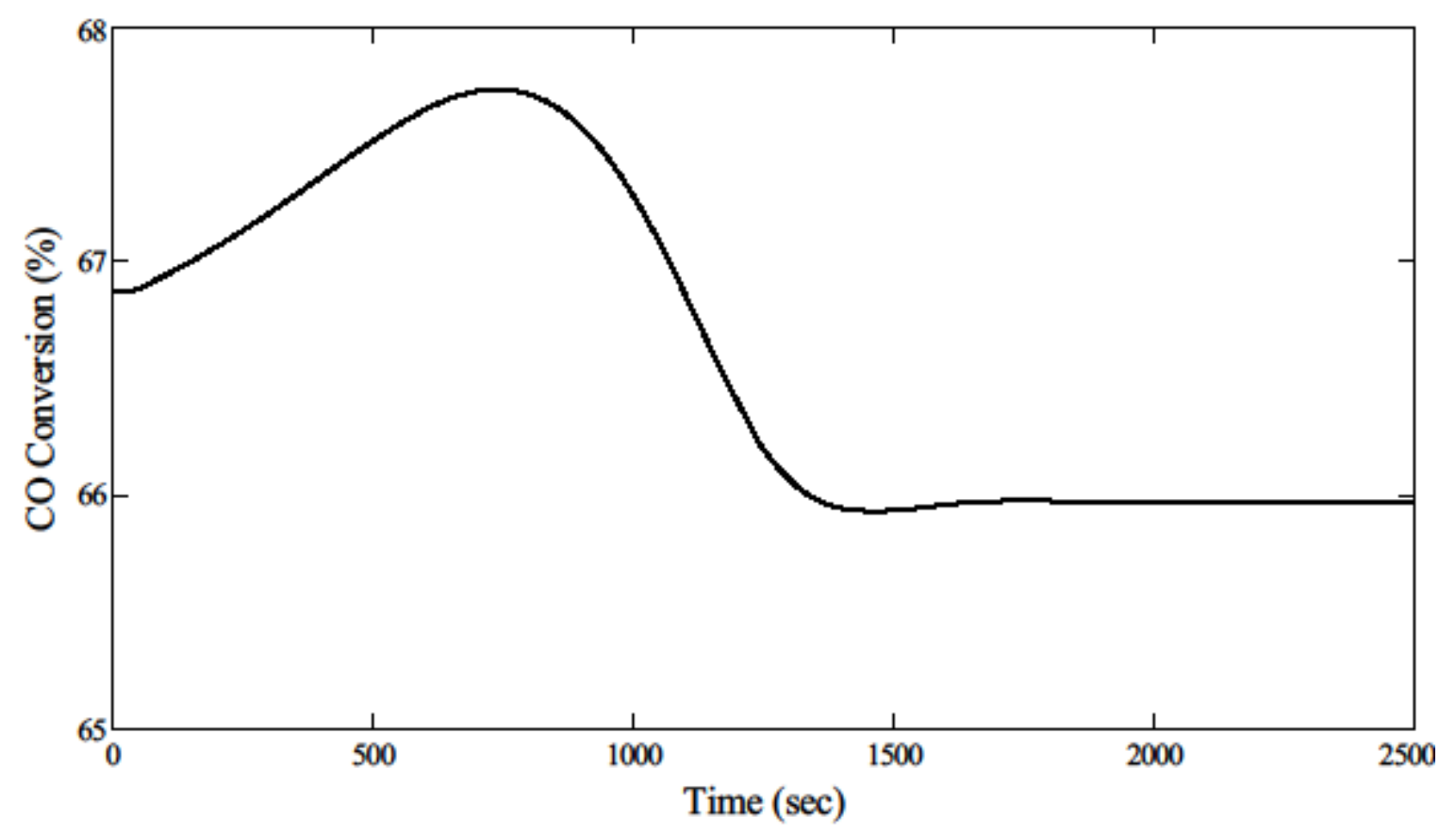

Figure 6.6: $\mathrm{CO}$ conversion transient for a step change in inlet temperature from 620 to $640 \mathrm{~K}$

The dynamic response of gas temperature at the outlet of the reactor for the same step increase in the inlet temperature is seen in Figure 6.7. When the inlet temperature increases, more $\mathrm{CO}$ is consumed in the area near the inlet of the reactor, thus, the CO conversion increases initially as seen in Figure 6.6. This causes the $\mathrm{CO}$ concentration to decrease in the rest of the reactor, which at the same time, reduces the reaction rate. Since reaction rate is decreased, less heat is generated by the exothermic reactions, thus, the temperature decreases initially. However, as the catalyst temperature slowly increases due to the higher heat input from the front end, the temperature increases. The COS conversion remains unchanged as the reactor temperature remains high enough to bring the reaction to completion in the early region of the reactor. 


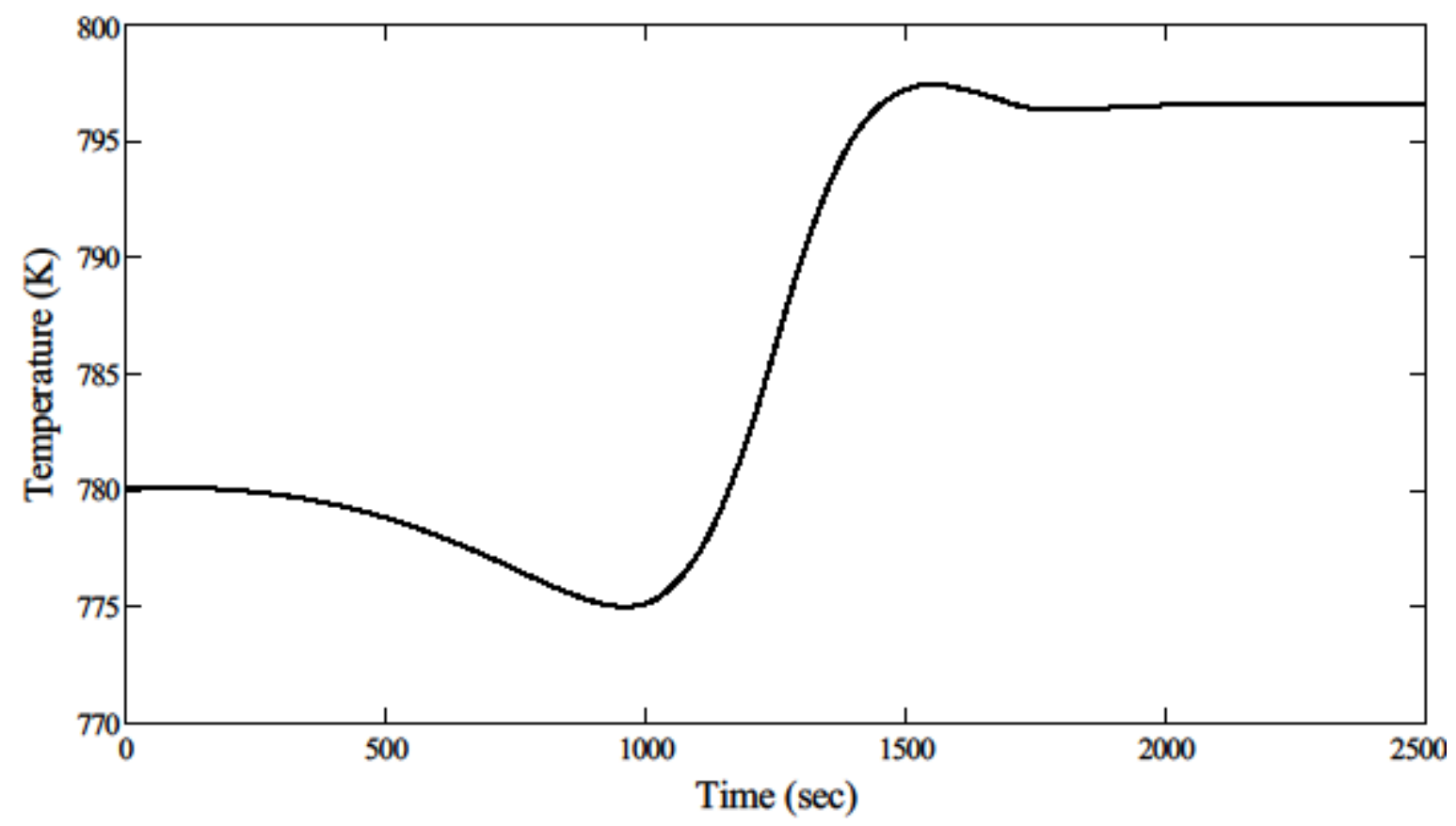

Figure 6.7: Temperature transient at the outlet of the reactor for a step change in inlet temperature from 620 to $640 \mathrm{~K}$

\subsubsection{Fault Simulation - Catalyst Deactivation}

The catalyst deactivation due to sintering can lower the conversion in a sour WGS reactor. Figure 6.8 shows the effect of catalyst deactivation on $\mathrm{CO}$ conversion for different inlet temperatures over the catalyst lifetime of 60 months (Rase et al., 1977). Figure 6.8 shows that at lower inlet temperatures, catalyst deactivation has substantial effect on the conversion of $\mathrm{CO}$ during the lifetime of the catalyst. Therefore, although a lower inlet temperature would be preferred from the perspective of cost to heat the syngas, the conversion of $\mathrm{CO}$ reduces drastically over time at lower temperatures.

The COS reaction appears to reach completion for the range of inlet temperature cases considered as can be seen in Figure 6.9. This, of course, depends upon the length of the reactor. The results, once again show a drastic decrease in conversion for the same length of reactor for a lower inlet temperature. 


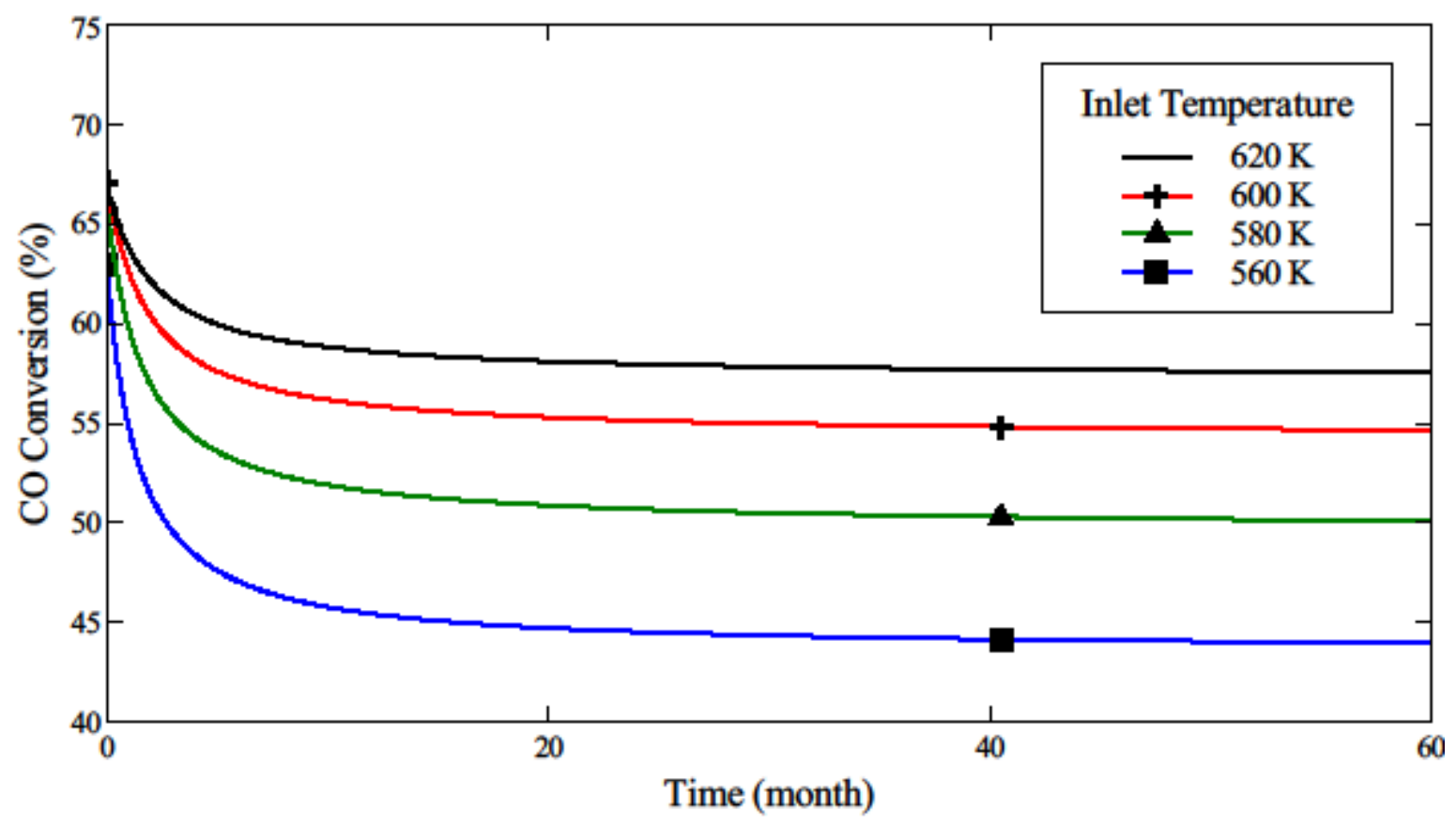

Figure 6.8: Effect of catalyst deactivation over time on $\mathrm{CO}$ conversion over 5 years

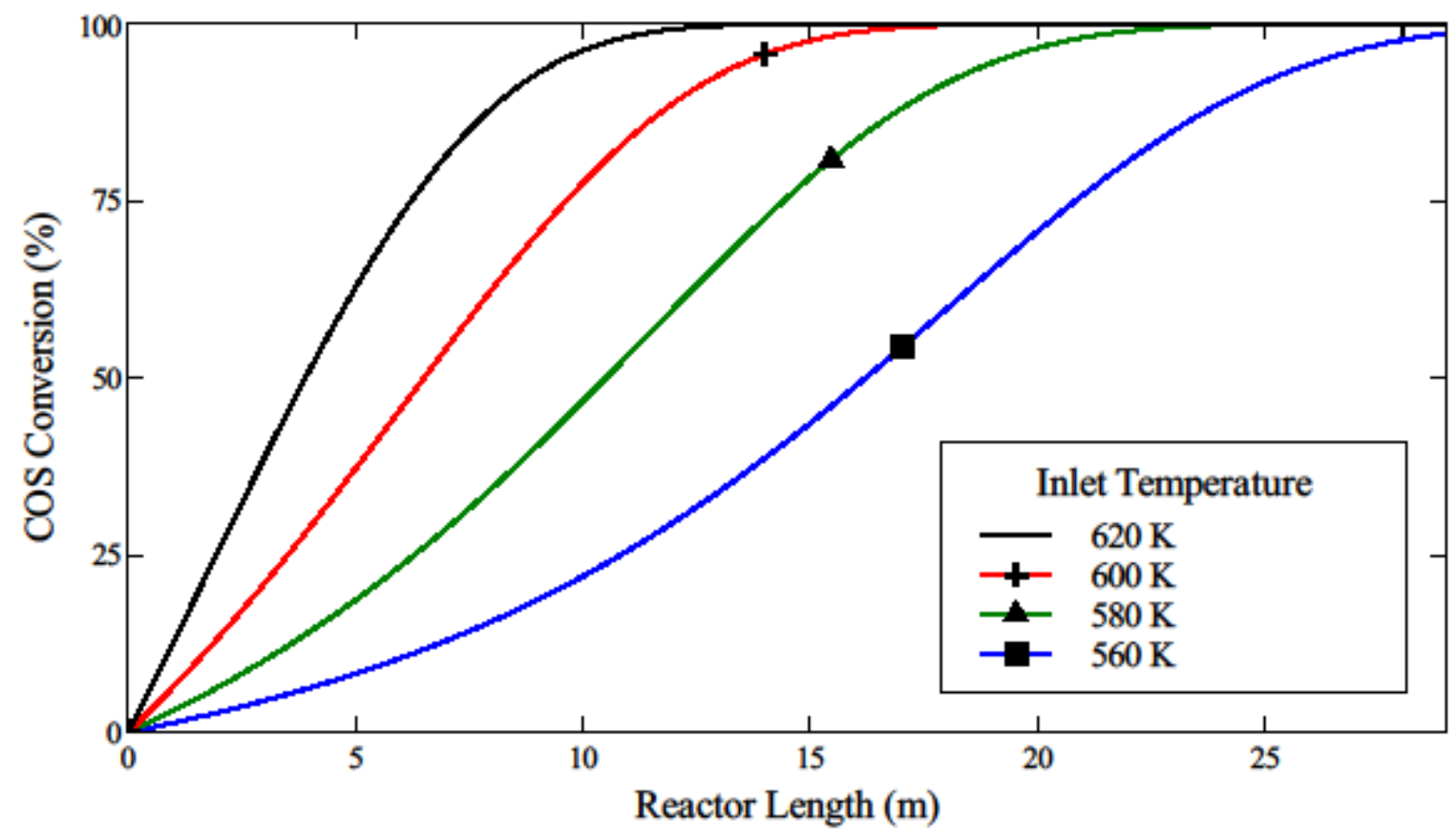

Figure 6.9: Effect of catalyst deactivation over time on COS conversion over 5 years 


\subsection{Model Enhancement to Two-stage SWGSR System}

Considering an overall target of $90 \%$ carbon capture in an IGCC plant, a two-stage WGS reactor system with high- and low-temperature reactors arranged in series with inter-stage coolers is required (Bhattacharyya, 2010). The single stage SWGSR model developed here can easily be extended to this configuration. A second SWGSR model is developed similar to the first model that uses the outlet conditions of the first model as its inlet conditions. This reactor was sized to achieve equilibrium at about $90 \%$ of the length and the valve coefficients were set so as to match the flowrates. Both stages were run independently until they achieved steady state. Then, the code for the two stages was compiled into a one m-file in MATLAB and the SWGS reactor system was solved as a single unit. Equations for an inter stage heat exchanger (HE) is added to the model. In order to make the current model compatible with the flowrates expected in an IGCC plant, an additional train of two-stage SWGSR system is added. A single train of the reactor system is shown in Figure 6.10.

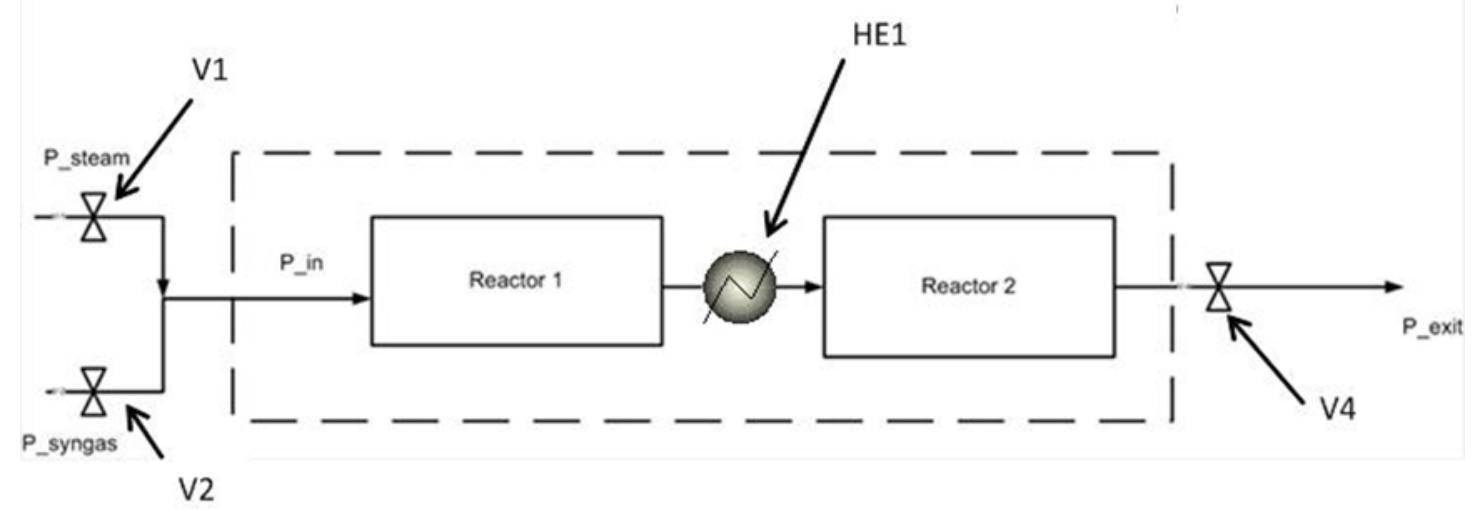

Figure 6.10: Schematic showing the two-stage SWGSR system

The system is pressure driven and the opening of the valves V1, V2 and V4 determine the flow through the system. Standard PID type control equations are added to the model to ensure the process behaves more like an industrial reactor. It is noted that in an industrial case, the valve v4 would not be located at the exit of the reactor system, and rather at the end of the AGR system. However, in order to close the system of equations and study the two-stage reactor system, this valve is added. 


\subsubsection{Fault Simulation - Two-Stage SWGSR}

Faults affecting the catalyst performance are simulated. The undesired accumulation of fly ash onto the catalyst of SWGSR could result in a decrease in the porosity of the catalyst or change its surface area. Undesired materials can also poison the catalyst resulting in reduction in the catalyst activity.

The following faults are simulated.

1. $10 \%$ reduction in the surface area of catalyst in the first reactor of the SWGSR within 22 minutes

2. $10 \%$ reduction in the porosity of catalyst in the first reactor of the SWGSR within 22 minutes

3. 5\% reduction in the surface area in each of the reactors of the SWGSR within 22 minutes

4. $5 \%$ reduction in the porosity of each of the reactors of the SWGSR within 22 minutes

5. $10 \%$ reduction in the catalyst activity in the first reactor of the SWGSR within 49 minutes.

6. 5\% reduction in the catalyst activity in each of the reactors of the SWGSR within 49 minutes.

These faults are simulated in the two-stage SWGSR system and the responses at the end of the second stage of the SWGSR are plotted and shown in Figures 6.11-6.13. The legend for the plots is shown in Table 6.2.

Table 6.2: Legend for Figures 6.3-6.5

\begin{tabular}{cc}
\hline Fault \# & Representation \\
\hline 1 & \\
2 & \\
3 & ----- \\
4 & ----- \\
5 & ---- \\
6 & - \\
\hline
\end{tabular}




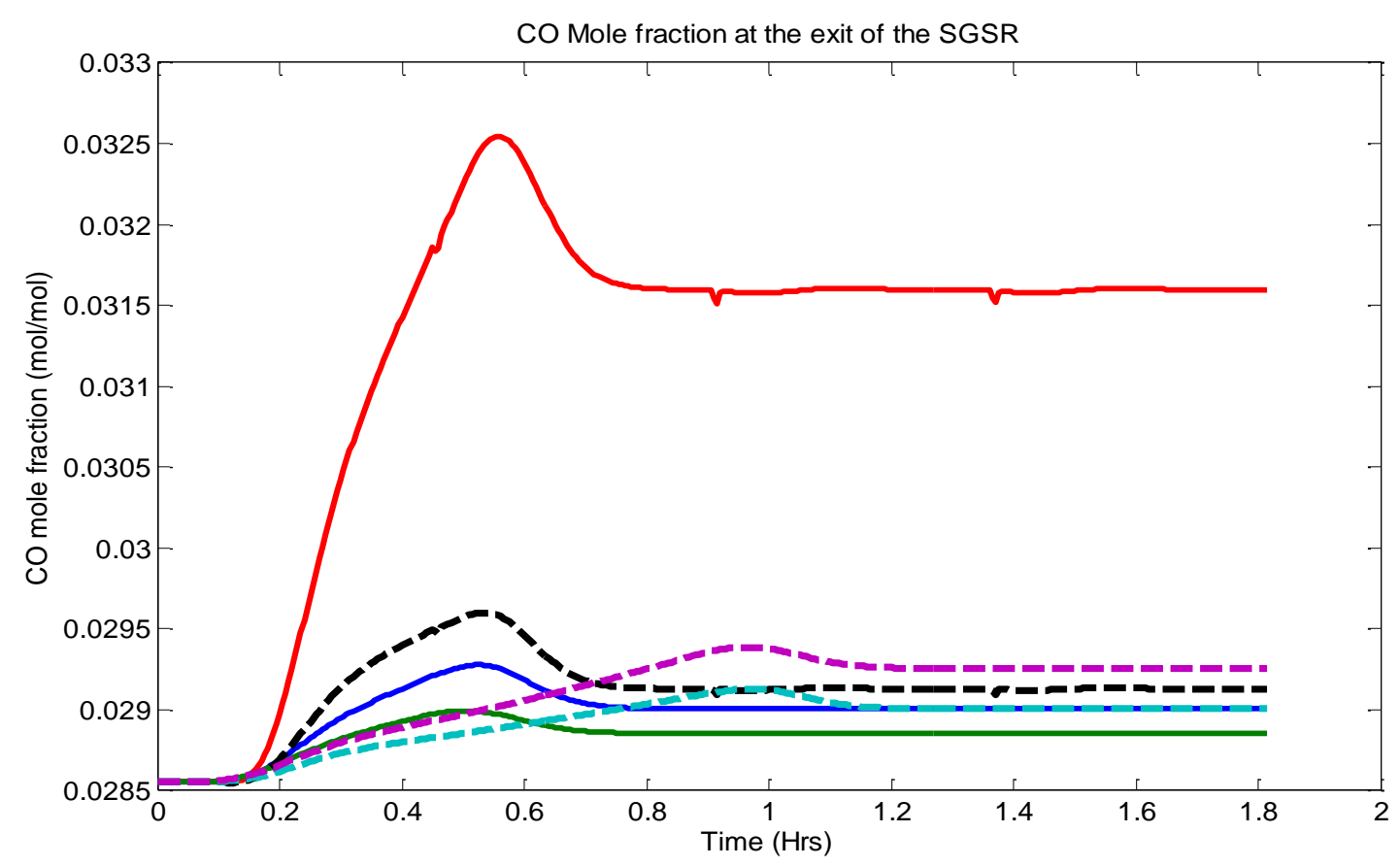

Figure 6.11: Change in CO mole fraction at the exit of the SWGSR due to faults

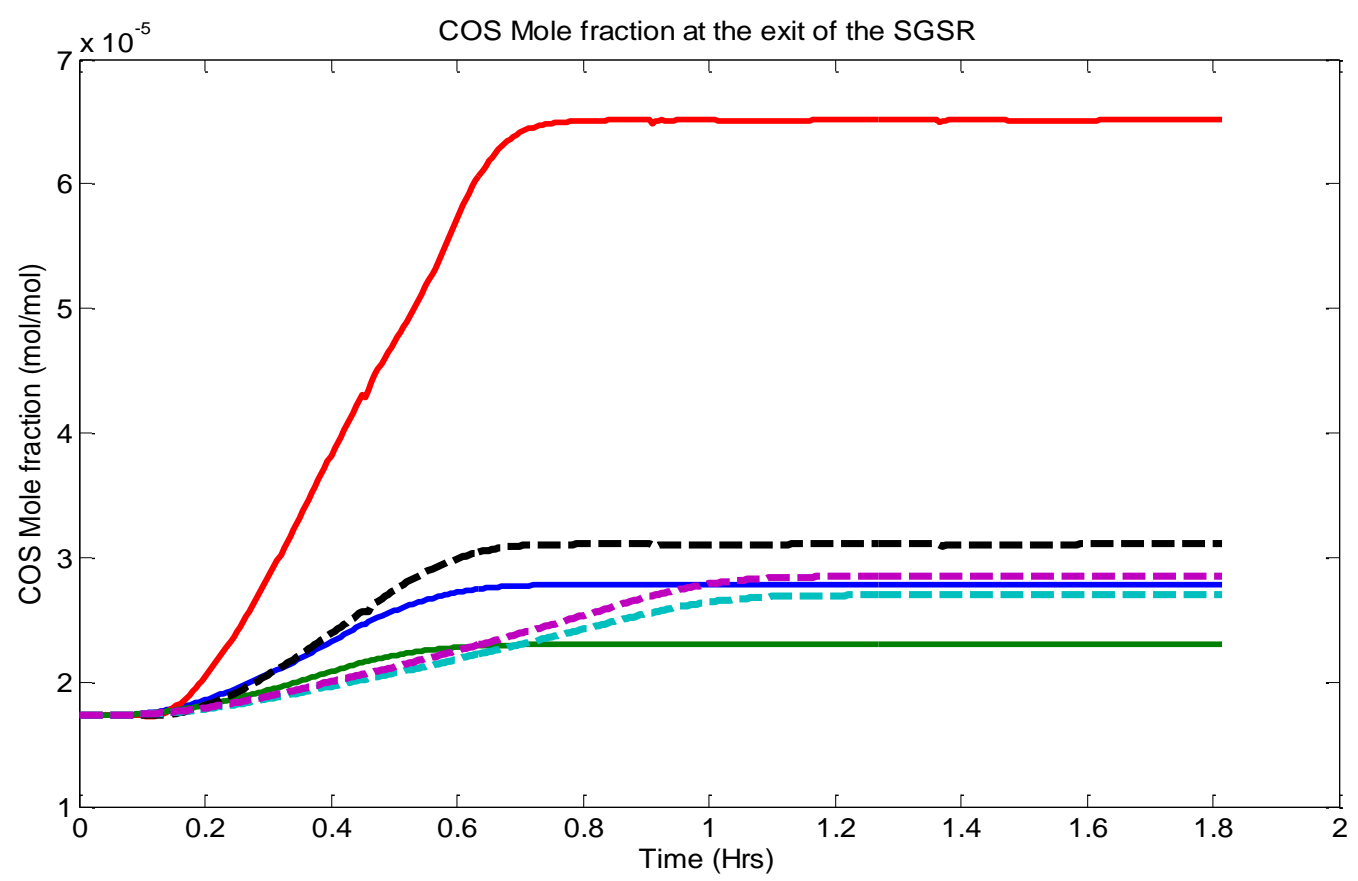

Figure 6.12: Change in COS mole fraction at the exit of the SWGSR due to faults 


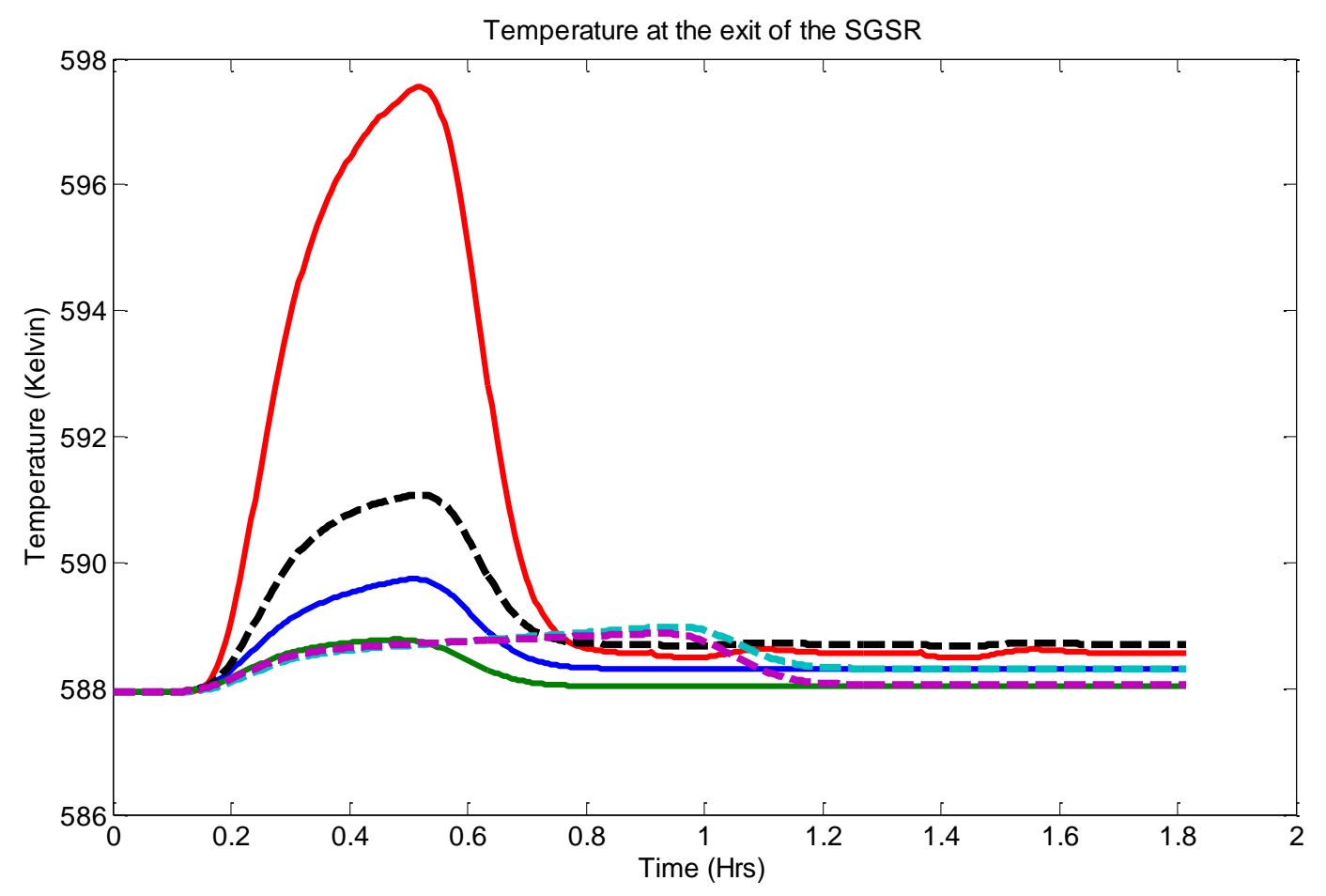

Figure 6.13: Change in outlet temperature of the SWGSR due to faults

The effect of the reduction in catalyst porosity in the first stage (R1) by $10 \%$ over 22 minutes appears to have the biggest response in the Figures 6.11-6.13. The extent of reaction of the WGS reaction and the COS hydrolysis reaction decreases considerably due to this fault. From Figure 6.13 , the temperature also appears to peak during the simulation of this fault. When the porosity stops changing, the temperature at the end of the SWGSR becomes stable, close to its initial value. A $5 \%$ change in porosity across both the reactors appears less detrimental to the $\mathrm{CO}$ and COS conversion in the reactor systems but is still higher than other faults.

Another important interpretation of these results can be obtained on the basis of selection of sensors to detect faults and the criteria to be used. For example, using a CO sensor, the scaled change in exit concentration due to the faults is smaller as compared with the COS sensor. However, although the faults result in at least a two fold increase in the COS mole fraction at the end of the reactor system, the concentration may be smaller than the resolution of the composition sensor.

A temperature sensor would be a cheaper option if only the initial response is used. However, based on the steady state response of the fault, this may be misleading. 
It should be noted that the variables that respond best will be selected by the sensor placement algorithm and the variables presented in the report are in order to show the extent and effect of the fault on a few key variables.

\subsubsection{Results - Two Stage SWGSR System}

After being cooled, the exit stream from the first stage (R1) is fed to the second stage (R2). The temperature profile of the syngas in the $\mathrm{R} 2$ is shown in Figure 6.14.

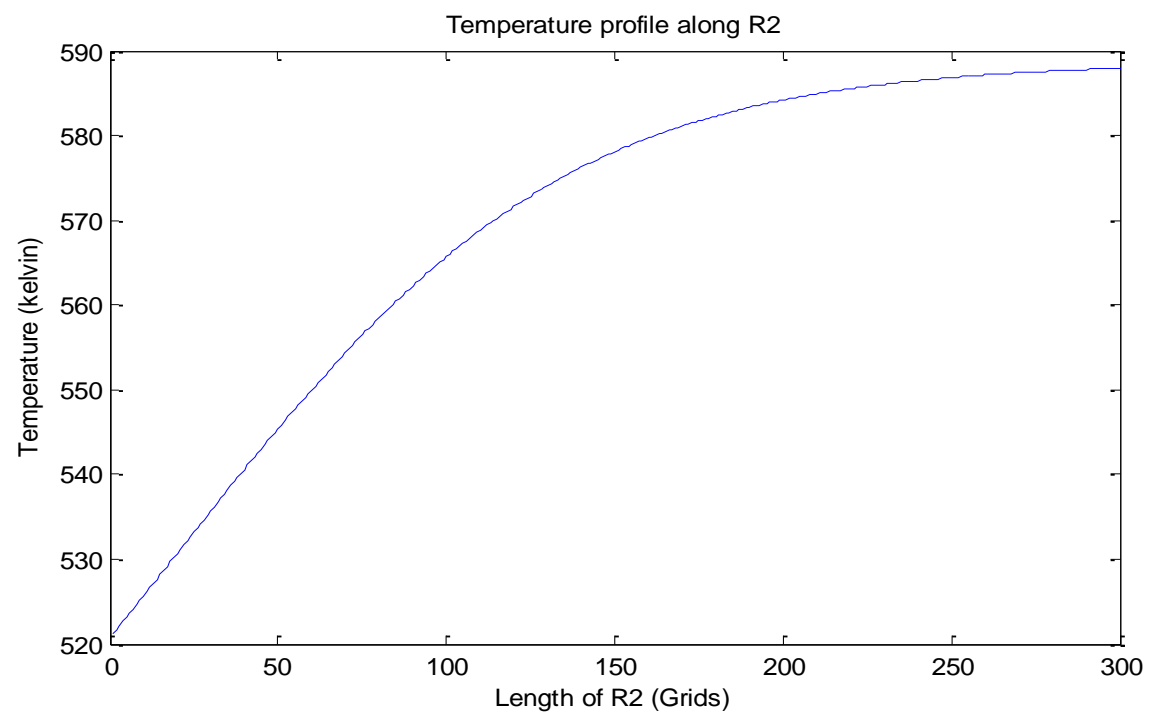

Figure 6.14: Temperature profile along reactor R2

Figure 6.14 shows that the temperature begins to level out at the end of R2 as the shift reaction approaches equilibrium. 


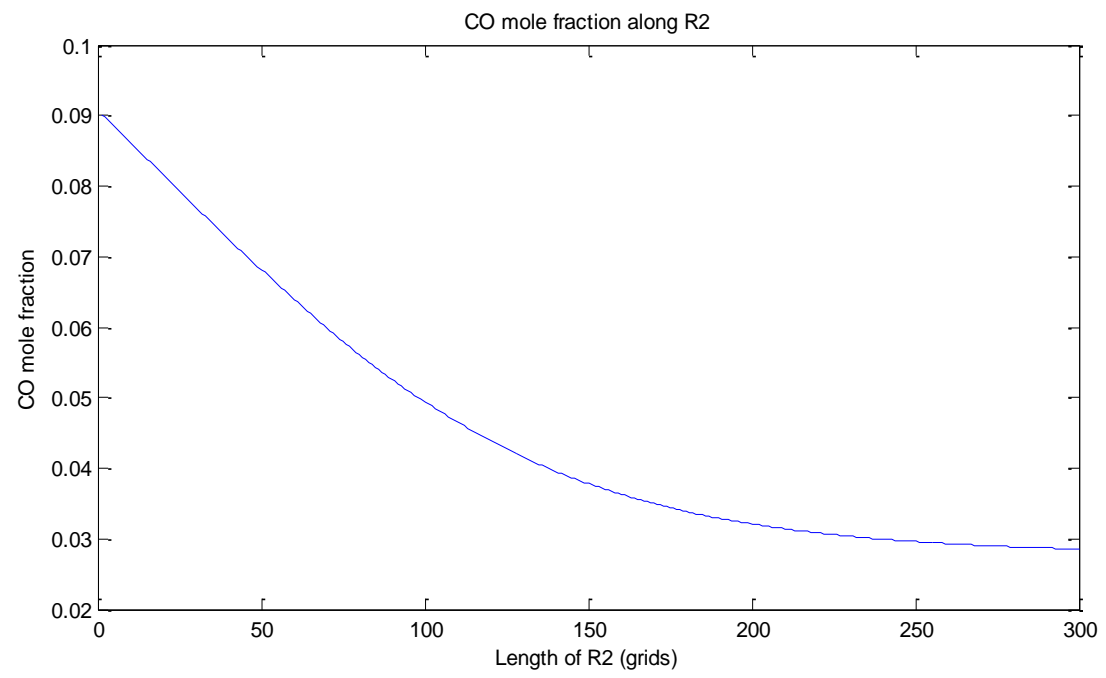

Figure 6.15: Profile of $\mathrm{CO}$ mole fraction along the length of reactor R2

As the WGS reaction reaches equilibrium towards the end of R2, mole fraction of $\mathrm{CO}$ changes negligibly towards the end of R2. The spatial profile of $\mathrm{CO}$ in $\mathrm{R} 2$ is shown in Figure 6.15.

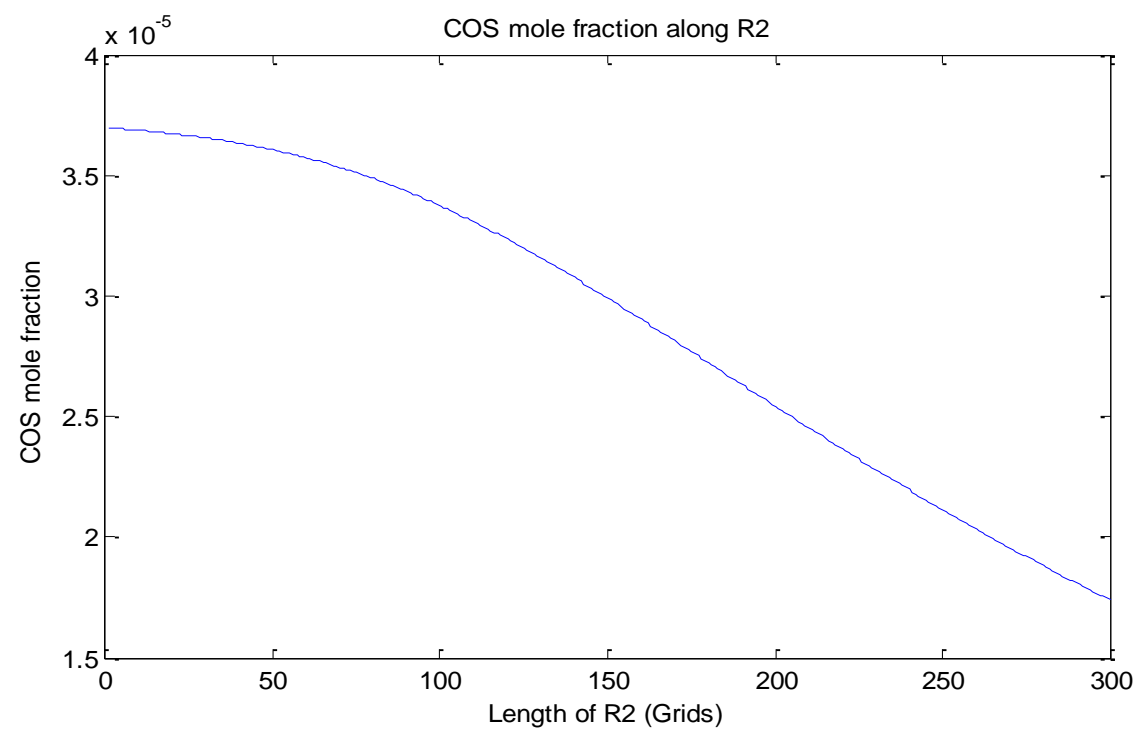

Figure 6.16: Plot of COS mole fraction along R2

The mole fraction of COS in the entering syngas stream fed to R1 is $1.38 \times 10^{-4}$. This reduces to $1.7 \times 10^{-5}$ at the end of R2 as can be seen in Figure 6.16. It should be noted that even though COS mole fraction at the inlet is small, it is important to convert it to $\mathrm{H}_{2} \mathrm{~S}$ so that it can be captured in the AGR unit for satisfying the overall emission requirements of sulfur. 


\subsection{Conclusions}

A 1-D dynamic model of a sour water gas shift reactor has been developed. The available experimental data for a sour WGS reactor have been reconciled to obtain consistent data. A simulation study under typical conditions of a sour WGS reactor as part of an IGCC plant is performed. For the range of inlet temperatures studied, i.e., 580-660 K, the COS conversion is found to be not affected by the feed temperature. In addition, even though an increase in the temperature results in slightly lower $\mathrm{CO}$ conversion, the reactor shows inverse response to a step increase in the syngas inlet temperature. At constant flow, as the steam-to-CO ratio increases, the $\mathrm{CO}$ conversion reaches a maximum at steam-to-CO ratio of about 4 while the COS conversion remains at its highest value for the range of steam-to-CO studied. It is observed that the $\mathrm{CO}$ and COS conversions are not significantly affected by the catalyst deactivation if the inlet temperature is sufficiently high.

A two-stage SWGSR is developed based on the single stage model. An inter-stage cooler is included to operate the second reactor at a lower temperature. Additional faults are simulated in the two-stage reactor system including change in porosity, activity and surface area. The control configuration is developed so as to operate it similar to an actual reactor. This model is later used for simulating the gasification island by integrating it with the SELEXOL unit. This will be discussed in Chapter 7, as part of the 2-tier sensor placement work. 


\section{FAULT SIMULATION IN PLANTS}

\subsection{Introduction}

In this work, potential faults are first identified by analyzing the information available in the open literature from the operating IGCC plants. Then the plant model is enhanced for simulating the faults. Due to the limitations in the Aspen Plus Dynamics software, for certain faults additional "dummy" equipment items were created. For example, for simulating the leakage fault, additional streams and additional valve(s) are needed to specify the leakage rate. Such changes in the flowsheets are done in a way so that convergence properties remain relatively unaffected.

The final step, before the fault is simulated, is the selection of potential measured variables. As each fault is simulated, the responses of the variables need to be recorded and saved. These data will be used by the sensor placement algorithm to identify potential sensor types and locations. However, there are a large number of potential measured variables. For example, the SELEXOL unit has over 39000 variables. Furthermore, each variable is recorded after every time interval of 1.8 seconds. Each fault is typically simulated for more than 2 hours until the model reaches a steady state. Therefore, without appropriate criteria for selection of variables, the size of the collected data can be intractable. 
The following guidelines are used in order to select these variables:

1. Instead of the molar flow, only the volumetric flow rate is recorded. This is because the volumetric flow rates are typically measured by the common sensors.

2. Across a heat exchanger (HE) the composition is not expected to change. Therefore, only pressure, flow rate and temperature are recorded.

3. Pressure and temperatures after splitter/mixers are not recorded.

4. Only pressures after valves or other pressure-drop devices are recorded.

5. The composition of only the gas stream exiting the flash vessel is recorded. The pressure and temperature of the vessel is recorded.

6. Levels in flash vessels are recorded.

7. Power outputs from turbines are recorded.

All equipment and streams are first individually analyzed to ensure that no redundant information is being recorded. Exceptions to the above guidelines are made for the equipment in which faults are simulated. For example, although the flow rate and composition across a HE are not expected to change, a rupture in the tubes of the HE may result in the mixing of the hot side and cold side fluids.

With the total number of candidate sensor placement variables brought down considerably, the historical data of selected variables are recorded for each fault simulation. In the current chapter, the process models of the important units in the IGCC plant are briefly described and the faults are simulated and discussed. The results for sensor placement, based on the fault simulation data, are also shown at the end of each section. The sensor placement algorithm is developed and executed by our collaborators at Texas Tech University. Some results from that work are reported here to provide an insight into the importance of fault simulation work. 


\subsection{Acid Gas Removal Plant - SELEXOL unit}

The SELEXOL technology used in the AGR unit uses chilled SELEXOL solvent. SELEXOL is a mixture of dimethyl ethers of polyethylene glycol (DEPG), i.e., $\mathrm{CH}_{3}\left(\mathrm{CH}_{2} \mathrm{CH}_{2} \mathrm{O}\right)_{n} \mathrm{CH}_{3}$, where 3 $\leq \mathrm{n} \leq 9$. SELEXOL is a physical solvent. The dual-stage SELEXOL unit absorbs $\mathrm{H}_{2} \mathrm{~S}$ in the first stage and $\mathrm{CO}_{2}$ in the second stage. The captured $\mathrm{CO}_{2}$ is recovered at three pressure levels and sent for compression. The $\mathrm{H}_{2} \mathrm{~S}$ that is thermally stripped from the loaded solvent is sent to the Claus unit for sulfur recovery. A fault in this unit may result in reduction of plant efficiency, or loss of expensive solvent or other species, or can lead to hazardous conditions.

\subsubsection{Model Development}

A schematic for the SELEXOL unit is shown in Figure 7.1.

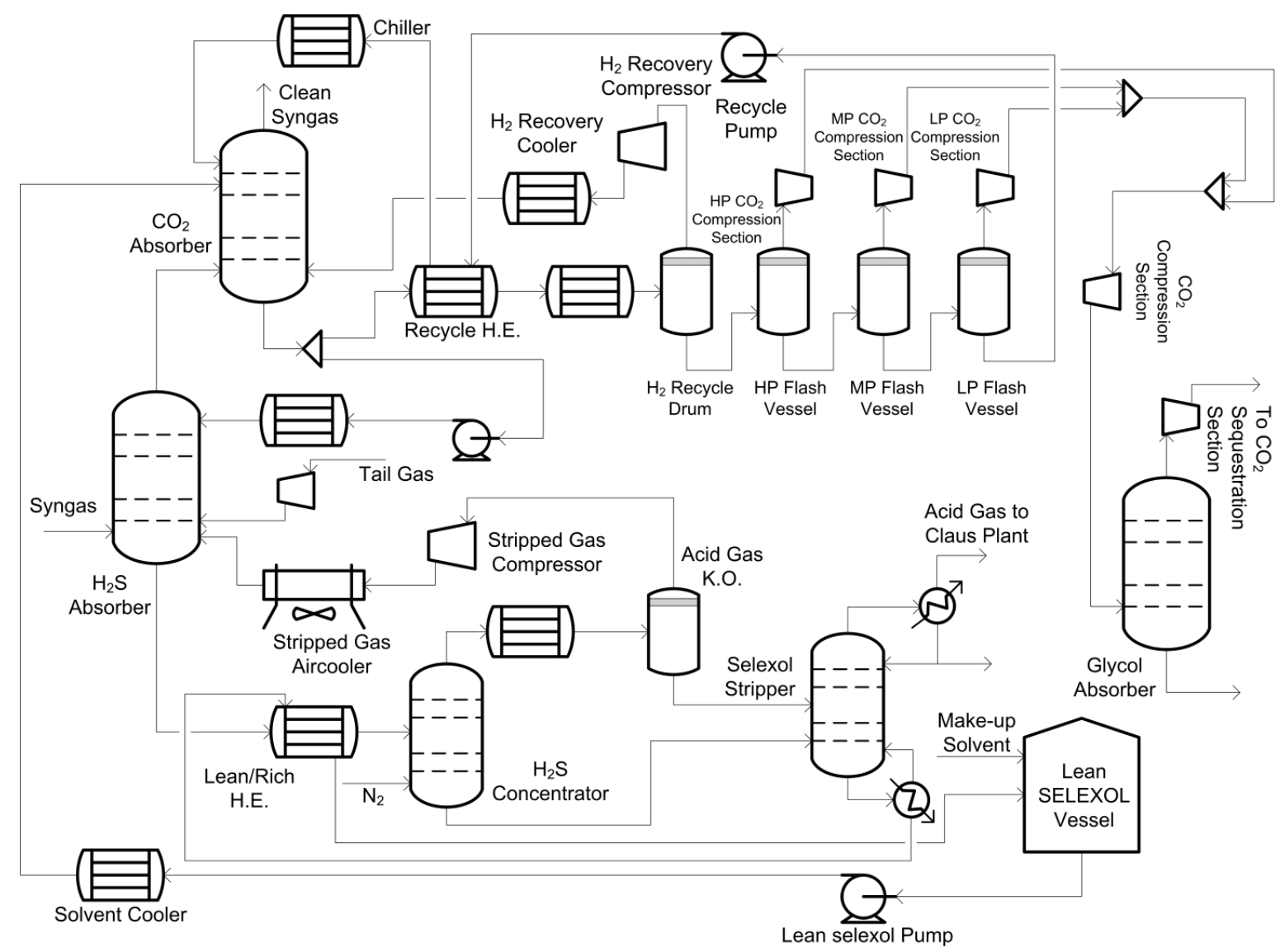

Figure 7.1: Schematic for SELEXOL unit 
In this process, the syngas is fed to the bottom of the $\mathrm{H}_{2} \mathrm{~S}$ absorber where the $\mathrm{CO}_{2}$-rich solvent from the bottom of the $\mathrm{CO}_{2}$ absorber is fed to the top of the column. The $\mathrm{H}_{2} \mathrm{~S}$-lean syngas enters the $\mathrm{CO}_{2}$ absorber where the lean solvent is fed at the top and partially regenerated semi-lean solvent is fed at the $5^{\text {th }}$ stage from the top.

A major portion of the solvent from the bottom of the $\mathrm{CO}_{2}$ absorber goes through a series of four flash vessels. In the first flash vessel, significant amount of the dissolved $\mathrm{H}_{2}$ is recovered from the solvent and sent back to the $\mathrm{CO}_{2}$ absorber. The solvent then passes through the high pressure (HP), medium pressure (MP) and low pressure (LP) flash vessels for recovering $\mathrm{CO}_{2}$. The recovered $\mathrm{CO}_{2}$ is sent to the compression unit for sequestration. The semi-lean solvent from the LP flash vessel is pumped back to the $\mathrm{CO}_{2}$ absorber. Rich solvent from the $\mathrm{H}_{2} \mathrm{~S}$ absorber is sent to the regenerator via a $\mathrm{H}_{2} \mathrm{~S}$ concentrator. Before introducing the solvent to the $\mathrm{H}_{2} \mathrm{~S}$ concentrator, the solvent is heated up in a lean/rich heat exchanger. The $\mathrm{CO}_{2}$-rich stream from the top of the $\mathrm{H}_{2} \mathrm{~S}$ concentrator is then recycled back to the $\mathrm{H}_{2} \mathrm{~S}$ absorber. In the stripper, the reboiler in combination with steam stripping is used to thermally regenerate the solvent. The $\mathrm{H}_{2} \mathrm{~S}$-rich gas from the top of the stripper reflux vessel goes to the Claus unit for further treatment. The lean solvent exchanges heat in the lean/rich heat exchanger, which is chilled using liquid ammonia, and is pumped back to the $\mathrm{CO}_{2}$ absorber. The make-up solvent is fed to the lean solvent to compensate for any loss of the solvent.

The design of the SELEXOL unit and development of the steady-state model in the Aspen Plus ${ }^{\circledR}$ and Aspen Plus Dynamics environment has been detailed by Bhattacharyya et al. (2010). The SELEXOL model is part of a plant wide IGCC model. For the current fault simulation work, the SELEXOL model is first isolated and solved for steady state. The control systems have been modified so as to ensure the process is stable in the range of operation of interest. Several structural modifications have been made, in order to simulate faults. 


\subsubsection{Fault Simulation}

Solids may be produced due to solvent degradation or corrosion, or may be entrained in the syngas. These solids can deposit on the trays of the absorbers and stripper of the Selexol unit leading to a decrease in the tray efficiency and an increase in the pressure drop. Another fault that can occur due to deposit of the solids is fouling in the heat exchangers. Tube leakage can result in product contamination, loss in the efficiency, and hazardous situations. Another type of leakage that is simulated is the leakage in the $\mathrm{H}_{2}$ recovery compressor suction line and leakages in flash vessels. A list of the faults simulated in the SELEXOL model is shown in Table 7.1.

Table 7.1: List of faults simulated in the SELEXOL unit

\begin{tabular}{|c|c|c|}
\hline Fault no. & $\begin{array}{c}\text { Fault } \\
\text { symbol }\end{array}$ & Description \\
\hline 1 & $F 1$ & Reduction in area of $13^{\text {th }}$ tray of $\mathrm{CO}_{2}$ absorber by $15 \%$ \\
\hline 2 & $F 2$ & Reduction in area of bottom $\left(15^{\text {th }}\right)$ tray of $\mathrm{CO}_{2}$ absorber by $15 \%$ \\
\hline 3 & F3 & Reduction in area of $23^{\text {rd }}$ tray of $\mathrm{H}_{2} \mathrm{~S}$ absorber by $15 \%$ \\
\hline 4 & $F 4$ & Reduction in area of bottom $\left(26^{\text {th }}\right)$ tray of $\mathrm{H}_{2} \mathrm{~S}$ absorber by $15 \%$ \\
\hline 5 & F5 & Reduction in area of $4^{\text {th }}$ tray of $\mathrm{H}_{2} \mathrm{~S}$ concentrator by $15 \%$ \\
\hline 6 & F6 & Reduction in area of bottom $\left(6^{\text {th }}\right)$ tray of $\mathrm{H}_{2} \mathrm{~S}$ concentrator by $15 \%$ \\
\hline 7 & F7 & Reduction in overall heat transfer coefficient of Lean/Rich H.E. by $15 \%$ \\
\hline 8 & F8 & $1 \%$ leakage in the $\mathrm{H}_{2}$ recovery compressor suction line \\
\hline 9 & F9 & $1 \%$ vapor leakage in $\mathrm{H}_{2}$ recovery flash drum \\
\hline 10 & F10 & $1 \%$ vapor leakage in $\mathrm{CO}_{2}$ high pressure flash drum \\
\hline 11 & F11 & $1 \%$ vapor leakage in $\mathrm{CO}_{2}$ low pressure flash drum \\
\hline 12 & $F 12$ & $1 \%$ vapor leakage in $\mathrm{CO}_{2}$ medium pressure flash drum \\
\hline 13 & F13 & Reduction in area of $8^{\text {th }}$ tray of SELEXOL stripper by $15 \%$ \\
\hline 14 & $F 14$ & Reduction in area of bottom $\left(11^{\text {th }}\right)$ tray of SELEXOL stripper by $15 \%$ \\
\hline
\end{tabular}




\subsubsection{Sensor Placement Results}

The fault simulation data are processed by the sensor placement algorithm. Three different algorithms are used in order to find a sensor network that can detect and resolve all faults at minimum cost. A list of the sensors that were selected by the algorithms as candidate sensor locations is provided in Table 7.2. Note that this list does not provide all the sensors that have responded to fault simulation, but only those that were selected by the algorithms based on the selection criteria.

Table 7.2: List of measured variables in SELEXOL process

\begin{tabular}{|c|c|c|}
\hline $\begin{array}{c}\text { Sensor } \\
\text { no. }\end{array}$ & Sensor type & Location \\
\hline s1 & Composition $\left(\mathrm{N}_{2}\right)$ & $7^{\text {th }}$ tray of SELEXOL stripper \\
\hline $\mathrm{s} 2$ & Composition $\left(\mathrm{NH}_{3}\right)$ & $10^{\text {th }}$ tray of SELEXOL stripper \\
\hline s3 & Temperature & Pure SELEXOL stream from SELEXOL stripper \\
\hline s4 & Temperature & $10^{\text {th }}$ tray of $\mathrm{CO}_{2}$ absorber \\
\hline s5 & Temperature & $1^{\text {st }}$ stage compression of $\mathrm{LP} \mathrm{CO}_{2}$ \\
\hline s6 & Flow rate & $\mathrm{CO}_{2}$ stream top of flash vessel at MP \\
\hline s7 & Flow rate & Make-up SELEXOL stream \\
\hline s8 & Composition $\left(\mathrm{H}_{2} \mathrm{~S}\right)$ & $10^{\text {th }}$ tray of SELEXOL stripper \\
\hline s9 & Temperature & $6^{\text {th }}$ tray of $\mathrm{H}_{2} \mathrm{~S}$ concentrator \\
\hline s10 & Temperature & $6^{\text {th }}$ tray of SELEXOL stripper \\
\hline s11 & Flow rate & $\mathrm{CO}_{2}$ stream from LP flash vessel to $1^{\text {st }}$ stage compressor \\
\hline s12 & Composition $\left(\mathrm{H}_{2} \mathrm{~S}\right)$ & $5^{\text {th }}$ tray of $\mathrm{CO}_{2}$ absorber \\
\hline s13 & Flow rate & $\mathrm{CO}_{2}$ stream from HP flash vessel to mix with MP stream \\
\hline s14 & Composition (COS) & $4^{\text {th }}$ tray of $\mathrm{H}_{2} \mathrm{~S}$ concentrator \\
\hline s15 & Temperature & $7^{\text {th }}$ tray of $\mathrm{CO}_{2}$ absorber \\
\hline s16 & Flow rate & $\mathrm{CO}_{2}$ stream from MP flash vessel to mix with LP stream \\
\hline s17 & Composition (SELEXOL) & $8^{\text {th }}$ tray of SELEXOL stripper \\
\hline s18 & Composition $\left(\mathrm{H}_{2} \mathrm{~S}\right)$ & $4^{\text {th }}$ tray of $\mathrm{CO}_{2}$ absorber \\
\hline s19 & Composition $\left(\mathrm{NH}_{3}\right)$ & $11^{\text {th }}$ tray of SELEXOL stripper \\
\hline s20 & Composition $\left(\mathrm{H}_{2} \mathrm{~S}\right)$ & Bottom stream of $\mathrm{H}_{2} \mathrm{~S}$ absorber fed to concentrator \\
\hline s21 & Temperature & Circulating SELEXOL stream from tank \\
\hline s22 & Composition $\left(\mathrm{H}_{2} \mathrm{~S}\right)$ & $8^{\text {th }}$ tray of SELEXOL stripper \\
\hline s23 & Composition $\left(\mathrm{NH}_{3}\right)$ & $9^{\text {th }}$ tray of SELEXOL stripper \\
\hline s24 & Temperature & SELEXOL mixing tank temperature \\
\hline s25 & Flow rate & $\mathrm{CO}_{2}$ stream from HP flash vessel to mix with MP stream \\
\hline
\end{tabular}


Table 7.3 shows the comparison of different algorithms for SELEXOL process. Although all faults are resolvable by all algorithms, there is a slight improvement in sensor network cost by applying fault evolution sequence (FES) and magnitude ratio (MR) compared to SDG. The last column in this table shows a null set, meaning that all the algorithms are able to resolve all faults.

It appears that flow and concentration sensors are mainly required. The FES algorithm uses one less temperature sensor than the SDG. Therefore, the sensor network cost is reduced. At low and medium MR threshold level, less temperature sensors are selected and the sensor network cost is reduced compared to FES and the combination of FES and MR takes advantage of both algorithms. At high MR threshold level, there is no improvement from SDG and the combination of FES and MR takes advantage of both algorithms.

Table 7.3: Results of applying different algorithms to SELEXOL process

\begin{tabular}{ccccc}
\hline Algorithm(s) & MR value level & Selected sensors & $\begin{array}{c}\text { Sensor } \\
\text { network cost }\end{array}$ & $\begin{array}{c}\text { Irresolvable } \\
\text { fault sets }\end{array}$ \\
\hline SDG & & $\mathrm{s} 1, \mathrm{~s} 2, \mathrm{~s} 3, \mathrm{~s} 4, \mathrm{~s} 5, \mathrm{~s} 6, \mathrm{~s} 7$ & 22.3 & {[]} \\
FES & s1,s7,s8,s9,10,s11 & 22.2 & {[]} \\
& Low & $\mathrm{s} 1, \mathrm{~s} 7, \mathrm{~s} 12, \mathrm{~s} 13$ & 22.0 & {[]} \\
MR & Medium & $\mathrm{s} 7, \mathrm{~s} 8, \mathrm{~s} 14, \mathrm{~s} 15, \mathrm{~s} 16$ & 22.1 & {[]} \\
& High & $\mathrm{s} 1, \mathrm{~s} 6, \mathrm{~s} 7, \mathrm{~s} 10, \mathrm{~s} 15, \mathrm{~s} 16, \mathrm{~s} 17$ & 22.3 & {[]} \\
& Low & $\mathrm{s} 7, \mathrm{~s} 11, \mathrm{~s} 18, \mathrm{~s} 19$ & 22.0 & {[]} \\
FES \& MR & Medium & $\mathrm{s} 7, \mathrm{~s} 8, \mathrm{~s} 11, \mathrm{~s} 20, \mathrm{~s} 21$ & 22.1 & {[]} \\
& High & $\mathrm{s} 7, \mathrm{~s} 10, \mathrm{~s} 22, \mathrm{~s} 23, \mathrm{~s} 24, \mathrm{~s} 25$ & 22.2 & {[]} \\
\hline
\end{tabular}




\subsection{Combined Cycle unit}

A schematic of the combined cycle power plant is shown in Figure 7.2.

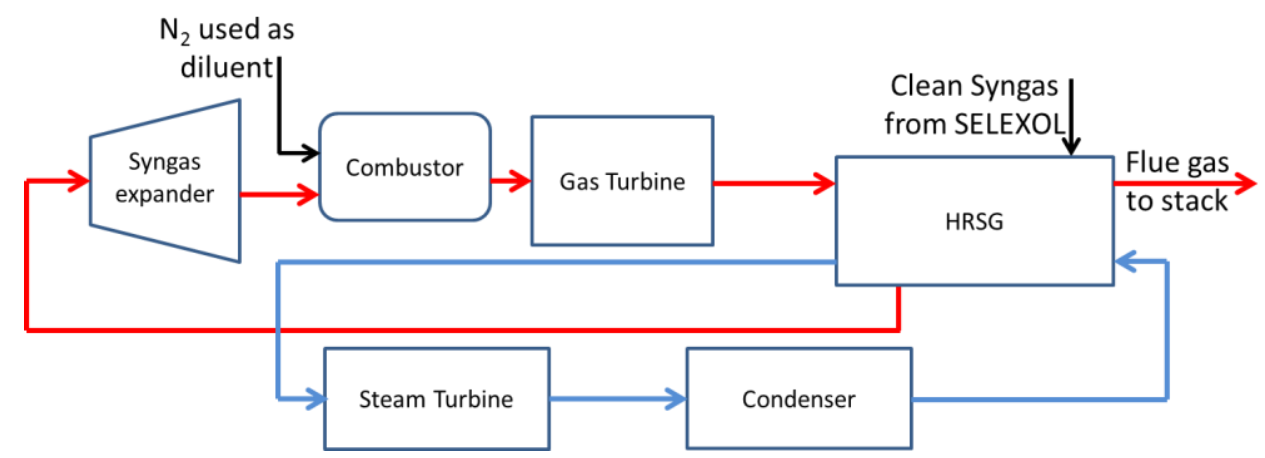

Figure 7.2: Schematic of the combined cycle power plant

Clean synthesis gas, exiting the SELEXOL unit is heated and mixed with $\mathrm{N}_{2}$, which is used as a diluent. After going through an expander, it is sent to the combustor of the GT. Hot exhaust gas from the GT is routed through the heat recovery steam generator to generate $1,800 \mathrm{psig}, 565^{\circ} \mathrm{C}$ super-heated HP steam, as well as reheat intermediate pressure (IP) steam to $565^{\circ} \mathrm{C}$ without supplemental firing. The HRSG also generates saturated HP steam (and possibly superheats steam as well) from gasification syngas cooling. The HP and IP superheated steam are routed to the ST to generate additional electric power. The combined cycle model from the work of Bhattacharyya et al. (2010) is used for this study and has been modified for simulating faults.

\subsubsection{Model Development}

The GT is simulated using turbine models available in the Aspen Plus library on the basis of the specifications of a GEE 7FB turbine. $\mathrm{N}_{2}$ is used as a diluent and is manipulated by a design specification so that the lower heating value (LHV) of the syngas fuel is reduced to $4.55 \mathrm{MJ} /$ $\mathrm{Nm}^{3}$ to keep the NOx concentrations in the ppmv range in the exhaust. The combustion air is compressed in an axial flow compressor which raises the pressure to about 1.65 MPa. When the flow of combustion air is manipulated, the GT combustor temperature is maintained at $1377^{\circ} \mathrm{C}$ with a specified heat loss equal to $1.5 \%$ of the lower heating value (LHV) of the syngas. The GT firing temperature is maintained at $1327^{\circ} \mathrm{C}$ by a design specification which manipulates the air flow rate to the combustor outlet gas before it reaches the first expansion stage. The air flow 
rates to the second and third expansion stages are maintained at predetermined values. The isentropic efficiencies of the GT are manipulated such that the exhaust temperature is maintained at $566^{\circ} \mathrm{C}$. The isentropic efficiencies of all the three stages are assumed to be equal. The flue gas goes to the heat recovery steam generator (HRSG) where steam is generated at three pressure

levels. The flue gas is used to superheat the HP steam generated both in the HRSG evaporator and in the radiant syngas cooler before it finally exits the system at $132^{\circ} \mathrm{C}$, well above the cold end corrosion temperature.

The steam cycle generates steam from the flue gas and other process streams at three pressure levels. The minimum temperature approach is considered to be $10^{\circ} \mathrm{C}$ in this study. $\mathrm{HP}$ steam, generated at $12.4 \mathrm{MPa}$ and $538^{\circ} \mathrm{C}$, is mainly used for generating power in the HP steam turbine (ST). IP steam is used for generating power, as well as in the reboilers. LP steam generated in the HRSG is mainly used for heating process streams and in the reboilers. Condensate at the outlet of the surface condenser and from the LP steam circuit and flash steam from the HP blow down drum are sent to the deaerator. The boiler feed water (BFW) at the outlet of the deaerator is pumped at various pressure levels for generating HP, IP, and LP steam. The HP stream is heated and sent to the RSC and HRSG. It is then superheated and sent to the HP turbine. IP BFW passes through the economizer and evaporator to generate IP steam which is sent to the IP turbine. The LP split of the BFW is used to generate LP and IP steam. The exit temperature of the flue gas above the cold end corrosion temperature is maintained by manipulating the flow of the BFW that goes to the LP steam evaporator.

\subsubsection{Fault Simulation}

The faults simulated in the combined cycle island include leakage at several locations, fouling within a few heat exchangers and an increased loss of heat through the combustor. Since the turbines itself are highly advanced and consist of several inbuilt sensors that detect and report any deviation from operation immediately, faults in these units have not been simulated. The leakage faults are mainly considered for the heat exchangers where high pressure differences exist between the shell and tube sides. Fouling is a concern within the heat exchangers as well. These faults are modeled similar to the methods mentioned earlier for the SELEXOL unit. The GT combustor has insulation to prevent heat loss to the environment. However, the insulation 
might get damaged in the course of operation and this can be modeled by increasing the heat loss in the GT combustor block.

The combined cycle section was segregated from the plant-wide model developed in the works of Bhattacharyya et al. (2010). The faults to be simulated in the combined cycle unit were identified. The faults that were selected and implemented are shown below. With the exception of Fault E, all faults are simulated with a ramp function of 1 hour duration initiated after half an hour of simulation.

\section{A) Leakage from the high pressure steam flash vessel}

Steam at very high temperature and pressure is produced in a steam generator unit using the heat from hot flue gas. The steam produced is then sent to the high pressure steam turbine. A leakage in the high pressure steam generator can cause mixing of the steam with the flue gas which could build up in the steam cycle.

\section{B) Leakage within a HE between syngas and steam streams}

A leakage fault can occur in the heat exchanger that is used to heat in the hydrogen rich syngas from the SELEXOL plant before being sent to the combustor. The steam is at higher pressure and a rupture in the tube can result in steam entering the gas cycle. This can negatively impact the combustion process and the power generated in the gas turbines.

\section{C) Leakage within a condenser between steam and water streams}

Usually a composition sensor placed at the one of the streams exiting the HE would be able to detect any leakage taking place within the HE. However, if the heat transfer involves two streams of the same material, a leak may become difficult to detect. A leak fault is simulated in the heat exchanger that uses cooling water to cool steam.

D) Fouling simulated as loss of area within HE

A fouling fault is simulated in the heat exchanger used to cool the flue gas from the combustor using steam. The combustor flue gas can have particulate matter entrained from the $\mathrm{N}_{2}$ stream that can deposit on the walls of the HE. This fault is simulated by decreasing the surface area of the HE. 
E) Increase in heat loss from the combustor

The combustor operates at high temperature and has insulation to limit the heat loss to the environment. However, the insulation may get damaged in course of operation. This fault is modeled by introducing a step change in the heat loss from the GT combustor block after half an hour of simulation time.

F) Leakage within a HE between combusted syngas and steam stream.

A leakage in an IP steam generator HE is simulated where heat from the flue gas at almost atmospheric pressure is used to generate steam. It is desired to observe if a sensor can be found to differentiate faults in heat exchangers operating at different pressures.

G) Fouling simulated as loss of area within HE

This fault is simulated in the same HE as Fault B. In the SELEXOL process, there can be some foaming or formation of undesirable chemicals on the trays of the absorbers. These materials can be carried by the syngas and can be deposited on the heat exchanger surfaces thereby reducing the surface area for heat exchange. 


\subsubsection{Sensor Placement Results}

The above faults in the combined cycle power plant are simulated at three severity levels. The data for the 21 faults were compiled and sent to TTU for sensor placement studies. The severity of the faults and their levels are shown in Table 7.4.

Table 7.4: Faults at different severity level simulated in the combined cycle model

\begin{tabular}{ccccc}
\hline Fault type & Fault \# & $\begin{array}{c}\text { Fault } \\
\text { sim }\end{array}$ & Duration & Severity \\
\hline A & $1-3$ & Ramp & 1 hour & $0.5 \%, 1 \%, 2 \%$ leak valve opening \\
B & $4-6$ & Ramp & 1 hour & $0.1 \%, 0.2 \%, 0.4 \%$ leak valve opening \\
C & $6-9$ & Ramp & 1 hour & $5 \%, 10 \%, 20 \%$ leak valve opening \\
D & $9-12$ & Ramp & 1 hour & $80 \%, 90 \%, 95 \%$ area available \\
E & $12-15$ & Step & - & $90 \%, 95 \%, 98 \%$ of original heat loss \\
F & $15-18$ & Ramp & 1 hour & $0.05 \%, 0.2 \%, 0.5 \%$ of leak valve opening \\
G & $18-21$ & Ramp & 1 hour & $85 \%, 90 \%, 95 \%$ of area available \\
\hline
\end{tabular}

Faults at three severity levels, namely high, medium and low level, are considered. It is expected that the effect of the high severity level should be captured by the algorithm easily. The low level faults might be the most difficult to be resolved by the algorithm. It was desired to observe what level of fault severity can be detected by the sensor network and the minimum number of sensors required to detect them.

736 variables pre-selected as candidate sensor placement variables and their historical data are recorded for the sensor placement algorithm. These variables include temperature, pressure, flow, concentration, power and level. Table 7.5 shows the weight and threshold value for each type of variables used in the algorithm. 
Table 7.5: Weight and threshold of each variable in the sensor placement algorithms

\begin{tabular}{ccc}
\hline Variable type & Weight & Threshold \\
\hline Temperature & 0.1 & $1 \mathrm{~F}$ \\
Level & 1 & 1 inch \\
Flow & 1 & $3 \%$ \\
Power & 0.1 & $3 \%$ \\
Concentration & 10 & 0.01 \\
(mole fraction) & & \\
Pressure & 0.5 & $2 \mathrm{psi}$ \\
\hline
\end{tabular}

Table 7.6 and Table 7.7 show the results of the different algorithms for fault resolution of combined cycle. The location and number of sensors selected by the FES differ from that of the SDG algorithm in Table 7.6. The cost of the final sensor network in the FES is lower, although it can be seen that both algorithms are not able to differentiate between two set of the faults.

Faults 8 and 9 are two severities of a leak in a heat exchanger resulting in the mixing of steam and water. Although this fault is expected to be difficult to detect and resolve, the two algorithms are able to resolve this fault at a lower fault severity. Faults 14 and 15 may be difficult to resolve between because of the closeness of their severities.

Table 7.6: SDG and FES algorithms results

\begin{tabular}{cccc}
\hline Algorithm(s) & \# Sensors & $\begin{array}{c}\text { Sensor network } \\
\text { cost }\end{array}$ & $\begin{array}{c}\text { Irresolvable } \\
\text { fault sets }\end{array}$ \\
\hline SDG & 13 & 5.8 & {$[8,9][14,15]$} \\
FES & 12 & 4.8 & {$[8,9][14,15]$} \\
\hline
\end{tabular}

Table 7.7 shows that the MR algorithm can also resolve between faults [8,9], but only at the medium ratios of range 1.3 - 1.8. The MR algorithm is able to compare the magnitude of change in the sensor readings as an additional resolution feature in contrast to the other methods that are 
strictly qualitative. However, its ability also depends upon the candidate sensor types and locations selected/available. It is also found that the sensor network cost is sensitive to the MR threshold value in this range.

Table 7.7: MR algorithm results

\begin{tabular}{ccc}
\hline Algorithm & $\begin{array}{c}\text { MR threshold } \\
\text { value }\end{array}$ & $\begin{array}{c}\text { Irresolvable fault } \\
\text { sets }\end{array}$ \\
\hline \multirow{3}{*}{ MR } & $1.1-1.2$ & {$[8,9][14,15]$} \\
& $1.3-1.8$ & {$[14,15]$} \\
& $1.9-3$ & {$[8,9][14,15]$} \\
\hline
\end{tabular}

Figure 7.3, shows the number of irresolvable faults and the corresponding sensor network cost for different MR threshold values. It can be seen that for low and medium MR range, the cost of the sensor network selected is lower than that of the high MR range.
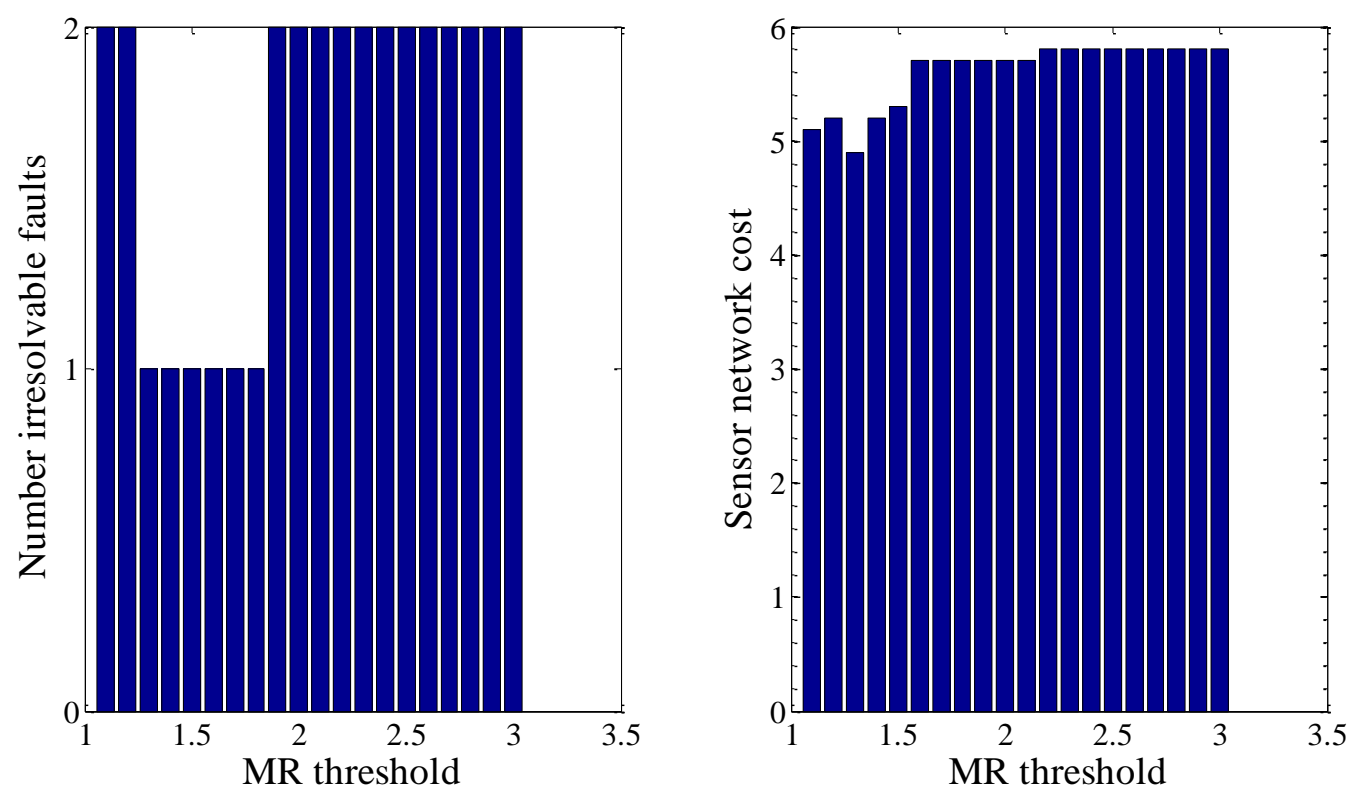

Figure 7.3: Number of irresolvable faults (left) and the corresponding sensor network cost (right) at different MR threshold values 


\subsection{Gasification Island - Two Tier Sensor Placement}

As mentioned earlier one of the objectives of the project is to study the efficacy of employing a two-tier sensor placement method by exploiting synergies between the sensor networks selected at two levels. To do this, the simultaneous simulation of the equipment level and the plant level is required. However, the SWGSR model has been developed using MATLAB while Aspen Plus Dynamics is used to simulate the SELEXOL unit. To simulate the gasification island using these models, a cross-platform simulation is required where the information between the two software needs to be exchanged and the two models need to be synchronized.

\subsubsection{Model Development}

The schematic for the two-stage SWGSR system shown in Figure 6.2 is redrawn as Figure 7.4.

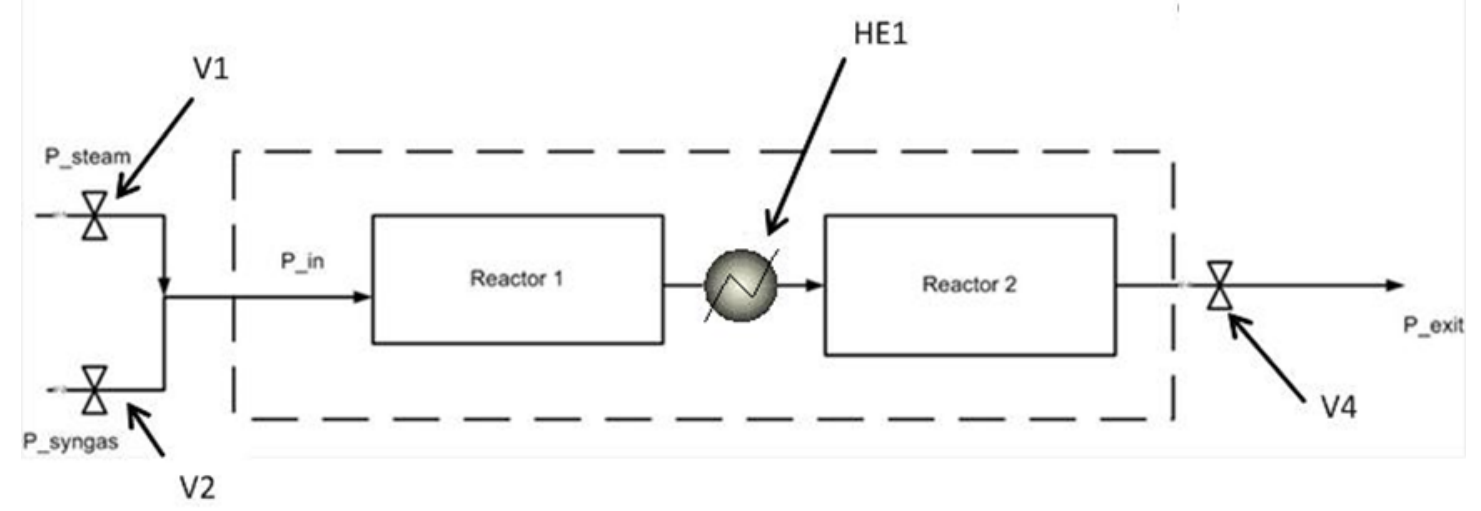

Figure 7.4: Schematic representation of the Sour Water Gas Shift Reactor system developed in MATLAB

The inlet variables for the SELEXOL unit are initialized with the outlet variables of the SWGSR unit and the steady-state model is converged. This is done to make it easy to obtain initial convergence when the two models are integrated.

The integration of the two models is done using SIMULINK. An Aspen Modeler Block can be used to export variables from the "workspace" in MATLAB and also to import variables from Aspen Plus Dynamics (APD). 
The coupling is done across the valve V4 in MATLAB and the first valve in the SELEXOL plant. The valve coefficients and the valve opening of the inlet valve to the SELEXOL unit are kept exactly the same as the valve V4 in MATLAB. The two valves are treated as though they were the same valve in an integrated flowsheet. Due to the coupled pressure-flow dynamics of the entire plant, the boundary conditions for pressure at the output of the MATLAB model and the pressure at the input of the APD models are not static, but dynamic and are thus synchronized.

The MATLAB solver first solves for a fixed time step using fixed inlet and outlet conditions. This time span, along with the temperature, pressure and composition before valve V4, are sent to the APD model via SIMULINK. These variables are treated as the input to the inlet stream in the SELEXOL model. The APD model is solved for the same time step, and an updated value of pressure after the inlet valve is sent back to MATLAB via SIMULINK. Finally, the exit pressure in the MATLAB simulation is updated, and the simulation is run once again.

A schematic of the exchange of information across the platforms is shown in Figure 7.5.

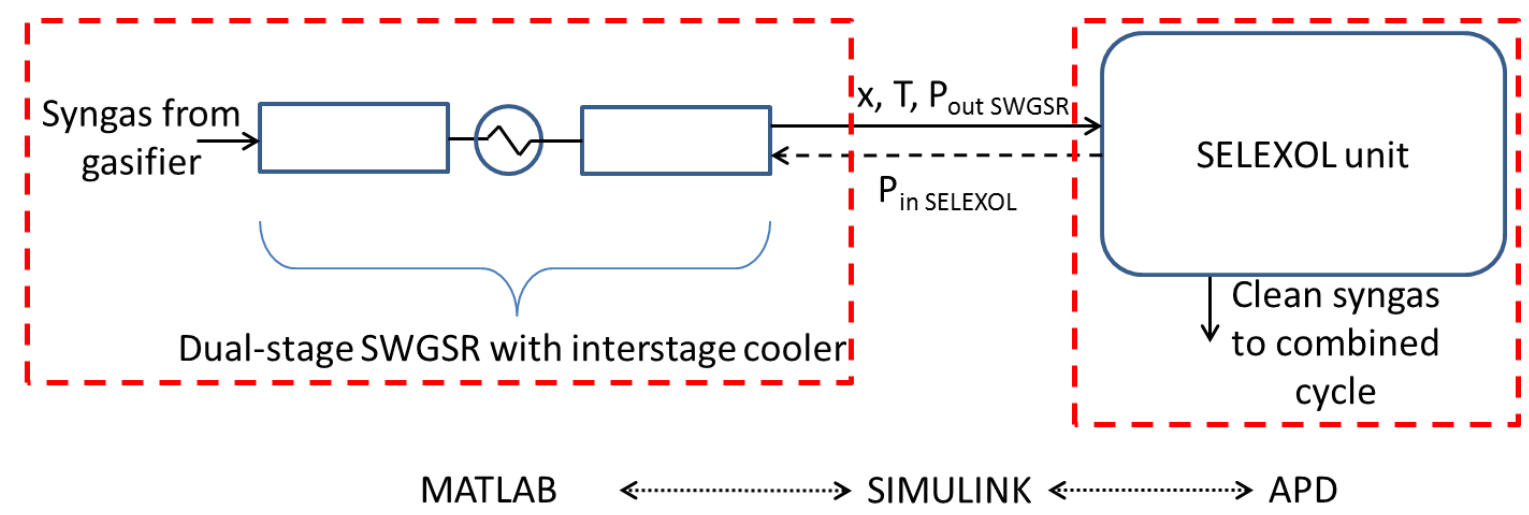

Figure 7.5: Exchange of information between the SWGSR in MATLAB and the SELEXOL model in APD

This process goes on for each time step until the end of the fault simulation. Through the process, the variables are recorded on both, the MATLAB side and the APD side. 


\subsubsection{Fault Simulation - Multiple Severity Faults}

For the fault simulation on the SELEXOL side of the gasification island, different faults are selected as compared to Section 7.2.2. The new faults simulated are as follows.

- $\underline{\mathrm{CO}}_{2}$ Absorber fault: simulated by reducing the $15^{\text {th }}$ tray area as syngas enters from bottom.

- $\underline{\mathrm{H}}_{2} \underline{\mathrm{S} \text { absorber fault: }}$ simulated by reducing the $26^{\text {th }}$ tray area. This tray is in the vicinity of stream coning from SWGSR, stream rich with $\mathrm{H}_{2} \mathrm{~S}$ coming from SELST and gas turbine outlet containing $\mathrm{CO}_{2}$ and $\mathrm{H}_{2}$. Possible reasons for this fault can be sulfur deposition or ash/soot carried along SWGSR.

- $\underline{\mathrm{H}}_{2} \underline{\mathrm{S} \text { concentrator fault: }}$ simulated by reducing the $5^{\text {th }}$ tray area. $\mathrm{N}_{2}$ stream enters at this tray. Possible reason for this fault can be due to particulate matter entrained in it.

- SELEXOL stripper fault: simulated by reducing the $6^{\text {th }}$ tray area. This is the feed tray for stream coming from $\mathrm{H}_{2} \mathrm{~S}$ concentrator. Deposit of particulate matters or other degradation products can lead to this fault.

- The heat exchanger H2SSTRBT is an important heat exchanger that heats the stream coming from the bottom of the $\mathrm{H}_{2} \mathrm{~S}$ absorber and sends it to the $\mathrm{H}_{2} \mathrm{~S}$ concentrator using the lean solvent stream.

- The heat exchanger PRCRE is a heat exchanger that is used to cool the solvent stream to the $\mathrm{CO}_{2}$ absorber. 
Multiple severities of these faults are simulated and are shown in Table 7.8.

Table 7.8: Faults simulated on the SELEXOL side of the integrated model

\begin{tabular}{cr}
\hline Fault & Description \\
\hline $\mathrm{F}_{1-3}$ & Reduction in the area of the bottom $\left(15^{\text {th }}\right)$ tray of the $\mathrm{CO}_{2}$ absorber $\times 3$ \\
$\mathrm{~F}_{4-6}$ & Reduction in the area of the bottom $\left(26^{\text {th }}\right)$ tray of the $\mathrm{H}_{2} \mathrm{~S}$ absorber $\times 3$ \\
$\mathrm{~F}_{7-9}$ & Reduction in the area of the bottom $\left(5^{\text {th }}\right)$ tray of the $\mathrm{H}_{2} \mathrm{~S}$ concentrator $\times 3$ \\
$\mathrm{~F}_{10-12}$ & Reduction in the area of the bottom $\left(6^{\text {th }}\right)$ tray of the SELEXOL stripper $\times 3$ \\
$\mathrm{~F}_{13-15}$ & Leakage fault at start of heat exchanger H2SSTRBT $\times 3$ \\
$\mathrm{~F}_{16-18}$ & Leakage fault at end of heat exchanger H2SSTRBT $\times 3$ \\
$\mathrm{~F}_{19-21}$ & Fouling fault simulated in heat exchanger H2SSTRBT $\times 3$ \\
$\mathrm{~F}_{22-24}$ & Fouling fault simulated in heat exchanger PRCRE $\times 3$ \\
\hline
\end{tabular}

A more detailed approach to simulate a fault at different locations within the same equipment is developed while simulating Fault $\mathrm{F}_{13-15}$ and $\mathrm{F}_{16-18}$. Both faults are simulated in the $\mathrm{HE}$ H2SSTRBT, however the configurations are different. Fault $F_{13-15}$ is simulated in the following manner as shown in Figure 7.6

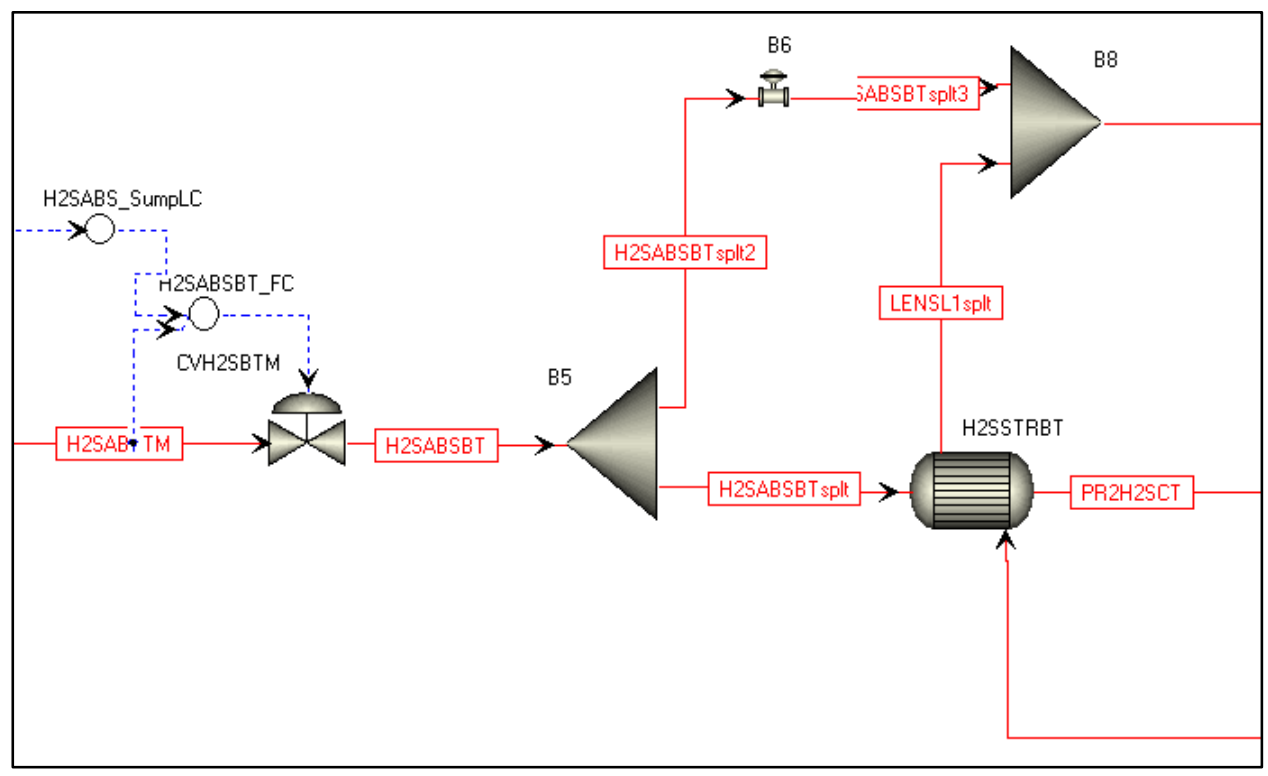

Figure 7.6: HE configuration 1: leak simulated as mixing of inlet high pressure stream into outlet low pressure stream

Fault $\mathrm{F}_{16-18}$ is simulated as shown in Figure 7.7. 


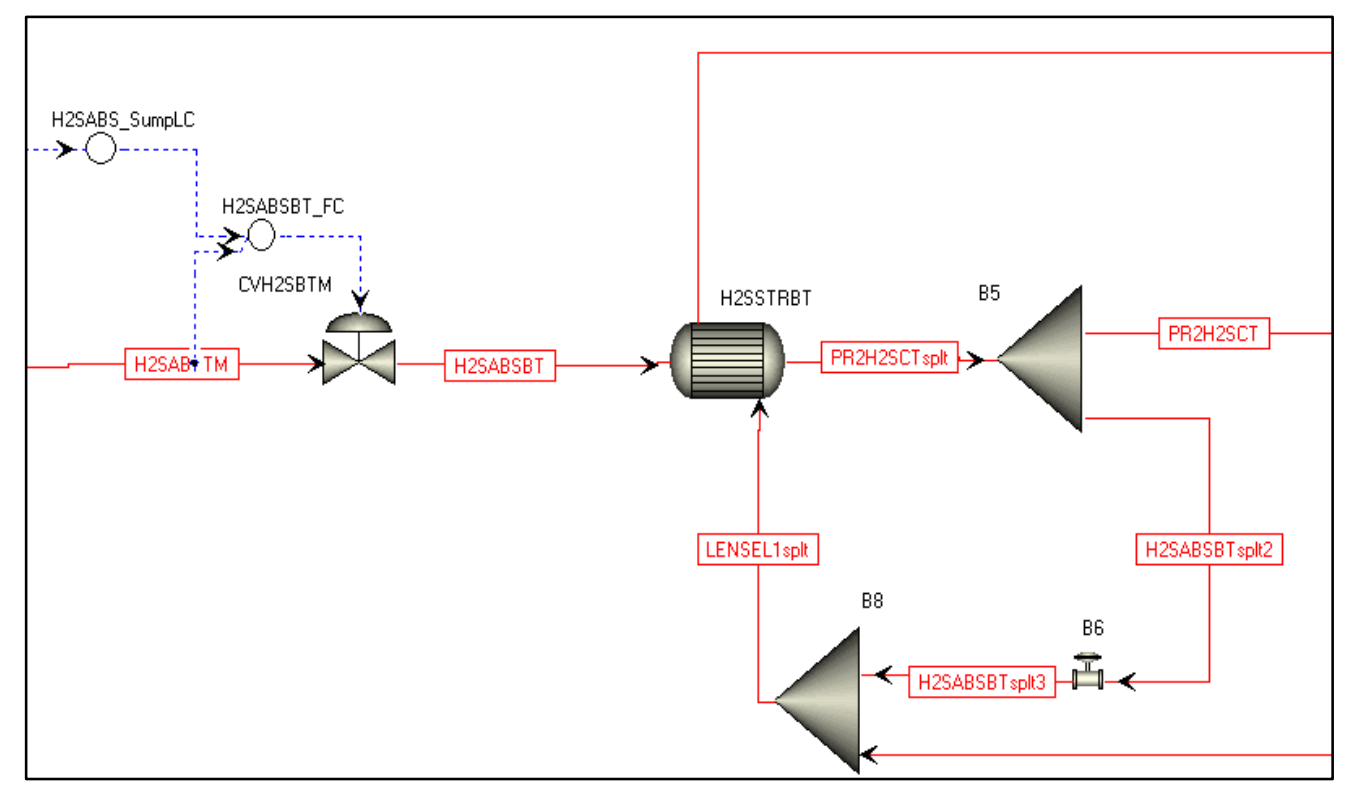

Figure 7.7: HE configuration 2: leak simulated as mixing of outlet high pressure stream into inlet low pressure stream

The purpose of simulating the leakage fault in two separate ways is to assess whether the sensor network can give information of the location of the leakage within the heat exchanger. In fault $\mathrm{F}_{13-15}$, the leakage has taken place closer to the entrance of the tubes while in fault $\mathrm{F}_{16-18}$ it is simulated as if the leakage has taken place towards the outlet of the tube side. This would bring key information into the sensor placement study if it can detect the location within single equipment while considering plant wide faults. Fault $\mathrm{F}_{22-24}$ is to see whether a sensor network can be found that can distinguish between a leakage fault and a fouling fault within the same equipment.

On the SWGSR side of the integrated model a total of 17 faults were simulated i.e. three instances of six faults discussed in Section 6.5.1.1 (except for fault $\mathrm{F}_{31-32}$ due to data problems). These are shown below in Table 7.9. 
Table 7.9: Faults simulated on the SWGSR side of the integrated model

\begin{tabular}{ccccc}
\hline Fault \# & Description & Fault type & Duration & Severity \\
\hline $\mathrm{F}_{25-27}$ & Porosity reduction ( $1^{\text {st }}$ reactor) & Ramp & $25 \mathrm{~min}$ & $2 \%, 5 \%, 10 \%$ \\
$\mathrm{~F}_{28-30}$ & Porosity reduction (both reactors) & Ramp & $25 \mathrm{~min}$ & $2 \%, 5 \%, 10 \%$ \\
$\mathrm{~F}_{31-32}$ & Activity reduction ( $1^{\text {st }}$ reactor) & Ramp & $25 \mathrm{~min}$ & $2 \%, 5 \%$ \\
$\mathrm{~F}_{33-35}$ & Activity reduction (both reactors) & Ramp & $25 \mathrm{~min}$ & $2 \%, 5 \%, 10 \%$ \\
$\mathrm{~F}_{36-38}$ & Surface area reduction ( $1^{\text {st }}$ reactor) & Ramp & $25 \mathrm{~min}$ & $2 \%, 5 \%, 10 \%$ \\
$\mathrm{~F}_{39-41}$ & Surface area reduction (both reactors) & Ramp & $25 \mathrm{~min}$ & $2 \%, 5 \%, 10 \%$ \\
\hline
\end{tabular}

\subsubsection{Results - Faults Simulation}

Before simulating the faults in the gasification island, it is important to implement the control configuration as would be expected in an actual operating plant. In the WGS reactor system, the syngas flowrate is maintained for producing the desired amount of power by the integrated gasification combined cycle (IGCC) plant. In addition, the $\mathrm{CO} / \mathrm{H}_{2} \mathrm{O}$ ratio at the inlet of the WGS reactor system is maintained by manipulating the steam flowrate to the reactors. These two controllers have been coded in MATLAB for manipulating valves V1 and V2 shown in Figure 7.4. The controllers were then tuned for satisfactory response.

The integrated system is used to simulate some typical faults. As an example, the results due to change in porosity of the first reactor, R1, will be presented below. This fault is expected to happen in a WGS reactor system as part of an IGCC plant since tar or soot that are generated in the gasifier but can escape the scrubber, can enter the reactor and clog the pores of the catalyst. As a result of this, the reaction rate can decrease. For this fault, it is assumed that the unwanted material is captured by the first reactor alone, and thus only the porosity of R1 is decreased. This is done at a rate of $25 \%$ decrease in porosity over a period of 12 hours. It should be noted that in real-life, such faults can happen over much longer period of time, but here a much faster rate is 
considered in order to study the capability of the integrated models. The results at the end for the first hour are shown below.

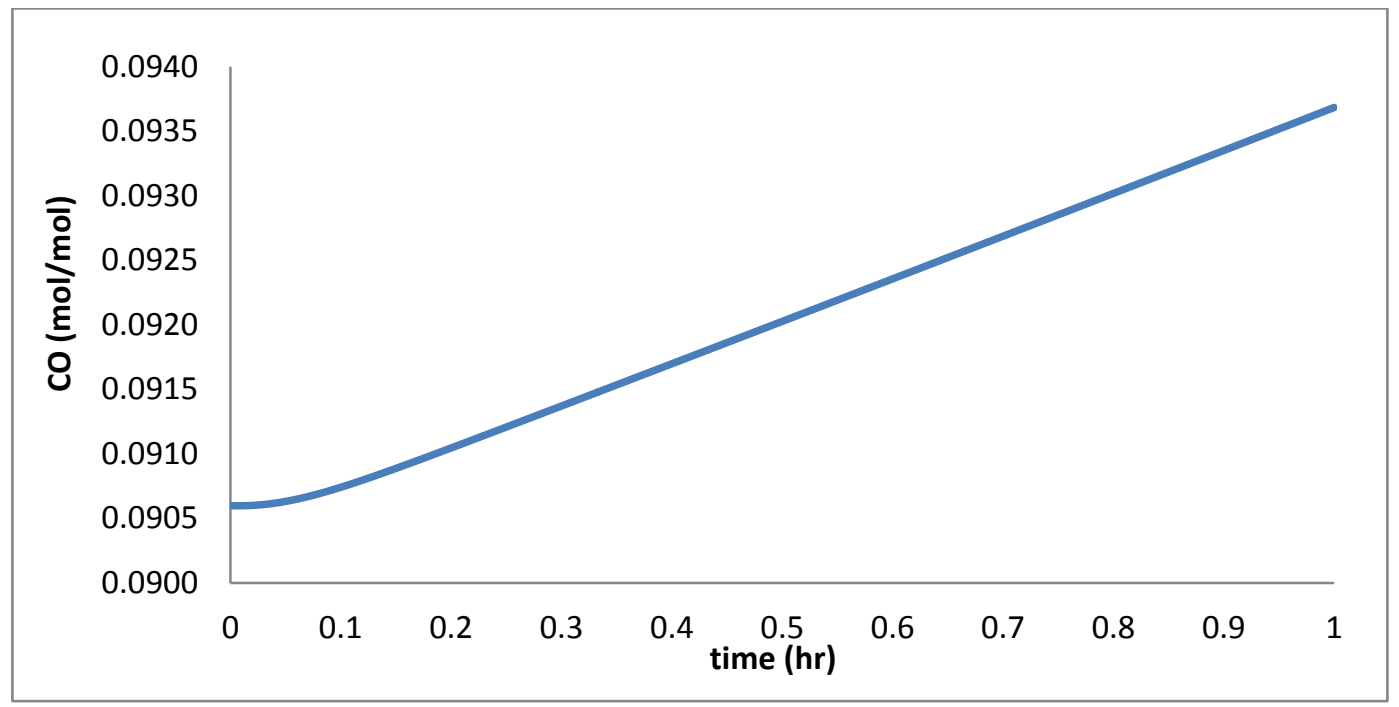

Figure 7.8: $\mathrm{CO}$ at the end of $\mathrm{R} 1$ as a result of a ramp decrease in the porosity

Figure 7.8 shows that $\mathrm{CO}$ composition at the end of $\mathrm{R} 1$ increases as time progresses. As the catalyst pores get clogged and the porosity decreases, the extent of WGS reaction reduces, and thus the amount of $\mathrm{CO}$ consumed reduces.

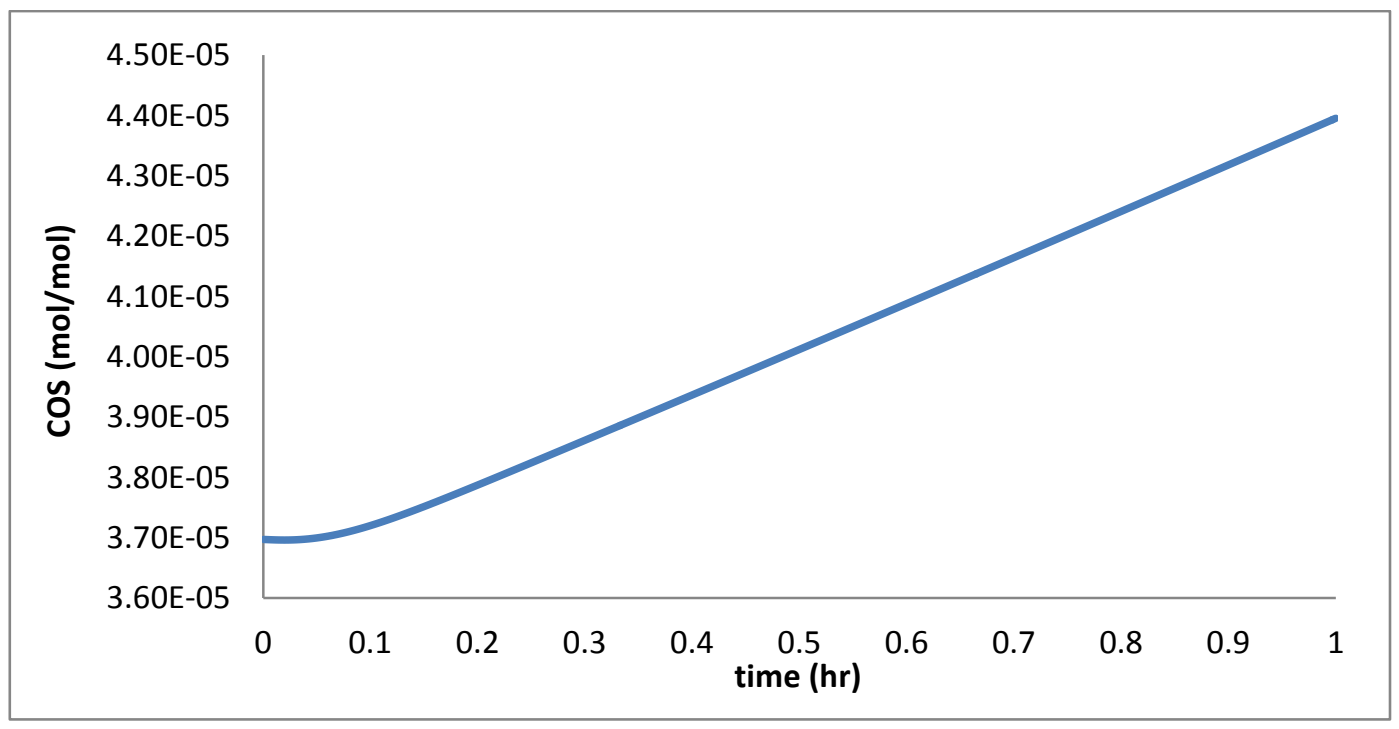

Figure 7.9: COS at the end of R1 as a result of a ramp decrease in porosity 
The rate of COS hydrolysis also gets affected due to the fault. The amount of COS converted reduces due to the reduction in porosity. Therefore, the COS mole fraction at the end of the reactor R1, increases, as can be seen from Figure 7.9.

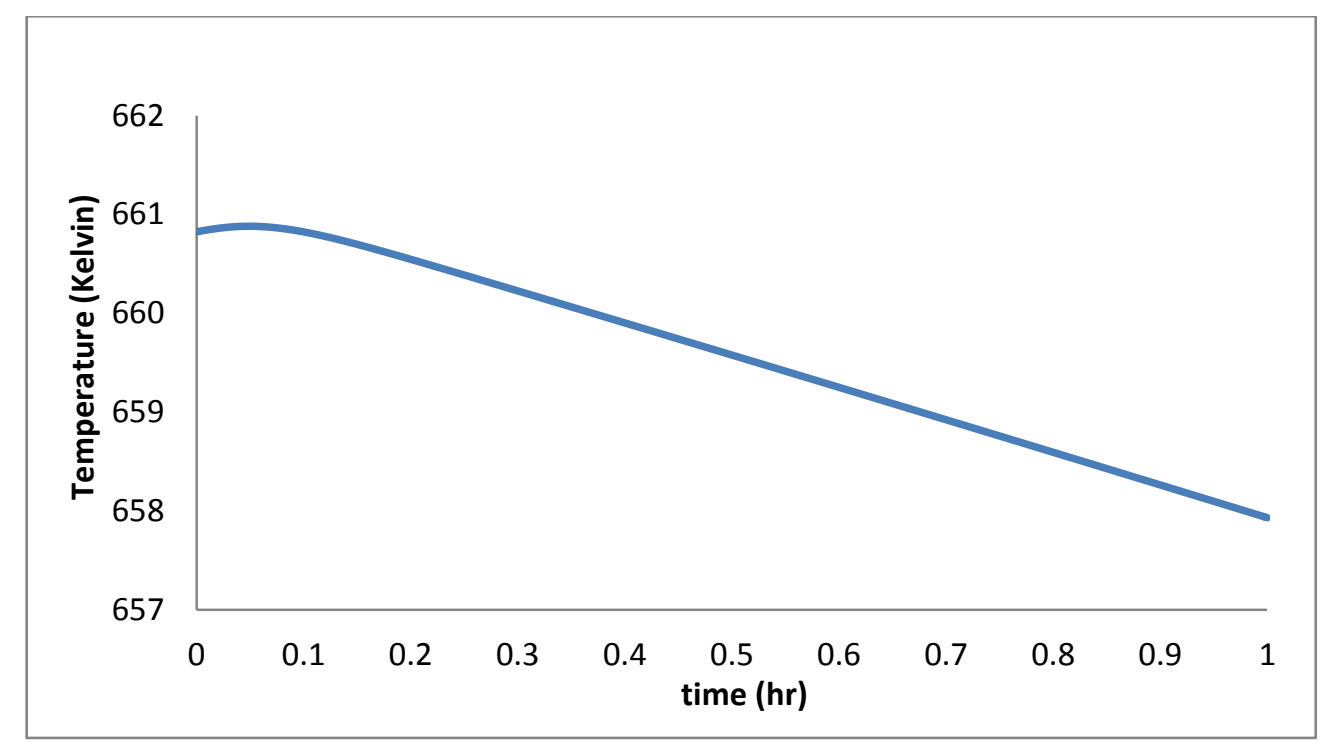

Figure 7.10: Temperature at end of $\mathrm{R} 1$ as a result of a ramp decrease in porosity

The WGS reaction is an exothermic reaction. The reaction does not reach equilibrium in the first reactor. As the extent of reaction decreases in reactor $\mathrm{R} 1$, the temperature at the exit also reduces. This can be seen in Figure 7.10.

Allowances have been provided in the design of the second reactor, R2, to accommodate acceptable deterioration in the performance of R1. Due to lower extent of WGS reaction in R1, the partial pressure of $\mathrm{CO}$ at the inlet of $\mathrm{R} 2$ increases. As a result, higher conversion of $\mathrm{CO}$ takes place in R2. The WGS reaction still approaches equilibrium, but it does so at different conditions as compared to what it had prior to the fault due to changes in the inlet conditions. 


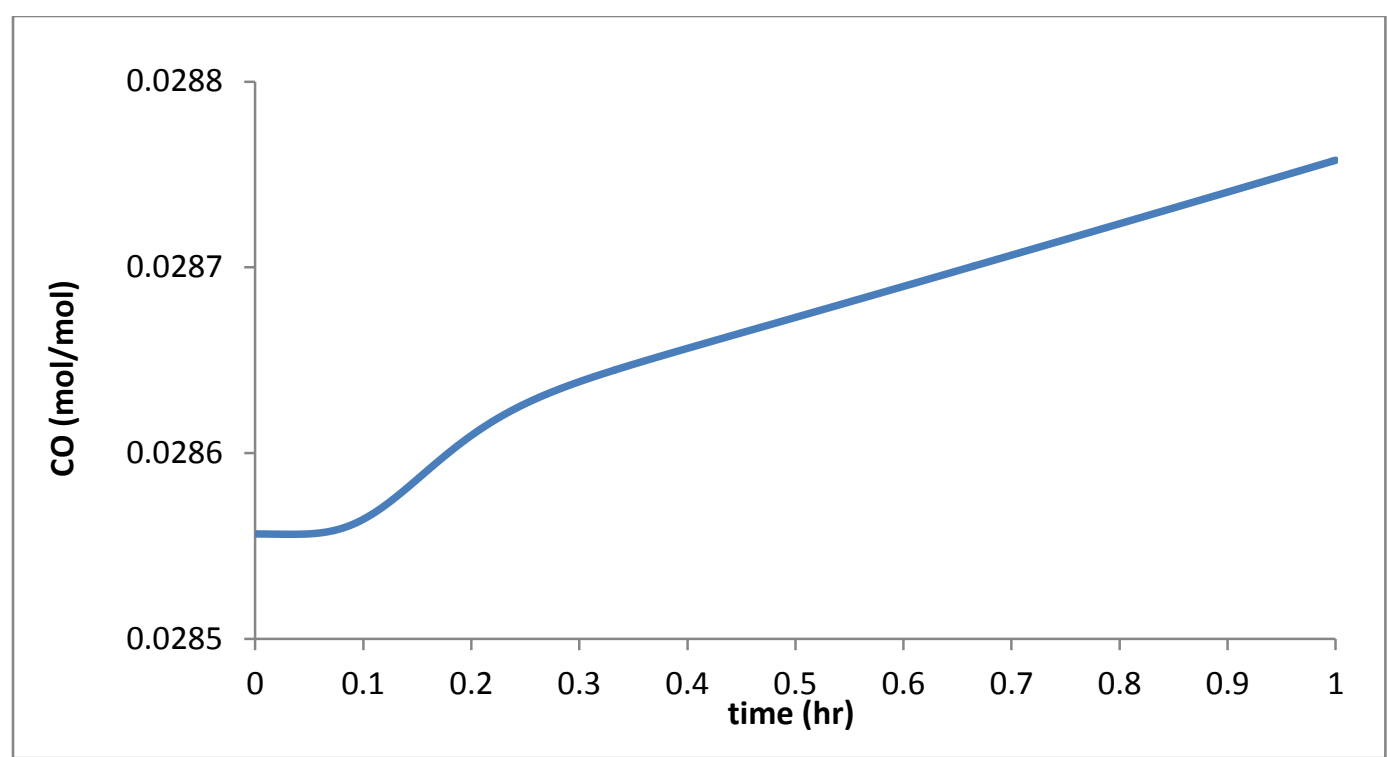

Figure 7.11: $\mathrm{CO}$ at end of $\mathrm{R} 2$ as a result of ramp decrease in porosity

From Figure 7.11, it can be seen that the effect of the fault in R1 has very small impact on the overall conversion at the outlet of R2.

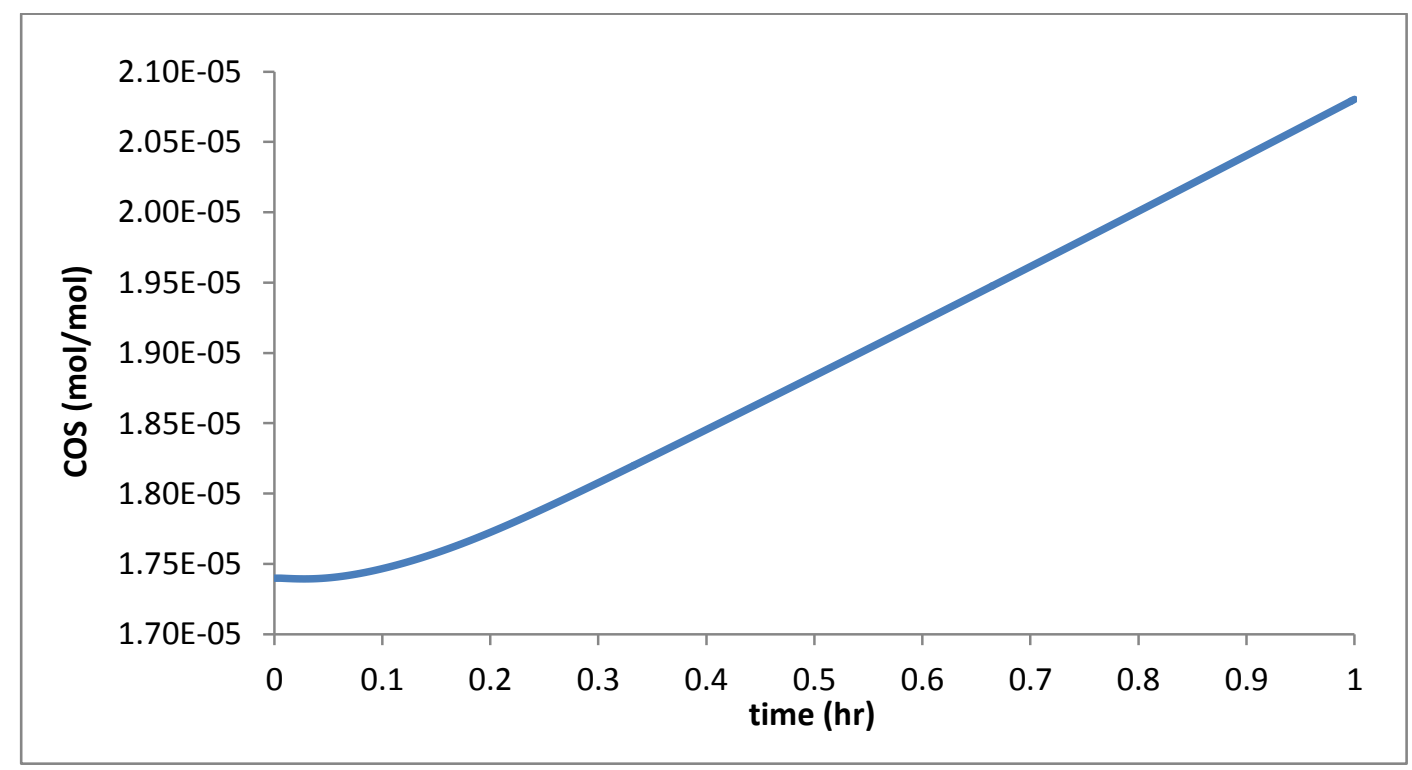

Figure 7.12: COS at end of $\mathrm{R} 2$ as a result of ramp decrease in porosity

As shown in Figure 7.12, COS seems to show stronger response than $\mathrm{CO}$ but the overall change in COS conversion is still negligible. 


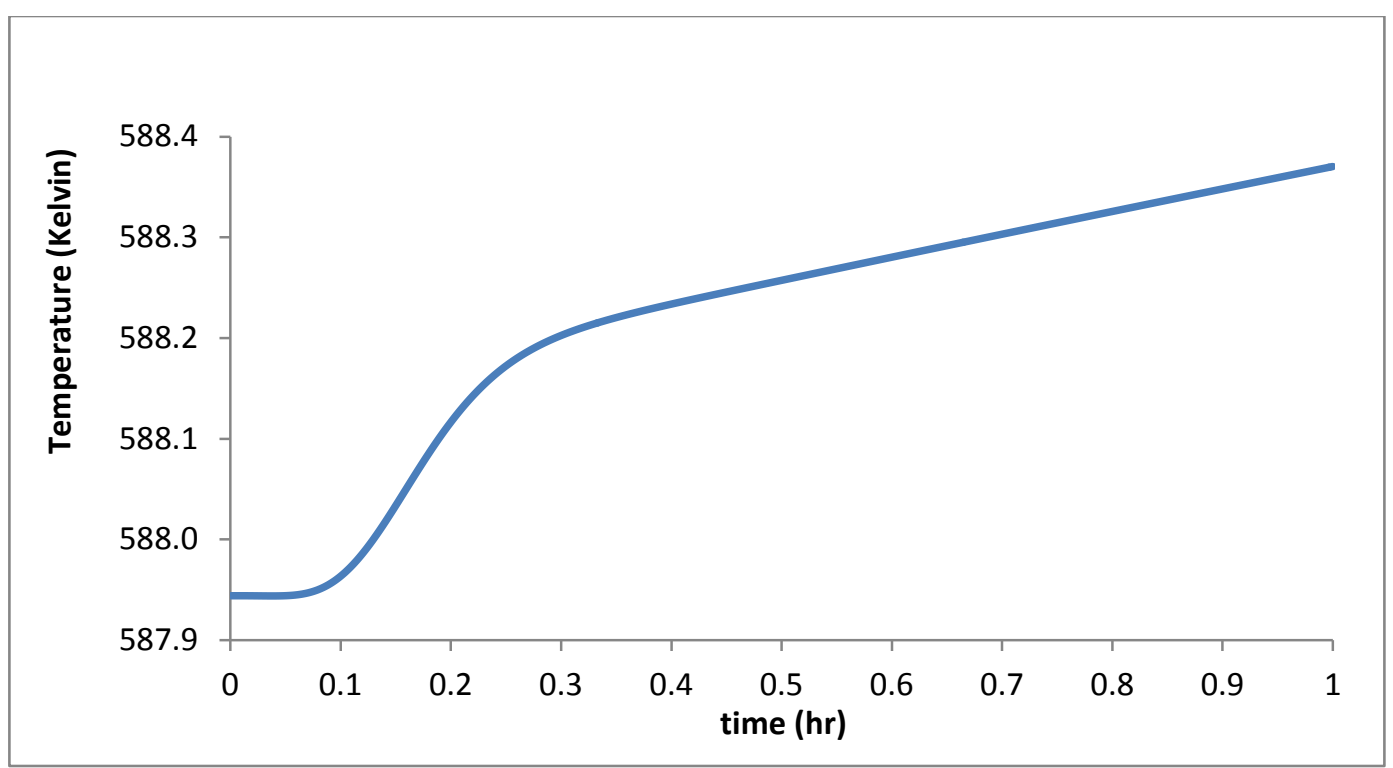

Figure 7.13: Temperature response at the exit of $\mathrm{R} 2$ as a result of ramp decrease in porosity

Due to the increase in inlet $\mathrm{CO}$ composition, higher extent of reaction takes place in the R2. As the WGS reaction is exothermic, the temperature at the exit of the reactor increases as shown in Figure 7.13.

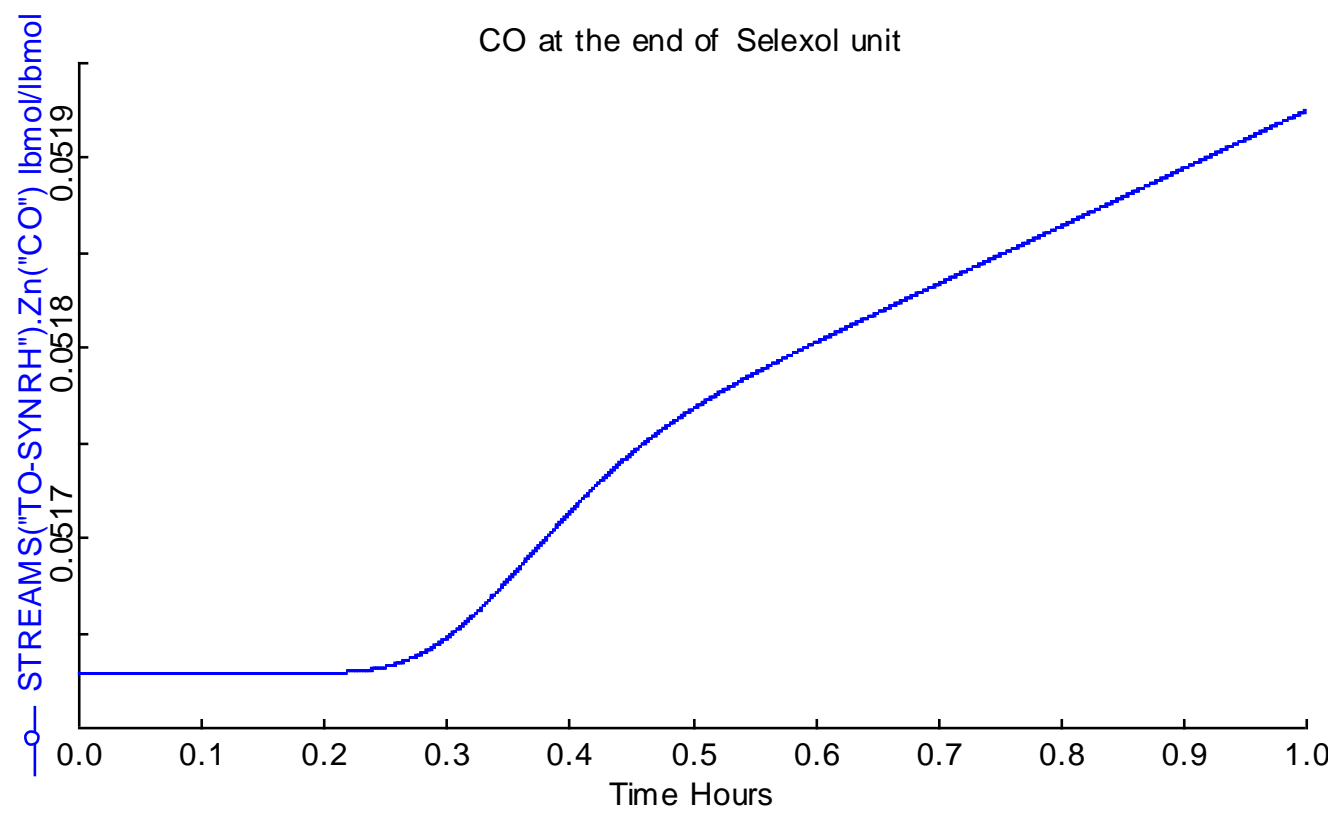

Figure 7.14: CO response at the exit of the Selexol unit as a result of ramp decrease in porosity

The increase in $\mathrm{CO}$ concentration as a result of the ramp decrease in porosity is also reflected in the clean syngas stream exiting the SELEXOL unit that is being sent to the combined cycle 
power plant. Figure 7.14, taken directly from Aspen Plus Dynamics, is a plot that shows CO mole fraction increases due to this fault. However, it takes around $12-13$ minute for this effect to be seen. The changes in the mole fractions of other components are very small to be detected clearly by a measuring device. 


\subsubsection{Sensor Placement Results}

\subsubsection{System-Level: Gasification Island}

The system-level sensor placement algorithms are implemented on the gasification island and the results are presented in the Table 7.11. From the case studies in previous sections we have learned that the optimal results are obtained from the combination algorithm (FES \& MR). Here, since the magnitude of the faults considered in the gasification island is low (maximum 10\% change as a fault), therefore, intuitively low level MR threshold would be useful. This can be verified by the results in Table 7.10. The FES \& MR algorithm with low MR threshold level has the lowest number of unresolvable faults and sensor network cost. The results of SDG and FES individual algorithm are shown for comparison. The sensor network for FES \& MR with low MR threshold level is shown in Table 7.11. Note that almost all of the sensors picked for resolution are temperature sensors except for a concentration sensor on the first stage sour WGS reactor. Since the temperature sensors are the least expensive sensors in this study, system-level fault resolution has been achieved with a significantly cost effective sensor network. Of the 703 pair of fault sets, only 25 pairs of faults could not be resolved. This implies that more than $96 \%$ of the faults considered in the system can be resolved by a cost effective network of sensors.

The study shows that even sensors at the SWGSR can be useful in identifying faults simulated in the SELEXOL side of the gasification island.

Table 7.10: System-level sensor placement results of gasification island

\begin{tabular}{ccccc}
\hline Algorithm(s) & $\begin{array}{c}\text { MR } \\
\text { threshold } \\
\text { level }\end{array}$ & Number of sensors & $\begin{array}{c}\text { Sensor network } \\
\text { cost }\end{array}$ & Number of unresolvable fault sets \\
\hline SDG & & 112 & 21.8 & 26 \\
FES & 13 & 30.9 & 25 \\
& Low & 11 & 11.7 & 25 \\
MR & Medium & 11 & 21.7 & 26 \\
& High & 12 & 21.8 & 26 \\
& Low & 11 & 10.9 & 25 \\
\multirow{2}{*}{ FES \& MR } & Medium & 13 & 30.9 & 25 \\
& High & 13 & 30.9 & 25 \\
\hline
\end{tabular}


Table 7.11: List of sensors for FES \& MR algorithm with low MR threshold level

\begin{tabular}{crc}
\hline $\begin{array}{c}\text { Sensor } \\
\text { number }\end{array}$ & Sensor location & $\begin{array}{c}\text { Sensor } \\
\text { Type }\end{array}$ \\
\hline 1 & Outlet stream of water from $2^{\text {nd }} \mathrm{HE}$ before $\mathrm{H} 2 \mathrm{~S}$ absorber & Temperature \\
2 & Cooled syngas stream from outlet of $3^{\text {rd }} \mathrm{HE}$ before $\mathrm{H} 2 \mathrm{~S}$ absorber & Temperature \\
3 & Syngas stream inlet $\mathrm{T}$ to HE right before $\mathrm{H} 2 \mathrm{~S}$ absorber & Temperature \\
4 & SELEXOL stream after H2SSTRBT fed to H2S concentrator & Temperature \\
5 & Bottoms stream of H2S concentrator & Temperature \\
6 & Lean SELEXOL stream after being heater by H2SSTRBT from SELEXOL stripper & Temperature \\
7 & Stream at the outlet of $\mathrm{HE}$ where $\mathrm{F}_{22-24}$ is simulated & Temperature \\
8 & $9^{\text {th }}$ tray in $\mathrm{CO}_{2}$ absorber & Temperature \\
9 & CO sensor at $75 \%$ length of R1 & Composition \\
10 & Temperature sensor at grid $65 \%$ length of R1 & Temperature \\
\hline
\end{tabular}

\subsubsection{Component-Level: First-Stage Sour Water Gas Shift Reactor}

The distributed sensor placement is performed on the first stage sour WGS reactor. A modified extended Kalman filter (EKF) is used here. Each fault is assumed as a state and is augmented with other states in the system and is estimated by the EKF. White Gaussian noise with known mean and variance is added to each state. The faults considered in the component-level are $\mathrm{F}_{25}$, $\mathrm{F}_{26}$ and $\mathrm{F}_{27}$ for catalyst porosity reduction; and $\mathrm{F}_{31}$ and $\mathrm{F}_{32}$ for catalyst activity reduction. Since the faults are modeled as a reduction in the catalyst activity each fault state is assumed to be associated with a process noise. Figure 7.15 shows the progress of the GA for different faults. Using only 30 sensors optimally placed on the reactor, more than $60 \%$ accuracy of measuring all the states (201 sensors on $\mathrm{CO}, \mathrm{H}_{2} \mathrm{O}, \mathrm{CO}_{2}, \mathrm{H}_{2}, \mathrm{COS}$ and $\mathrm{H}_{2} \mathrm{~S}$ mole fractions, temperature and pressure states) has been achieved. Table 7.12 shows the optimal solution, sensor type and location, for each fault. The numbers in Table 7.12 show the grid-point number out of 25 total available grid-points of the corresponding sensor type at which measurement must be made. The grid-point numbers represent the location of the sensors on the reactor and the variable names represent the sensor type. Using the optimal sensor placements, each corresponding fault state is estimated and plotted in Figure 7.16. This figure shows that the fault severities are estimated with reasonable accuracy. 


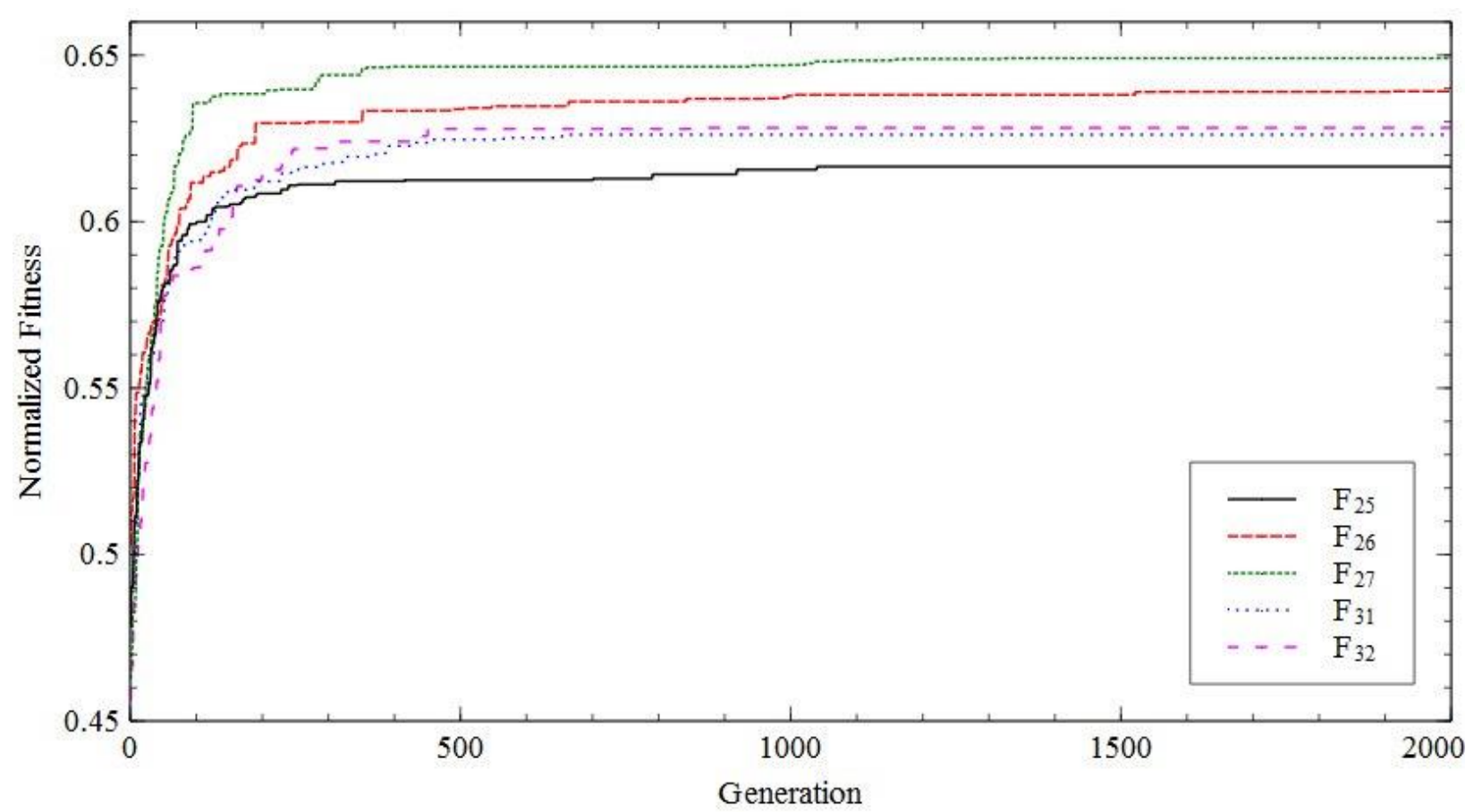

Figure 7.15: GA progress for different faults

Table 7.12: Optimal location and type of sensors for different faults

\begin{tabular}{cccccc}
\hline Sensor Type & \multicolumn{5}{c}{ Sensor Location } \\
\hline & $\mathrm{F}_{25}$ & $\mathrm{~F}_{26}$ & $\mathrm{~F}_{27}$ & $\mathrm{~F}_{31}$ & $\mathrm{~F}_{32}$ \\
\cline { 2 - 6 } $\mathrm{T}_{\mathrm{g}}$ & $21,22,24$ & 17,21 & 15,17 & 10,21 & $13,14,24$ \\
$\mathrm{P}$ & & & & \\
$\mathrm{P}_{\mathrm{in}}$ & & & & \\
& & & & & \\
$\mathrm{CO}$ & $13,16,21$, & $11,14,21$, & $13,20,21,22,23$, & $20,21,15,19$, & $19,20,21$, \\
& $22,23,24,25$ & $22,23,24,25$ & 24,25 & 24,25 & $22,23,24,25$ \\
$\mathrm{COS}$ & 25 & $23,24,25$ & $22,23,24,25$ & $22,24,25$ & $19,20,21,22$, \\
$\mathrm{H}_{2} \mathrm{O}$ & 13 & 14 & 13 & 12 & $23,24,25$ \\
& $2,7,8,9$, & $4,6,8,10$, & $5,14,15,17$, & $3,5,14,17$, & $3,13,14$, \\
$\mathrm{CO}_{2}$ & $10,11,12,14,1$ & $11,12,13$, & $19,20,21$, & $18,22,23$, & $16,21,23,24,2$ \\
& $5,23,24,25$ & $15,16,22$, & $22,23,24,25$ & 24,25 & 5 \\
$\mathrm{H}_{2}$ & $10,11,12$, & $23,24,25$ & $14,15,17$, & $14,15,16$, & $13,14,15,16,1$ \\
$\mathrm{H}_{2} \mathrm{~S}$ & $14,15,16$ & $12,13,15,16$ & 19,21 & 17,18 & 7 \\
\hline
\end{tabular}




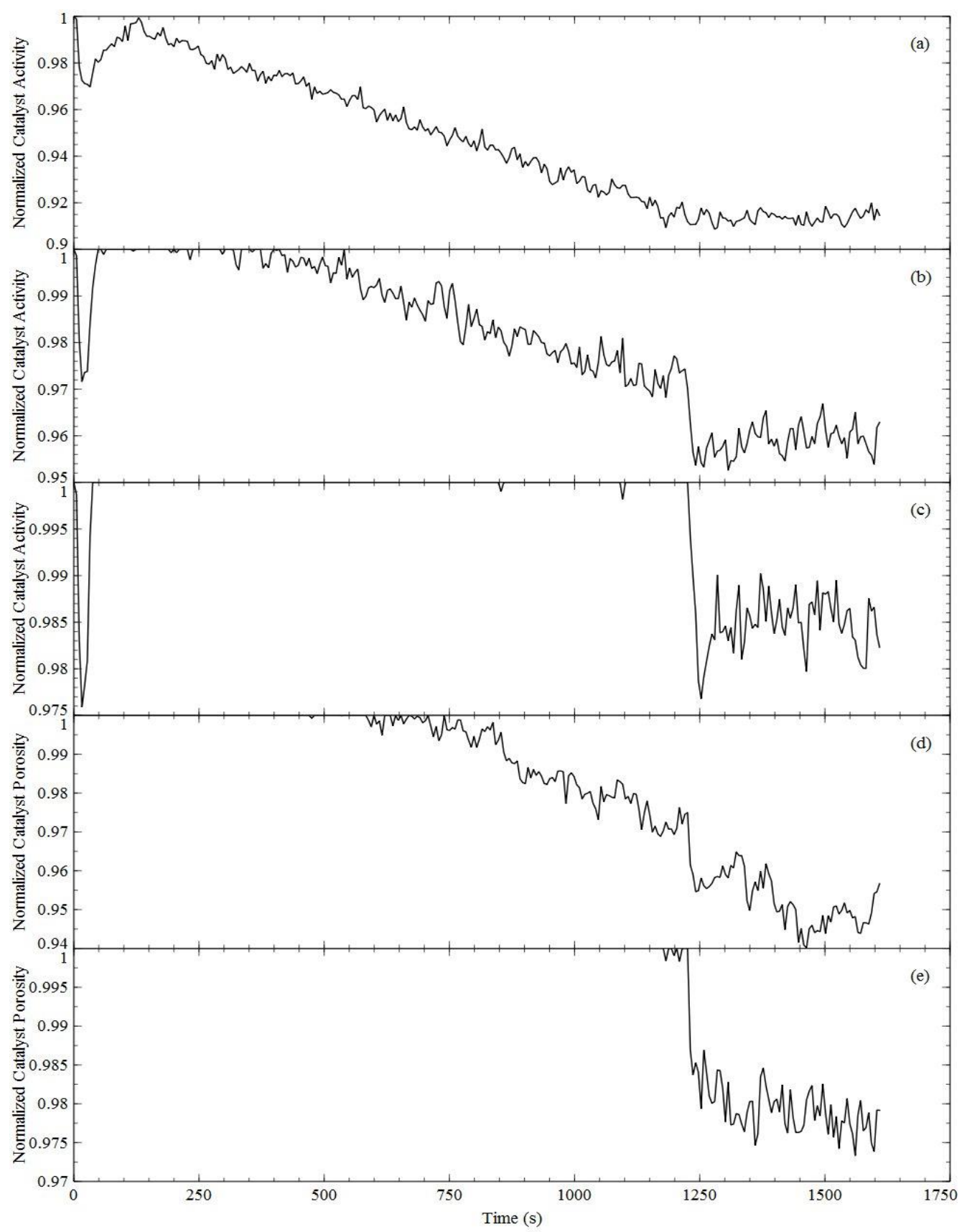

Figure 7.16: Fault severity estimation using optimal solution for fault (a) $\mathrm{F}_{25}$ (b) $\mathrm{F}_{26}$ (c) $\mathrm{F}_{27}$ (d) $\mathrm{F}_{31}(\mathrm{e}) \mathrm{F}_{32}$

These sensors along with all the sensors obtained by distributed sensor placement are combined to make up the total sensors for the component monitoring and fault severity estimation. While some sensor type and locations are similar for each fault, there are 66 unique sensors when the sensor sets are combined. These 66 sensors are combined with the two sensors placed on the reactor in system-level sensor placement. Also, since usually the states at the outlet of the reactor 
are measured for control and product specification purposes, 8 more sensors are placed at the reactor outlet to measure the mole fractions, temperature and pressure. This results in a set of 72 unique sensors. Therefore, in the integrated sensor placement which is the combination of the sensors obtained in the system- and component-level sensor placement, a network of 72 sensors is used for state monitoring and fault severity estimation.

Table 7.13: Comparison of normalized fitness values for GA solution and final sensor network

\begin{tabular}{ccc}
\hline Fault & GA solution normalized fitness & Final sensor network normalized fitness \\
\hline $\mathrm{F}_{25}$ & 0.6168 & 0.7561 \\
$\mathrm{~F}_{26}$ & 0.6391 & 0.7716 \\
$\mathrm{~F}_{27}$ & 0.6491 & 0.7751 \\
$\mathrm{~F}_{31}$ & 0.6261 & 0.7800 \\
$\mathrm{~F}_{32}$ & 0.6282 & 0.7749 \\
\hline
\end{tabular}

Table 7.13 compares the normalized fitness values using the optimal sensor placements and the final integrated sensor network. Using the final sensor network, there is an improvement in the estimation of quality of all the states in the reactor and not only the fault states.

\subsection{Conclusion}

The system-level sensor placement resulted in a set of sensors that help in fault resolution for most of the faults although some faults remain unresolved. For each fault in the component-level of sour WGS reactor, type, location, and number of sensors are obtained by solving the optimization problem where each set can be used to estimate the severity of the corresponding fault with desired accuracy.

A few sensors obtained by system-level sensor placement are placed on the sour WGS reactor. Using the additional sensors, it was found that the estimation of the variables in the reactor improved. Overall, the reactor can be monitored efficiently using the sensor network. 


\section{CONCLUSIONS}

In this work, detailed distributed PDAE based models of two key equipment items in the IGCC power plant have been developed for component level sensor placement studies. In the HSCSP model of the entrained-flow gasifier, the shrinking core model is applied in the initial region of the gasifier while a novel shrinking particle model is developed for the later region. This model is more physically realistic than the traditional SCM, yet yields similar results. The SPM is developed by integrating a continuous model with a particle model. Carbon conversion and gasifier exit conditions obtained from the HSCSP model compare well with the industrial data. The model provides information about the particle density of char particles, fraction of slag droplets that are attached to the char particles, and fraction of slag droplets that are detached but exist in the bulk. In addition, the sizes of char particles and attached slag droplets can be tracked along the gasifier. It is also observed that even though the slag detachment is a discontinuous phenomenon, the profile of the volume fraction of detached slag remains reasonably smooth. The number of slag droplets separated per unit volume per unit time is found to increase considerably as the detachment diameter decreases. This information is very valuable for calculating the slag deposition rate on the gasifier wall. The model has been further enhanced into a dynamic slagging gasifier model with models of slag transport, deposition, and slag layer. The model also considers both char particle and slag droplet impaction as mechanisms by which slag gets added to the slag flow layer. It was found that the char particle impaction is the dominant mechanism in the initial section of the gasifier while the slag droplet deposition dominates in the later section. The model is modified to incorporate the PSD of slag droplets from literature, however it is seen that the effect on slag layer thickness does not change greatly. The enhanced gasifier model is able to capture the effect of the dynamics and operation conditions of the gasifier on the slag layer to identify key features in this type of gasifier. The $\mathrm{O}_{2} /$ coal ratio is an important input to the unit and has a strong effect on the slag layer thickness. Although an increase in the ratio can reduce the slag layer thickness and could increase the carbon conversion, it may be at the cost of accelerated refractory degradation, especially at the hot spot. The silica ratio is another parameter that affects the slag layer thickness strongly and the model is able to quantify the effect on the 
silica ratio on the slag layer thickness. The results for coal switch are also presented to show the effect on the outlet composition and conversion, gasifier wall temperature and slag layer thickness and viscosity. Knowledge of the effects of coal switch can greatly help design operating procedures to ensure safe and continuous operation of the gasifier.

A refractory degradation model has also been developed. It has been assumed that the change in the refractory layer thickness and its properties do not affect the gasifier operating conditions. A 2-D model of the wall is developed and is used to identify the location where the slag penetration depth is the maximum. This location is then used for modeling the refractory degradation. Two methods are considered in this work. A compressive spalling model is developed and used to calculate the time and thickness of the first spall at the selected location. This model shows that an increase in temperature from $1800 \mathrm{~K}$ to $1850 \mathrm{~K}$ effectively reduces the time for the first spall by half. In addition, a thermoelastic model of the refractory layers has been developed by considering the layers to be concentric cylinders. The model is able to capture the radial, hoop and axial stress profile in the radial direction at the selected location where the temperature and slag penetration rate is the highest.

A 1-D dynamic model of a sour water gas shift reactor has also been developed as part of the component level modeling. Equations are written for a catalyst based reactor system and the equipment is modeled as a plug flow reactor. Reaction kinetics for the WGS reaction have been estimated from the reconciled data. A sulfur tolerant catalyst is considered that is able to convert COS conversion to $\mathrm{H}_{2} \mathrm{~S}$. A single stage SWGSR is first modeled and the effect of change in inlet conditions and catalyst deactivation is simulated. The model is then extended to a two-stage SWGSR system with an inter stage cooler and scaled to the industrial scale for integration in the IGCC plant. Several faults including change in the catalyst activity, porosity and surface area are simulated and the changes in the $\mathrm{CO}, \mathrm{COS}$ and temperature response at the outlet of the reactor are studied.

For system-level sensor placement, the process models of the SELEXOL plant and the CC power plant have been modified to simulate faults. Since it is intractable to record all the variables in the simulation, a selection criterion is applied and a reduced set of variables are recorded. SDG, FES, MR and a combination of FES and MR are used as sensor placement algorithms. Three levels of MR are considered-low, medium and high. 
In the SELEXOL unit, 14 faults are simulated. It is found that while the sensor network identified using all the SP algorithms are able to resolve all faults, the sensor network obtained using the combined MR and the FES-MR algorithms is the most cost effective. In the CC fault simulation, 21 faults are simulated. Faults at three levels of severity are simulated to check if it is possible for the sensor network to distinguish between them. Although not all these faults are resolvable, it is observed that the designed sensor network is able to distinguish among very similar faults. Overall, the designed sensor networks are able to identify and distinguish between most faults.

For the two-tier sensor placement, an integrated cross-platform simulation is set up to couple the two-stage SWGSR system model developed in MATLAB with the SELEXOL model developed in Aspen Plus Dynamics. The two models are synchronized and the faults at the component and system level are simulated. Faults at three severity levels are simulated generating a total of 41 faults. Sensor placement at both the component and system levels is performed to obtain the sensor network for the gasification island. At the system-level in the combined model, two sensors located at the SWGSR are used along with the sensors in the SELEXOL plant to resolve about $96 \%$ of the fault sets in the SELEXOL unit. The final sensor network on the equipment unit developed using the two-tier SP strategy is able to provide a better estimate of the faults in comparison to considering only the component level SP. This shows that the two-tier SP approach is able to use the interaction of the process at the component and system level to generate a more efficient sensor network. 


\section{RECOMMENDATIONS AND FUTURE WORK}

The sensor placement algorithms developed in this report can easily be used for different processes. This requires the availability of a mathematical model of the processes and an appropriate state estimation technique for the unit-level sensor placement. When the plant-wide process model is available, fault simulations and system-level sensor placement can be performed. A major difficulty would be the implementation of system-level sensor placement on very large processes. For large processes, a future research on network decomposition can help in reducing the complexity of the problem and make the system-level sensor placement more tractable.

Through the use of process model and a state estimation technique, the component-level sensor placement is performed. The main issue in the component-level sensor placement approach is the complexity of detail process models resulting in increased computation time for solving the state estimation and, underlying optimization problems. Future research in this area can focus on order reduction methods for process models. Method of characteristics and In Situ Adaptive Tabulation (ISAT) are two methods which have been implemented on the sour WGS reactor as part of this project resulting in reduction in computational cost. CFD-based models for generating multi-zonal ROMs could also be a viable method of reducing the computational complexity while maintaining the level of detail in the model.

Due to the complexity of the gasifier, multiple time and spatial scales in this equipment item and the discontinuities associated with the spalling and degradation process, the gasifier model cannot be directly used in the component level sensor placement framework. While the dynamics of the gasifier manifests in seconds or minutes, the slag penetration and refractory degradation mechanisms take place over the period of months or years. Running both models together, for a simulation time of months, is impractical for the purposes of sensor placement using the approach similar to the sour water gas shift reactor. Phenomena occurring at different time scales can be separated to make them amenable for sensor placement algorithms. 
For reduction in the simulation time for the gasifier model, a linear model of the gasifier can be developed by linearizing the model around the operating conditions of interest and integrating it with the refractory degradation model. Candidate locations for sensor placement will not only be limited to various locations in the axial direction, but should also include the depth into the refractory at which the sensor can be embedded.

In addition to the compressive spalling mechanism considered in this work, tensile spalling mechanisms due to slag penetration should be considered as well. Tensile spalling can occur when chrome $\left(\mathrm{Cr}^{3+}\right)$ from the high chrome refractory migrates out of the refractory matrix. This leads to the formation of cracks in the refractory brick and a decrease in the strength of the refractory, eventually leading to spalling. Refractory degradation can take place in the absence of slag as well through mechanisms such as creep, thermal fatigue and thermal shock. These mechanisms are known to take place at high temperature and high stress conditions. Creep is the slow deformation of a material at elevated conditions which result in the loss of material strength, eventually weakening the material. Thermal fatigue occurs due to the cyclic variation in temperature. The properties of the material begin to deteriorate over the course of several cycles and this change is irreversible. The level of degradation due to thermal fatigue depends on the number of cycles, the temperature, its magnitude of the fluctuation of temperature and the frequency. When there is a rapid change in temperature, the sudden increase in the temperature gradient in the refractory brick can lead to a large build-up of stress.

The current refractory degradation model can be expanded to account for other degradation mechanisms. Another recommendation would be to consider the combined effect of these mechanisms so as to capture how they interact and affect the total time to failure. It is likely that some degradation mechanisms may be dominant in certain sections of the gasifier. This framework for refractory degradation would be helpful to identify the key degradation mechanisms in various sections of the gasifier, and thus help in the selection of the appropriate type of sensors for fault detection. Eventually such studies can be used to develop mitigation and control strategies for improving the refractory life.

For the sensor placement, the reliability of sensors can be included into the algorithm to obtain a more robust sensor network. Some sensors are more reliable than other sensors, and the SP algorithm may select the final sensor network based on its cost as well as overall reliability. The 
selection of redundant sensors based on these criteria can also improve the reliability of the sensor network. 


\section{REFERENCES}

Adams, T.A.; Barton, P. I. A dynamic two-dimensional heterogeneous model for water gas shift reactors. Int. J. Hydrogen Energy. 2009, 34 (21), 8877-8891.

Ahmadi, G. Ash \& pulverized coal deposition in combustors \& gasifiers. Quarterly Technical Progress Report, Pittsburgh Energy Technology Center, USA, 1996.

Aldridge, C.L. Shift process for new catalyst. Google Patents: 1974.

Amadeo, N.E.; Laborde, M. A. Hydrogen production from the low-temperature water-gas shift reaction: Kinetics and simulation of the industrial reactor. Int. J. Hydrogen Energy. 1995, 20 (12), 949-956.

Andreev, A.A.; Kafedjiysky, V. J.; Edreva-Kardjieva, R. M. Active forms for water-gas shift reaction on Ni Mo-sulfide catalysts. Appl. Catal. A Gen. 1999, 179 (1-2), 223-228.

Anthony, B.D.; Howard, B. J. Coal devolatilization and hydrogasification. AIChE Journal. 1976, $22(4), 625-656$.

Arastoopour, H.; Gidaspow, D. Vertical pneumatic conveying using four hydrodynamic models. Ind. Eng. Chem. Fund. 1979, 18(2),123-130.

Aspen Custom Modeler, v7.2, AspenTech, Massachusetts, United States.

Aspen Plus Dynamics, v7.2, AspenTech, Massachusetts, United States.

Aspen Technology-Aspen Physical Property System, v7.1, AspenTech, Massachusetts, United States.

Bartholomew, C.H. Mechanisms of catalyst deactivation. Appl. Catal. A Gen. 2001, 212 (1-2), 17-60.

Bearth, C. Mathematical modeling of entrained flow coal gasification. PhD. Thesis, Department of chemical engineering, University of Newcastle, Australia, 1996.

Bell, N.H.; Edgar, T.F. Modelling of a fixed-bed water-gas shift reactor: 1. Steady-state model verification. J. Process Control. 1991, 1(1), 22-31.

Bell, N.H.; Edgar, T.F. Modelling of a fixed-bed water-gas shift reactor: 2. Variable catalyst activity effects and dynamic testing. J. Process Control. 1991, 1(2), 59-67. 
Bennett, J.P.; Kwong, K.S. Failure mechanisms in high chrome oxide gasifier refractories. Metallurgical and Materials Transactions A: Physical Metallurgy and Materials Science, 2011, 42(4), 888-904.

Benyon, P.J. Computational modeling of entrained flow slagging gasifiers. PhD thesis, University of Sydney. 2002. Australia.

Berispek, V. Studies of an alkali impregnated cobalt-molybdate catalyst for the water-gas shift and the methanation reactions. PhD Thesis. Virginia Polytechnic Institute and State University, 1975.

Bhattacharyya D.; Turton R.; Zitney S. Load-following control of an IGCC plant with $\mathrm{CO}_{2}$ capture. Proc. of the 28th Annual International Pittsburgh Coal Conference, Pittsburgh, PA, 2011.

Bhattacharyya D..; Turton R.; Zitney S. Control system design for maintaining $\mathrm{CO}_{2}$ capture in IGCC power plants while load-following. Proc. of the 29th Annual International Pittsburg Coal conference, Pittsburgh, PA, 2012.

Bhattacharyya, D.; Turton, R.; Zitney, S. E. Steady-State Simulation and Optimization of an Integrated Gasification Combined Cycle Power Plant with CO2 Capture. Ind. Eng. Chem. Res. 2010, 50 (3), 1674-1690.

Bhushan, M.; Rengaswamy, R. Design of sensor location based on various fault diagnostic observability and reliability criteria. Comput. Chem. Eng. 2000, 24 (2-7), 735-741.

Bird, R.B.; Stewart, E.S.; Lightfoot, E.N. Transport Phenomenon. 2nd ed., WSE Wiley, 2002.

Bockelie M.J.; Denison M.K.; Chen Z.; Linjewile T.; Senior C.L.; Sarofim A.F. CFD modeling for entrained flow gasifiers in Vision 21 systems. Proceedings of the 19th Annual International Pittsburgh Coal Conference. September 24-26, Pittsburgh, PA, 2002.

Buhre B.J.P.; Hinkley J.T.; Gupta R.P.; Nelson P.F.; Wall T.F. Fine ash formation during combustion of pulverised coal-coal property impacts. Fuel. 2006, 18(2), 185-193.

Chen, C.; Horio, M.; Kojima, T. Numerical simulation of entrained flow gasifiers. 1: modeling of coal gasification in an entrained flow gasifier. Chem. Eng. Sci. 2000, 55, 3861-3874.

Chen, E. Behavior of refractory linings for slagging gasifier. Thesis. Massachusetts Institute of Technology, 1984.

Chen, E.; Buyukozturk, O. Methodology for thermomechanical analysis of brittle systems. Amer. Ceram. Soc. Bull. 1985, 64 (7), pp. 982-988. 
Chen, W.H.; Lin, M.R.; Jiang, T.L.; Chen, M.H. Modeling and simulation of hydrogen generation from high-temperature and low-temperature water gas shift reactions. Int. J. Hydrogen Energy. 2008, 33 (22), 6644-6656.

Cho, S.H.; Yoo, J.I.; Turley, A.T.; Miller, C.A.; Linak, W.P.; Wendt, J.O.L.; Huggins, F.E.; Gilmour, M.I. Relationships between composition and pulmonary toxicity of prototype particles from coal combustion and pyrolysis. Proc. Combust. Inst. 2009, 32, 2717-2725.

Choi, Y.C.; Li, X.Y.; Park, T.J.; Kim, J.H.; Lee, J.G. Numerical study on the coal gasification characteristics in an entrained flow coal gasifier. Fuel. 2001, 80, 2193-2201.

Clayton S.J.; Siegel G.J.; Wimer J.G. Gasification technologies, gasification markets and technologies - present and future, an industry perspective. US Department of Energy. 2002; DOE/FE-0447.

De la Osa, A.R.; De Lucas, A.; Valverde, J.L.; Romero, A.; Monteagudo, I.; Sánchez, P. Performance of a sulfur-resistant commercial WGS catalyst employing industrial coalderived syngas feed. Int. J. Hydrogen Energy. 2011, 36 (1), 44-51.

Ding, O. L.; Chan, S. H. Water-gas shift reaction A 2-D modeling approach. Int. J. Hydrogen Energy. 2008, 33 (16), 4325-4336.

Doraiswamy, L. K.; Sharma, M. M. Heterogeneous Reactions: Analysis, Examples, and Reactor Design. 1: Gas-solid and solid-solid reactions, John Wiley \& Sons, New York, 1984.

Ergun, S. Fluid flow through packed columns. Chem. Eng. Prog. 1952, 48.

Fiedorow, R.; Léauté, R.; Lana, I. G. D. A study of the kinetics and mechanism of COS hydrolysis over alumina. J. Catal. 1984, 85 (2), 339-348.

Francesconi, J.A.; Mussati, M.C.; Aguirre, P. A. Analysis of design variables for water-gas-shift reactors by model-based optimization. J. Power Sources. 2007, 173 (1), 467-477.

Friedrichs, G.; Wagner, H. Direct Measurements of the reaction $\mathrm{NH} 2+\mathrm{H} 2 \rightarrow \mathrm{NH} 3+\mathrm{H}$ at temperatures from 1360 to 2130 K. Zeitschrift für Physikalische Chemie. 2000, 214(8), 1151160.

Fulton C.H. Principles of Metallurgy. Norwood, MA: Plimption Press, 1910.

George, Z.M. Kinetics of cobalt-molybdate-catalyzed reactions of SO2 with $\mathrm{H} 2 \mathrm{~S}$ and COS and the hydrolysis of COS. J. Catal. 1974, 32 (2), 261-271. 
Giunta, P.; Amadeo, N.; Laborde, M. Simulation of a low temperature water gas shift reactor using the heterogeneous model/application to a PEM fuel cell. J. Power Sources. 2006, 156 (2), 489-496.

Govind, R.; Shah, J. Modeling and simulation of an entrained flow gasifier. AIChE Journal. 1984, 30 (1), 79-92.

Guha, A. Transport and Deposition of Particles in turbulent and laminar Flow. Annu. Rev. Fluid Mech. 2008, 40, 311-41.

Gunawardana, P.V.D.S.; Lee, H.C.; Kim, D. H. Performance of copper-ceria catalysts for water gas shift reaction in medium temperature range. Int. J. Hydrogen Energy. 2009, 34 (3), $1336-1341$.

Lijie, G.; Jianxin, K. A Hybrid Process Monitoring and Fault Diagnosis Approach for Chemical Plants. Int'l J. of Chem. Eng. 2015. doi:10.1155/2015/864782

Haaland, S.E. Simple and Explicit Formulas for the Friction Factor in Turbulent Flow. J. Fluids Eng. ASME. 1983, 105(1), 89-90.

Hakkarainen, R.; Salmi, T.; Keiski, R.L. Water-gas shift reaction on a cobalt-molybdenum oxide catalyst. Appl. Catal. A Gen. 1993, 99 (2), 195-215.

He, M.Y.; Evans, A.G.; Huchinson, J.W. Effects of Morphology on the decohesion of Compressed Thin Films. Phys. Status Solidi A. 1998, 166, 19-35.

Helble J.J.; Srinivasachar, S.; Boni A.A. Factors influencing the transformation of minerals during pulverized coal combustion. Progress in Energy and Combustion Sciences. 1990, 16, 267-279.

Hetnarski, R.B.; Eslami, M.R. Thermal Stresses - Advanced Theory and Application. $1^{\text {st }}$ ed., Springer, 2010.

Hla, S.S.; Duffy, G.J.; Morpeth, L.D.; Cousins, A.; Roberts, D.G.; Edwards, J.H. Investigation into the performance of a $\mathrm{Co}-\mathrm{Mo}$ based sour shift catalyst using simulated coal-derived syngases. Int. J. Hydrogen Energy. 2011, 36 (11), 6638-6645.

Hoggan, P.E.; Aboulayt, A.; Pieplu, A.; Nortier, P.; Lavalley, J.C. Mechanism of COS hydrolysis on alumina. J. Catal. 1994, 149 (2), 300-306.

Hou, P.; Meeker, D.; Wise, H. Kinetic studies with a sulfur tolerant water gas shift catalyst. $J$. Catal. 1983, 80 (2), 280-285. 
Jakobsen, H.A. Chemical Reactor Modeling: Multiphase Reactive Flows; Springer: New York, 2008.

Johnson, K.I.; Williford, R.E.; Matyas, K.; Pilli, S.P.; Sundaram, S.K.; Korolev, V.N. Modeling Slag Penetration and Refractory Degradation Using the Finite Element Method. Proceedings Int'l Pittsburgh Coal Conference, 2008.

Jones, W.P.; Lindstedt, R.P. Global reaction schemes for hydrocarbon combustion. Combustion and Flame. 1988, 73, 233-249.

Kang, S.G.; Kerstein, A.R.; Helble, J.J.; Sarofim, A.F. Simulation of residual ash formation during pulverized coal combustion bimodal ash particle size distribution. Aerosol Science and Technology. 1990, 13, 401-412.

Karan, K.; Mehrotra, K.A.; Behie, A.L. A high-temperature and modeling study of homogeneous gas-phase COS reactions applied to Claus plants. Chem. Eng. Sci. 1999, 54, 2999-3006.

Kasule, J.S.; Turton, R.; Bhattacharyya, D.; Zitney, S.E. One-dimensional dynamic modeling of a single-stage downward-firing entrained-flow coal gasifier. Energy \& Fuels. 2014, 28(8), 4949-4957.

Kasule, J.S.; Turton, R.; Bhattacharyya, D.; Zitney, S.E. Mathematical modeling of a singlestage, downward-firing, entrained-flow gasifier. Ind. Eng. Chem. Res. 2012, 51(18), 64296440.

Kasule, J.S. Mathematical Modeling and Simulation of a One-Dimensional Transient Entrainedflow GEE/Texaco Coal Gasifier. PhD Thesis, Department of Chemical Engineering, West Virginia University, USA, 2012.

Kobayashi, H.; Howard, J.B.; Sarofim, A.F. Coal devolatilization at high temperatures. Proc. $16^{\text {th }}$ International Symposium on Combustion, 1976.

Kumar, M.; Ghoniem A.F. Multiphysics simulations of entrained flow gasification. Part 1: Validating non-reacting flow solver and particle turbulent dispersion model. Energy \& Fuels. 2012, 46, 451-463.

Kwong, K.; Petty, A.; Bennett, J.; Krabbe, R.; Thomas, H. Wear mechanisms of chromia refractories in slagging gasifiers; Int'l J. Applied Ceram. Tech. 2007, 4(6), 503-513.

Lee, H.H.; Lee, J.C.; Joo, Y.J.; Lee, C.H. Dynamic modeling of Shell entrained flow gasifier in an integrated gasification combined cycle process. Appl. Energy. 2014, 131, 421-440. 
Lei, C.; Yong, S.Z.; Ghoniem, A.F. Modeling the slag behavior in three dimensional CFD simulation of a vertically-oriented oxy-coal combustor. Fuel Process. Technol. 2013, 112, 106-117.

Levenspiel, O. Chemical Reaction Engineering. 2nd ed., John Wiley \& Sons, New York, 1972.

Levent, M. Water-gas shift reaction over porous catalyst: Temperature and reactant concentration distribution. Int. J. Hydrogen Energy. 2001, 26 (6), 551-558.

Li, S.; Whitty, K.J. Physical phenomena of char-slag transition in pulverized coal gasification. Fuel Process. Tech. 2012, 95, 127-136.

Li, S.; Wu, Y.; Kevil, W. Ash deposition behavior during char-slag transition under simulated gasification conditions. Energy \& Fuels. 2010, 24(3), 1868-1876.

Li, Y.; Wang, R.; Chang, L. Study of reactions over sulfide catalysts in $\mathrm{CO}-\mathrm{CO} 2-\mathrm{H} 2-\mathrm{H} 2 \mathrm{O}$ system. Catal. Today. 1999, 51 (1), 25-38.

Liang, Q.; Guo, X.; Dai Z.; Liu, H; Gong X. An investigation on the heat transfer behavior and slag deposition of membrane wall in pilot scale entrained flow gasifier. Fuel. 2012, 102, 491498.

Lim, S.; Bae, J.; Kim, K. Study of activity and effectiveness factor of noble metal catalysts for water-gas shift reaction. Int. J. Hydrogen Energy. 2009, 34 (2), 870-876.

Liu, B.; Garcia, H.E.; Baxter, L.L. A simplified phase equilibrium algorithm used to predict ash/slag behaviors in slagging gasifiers/combustors. 25th Annual International Pittsburgh Coal Conference; Pittsburgh, PA; United States; 2008.

Liu, S.G.; Rezaei, H.R.; Lucas, A.J.; Harris, J.D.; Wall, F. T. Modelling of a pressurized entrained flow coal gasifier; the effect of reaction kinetics and char structure. Fuel. 2000, 1767-1779.

Loehden D.; Walsh P.M.; Sayre A.N.; Beer J.M.; Sarofim A.F. Generation and deposition of fly ash in the combustion of pulverized coal. J. Institute of Energy. 1989, 451, 119-127.

Ma J. Methods and Systems for Fault Diagnosis in Nuclear Power Plants. PhD Thesis, School of Graduate and Postdoctoral Studies, The University of Western Ontario London, Ontario, Canada, 2015

Maloney D.; Monazam K.; Casleton K.H.; Shaddix C.R. Evaluation of char combustion models: measurement and analysis of variability in char particle size and density. Proceedings of the Combustion Institute. 2005, 30, 2197-2204. 
MATLAB and Statistics Toolbox Release 2012b, The MathWorks, Inc., Natick, Massachusetts, United States.

McCollor, D.P.; Zygarlicke, C.J.; Allan, S.E.; Benson, SA. Ash Deposit Initiation in a Simulated Fouling Regime. Energy \& Fuels. 1993, 7(6), 761-767.

McGee, T.D. High Temperature Creep of Refractory Bricks"; Final Report ORNL/SUB-8007940/01, Oakridge National Laboratory, 1991.

Mehta A.S.; Sahajwalla, V. Influence of temperature on the wettability at the slag/carbon interface during pulverised coal injection in a blast furnace. Scandinavian Journal of Metallurgy. 2001, 30, 370-378.

Mobed, P.; Maddala, J.; Rengaswamy, R.; Bhattacharyya, D.; Turton R. Data Reconciliation and Dynamic Modeling of a Sour Water Gas Shift Reactor. Ind. Eng. Chem. Res. 2014, 53, 19855-19865.

Moe, J. Design of water-gas shift reactors. Chem. Eng. Prog. 1962, 58 (3).

Monaghan R.F.D.; Ghoniem A.F. A dynamic reduced order model for simulating entrained flow gasifiers: Part I: Model development and description. Fuel. 2012, 91(1), 61-80.

Monaghan, R.F.D.; Ghoniem, A.F. A dynamic reduced order model for simulating entrained flow gasifiers. Part II: Model validation and sensitivity analysis. Fuel. 2012, 94, 280-297.

Monaghan, R.F.D.; Ghoniem, A.F. Simulation of a commercial-scale entrained flow gasifier using reduced order model. Energy \& Fuels. 2011, 26, 1089-1106.

Niessen W.R. Combustion and incineration processes, 3rd ed. CRC Press, Basel, NY, 2010.

Niksa, S.; Kerstein, A.R. FLASHCHAIN theory for rapid coal devolatilization kinetics, 1.Formulation. Energy and Fuels. 1991, 5, 647-665.

Nowok, J.W. Viscosity and phase-transformation in coal ash slags near and below the temperature of critical viscosity. Energy \& Fuels. 1994, 8(6), 1324-1336.

Pednekar, P.; Bhattacharyya, D.; Kasule, J.S.; Turton, R.; Rengaswamy, R. Development of a hybrid shrinking core shrinking particle model for entrained flow gasifiers. AIChE J. 2016, 62(3), 659-669.

Pednekar, P.; Bhattacharyya, D.; Kasule, J.S.; Turton, R.; Rengaswamy, R., Dynamic Model of a Slagging Entrained-Flow Gasifier Including Models of Slag Transport, Deposition, and Slag Layer, Ind. Eng. Chem. Res. 2016 55, 1, 279-292. 
Peters, N. Premixed burning in diffusion flames-The flame zone model of Libby and Economos. Int. J. Heat Mass Transfer, 1979, 22, 691-703.

Quann R.J.; Sarofim A.F. A scanning electron microscopy study of the transformations of organically bound metals during lignite combustion. Fuel.1986, 65, 40-46.

Rao, R.M.; Bhattacharyya, D.; Rengaswamy, R.; Choudhury, R.S. A two-dimensional steady state model including the effect of liquid water for a PEM fuel cell cathode. Journal of Power Sources. 2007, 173, 375-393.

Rase, H.F.; Holmes, J.R. Chemical reactor design for process plants. Vol. 2. Wiley, New York, 1977.

Raiman, O. Order of magnitude reasoning. Artif. Intell. 1991, 51 (1-3), 11-38.

Rees, O.W. Composition of the ash of Illinois coals, Circular 365, Illinois State Geological Survey, 1964.

Robinson, P.J.; Luyben, W.L. Simple Dynamic Gasifier that runs in Aspen Dynamics. Ind. Eng. Chem. Res. 2008, 47, 7784-7792.

Rowe, P.N.; Henwood, G.A. Drag forces in hydraulic model of fluidized bed. Trans. Chem. Eng. 1961, 39, 43-54.

Satterfield, C.N. Mass transfer in heterogeneous catalysis. RE Krieger Publishing Company, Massachusetts, 1981.

Seames, W.S. An initial study of the fine fragmentation fly ash mode generated during pulverized coal combustion. Fuel Processing Tech. 2003, 81, 109-125.

Seggiani, M. Modelling and simulation of time varying slag flow in a Prenflo entrained-flow gasifier. Fuel.1998, 77(14), 1611-1621.

Shah, J.; Govind, R. Modeling and simulation of an entrained flow coal gasifier. AIChE J. 1984, 30(1), 79-92.

Shimizu, T.; Tominaga, H. A model of char capture by molten slag surface under hightemperature gasification conditions. Fuel. 2006, 85, 170-178.

Siegel, R.; Howell, R.J. Thermal Radiation Heat Transfer, McGraw-Hill Book Company, New York, 1981.

SIMULINK, Version 2012b, The MathWorks, Inc., Natick, Massachusetts, United States.

Singh, C.P.P.; Saraf, D.N. Simulation of High-Temperature Water-Gas Shift Reactors. Ind. Eng. Chem. Proc. Des. Dev. 1977, 16 (3), 313-319. 
Srinivasachar, S.; Senior C.L.; Helble J.J.; Moore J.W. A fundamental approach to the prediction of coal ash deposit formation in combustion systems. Symp. Int'l. Combust. 1992, 24(1), 1179-1187.

Stalder, A.F.; Melchior, T.; Muller, M.; Sage, D.; Blu, T.; Unser, M. Low-Bond Axisymmetric drop shape analysis for surface tension and contact angle measurements of sessile drops. Colloids and surfaces A: Physicochemical and Engineering Aspects. 2010, 364, 72-81.

Streeter, R.C.; Diehl, E.K.; Schobert, H.H. Flow properties of low rank coal-ash slags Implications for slagging flow gasification. Fuel. 1985, 64, 1611-1617.

Sun, J.; DesJardins, J.; Buglass, J.; Liu, K. Noble metal water gas shift catalysis: Kinetics study and reactor design. Int. J. Hydrogen Energy. 2005, 30 (11), 1259-1264.

Sundaram, S.K.; Johnson, K.I.; Matyas, J. Williford, R.E.; Pilli, S.P.; Korolev, V.N. An Integrated Approach to Coal Gasifier Testing, Modeling, and Process Optimization. Fuel. 2009, 23 (10), 4748-4754.

Svoronos, P.D.N.; Bruno, T.J. Carbonyl Sulfide: A Review of Its Chemistry and Properties. Ind. Eng. Chem. Res. 2002, 41 (22), 5321-5336.

Syamlal, M.; Bissett, L.A. METC gasifier advanced simulation (MGAS) model, Technical Note, NITS report No. DOE/METC-92/4108 (DE92001111), 1992.

Tampa Electric POLK Power Station Integrated Gasification Combined Cycle Project, Final Technical report; Tampa Electric Company; Tampa, FL., 2002. http://www.netl.doe.gov/technologies/coalpower/cctc/cctdp/biblography

Thoenes, Jr, D.; Kramers, H. Mass transfer from spheres in various regular packings to a flowing fluid. Chem. Eng. Sci. 1958, 8 (3-4), 271-283.

Ubhayakar, K.S.; Stickler, B.D.; Gannon, E. R. Modeling of entrained-bed pulverized coal gasifiers. Fuel. 1977, 56, 281-291.

Vamvuka, D.; Woodburn, E.T; Senior, P.R. Modeling of an entrained flow coal gasifier, 1. Development of the model and general predictions. Fuel. 1995, 74, 1452-1460.

Vamvuka, D.; Woodburn, E.T; Senior, P.R. Modeling of an entrained flow coal gasifier, 2. Effect of operating conditions on reactor performance. Fuel. 1995, 74, 1461-1465.

Vaysman, V.; Lu, Y. Quality guidelines for energy system studies. Detailed coal specifications. DOE/NETL-401/012111, 2012. 
Wanatabe, H.; Otaka, M. Numerical simulation of coal gasification in entrained flow gasifier, Fuel. 2006, 85, 1935-1943.

Wang P.; Massoudi M. Slag behavior in Gasifiers. Part I: Influence of coal properties and gasification conditions. Energies. 2013, 6, 784-806.

Wang, X.; Zhao, D.; Jiang, L.; Yang, W. The deposition and burning characteristics during slagging co-firing coal and wood: modeling and numerical simulation. Combust. Sci. Technol. 2009, 181, 710-728.

Wang, X.H.; Zhao, D.Q.; He, L.B.; Jiang, L.Q.; He, Q.; Chen, Y. Modeling of coal-fired slagging combustor: Development of a slag submodel. Combust. Flame. 2007, 149, 249-260.

Wen, C.Y.; Chaung, T. Z. Entrained coal gasification modeling. Ind. Eng. Chem. Process Des. Dev. 1979, 18 (4), 684-695.

Wen, C.Y.; Chen, H.; Onozaki, M. C. User's manual for computer simulation and design of the moving-bed coal gasifier. DOE/MC/16474-1390, NITS/DE83009533. 1982.

Wen. C.Y. Noncatalytic heterogeneous solid-fuel reaction models. Ind. Eng. Chem. 1968, 60 (9), 34-54.

Westbrook, K.C.; Dryer, L.F. Simplified reaction mechanisms for the oxidation of hydrocarbon fuels in flames. Combustion Science and Technology. 1981, 27, 31-43.

Williford, R.E.; Johnson, K.I.; Sundaram, S.K. Modelling of high-chromia refractory spalling in slagging coal gasifiers. Ceram. Intl. 2008, 34(8), 2085-2089.

Williford, R.E.; Johnson, K.I.; Sundaram, S.K.; Pilli, S. Effective Diffusivity and Spalling Models for Slagging Coal Gasifiers. J. American Ceram Soc. 2008, 91(12), 4016-4022.

Wood, N.B. A simple method for the calculation of turbulent deposition to smooth and rough surfaces. J. Aerosol Sci. 1981, 12, 275-290.

Woods, M.C.; Capicotto, P.; Haslbeck, J.L.; Kuehn, N.J.; Matuszewski, M.; Pinkerton, L.L.; Rutkowski, M.D.; Schoff, R.L.; Vaysman, V. Cost and performance baseline for fossil energy plants. Volume 1: Bituminous coal and natural gas to electricity final report. NETL/DOE- 2010/1397, 2007.

Wu, H.; Wall, T. The effect of Pressure on Ash formation during Pulverized Coal Combustion. Energy \& Fuels. 2000, 14, 745-750. 
Wu, H.; Wall, T.; Liu, G.; Bryant, G. Ash liberation from included minerals during combustion of pulverized coal: the relationship with char structure and burnout. Energy \& Fuels. 1999, 13, 1197-1202.

Wang, X.; Zhao, D.; Jiang, L.; Yang, W. The deposition and burning characteristics during slagging co-firing coal and wood: modeling and numerical simulation. Combust. Sci. Tech. 2009, 181, 710-728.

Wang, X.H.; Zhao, D.Q.; He, L.B.; Jiang, L.Q.; He, Q.; Chen, Y. Modeling of a coal-fired slagging combustor: Development of a slag submodel. Combust. Flame. 2007, 149, 249-260.

Yagi, S.; Kunii, D. Studies on the combustion of carbon particles in flames and fluidized beds. Proceedings of the 5th International Symposium on Combustion, Reinhold, New York, 1955, 231-244.

Yan, L.; Gupta, R.P.; Wall, T.F. A mathematical model of ash formation during pulverized coal combustion. Fuel. 2002, 81, 337-344.

Yang, Z.; Wang, Z.; Wu, Y.; Wang, J.; Lu, J.; Li, Z.; Ni, W. Dynamic model for an oxygenstaged slagging entrained flow gasifier. Energy \& Fuels. 2011, 25, 3646-3656.

Ye, I.; Ryu, C. Numerical modeling of slag flow and heat transfer on the wall of an entrained flow gasifier. Fuel. 2015, 150, 64-74.

Yong, S.Z.; Gazzino, M.; Ghoniem, A. Modeling the slag layer in solid fuel gasification and combustion - formulation and sensitivity analysis. Fuel. 2012, 92, 162-170.

Yong, S.Z.; Ghoniem, A.; Modeling the slag layer in solid fuel gasification and combustion two-way coupling with CFD. Fuel. 2012, 97, 457-466.

Yu, D.; Xu, M.H.; Liu, X.; Huang, J.; Li, G. Mechanisms of submicron and residual ash particle formation during pulverised coal combustion: A comprehensive review. Developments in Chemical Engineering and Mineral Processing. 2005, 13(3-4), 467-482.

Zhang, L; Characterization of Combustion-Derived individual fine particulates by computer controlled scanning electron microscopy. AIChE J. 2009, 55(11), 3005-3016. 


\section{APPENDIX A: SYSTEM-LEVEL SENSOR PLACEMENT}

In a process system, a change in one variable can cause change in one or more variables. These cause-effect (CE) relations among the variables are very useful for diagnosing faults in the system. The optimum number and locations of sensors for the diagnosis of a process can be identified through an algorithmic approach when process simulation is available.

The sensor placement problem at the system-level is solved using directed graph (DG), signed directed graph (SDG), fault evolution sequence (FES), and magnitude ratio (MR) based approaches in this work. The DG-based approach only considers whether the the impact of effects is measurable or not while the SDG-based approach also considers the sign, i.e. if the effect is positive (such as an increase in the variable which is the cause will result in an increase in the variable that is affected) or negative. Even if multiple variables can get affected with the same directionality when a variable is changed or due to occurrence of a fault, they may not be affected at the same time instant. The temporal evolution is exploited in the FES algorithm. Relative change between two variables due to a change in a variable or due to occurrence of a fault is valuable information which is exploited in the MR-based approach. In the FES- and MRbased approaches for SP, a set of virtual sensors are generated by pair-wise combination of the original list of candidate sensors. In the MR algorithm, these virtual sensors represent the magnitude ratio of the corresponding pairs. In the FES algorithm, these virtual sensors represent the sequence in which the corresponding pairs respond to the faults/variables.

After the faults are simulated, the temporal responses of the candidate sensor variables are collected. Only one fault is simulated at one time. All the faults are introduced at the same operating conditions. No disturbance is considered into the system. It should be noted that the sensor placement algorithms discussed below are developed by our collaborators from Texas Tech University. 


\section{DG and SDG Algorithms}

Let the total number of faults and candidate sensor locations is $M$ and $N$, respectively. Fault sets, which are sets of all the sensors that respond to a fault, are first generated. Due to large differences in the magnitude and direction of change in the process variables, it is important to consider a threshold value for each process variable while generating the fault sets. The extent of change in a particular process variable depends on its actual operating value, type, operating condition, noise and disturbances. The threshold values considered in this work for the various sensors are shown in Table A.1.

Table A.1: Threshold values for various sensors used in this work

\begin{tabular}{ll}
\hline Variable type & Threshold Values \\
\hline Temperature & $1^{\circ} \mathrm{F}$ \\
Level & 1 inch \\
Flow & $3 \%$ \\
Power & $3 \%$ \\
Concentration (mole fraction) & 0.01 \\
Pressure & $2 \mathrm{psi}$ \\
\hline
\end{tabular}

In the DG algorithm, if a variable changes beyond its threshold, a value of "1" is assigned, otherwise a value of " 0 " is assigned, i.e. $R_{D G} \in\{0,1\}$. This operation returns a row vector for each fault with the dimension of $1 \times N$ and performing this operation for $M$ faults will return matrix $A_{\mathrm{DG}}$ with the dimension of $M \times N$. In the SDG algorithm, a value of "1" is assigned if the variable changes beyond the upper limit and a value of "-1" is assigned if it changes beyond the lower limit. If the variable stays within its limits, a value of " 0 " is assigned. Note that the deviations are based on the incipient response of the variables. Therefore, $R_{S D G} \in\{-1,0,1\}$. Considering all the faults, the matrix $A_{\mathrm{SDG}}$ of dimension $M \times N$ is obtained. A constraint matrix is constructed by augmenting the observability $\left(A_{o b s}\right)$ and resolution matrices $\left(A_{\text {res }}\right) ; \quad A=\left[\begin{array}{l}A^{\text {obs }} \\ A^{\text {res }}\end{array}\right]$. For observability, $A^{o b s}=A^{D G}$. For fault resolution, symmetric difference sets are generated for each pair of faults by performing the following operation: $A_{i j}^{r e s}=A_{i}^{S D G} \cup A_{j}^{S D G}-A_{i}^{S D G} \cap A_{j}^{S D G}$. This 
operation results in matrix $A^{\text {res }}$ with the dimension ${ }^{M} C_{2} \times N$ (Bhushan and Rengaswamy, 2000; 2002; Raiman, 1991). It is possible that some of the faults may produce deviation in the same direction for the same set of variables. In that case, the corresponding rows in the observability matrix are the same. If $q$ rows are identical in the observability matrix, the resolution matrix will have ${ }^{q} C_{2}$ number of rows with zero elements. These faults cannot be resolved.

A binary integer programming problem for sensor placement is formulated for minimizing the sensor cost subjected to fault observability and resolution considering the candidate sensor variables as decision variables. A binary decision variable is assigned to each process variable; if the decision variable takes a value of "1" then a sensor is placed to measure that variable and a " 0 " value implies that the variable is not measured. The constraint matrix, A, in the optimization problem represents the coefficient matrix obtained by DG and SDG. Since for observability and resolution at least one sensor must be picked by the optimizer, the $b$ vector represents the constant vector of unity with $\left(M+{ }^{M} C_{2}\right)$ rows. Equation A.1 is solved as the optimization problem.

$$
\min \sum_{j}^{N} w_{j} x_{j}
$$

Subject to:

$$
A x^{T} \geq b
$$

where, $x_{j}$ denotes binary decision variable and $w_{j}$ denotes weight for the sensor $j=1, \ldots, N$. A and $\mathrm{B}$ are given by:

$$
A=\left[\begin{array}{cccccc}
1 & 0 & 0 & 1 & \cdots & 1 \\
0 & 0 & 1 & 1 & \cdots & 1 \\
\vdots & \vdots & \vdots & \vdots & \ddots & \vdots \\
0 & 1 & 0 & 1 & \cdots & 1 \\
& & & & & \\
& & & & &
\end{array}\right]_{\left(M+{ }^{M} C_{2}-{ }^{q} C_{2}\right) \times N} \quad b=\left[\begin{array}{c}
1 \\
1 \\
\vdots \\
1 \\
\\
\end{array}\right.
$$


The cost of the sensors is normalized by the cost of the flow sensor. The normalized values used in this work are shown in Table A.2.

Table A.2: Type and cost of each sensor used in all case studies

\begin{tabular}{ll}
\hline Sensor type & Cost \\
\hline Temperature sensor & 0.1 \\
Pressure sensor & 0.5 \\
Flow sensor & 1 \\
Level sensor & 1 \\
Concentration sensor & 10 \\
\hline
\end{tabular}

\section{Sensor Placement Using Fault Evolution Sequence (FES)}

A set of $M$ faults are modeled first simulated. The time at which a sensor exceeds the threshold value is also recorded in this algorithm. A total of ${ }^{N} C_{2}$ combinations are considered as the available pairs and a base sequence is considered for each pair as $\left\{S_{i}, S_{j}\right\}$ where $j>i$ and $S$ represents the corresponding sensor. The pairs used in this study are of the form $P_{i j}$, where $P_{i j}$ is the pseudo-sensor assigned to the sequence of sensors $S_{i}$ and $S_{j}$. This will result in the generation of $2(N-1)$ pseudo-sensors as pairs. If the sequence of any pair $\left(P_{i j}\right)$ is in the same sequence as the base pairs, a value of "1" is assigned to that pair variable, if the pair is in the opposite sequence to the base pairs, a value of " -1 " is assigned and for all other pair variables, a value of " 0 " is assigned. A threshold value for time is set as the minimum time obtained from the typical sampling time in the process industry. This threshold ensures that it is possible to detect the sequence of the response. If the difference in response time of the pair is greater than the time threshold then it will take the values as described, otherwise, a value of " 0 " is assigned. Therefore, $P \in\{-1,0,1\}$.

Figure A.1 shows the flowchart of the FES algorithm. Here, $G$ is the measured value, $t$ is the time elapsed from a reference time until the sensor magnitude (measured value) goes beyond the threshold value, $T_{i}$ and $T_{j}$ are the threshold values of the candidate variables, and $T_{t}$ is the time threshold. 


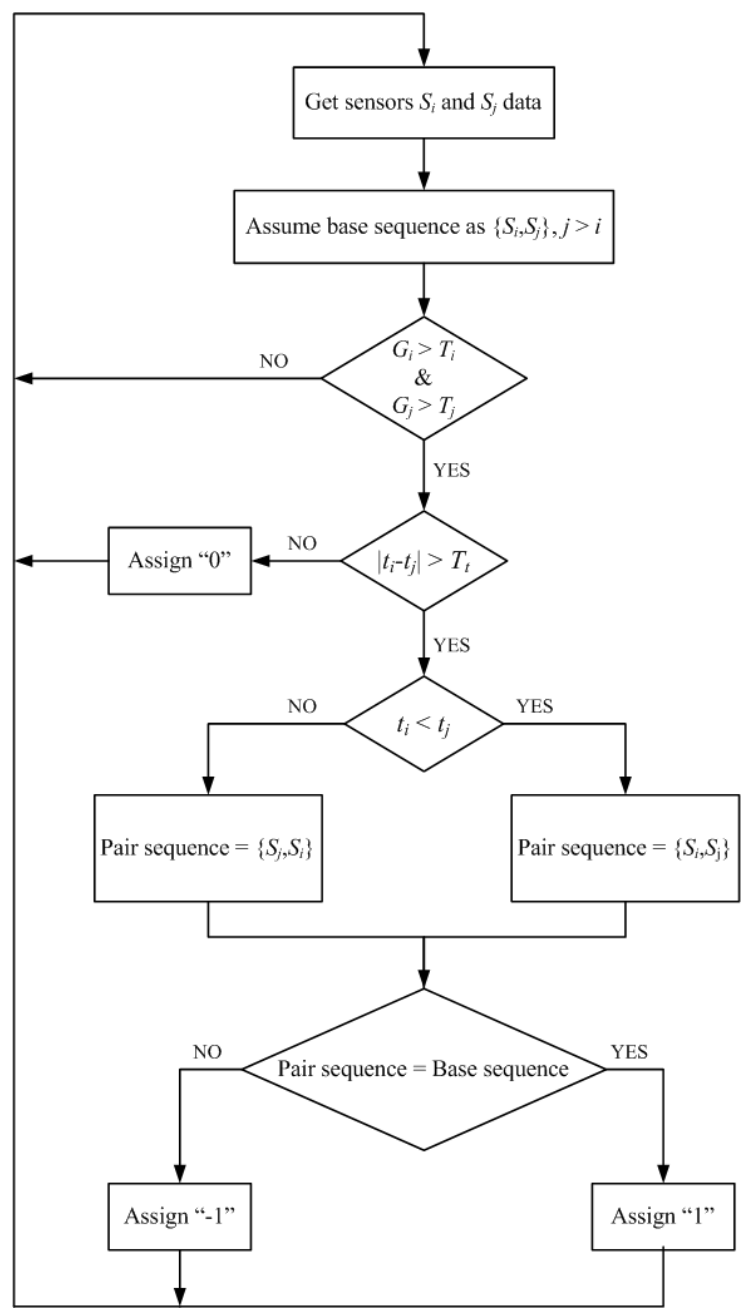

Figure A.1: Flowchart of FES algorithm in presence of numerical solution

The algorithm finally returns a matrix with dimension $M \times{ }^{N} C_{2}$ that is used to determine the optimal sensor network.

\section{Sensor Placement Using Magnitude Ratio}

In the MR based approach, the magnitude of the response of the sensors is important and is used to distinguish between the faults. The relation of interest here is whether $A>>B$ or $A \cong B \cong 1$, where, A and B represent the ratio of normalized magnitude of the sensors. To better understand the advantage of this idea, consider the example in Table A.3. Even if an SDG based algorithm cannot distinguish between $F_{1}$ and $F_{2}$, the ratio of the sensor measurements can provide additional information to distinguish between the two faults. Figure A.2 shows the ratio of $S_{1}$ to 
$S_{2}$ for each fault. Note that the magnitude of each sensor is normalized by its steady state value. The magnitude ratio for fault $F_{1}$ is much higher than that of $F_{2}$ 's. This indicates that the variable measured by sensor $S_{1}$ changes much more than that measured by sensor $S_{2}$, for fault $F_{1}$ but do not differ significantly for fault $F_{2}$.

Table A.3: SDG example to compare with MR based approach

\begin{tabular}{lll}
\hline Fault & \multicolumn{2}{c}{ Sensor } \\
\cline { 2 - 3 } & $S_{1}$ & $S_{2}$ \\
\hline$F_{1}$ & 1 & -1 \\
$F_{2}$ & 1 & -1 \\
\hline
\end{tabular}

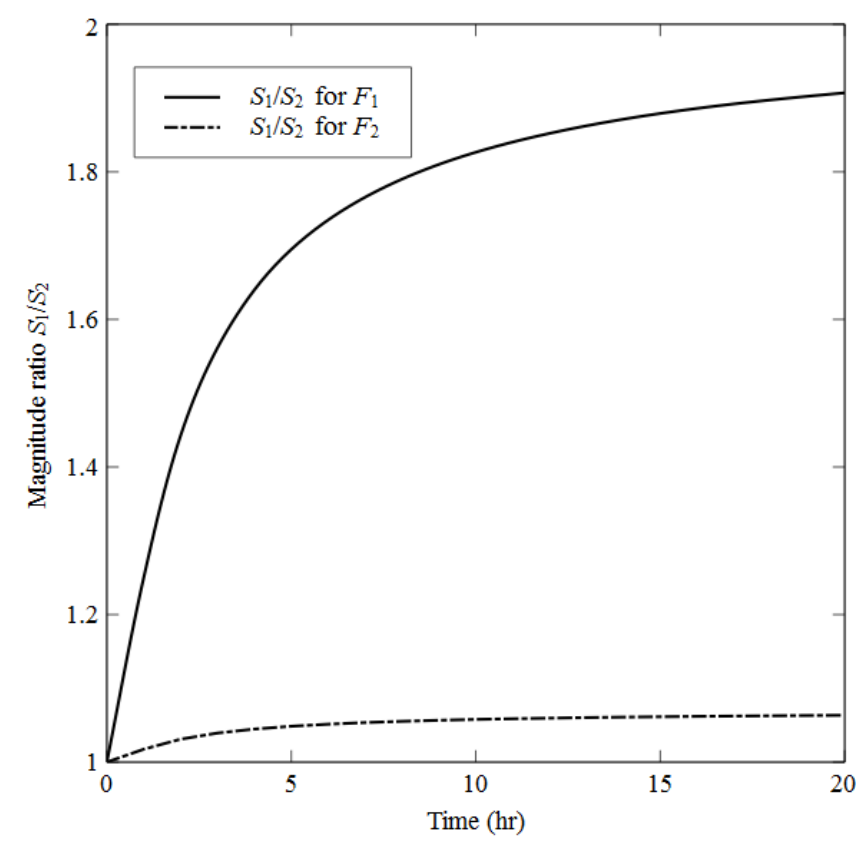

Figure A.2: Magnitude ratio for example in Table A.3

The magnitude ratio of a pair of sensors, $S_{i}$ and $S_{\mathrm{j}}$, is written as:

$$
r_{i j}=\frac{G_{i} / G_{i, S S}}{G_{j} / G_{j, S S}}
$$


where $G_{i}$ and $G_{j}$ are the magnitude of the sensors $S_{i}$ and $S_{j}$ and are normalized by their steadystate values $\mathrm{G}_{i, S S}$ and $\mathrm{G}_{j, S S}$, respectively. Note that both $S_{i}$ and $S_{j}$ must exceed their threshold value to be considered for further analysis. The magnitude ratio, $r_{i j}$, is initially set at a value of "1". After a fault is introduced to the system, $r_{i j}$ changes from its steady state ("1"), and can change in either direction. ${ }^{N} C_{2}$ pairs of sensors are generated and taken as pseudo sensors $P_{i j}$. If $r_{i j} \gg 1, P_{i j}$ is assigned a value of "1", if $r_{i j} \ll 1, P_{i j}$ is assigned a value of "-1", otherwise it is assigned a value of "0". Threshold values are identified to satisfy the inequalities and decided based on sensitivity analysis. $P \in\{-1,0,1\}$ is obtained for each of the $M$ faults and a matrix with dimension $M \times{ }^{N} C_{2}$ is generated similar to the FES algorithm. This matrix is considered as a constraint in the optimization problem for obtaining a sensor network.

Figure A.3 shows the flowchart of MR algorithm. In this flowchart, $T_{M R}$ is the MR threshold value.

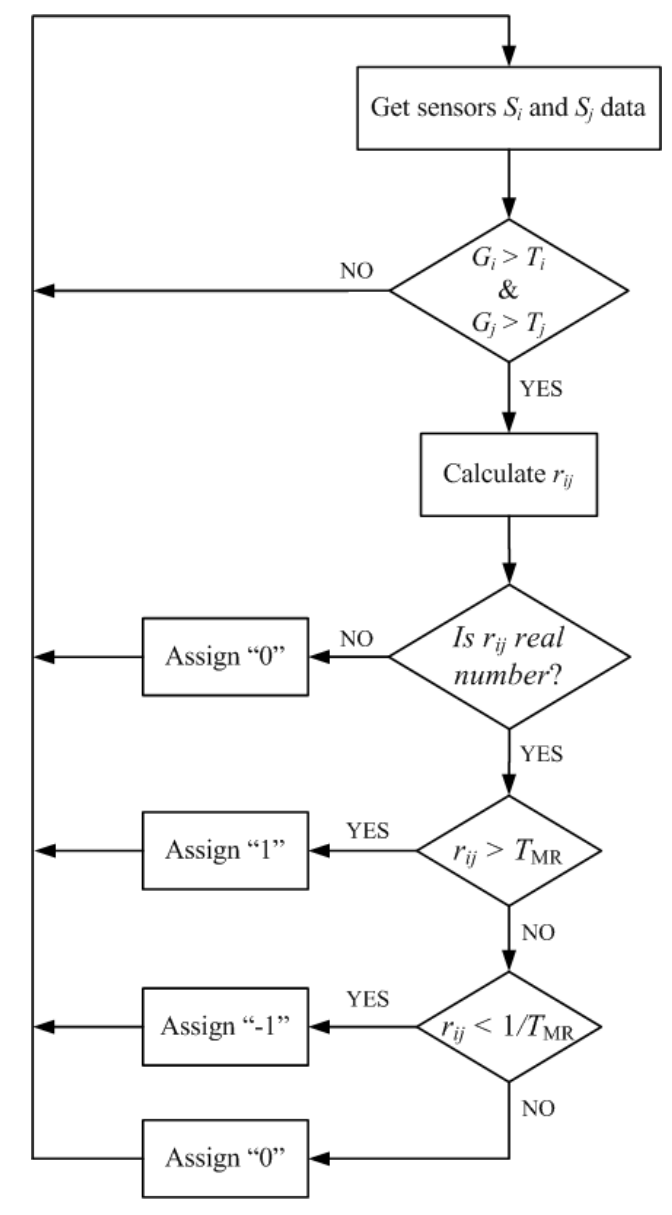

Figure A.3: Flowchart of MR algorithm 
MR algorithm is performed for three different levels of MR threshold values denoted as low, medium and high with values 1-1.2, 1.3-1.9 and greater than 1.9, respectively.

\section{Constraint matrix for FES and MR}

Similar to the resolvability problem of SDG, ${ }^{M} C_{2}$ pseudo-faults with corresponding symmetric differences of fault sets are constructed using the information provided by the $P$ matrix. This results in an ${ }^{M} C_{2} \times{ }^{N} C_{2}$ matrix containing the fault resolution information obtained from the FES or MR algorithm. Recall that the constraint matrix for DG/SDG approaches initially had $(M+$ ${ }^{M} C_{2}$ ) $\times N$ dimension with first $M$ rows representing observability and the following ${ }^{M} C_{2}$ rows representing resolution. To aid the resolution by FES or MR, the constraint matrix is augmented by ${ }^{N} C_{2}$ columns which consist of two blocks. An $M \times{ }^{N} C_{2}$ block of zeros is generated for observability, since FES and MR do not contribute to fault observability. In addition, an ${ }^{M} C_{2} \times$ ${ }^{N} C_{2}$ block is generated by taking symmetric difference on FES matrix (resulting in $A_{\text {FES }}$ matrix) or MR matrix (resulting in $A_{\mathrm{MR}}$ matrix). The new augmented constraint matrix is treated similar to the SDG where the rows that are same and the rows that contain only zeros are removed from the constraint matrix $A$ and vector $b$ before solving the optimization problem.

After construction of the new augmented constraint matrix, the optimization problem has $N+$ ${ }^{N} C_{2}$ decision variables, including the sensors and pseudo-sensors. However, to ensure consistency between the sensors and corresponding pseudo-sensors, the following constraints are added to the optimization problem (augmented constraint matrix) for each pseudo sensor:

$$
\begin{aligned}
& \left(1-x_{i}\right)+\left(1-x_{j}\right)+x_{i j} \geq 1 \\
& \left(1-x_{i j}\right)+x_{i} \geq 1 \\
& \left(1-x_{i j}\right)+x_{j} \geq 1
\end{aligned}
$$

where $x_{i j}$ is the pseudo-sensor corresponding to the sensors $x_{i}$ and $x_{j}$ in the decision variables. Eqn. A.3 ensures that $S_{i}$ and $S_{j}$ need to be selected if $S_{i j}$ is selected and vice versa. The three linear inequality constraints are added to the optimization problem constraints. Therefore, the constraint matrix and the constant vector have the dimension of $\left(3 \times{ }^{N} C_{2}\right) \times\left(N+{ }^{N} C_{2}\right)$ and $\left(3 \times{ }^{N} C_{2}\right) \times 1$, respectively. 
The optimization problem in Eqn. (A.1) for the FES and MR SP method can be summarized as:

$$
\min \sum_{j}^{N} w_{j} x_{j}
$$

Subject to:

$$
A x^{T} \geq b
$$

$x_{j}$ binary

where

A

$=\left[\begin{array}{cc}A^{\text {obs }} & 0_{M \times{ }^{N} C_{2}} \\ A^{\text {res }} & 0^{{ }^{M} C_{2} \times{ }^{N} C_{2}} \\ { }^{{ }^{M} C_{2} \times N} & A^{*} \\ A^{\text {cons }}\end{array}\right]_{\left(M+{ }^{M} C_{2}+3 \times{ }^{N} C_{2}-{ }^{q} C_{2}\right) \times\left(N+{ }^{N} C_{2}\right)}$

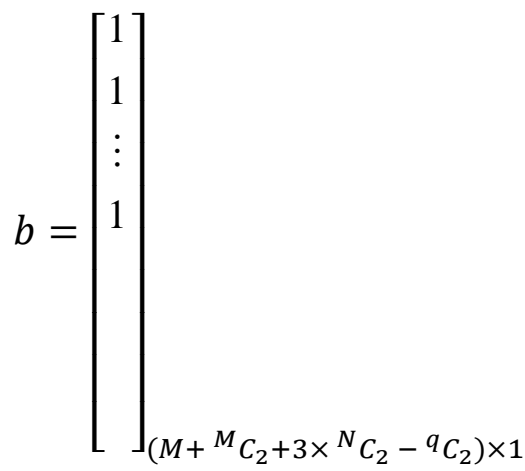

where $A^{*}=A^{F E S}$ for FES algorithm and $A^{*}=A^{M R}$ for MR algorithm. Note that a joint FES and MR algorithm can be obtained by $A^{*}=A^{F E S \& M R}=A^{F E S} \cup A^{M R}$.

\section{Solution Approach}

The sensor network design is posed as a BILP and can be solved by a number of commercially available optimization software. In this work, the optimization problem is formulated in MATLAB and the solution is obtained by the CPLEX optimization toolbox integrated in MATLAB. 


\section{APPENDIX B: COMPONENT-LEVEL SENSOR PLACEMENT}

Model based sensor placement for developing a sensor network at the component level can be done by considering that an optimal estimator has been placed to obtain estimates of the states in the unit in the presence of process and measurement noises. The optimization problem is solved by using a genetic algorithm (GA). The GA evaluates the "fitness" of the estimate for different combination of sensors and modifies the next sensor network provided to the estimator. The process is repeated for each fault simulation and the set of unique sensors for all fault simulation is used to obtain the final sensor network. A brief description of the component-level sensor placement is covered here.

\section{Extended Kalman Filter}

In order to estimate the states for a PDAE based model, the extended Kalman Filter (EKF) is used. The KF is used to estimate the states $x \in \mathrm{R}^{n}$ of a discrete-time controlled process governed by the linear stochastic difference equation:

$$
x_{k}=A x_{k-1}+B u_{k-1}+w_{k-1}
$$

with a measurement $\mathrm{z} \in \mathrm{R}^{n}$ given by

$$
z_{k}=H x_{k}+v_{k}
$$

Here, the matrix $A$ denotes the state transition matrix, the matrix $B$ denotes the input matrix $u \in$ $\mathrm{R}^{n}$ and matrix $H$ denotes the measurement matrix. $w_{k}$ and $v_{k}$ represent the process and measurement noises, respectively and are assumed to be normally distributed with zero mean as shown below:

$$
\begin{aligned}
& p(w) \sim N(0, Q) \\
& p(v) \sim N(0, R)
\end{aligned}
$$

$Q$ and $R$ are the process noise and measurement noise covariance matrix respectively. 
$\check{x}^{\prime}{ }_{k} \in \mathrm{R}^{n}$ is defined as the a priori state estimate at step $k$ calculated using the process model and $\breve{x}_{k} \in \mathrm{R}^{n}$ is the a posteriori state estimate at step $k$ calculated after the measurement information $z_{k}$ is obtained. The respective errors can be defined as:

$$
\begin{gathered}
e^{\prime}{ }_{k} \equiv x_{k}-\check{x}^{\prime}{ }_{k} \\
e_{k} \equiv x_{k}-\check{x}_{k}
\end{gathered}
$$

Based on this, the a priori estimate error covariance and the a posteriori estimate error covariance are defined as Eq. B.6-B.7.

$$
\begin{aligned}
& P^{\prime}{ }_{k}=E\left[e^{\prime}{ }_{k} e^{\prime}{ }_{k}{ }^{T}\right] \\
& P_{k}=E\left[e_{k} e_{k}{ }^{T}\right]
\end{aligned}
$$

The posteriori estimate is obtained using Eq. B.8.

$$
\check{x}_{k}=\check{x}_{k}^{\prime}+K\left(z_{k}-H \check{x}_{k}^{\prime}\right)
$$

$K$ is the Kalman gain.

$K$ can be calculated by:

$$
K_{k}=P^{\prime}{ }_{k} H^{T}\left(H P^{\prime}{ }_{k} H^{T}+R\right)^{-1}
$$

The estimation process essentially consists of two steps. The first step is the prediction step based on the process model to obtain an estimate of the states at time step $k$. The second step involves using sensor information (also subject to noise) to obtain a correction for the estimated states. The actual data used in the objective function is obtained by simulating the WGSR model.

The equations for the prediction and correction steps are shown in Table B.1 and B.2 respectively. 
Table B.1: Time update equations

$$
\begin{gathered}
\breve{x}_{k}^{\prime}=A \check{x}^{\prime}{ }_{k-1}+B u_{k-1} \\
P^{\prime}{ }_{k}=A P_{k-1} A^{T}+Q
\end{gathered}
$$

Table B.2: Measurement update equations

$K_{k}=P^{\prime}{ }_{k} H^{T}\left(H P^{\prime}{ }_{k} H^{T}+R\right)^{-1}$
$\check{x}_{k}=\check{x}^{\prime}{ }_{k}+K\left(z_{k}-H \check{x}^{\prime}{ }_{k}\right)$
$P^{\prime}{ }_{k}=A P_{k-1} A^{T}+Q$

The Kalman filter recursively conditions the current estimate on all the past measurements. The EKF works on the same predictor-corrector approach, but is a modification of the KF to consider non-linear systems. For non-linear systems, Eq. B.15 can be considered as the representation of the model.

$$
x_{k}=f\left(x_{k-1}, u_{k-1}, w_{k-1}\right)
$$

with the measurement model for $z$ given by:

$$
z_{k}=h\left(x_{k}, v_{k}\right)
$$

Approximate state and measurement can be calculated using B.17-18.

$$
\begin{aligned}
& \tilde{x}_{k}=f\left(\check{x}_{k-1}, u_{k-1}, 0\right) \\
& \check{z}_{k}=h\left(\tilde{x}_{k}, 0\right)
\end{aligned}
$$

To estimate a process with non-linear difference and measurement relationships, Eq. B.15-16 are first linearized to get B.19-20.

$$
\begin{aligned}
& x_{k} \approx \tilde{x}_{k}+A\left(x_{k-1}-\check{x}_{k-1}\right)+W w_{k-1} \\
& z_{k} \approx \check{z}_{k}+H\left(x_{k}-\tilde{x}_{k}\right)+V v_{k}
\end{aligned}
$$


Here, $\breve{x}_{k}$ is the a posteriori estimate of the state at step $k, x_{k}$ and $z_{k}$ are the actual state and measurement vectors, and $A, W, H$, and $V$ are the Jacobian matrices calculated by:

$$
\begin{aligned}
& A_{[i, j]}=\frac{d f_{[i]}}{d x_{[j]}}\left(\check{x}_{k-1}, u_{k-1}, 0\right) \\
& W_{[i, j]}=\frac{d f_{[i]}}{d w_{[j]}}\left(\check{x}_{k-1}, u_{k-1}, 0\right) \\
& H_{[i, j]}=\frac{d h_{[i]}}{d x_{[j]}}\left(\tilde{x}_{k}, 0\right) \\
& V_{[i, j]}=\frac{d h_{[i]}}{d v_{[j]}}\left(\tilde{x}_{k}, 0\right)
\end{aligned}
$$

Using this, the predictor-corrector sequence can be formulated as shown in Figure B.1.

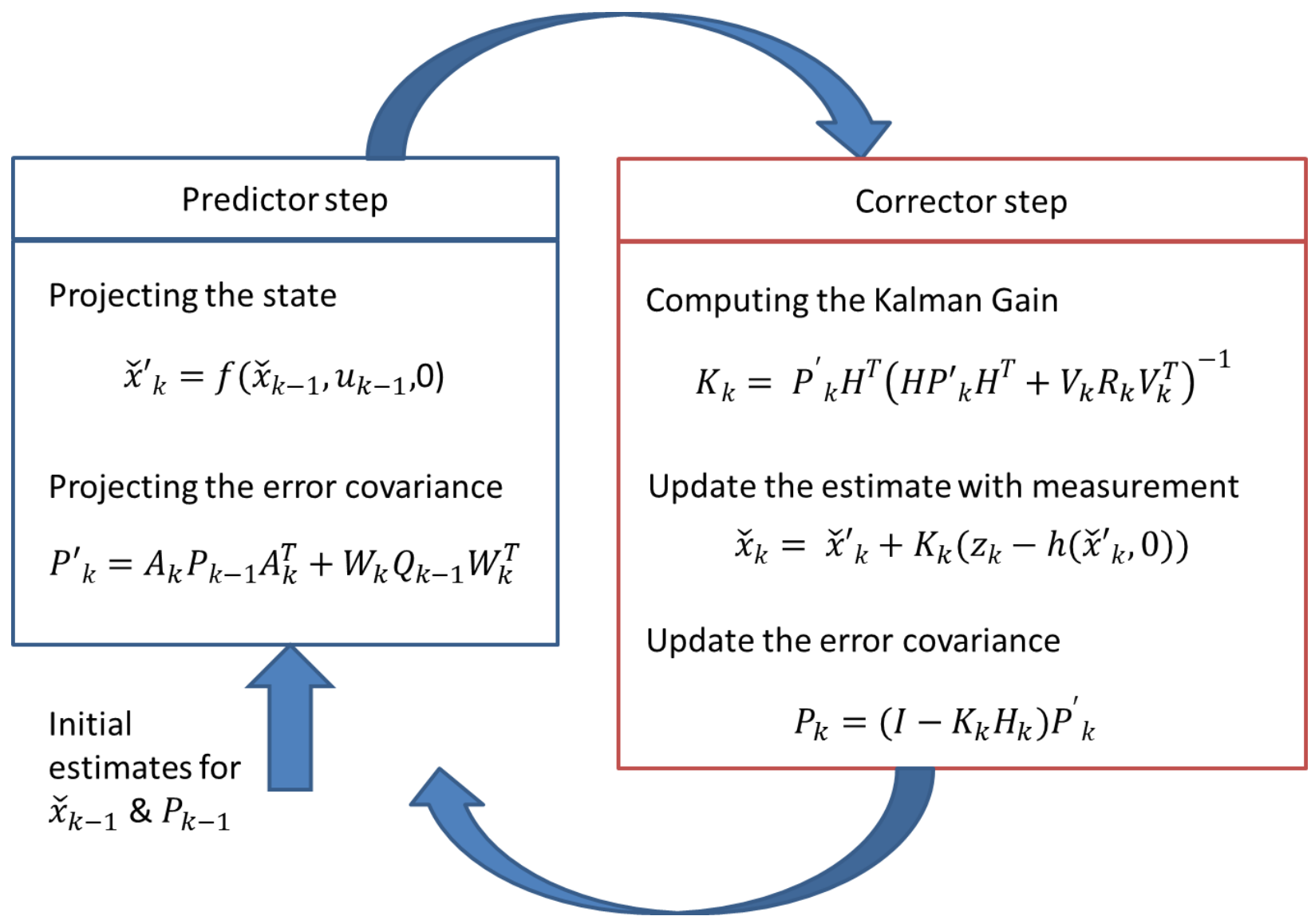

Figure B.1: Flowchart showing the EKF algorithm 
The sensor placement is performed by the GA where the genes are assumed to be measurement models and each measurement model represents a vector of binary decision variables in which if a sensor is placed, the decision variable will take a value of " 1 " and a value of " 0 " otherwise.

\section{The Genetic algorithm}

In the genetic algorithm (GA), the genes represent the binary measurement models that can be used by EKF for state estimation. The fixed population of GA evolves by the elitist selection strategy where a portion of the population is considered as the elite genes and carries over to the next generation. The rest of the population is obtained based on the tournament selection where fitness values of two randomly chosen genes are compared against each other and the gene with highest fitness is considered as the winner. This selection is repeated until two winner genes are selected for crossover and mutation- the GA operators for obtaining the two children from parent genes. In this work the number of measurements (sensors) is fixed for a particular study, the crossover and mutation on the winner genes are repeated until at least one of the children has the same number of fixed measurements. The tournament selection results in obtaining two children per each run, therefore, this process must be repeated until a new population is generated. The objective of the GA is to minimize the error between actual data and the state estimates and the objective function is given by:

$$
\min \sum_{i=2}^{k_{T}} \sum_{j=1}^{n_{T}}\left(\frac{x_{\text {actual },(i, j)}-\hat{x}_{\text {estimated },(i, j)}}{x_{\text {actual },(i, j)}}\right)^{2}
$$

It should be noted that the summation in Eqn. B.25 is over all the estimated values for all time instants while the initial state estimates are discarded and summation start from $i=2$.

For the water gas shift reactor, the parameters used in the EKF are shown in Table B.3. 
Table B.3: EKF parameters for the WGSR model

\begin{tabular}{cc}
\hline EKF parameters & Value \\
\hline Number of grids & 25 \\
Sampling time & $5.4 \mathrm{~s}$ \\
Mole fraction process noise covariance & \\
$\mathrm{CO}, \mathrm{H}_{2} \mathrm{O}, \mathrm{CO}_{2}, \mathrm{H}_{2}$ & $10^{-6}$ \\
$\mathrm{COS}, \mathrm{H}_{2} \mathrm{~S}$ & $2.5 \times 10^{-11}$ \\
& \\
Mole fraction measurement noise covariance & $10^{-6}$ \\
$\mathrm{CO}, \mathrm{H}_{2} \mathrm{O}, \mathrm{CO}_{2}, \mathrm{H}_{2}$ & $10^{-12}$ \\
$\mathrm{COS}, \mathrm{H}_{2} \mathrm{~S}$ & \\
& $2.5 \times 10^{-7}$ \\
Temperature process noise covariance & $10^{-6}$ \\
Temperature measurement noise covariance & $10^{-6}$ \\
Pressure process noise covariance & $2.5 \times 10^{-7}$ \\
Pressure measurement noise covariance & $2.5 \times 10^{-5}$ \\
Fault state process noise covariance & \\
Initial error covariance & $10^{-12}$ \\
COS, $\mathrm{H}_{2} \mathrm{~S}$ & $10^{-6}$ \\
Other states
\end{tabular}




\section{APPENDIX C: DERIVATION FOR ANALYTICAL EQUATIONS FOR STRESS}

The analytical equations for stresses in the radial, hoop and axial direction are derived using the stress-strain equations, strain-displacement relations and the stress equation for radial coordinates.

The stress-strain relation for radial and hoop strains for elastic materials is given as Eqs. C.1-C.2.

$$
\begin{aligned}
& \varepsilon_{r r}=\frac{1}{E}\left[\sigma_{r r}-\vartheta\left(\sigma_{\theta \theta}+\sigma_{z z}\right)\right]+\alpha \Delta T+k_{s l} \\
& \varepsilon_{\theta \theta}=\frac{1}{E}\left[\sigma_{\theta \theta}-\vartheta\left(\sigma_{r r}+\sigma_{z z}\right)\right]+\alpha \Delta T+k_{s l}
\end{aligned}
$$

where, $\sigma_{r r}, \sigma_{\theta \theta}$, and $\sigma_{z z}$ are the radial, hoop and axial stress respectively; $E$ is the Young's modulus, $\vartheta$ is the Poisson's ratio, $\alpha$ is the thermal expansion coefficient and $k_{s l}$ the strain due to the swelling of the slag penetrated refractory. $k_{s l}$ is given as:

$$
k_{s l}=C_{\text {slag }} \frac{\Delta V}{3 V}
$$

Plain strain assumption is made, therefore the axial strain is set to zero, i.e. $\varepsilon_{z z}=0$.

Eqs. C.1 and C.2 can be rearranged in terms of radial and hoop stress to obtain:

$$
\begin{aligned}
& \sigma_{r r}=\frac{E}{(1+\vartheta)(1-2 \vartheta)}\left[(1-\vartheta) \varepsilon_{r}+\vartheta \varepsilon_{\theta}-(1+\vartheta) \alpha \Delta T-(1+\vartheta) k_{s l}\right] \\
& \sigma_{\theta \theta}=\frac{E}{(1+\vartheta)(1-2 \vartheta)}\left[(1-\vartheta) \varepsilon_{\theta}+\vartheta \varepsilon_{r}-(1+\vartheta) \alpha \Delta T-(1+\vartheta) k_{s l}\right]
\end{aligned}
$$

At equilibrium, the stress in the radial direction in cylindrical coordinates is calculated as shown in Eq. C.6.

$$
\frac{d \sigma_{r r}}{d r}+\frac{\sigma_{r r}-\sigma_{\theta \theta}}{r}=0
$$


The strain displacement relations for the radial and hoop direction are given as Eqs. C.7-C.8.

$$
\begin{aligned}
& \varepsilon_{r}=\frac{d u}{d r} \\
& \varepsilon_{\theta}=\frac{u}{r}
\end{aligned}
$$

Substituting Eqs. C.7 and C.8 into Eqs. C.4 and C.5, we get the radial and hoop stress in terms of radial displacement $u$.

$$
\begin{aligned}
& \sigma_{r r}=\frac{E}{(1+\vartheta)(1-2 \vartheta)}\left[(1-\vartheta) \frac{d u}{d r}+\vartheta \frac{u}{r}-(1+\vartheta) \alpha \Delta T-(1+\vartheta) k_{s l}\right] \\
& \sigma_{\theta \theta}=\frac{E}{(1+\vartheta)(1-2 \vartheta)}\left[(1-\vartheta) \frac{u}{r}+\vartheta \frac{d u}{d r}-(1+\vartheta) \alpha \Delta T-(1+\vartheta) k_{s l}\right]
\end{aligned}
$$

Eqs. C.9 and C.10 can be substituted into the radial stress equation C.6. to solve for the radial displacement $u$.

$$
\begin{aligned}
& \frac{d}{d r}\left[(1-\vartheta) \frac{d u}{d r}+\vartheta \frac{u}{r}-(1+\vartheta) \alpha \Delta T-(1+\vartheta) k_{s l}\right]+\frac{1}{r}\left[(1-\vartheta) \frac{d u}{d r}+\vartheta \frac{u}{r}-(1-\vartheta) \frac{u}{r}-\vartheta \frac{d u}{d r}\right] \\
& =0 \\
& \Rightarrow(1-\vartheta) \frac{d^{2} u}{d r^{2}}+\vartheta\left(\frac{r \frac{d u}{d r}-u}{r^{2}}\right)-(1+\vartheta) \alpha \frac{d \Delta T}{d r}-(1+\vartheta) \frac{d k_{s l}}{d r}+\left[\frac{(1-\vartheta)}{r} \frac{d u}{d r}+\frac{\vartheta}{r^{2}} u-(1-\vartheta) \frac{u}{r^{2}}-\right. \\
& \left.\frac{\vartheta}{r} \frac{d u}{d r}\right]=0 \\
& \Rightarrow \frac{d^{2} u}{d r^{2}}+\frac{1}{r} \frac{d u}{d r}-\frac{u}{r^{2}}=\frac{(1+\vartheta)}{(1-\vartheta)}\left[\alpha \frac{d \Delta T}{d r}+\frac{d k_{s l}}{d r}\right] \\
& \Rightarrow \frac{d}{d r}\left[\frac{1}{r} \frac{d(u r)}{d r}\right]=\frac{(1+\vartheta)}{(1-\vartheta)}\left[\alpha \frac{d \Delta T}{d r}+\frac{d k_{s l}}{d r}\right] \\
& \Rightarrow \frac{1}{r} \frac{d(u r)}{d r}=\frac{(1+\vartheta)}{(1-\vartheta)}\left[\alpha \Delta T+k_{s l}\right]+c_{1} \\
& \Rightarrow u=\frac{(1+\vartheta)}{(1-\vartheta)}\left[\frac{\alpha}{r} \int \Delta T r d r+\frac{1}{r} \int k_{s l} r d r\right]+\frac{c_{1} r}{2}+\frac{c_{2}}{r}
\end{aligned}
$$


Substituting $u$ into Eqs. C.7 and C. 8 we obtain the Eqs. C.12 and C.13

$$
\begin{gathered}
\varepsilon_{r}=\frac{(1+\vartheta)}{(1-\vartheta)}\left[\alpha \Delta T-\frac{\alpha}{r^{2}} \int \Delta T r d r+k_{s l}-\frac{1}{r^{2}} \int k_{s l} r d r\right]+\frac{c_{1}}{2}-\frac{c_{2}}{r} \\
\varepsilon_{\theta}=\frac{(1+\vartheta)}{(1-\vartheta)}\left[\frac{\alpha}{r^{2}} \int \Delta T r d r+\frac{1}{r^{2}} \int k_{s l} r d r\right]+\frac{c_{1}}{2}-\frac{c_{2}}{r^{2}}
\end{gathered}
$$

Now, substituting Eqs. C.12 and C.13 into Eq. C.4 to simplifying it, we get Eq. C.14 shown below.

$$
\sigma_{r r}=E\left[-\frac{\alpha}{r^{2}(1-\vartheta)} \int \Delta T r d r-\frac{1}{r^{2}(1-\vartheta)} \int k_{s l} r d r+\frac{c_{1}}{2(1+\vartheta)(1-2 \vartheta)}-\frac{c_{2}}{r^{2}(1+\vartheta)}\right]
$$

$c_{1}$ and $c_{2}$ can be obtained by using the boundary conditions:

$$
\begin{aligned}
& \text { At } \mathrm{r}=\mathrm{a}, \sigma_{r r}=-P_{1} \\
& \text { At } \mathrm{r}=\mathrm{b}, \sigma_{r r}=-P_{2}
\end{aligned}
$$

At $\mathrm{r}=\mathrm{a}$;

$$
\begin{aligned}
-P_{1}=E[- & \frac{\alpha}{a^{2}(1-\vartheta)} \int_{a}^{a} \Delta \operatorname{Tr} d r-\frac{1}{a^{2}(1-\vartheta)} \int_{a}^{a} k_{s l} r d r+\frac{c_{1}}{(1+\vartheta)(1-2 \vartheta)} \\
& \left.-\frac{c_{2}}{a^{2}(1+\vartheta)(1-2 \vartheta)}\right] \\
\Rightarrow c_{1}= & {\left[\frac{-P_{1}}{E}+\frac{c_{2}}{a^{2}(1+\vartheta)}\right] 2 \cdot(1+\vartheta)(1-2 \vartheta) }
\end{aligned}
$$

At $r=b$;

$$
\begin{gathered}
-P_{2}=E\left[-\frac{\alpha}{b^{2}(1-\vartheta)} \int_{a}^{b} \Delta \operatorname{Tr} d r-\frac{1}{b^{2}(1-\vartheta)} \int_{a}^{b} k_{s l} r d r+\left[\frac{-P_{1}}{E}+\frac{c_{2}}{a^{2}(1+\vartheta)}\right]\right. \\
\left.-\frac{c_{2}}{b^{2}(1+\vartheta)(1-2 \vartheta)}\right]
\end{gathered}
$$




$$
\Rightarrow c_{2}=\left(\frac{P_{1}-P_{2}}{E}\right)+\frac{1}{b^{2}} \frac{1}{(1-\vartheta)}\left[\alpha \int_{a}^{b} \Delta \operatorname{Tr} d r+\int_{a}^{b} k_{s l} r d r\right] \frac{(1+\vartheta)}{b^{2}-a^{2}} a^{2} b^{2}
$$

Therefore, substituting $c_{1}$ and $c_{2}$ from Eq. C.17 and C.18 into Eq. C.14 and simplifying, we get:

$$
\begin{gathered}
\sigma_{r r}=\frac{E}{r^{2}(1-v)} \cdot\left(\alpha \int_{a}^{r} \Delta T r d r+\int_{a}^{r} \mathrm{k}_{s l} r d r\right)+\frac{r^{2}-a^{2}}{b^{2}-a^{2}} \frac{E}{r^{2}(1-v)} \cdot\left(\alpha \int_{a}^{b} \Delta T r d r\right. \\
\left.+\int_{a}^{b} \mathrm{k}_{s l} r d r\right)+\frac{P_{1} a^{2}}{b^{2}-a^{2}}\left(1-\frac{b^{2}}{r^{2}}\right)-\frac{P_{2} b^{2}}{b^{2}-a^{2}}\left(1-\frac{a^{2}}{r^{2}}\right)
\end{gathered}
$$

Similarly, the equation for hoop stress, $\sigma_{\theta \theta}$ can be obtained by substituting Eqs. C.12 and C.13 into Eq. C.5 and simplifying, we get Eq. C.20.

$$
\begin{gathered}
\sigma_{\theta \theta}=E\left[\frac{\alpha}{r^{2}(1-\vartheta)} \int_{a}^{r} \Delta \operatorname{Tr} d r+\frac{1}{r^{2}(1-\vartheta)} \int_{a}^{r} k_{s l} r d r-\frac{1}{(1-v)} \alpha \Delta T-\frac{1}{(1-v)} k_{s l}\right. \\
\left.+\frac{c_{1}}{2(1+\vartheta)(1-2 \vartheta)}+\frac{c_{2}}{r^{2}(1+\vartheta)}\right]
\end{gathered}
$$

Using the equations for $c_{1}$ and $c_{2}$ obtained earlier, and simplifying, we get:

$$
\begin{aligned}
\sigma_{\theta \theta}=\frac{E}{r^{2}(1-v)} & \cdot\left(\alpha \int_{a}^{r} \Delta \operatorname{Tr} d r+\int_{a}^{r} \mathrm{k}_{s l} r d r\right) \\
+ & \frac{r^{2}+a^{2}}{b^{2}-a^{2}} \frac{E}{r^{2}(1-v)} \cdot\left(\alpha \int_{a}^{b} \Delta T r d r+\int_{a}^{b} \mathrm{k}_{s l} r d r\right)-E \alpha \Delta T-E . k_{s l} \\
+ & \frac{P_{1} a^{2}}{b^{2}-a^{2}}\left(1+\frac{b^{2}}{r^{2}}\right)-\frac{P_{2} b^{2}}{b^{2}-a^{2}}\left(1+\frac{a^{2}}{r^{2}}\right)
\end{aligned}
$$

Therefore, Eqs. C.15 and C.17 are the analytical equations for the radial and hoop stress respectively. 